\title{
Economic and humanistic impact of medication nonadherence in patients with asthma and chronic obstructive pulmonary disease
}

Ashish V. Joshi

West Virginia University

Follow this and additional works at: https://researchrepository.wvu.edu/etd

\section{Recommended Citation}

Joshi, Ashish V., "Economic and humanistic impact of medication nonadherence in patients with asthma and chronic obstructive pulmonary disease" (2005). Graduate Theses, Dissertations, and Problem Reports. 4157.

https://researchrepository.wvu.edu/etd/4157

This Dissertation is protected by copyright and/or related rights. It has been brought to you by the The Research Repository @ WVU with permission from the rights-holder(s). You are free to use this Dissertation in any way that is permitted by the copyright and related rights legislation that applies to your use. For other uses you must obtain permission from the rights-holder(s) directly, unless additional rights are indicated by a Creative Commons license in the record and/ or on the work itself. This Dissertation has been accepted for inclusion in WVU Graduate Theses, Dissertations, and Problem Reports collection by an authorized administrator of The Research Repository @ WVU.

For more information, please contact researchrepository@mail.wvu.edu. 


\title{
Economic and Humanistic Impact of Medication Nonadherence in Patients with Asthma and Chronic Obstructive Pulmonary Disease
}

\author{
Ashish V. Joshi
}

Dissertation Submitted to the

School of Pharmacy

at West Virginia University

in partial fulfillment of the requirements for the degree of

\author{
Doctor of Philosophy \\ In \\ Pharmaceutical Systems and Policy \\ S. Suresh Madhavan, MBA, Ph.D. \\ Ambarish Ambegaonkar, Ph.D. \\ Virginia (Ginger) Scott, Ph.D. \\ Michael J. Smith, Ph.D., R.Ph. \\ Harakh Dedhia, MD
}

Department of Pharmaceutical Systems and Policy

\section{Morgantown, West Virginia \\ 2005}

Keywords: Medication adherence, healthcare utilization, health-related quality of life, productivity, asthma, and COPD

Copyright 2005 Ashish V. Joshi 


\title{
ABSTRACT \\ Economic and Humanistic Impact of Medication Nonadherence in Patients with Asthma and Chronic Obstructive Pulmonary Disease
}

\begin{abstract}
Ashish V. Joshi
Asthma and chronic obstructive pulmonary disease (COPD) significantly impact morbidity and mortality. In spite of the well-known benefits of prophylactic medication use, especially in asthma, the rate of medication nonadherence is more than $50 \%$. In Phase I, this study examined the relationship between refill-based medication nonadherence and healthcare utilization/costs in patients with asthma, COPD, and those with both asthma and COPD from the West Virginia (WV) Public Employees Insurance Agency (PEIA) program. In Phase II, the study measured the relationship between refillbased and self-reported medication nonadherence, health-related quality of life (HRQL), and losses in workplace productivity, all of which were determined via a mailed questionnaire to patients identified from Phase I. Phase I Results: The prevalence of asthma in the study population was similar to national estimates $(203 / 10,000)$, whereas the prevalence of COPD was higher (598/10,000). Among asthma-only and those with both asthma and COPD, more than half the patients received medications according to NHLBI guidelines. Refill-based medication adherence was highest in patients having both asthma and COPD, as compared to asthma-only or COPD-only enrollees. The number of adverse outcomes such as hospitalizations and ED visits increased with increasing refill-based adherence for the COPD-only patients. Total healthcare costs increased with increasing medication adherence for all three groups. Thus, increasing medication adherence was possibly a reflection of increasing disease severity. Phase II Results: The overall response rate was almost $23 \%(\mathrm{~N}=918)$, and was highest for the asthma-only group (25\%), followed by the 'both' group (24\%), and the COPD-only group (15\%). The perception of HRQL among WV PEIA enrollees was similar to those found in other studies. Only $40 \%$ of all Phase II respondents reported themselves as high adherent; the prevalence of self-reported adherence being similar in all three sub-groups. The correlations between self-reported and refill-based adherence in the three groups were not clinically significant. Medication adherence was a significant predictor of HRQL for the COPD-only group, with HRQL worsening with increasing adherence. Self-reported health status was a significant predictor of HRQL for each of the three disease groups; and HRQL worsened with deteriorating health status. In all three groups, medication adherence was not significantly associated with losses in workplace productivity dollars.
\end{abstract}




\section{ACKNOWLEDGEMENTS}

I would like to take this opportunity to thank all those who have been instrumental in making this study possible. First of all, I would like to sincerely thank my dissertation chairperson and mentor Dr. Suresh Madhavan, for making this possible. His constant support and motivation were always inspiring, even when things were not going according to plan. His constructive criticism was invaluable, and his patience incomparable. Most of all, the completion of my doctoral degree has helped me develop as a person, which is far more important than learning the use of any propensity score analysis or Markov model. I give wholehearted credit to Dr. Madhavan for my entire learning experience, both technically and otherwise.

I would also like to thank Dr. Ambarish Ambegaonkar for his constant support and for being a pseudo-mentor and guide. I would also like to thank Dr. David Day and Pfizer Inc. for their support and for funding this study. I would like to express my sincere gratitude to Dr. Virginia Scott and Dr. Michael Smith for their support and encouragement. No study is possible without clinical input, and I wholeheartedly thank Dr. Harakh Dedhia for his clinical perspective and enthusiasm. A special thanks to West Virginia PEIA for giving us the opportunity to conduct this study, and for being supportive of this study through some challenging times during my research. I would like to thank Shashi for his help with data extraction, and Debra Hardesty and Mickey Howell for their assistance.

Throughout my graduate studies at WVU, I have received terrific support from all the faculty members in the Dept. of Pharmaceutical Systems and Policy. For this, in addition to my committee members, I would also like to thank Dr. Mayur Amonkar, Dr. 
Lesley-Ann Miller, and Dr. Jan Kavookjian for their guidance during various stages of my doctoral studies.

This list would be incomplete without mentioning all my fellow graduate students, who have been extremely cooperative. Mariam Hassan deserves a special mention for being a great friend (and for sometimes helping me with SAS). Murali Sundaram, Rohit Borker, Ancilla Fernandes, Jay Coffindaffer, and Vivek Pawar were as entertaining as always, and definitely helped keep my spirits up.

Finally, I would like to thank my family, who deserve the most credit for all my accomplishments - my parents Vikas and Shashi, and my sister Namita. They have always been my source of strength and inspiration, and none of this would have been possible without their support and encouragement. 


\section{TABLE OF CONTENTS}

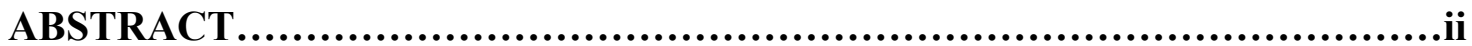

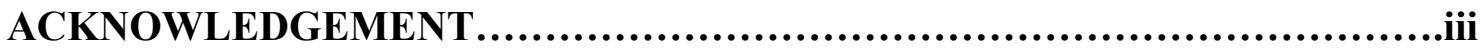

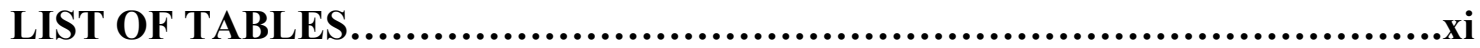

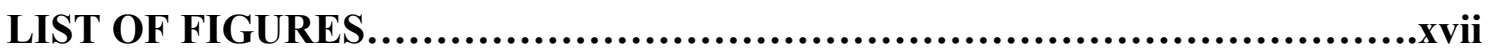

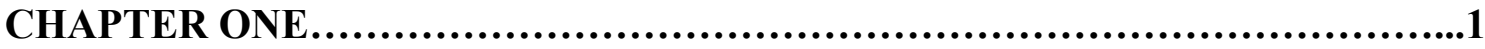

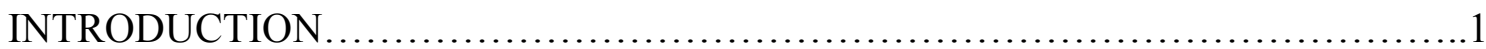

Epidemiology and Burden.....................................................

Pharmacotherapy for Asthma and Chronic Obstructive Pulmonary Disease...........2

Role of Medication Adherence........................................................

Factors Affecting Adherence with Asthma Therapy...............................5

Health-Related Quality of Life in Asthma and COPD...........................6

Nonadherence and Health-Related Quality of Life...............................

Workplace Productivity and Chronic Respiratory Disease..........................8

STATEMENT OF PROBLEM..............................................

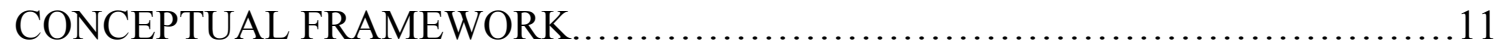

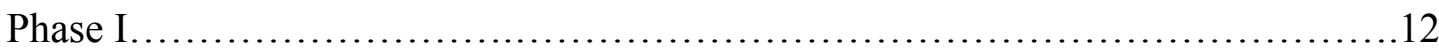

Selection of PEIA Population...............................................12

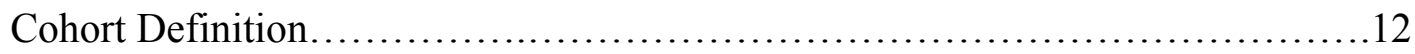

Classification of Patients.................................................... 15

Definition of Medication Adherence.........................................16

Other Independent Variables............................................... 17

Evaluation of Outcomes.................................................... 18 


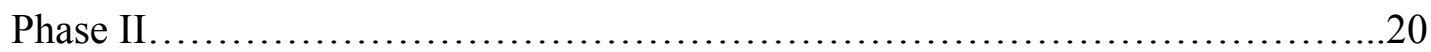

STUDY OBJECTIVES AND RESEARCH QUESTIONS .........................22

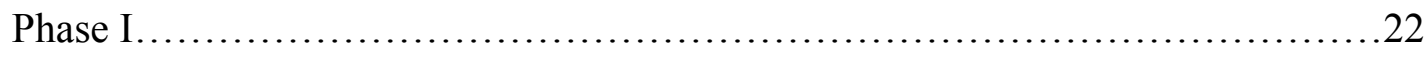

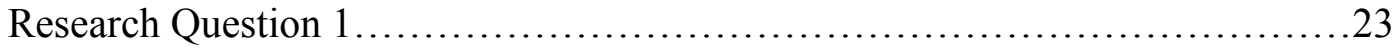

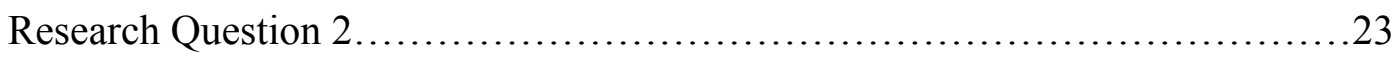

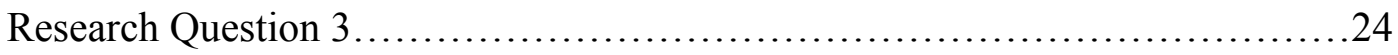

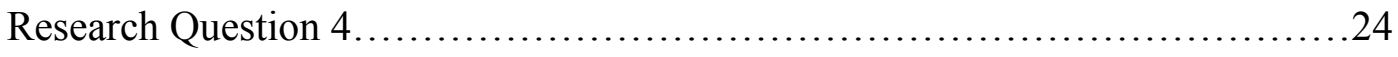

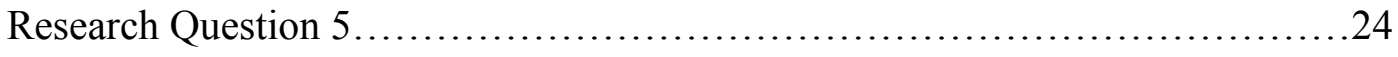

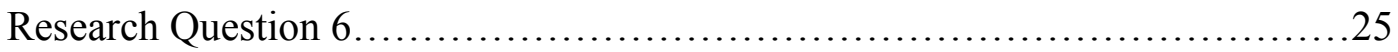

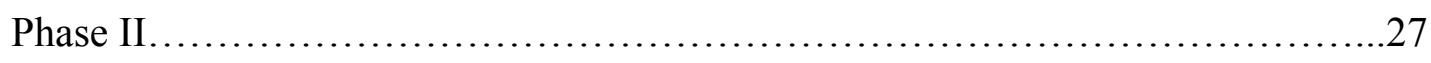

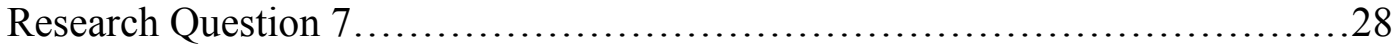

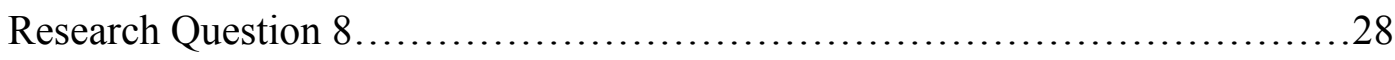

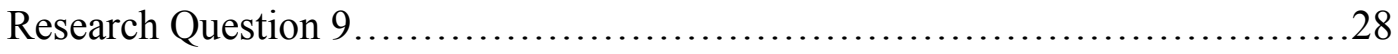

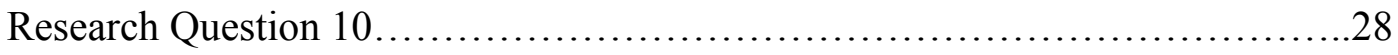

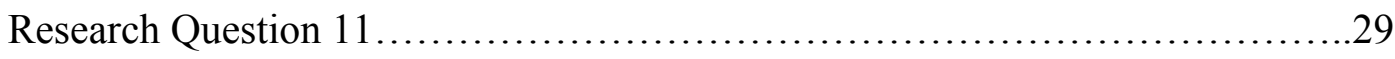

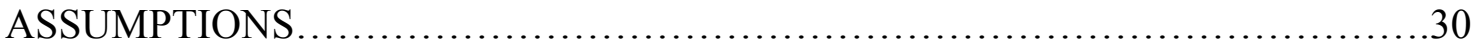

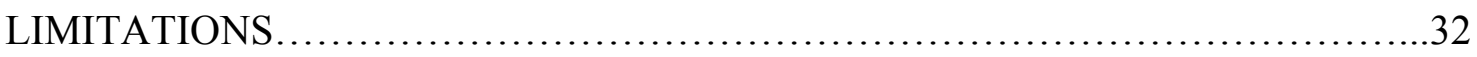

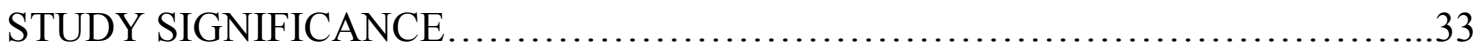

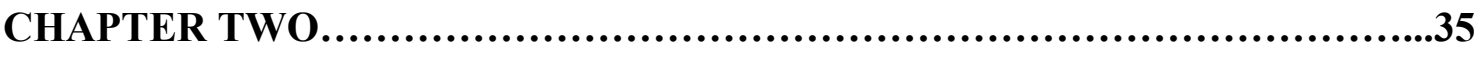

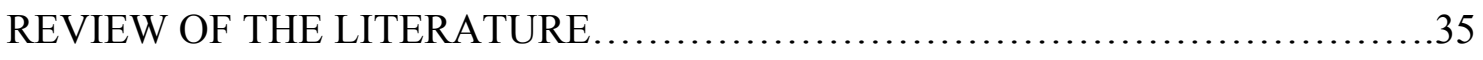

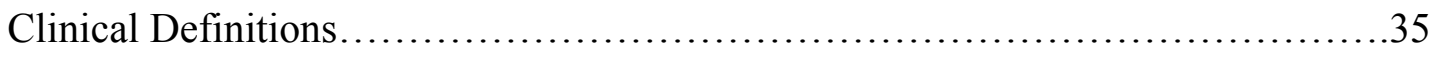

Epidemiology and Burden...................................................40

Prophylactic Therapy in Asthma and Chronic Obstructive Pulmonary Disease.......43 
Clinical Efficacy of Inhaled Corticosteroids: A Review of Meta-Analyses....

Prophylactic Pharmacotherapy and Economic Outcomes......................46

Conceptual Definition of Medication Adherence...............................51

Factors Affecting Medication Adherence...................................52

Measurement of Medication Adherence......................................55

Morisky Medication Adherence Scale......................................57

Workplace Productivity Losses in Asthma and COPD........................57

Measurement of Losses in Workplace Productivity............................58

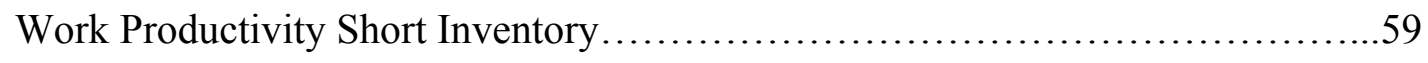

Heath-Related Quality of Life in Asthma and COPD........................6 60

HRQL Instruments Used in Asthma and COPD.............................66

CHAPTER THREE............................................................74

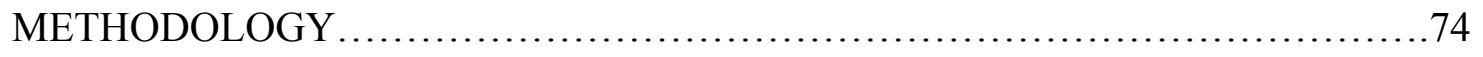

Phase I.............................................................. 74

Data Source..................................................... 74

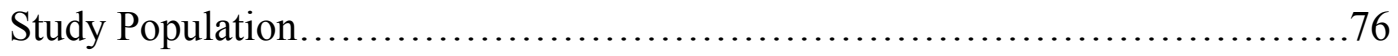

Data Extraction................................................... 76

Data Cleaning and Modification.......................................... 77

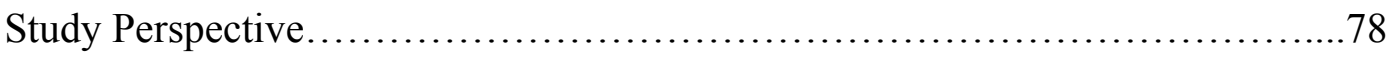

Patient Exclusion, Treatment Arms, and Time Frame....................... 78

Working Definition of Medication Adherence................................ 80

Measurement Indices for Adherence.................................... 80

Demographic Variables................................................ 81 
Measurement of Medication-Related Variables............................81

Index for Measuring Asthma Disease Severity.......................... 82

Research Design..................................................83

Data Analysis........................................................ 83

Phase II............................................................. 97

Study Population..................................................97

Sampling Technique................................................97

Sample Size Determination for COPD-Only Recipients.....................98

Health-Related Quality of Life Assessment..................................99

Self-Reported Nonadherence Assessment.............................. 100

Workplace Productivity Assessment.................................... 101

Data Collection......................................................... 102

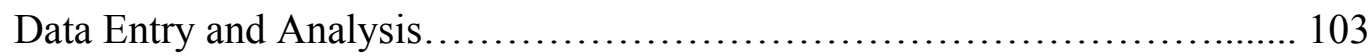

CHAPTER FOUR...................................................................107

RESULTS AND DISCUSSION......................................... 107

Phase I Results.................................................... 107

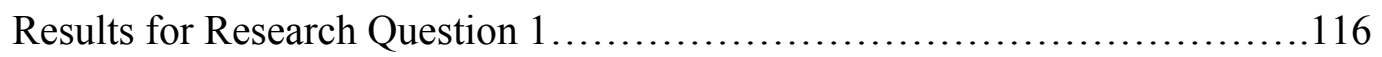

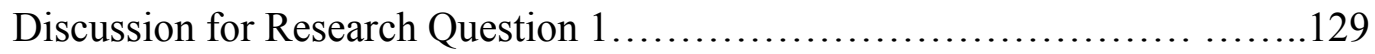

Results for Research Question 2 ....................................130

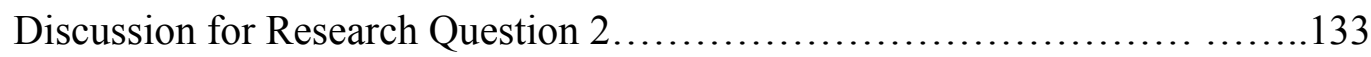

Results for Research Question 3.................................. 134

Discussion for Research Question 3..................................136

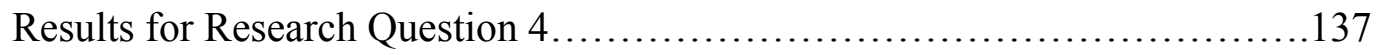


Discussion for Research Question 4...................................166

Results for Research Question 5.......................................168

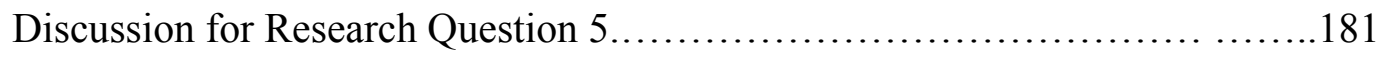

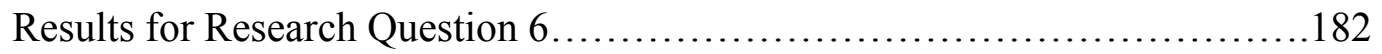

Discussion for Research Question 6....................................189

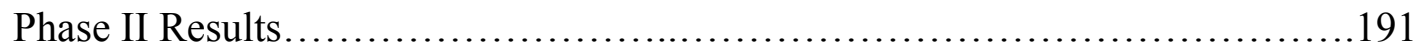

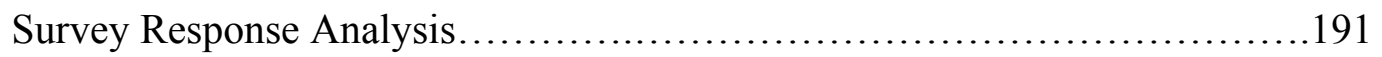

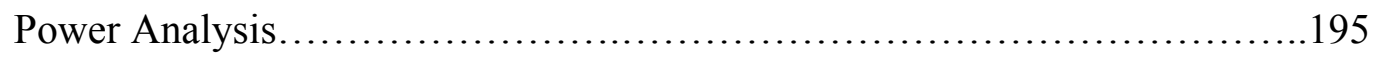

Results for Research Question 7 ...................................... 198

Discussion for Research Question 7 ....................................201

Results for Research Question 8.......................................202

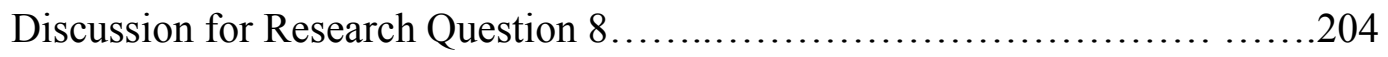

Results for Research Question 9.......................................205

Discussion for Research Question 9...................................211

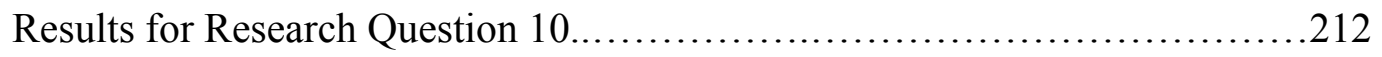

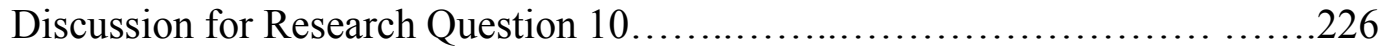

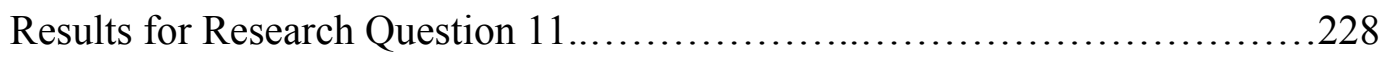

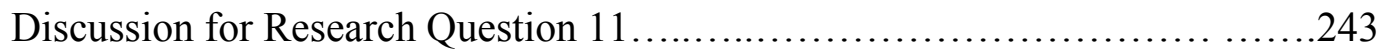

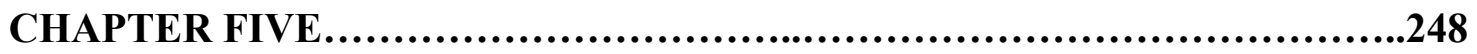

SUMMARY AND CONCLUSIONS.........................................248

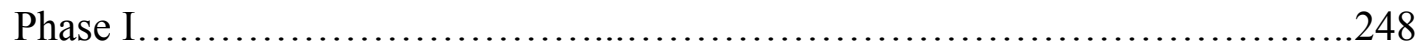

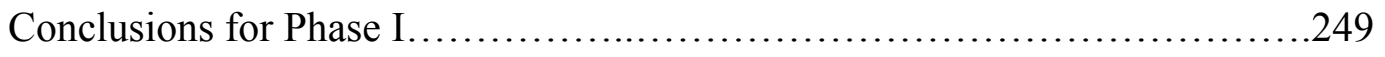

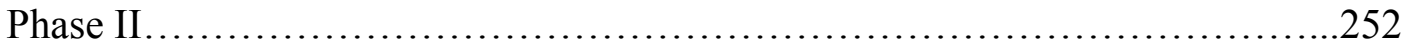


Conclusions for Phase II

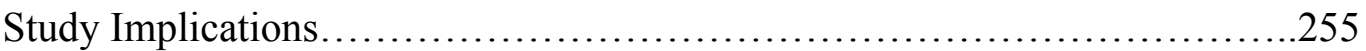

Implications to Health Insurance Programs ...........................255

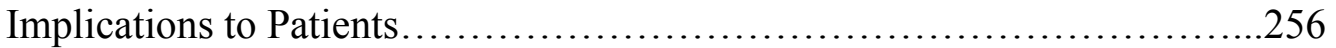

Implications to Prescribers.......................................257

Implications to Decision-Makers..................................257

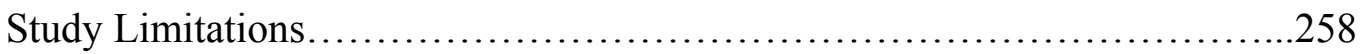

Limitations of Phase I..........................................258

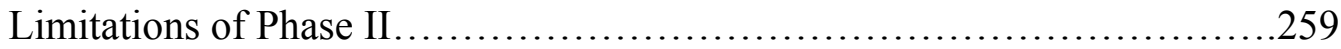

Directions for Future Research..........................................260

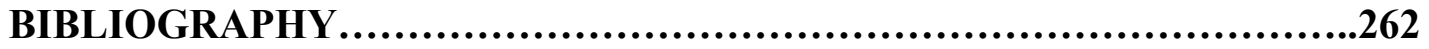

APPENDICES.............................................................277

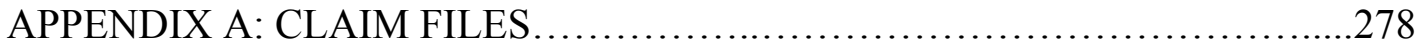

APPENDIX B: CODES FOR IDENTIFICATION ............................280

APPENDIX C: MEDICATIONS .......................................281

APPENDIX D: COVERLETTER.........................................283

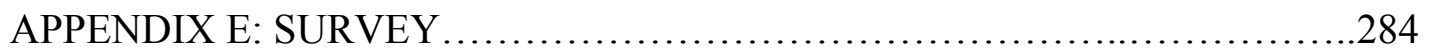

CURRICULUM VITAE....................................................289 


\section{LIST OF TABLES}

Table 1: Clinical Differences between Asthma and COPD.......................38

Table 2: Selection of Study Sample for Phase I...............................109

Table 3: Demographic Characteristics of the Phase 1 Final Study Recipients.....111

Table 4: Expenditures as a Percentage of Total Costs for the Entire Study Period

Table 5: Average Utilization/Costs and Utilization Rates......................115

Table 6: Drug Class by Age Group for Asthma-only Enrollees...................119

Table 7: Drug Class by Gender for Asthma-only Enrollees......................120

Table 8: Drug Class by Age Group for COPD-only Enrollees....................123

Table 9: Drug Class by Gender for COPD-only Enrollees......................124

Table 10: Drug Class by Age Group for Both-Group Enrollees..................127

Table 11: Drug Class by Gender for Both-Group Enrollees......................128

Table 12: Prevalence of Asthma and COPD in WV PEIA Members..............132

Table 13: Distribution of Medication Adherence-Related Variables...............135

Table 14: Poisson Regression Model with Predictors of Number of Adverse

Outcomes using MPR Adherence for the Asthma-Only Group..................139

Table 15: Poisson Regression Model with Predictors of Number of Adverse

Outcomes using Median Gap Adherence for the Asthma-Only Group............140

Table 16: Poisson Regression Model with Predictors of Number of Adverse

Outcomes using MPR Adherence for the COPD-Only Group...................142

Table 17: Poisson Regression Model with Predictors of Number of Adverse

Outcomes using Median Gap Adherence for the COPD-Only Group ............143 
Table 18: Poisson Regression Model with Predictors of Number of Adverse Outcomes using MPR Adherence for the Both Group 145

Table 19: Poisson Regression Model with Predictors of Number of Adverse Outcomes using Median Gap Adherence for the Both Group. 146

Table 20: Poisson Regression Model with Predictors of Number of Adverse

Outcomes using MPR Adherence for the Entire Phase I Sample 148

Table 21: Poisson Regression Model with Predictors of Number of Adverse

Outcomes using Median Gap Adherence for the Entire Phase I Sample

Table 22: Negative Binomial Regression Model with Predictors of Number of

Outpatient Visits using MPR Adherence for the Asthma-Only Group

Table 23: Negative Binomial Regression Model with Predictors of Number of

Outpatient Visits using Median Gap Adherence for the Asthma-Only Group.....153

Table 24: Negative Binomial Regression Model with Predictors of Number of

Outpatient Visits using MPR Adherence for the COPD-Only Group..............155

Table 25: Negative Binomial Regression Model with Predictors of Number of

Outpatient Visits using Median Gap Adherence for the COPD-Only Group......156

Table 26: Negative Binomial Regression Model with Predictors of Number of

Outpatient Visits using MPR Adherence for the Both Group..................158

Table 27: Negative Binomial Regression Model with Predictors of Number of

Outpatient Visits using Median Gap Adherence for the Both Group.............159

Table 28: Negative Binomial Regression Model with Predictors of Number of

Outpatient Visits using MPR Adherence for the Entire Phase I Sample..........161 
Table 29: Negative Binomial Regression Model with Predictors of Number of Outpatient Visits using Median Gap Adherence for the Entire Phase I Sample..162 Table 30: OLS Regression Model with Predictors of LOS using MPR

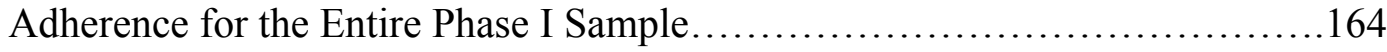

Table 31: OLS Regression Model with Predictors of LOS using Median Gap Adherence for the Entire Phase I Sample....................................165

Table 32: OLS Regression Model with Predictors of Log-Transformed Total Healthcare Costs Using MPR Adherence for the Asthma-Only Group............170 Table 33: OLS Regression Model with Predictors of Log-Transformed Total Healthcare Costs Using Median Gap Adherence for the Asthma-Only Group...171 Table 34: OLS Regression Model with Predictors of Log-Transformed Total Healthcare Costs Using MPR Adherence for the COPD-Only Group...........173

Table 35: OLS Regression Model with Predictors of Log-Transformed Total Healthcare Costs Using Median Gap Adherence for the COPD-Only Group....174 Table 36: OLS Regression Model with Predictors of Log-Transformed Total Healthcare Costs Using MPR Adherence for the Both Group....................176 Table 37: OLS Regression Model with Predictors of Log-Transformed Total Healthcare Costs Using Median Gap Adherence for the Both Group............177 Table 38: OLS Regression Model with Predictors of Log-Transformed Total Healthcare Costs Using MPR Adherence for the Entire Phase I Sample.........179 Table 39: OLS Regression Model with Predictors of Log-Transformed Total Healthcare Costs Using Median Gap Adherence for the Entire Phase I Sample.180 
Table 40: Adjusted Estimates of Odds Ratios Predicting the Risk of Any Adverse Outcome as a Consequence of Adherence Using Logistic Regression Analyses for the Asthma-Only Group. .183

Table 41: Adjusted Estimates of Odds Ratios Predicting the Risk of Any Adverse Outcome as a Consequence of Adherence Using Logistic Regression Analyses for the COPD-Only Group. 185

Table 42: Adjusted Estimates of Odds Ratios Predicting the Risk of Any Adverse Outcome as a Consequence of Adherence Using Logistic Regression Analyses for the Both Group. 186

Table 43: Adjusted Estimates of Odds Ratios Predicting the Risk of Any Adverse Outcome as a Consequence of MPR Adherence Using Logistic Regression Analyses for the Entire Phase I Sample..........................188 Table 44: Number and Percent of Survey Respondents and Non-Respondents

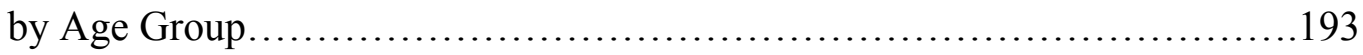

Table 45: Number and Percent of Survey Respondents and Non-Respondents by Gender....................................................... 194

Table 46: Demographic Characteristics of the Phase II Study Subjects........197 Table 47: Health-Related Quality of life Scores for the Phase II Study

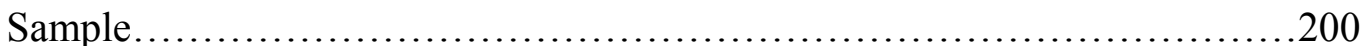

Table 48: Self-Reported Adherence based on the Morisky Scale for the

Phase II Study Sample.............................................203

Table 49: Spearman's Correlation between Self-Reported and Claims-Data Based Adherence for the Entire Phase II Sample...........................206 
Table 50: Spearman's Correlation between Self-Reported and Claims-Data

Based Adherence for the Asthma-Only Group.............................208

Table 51: Spearman's Correlation between Self-Reported and Claims-Data

Based Adherence for the COPD-Only Group..............................209

Table 52: Spearman's Correlation between Self-Reported and Claims-Data

Based Adherence for the Both Group ....................................210

Table 53: OLS Regression Model with Predictors of Total HRQL Score

Using MPR Adherence for the Asthma-Only Group........................214

Table 54: OLS Regression Model with Predictors of Total HRQL Score

Using MPR Adherence for the COPD-Only Group........................216

Table 55: OLS Regression Model with Predictors of Total HRQL Score

Using MPR Adherence for the Both Group..............................218

Table 56: OLS Regression Model with Predictors of Total HRQL Score

Using MPR Adherence for the Entire Phase II Sample.......................220

Table 57: OLS Regression Model with Predictors of Total HRQL Score

Using Morisky Adherence for the Asthma-Only Group......................222

Table 58: OLS Regression Model with Predictors of Total HRQL Score

Using Morisky Adherence for the COPD-Only Group......................223

Table 59: OLS Regression Model with Predictors of Total HRQL Score

Using Morisky Adherence for the Both Group............................224

Table 60: OLS Regression Model with Predictors of Total HRQL Score

Using Morisky Adherence for the Entire Phase II Sample....................225

Table 61: Workplace Productivity Costs for the Phase II Study Sample......229 
Table 62: OLS Regression Model with Predictors of Log-Transformed Total Productivity Dollars Using MPR Adherence for the Asthma-Only Group...231 Table 63: OLS Regression Model with Predictors of Log-Transformed Total Productivity Dollars Using MPR Adherence for the COPD-Only Group.... 233 Table 64: OLS Regression Model with Predictors of Log-Transformed Total Productivity Dollars Using MPR Adherence for the Both Group............235 Table 65: OLS Regression Model with Predictors of Log-Transformed Total Productivity Dollars Using MPR Adherence for the Entire Phase II Sample.237 Table 66: OLS Regression Model with Predictors of Log-Transformed Total Productivity Dollars Using Morisky Adherence for the Asthma-Only Group

Table 67: OLS Regression Model with Predictors of Log-Transformed Total Productivity Dollars Using Morisky Adherence for the COPD-Only Group

Table 68: OLS Regression Model with Predictors of Log-Transformed Total Productivity Dollars Using Morisky Adherence for the Both Group.........241 Table 69: OLS Regression Model with Predictors of Log-Transformed Total Productivity Dollars Using Morisky Adherence for the Entire Phase II Sample 


\section{LIST OF FIGURES}

Figure 1: Conceptual Model Explaining the Relationship between Medication

Adherence and Study Outcomes ....................................... 14

Figure 2: Pharmacotherapy Use among Asthma-Only Enrollees................ 118

Figure 3: Pharmacotherapy Use among COPD-Only Enrollees...................122

Figure 4: Pharmacotherapy Use among Both Group Enrollees..................126

Figure 5: Bivariate Relationship between Productivity Dollars and Age Group...247 


\section{CHAPTER ONE}

\section{INTRODUCTION}

Asthma is a chronic inflammatory disorder, which consists of excessive airway narrowing, in response to a wide range of naturally occurring stimuli (Barbee and Murphy, 1998). More specifically, asthma is characterized by reversibility of airflow obstruction characterized by an inflammatory response of the lungs, either spontaneously or as a result of pharmacotherapy, in addition to bronchial hyperresponsiveness (Bush, 1992).

Chronic Obstructive Pulmonary Disease (COPD) is a disease state characterized by airflow limitation that is not fully reversible (Rennard, 1998). The airflow limitation is usually both progressive and associated with an abnormal inflammatory response of the lungs to noxious particles and gases (Puchelle and Vargaftig, 2001). Individuals diagnosed with any of the following conditions during the course of a lifetime are categorized as suffering from COPD: asthmatic bronchitis, chronic bronchitis, chronic obstructive bronchitis, and emphysema (Pauwels et al, 2001). The clinical definition of each of these is different, although all of the above except simple bronchitis are characterized by airflow limitation.

\section{Epidemiology and Burden}

Respiratory illnesses such as asthma and COPD exert a significant burden on payers, providers, patients, and society. In the United States, asthma was responsible for 500,000 hospitalizations, 1.8 million emergency room visits, 6,500 deaths, 9 million lost work-days, and 10 million lost school days in 1998 (NHLBI, 1999). Asthma was also 
responsible for total cost of 11.3 billion dollars, of which 3.6 billion dollars were due to hospitalizations (NHLBI, 1999). The prevalence of asthma has increased over time, with more than 17.3 million Americans suffering from asthma in 2002, which is a 75 percent increase since 1987 (Mannino et al, 1998). More than 10 percent of children in the United States are affected by asthma, which is one of the most common chronic childhood illnesses (Mannino et al, 1998). Morbidity and mortality due to asthma has increased over the past 2-3 decades, making asthma a major public health concern (Mannino et al, 1998, Evans et al, 1987, Gergen and Weiss, 1990, Weiss et al 1992).

COPD is the fourth leading cause of death in the United States (Hurd 2000, Sullivan et al, 2000). The prevalence of COPD in the United States was estimated to be 61.9/1,000 in 1993 (NHIS, 1993). It is characterized by rising hospitalization rates, along with a concurrent increase in mortality (Sullivan et al, 2000). Annual COPD costs were 14 billion dollars in 2000 (Hurd, 2000). COPD is responsible for 16 million outpatient visits annually (NHLBI, 1998). The age-adjusted death rate for COPD increased by $71 \%$ between 1966 and 1995 (NHLBI, 1998). By the year 2020, COPD is expected to be fifth highest in overall disease burden to society worldwide (Murray and Lopez, 1996).

\section{Pharmacotherapy for Asthma and Chronic Obstructive Pulmonary Disease}

Treatment for asthma and COPD consist of pharmacological therapy, acute care/hospitalization, lifestyle modifications, as well as the use of invasive procedures (NHLBI, 1997 and Pauwels et al, 2001). Pharmacologic therapy for asthma consists of long-term-control medications used to achieve and maintain control of persistent asthma, and short-term medications used to manage acute episodes of asthma exacerbation 
(NHLBI, 1997). Long-term-control medications include: inhaled corticosteroids (ICS), cromolyn sodium and nedocromil, long-acting $\beta_{2}$-agonists, methylxanthines, and leucotrine modifiers. Short-acting $\beta_{2}$-agonists, anticholinergics and systemic corticosteroids are used to manage acute exacerbations. It has been shown that mean forced expiratory volume in one second (FEV1) improved by 25\% among patients who started ICS within six months of the onset of asthma symptoms, as compared to $8 \%$ for patients who were symptomatic 2-5 years before initiating ICS treatment (Selroos et al, 1995). Pharmacologic treatment for COPD consists of bronchodilators (long-acting and short-acting $\beta_{2}$-agonists, anticholinergics, methylxanthines), and glucocorticosteroids (inhaled as well as oral) (Pauwels et al, 2001).

\section{Role of Medication Adherence}

Medication adherence is defined as the "extent to which a person's medicationtaking behavior coincides with medical advice.” (Haynes et al, 1979) Full medication adherence occurs when all the instructions of the prescriber are followed. Fish and Lung (2001) have defined nonadherence as consisting of any of the following behaviors: failure to fill a prescription, consuming the medication at an incorrect dosing interval, consuming incorrect / suboptimal doses of the medication, premature discontinuation of medication, use of an incorrect administration technique, taking the medication for the wrong disease condition, and substitution of one drug for another.

Lack of compliance with prescribed drug regimens costs the United States $\$ 100$ million annually (National Pharmaceutical Council, 1992). Plus, there exists a circuitous relationship between adherence and outcomes, where nonadherence to medications 
impacts clinical severity, which in turn results in adverse outcomes (Fish and Lung, 2001). It has been shown that emergency visits due to asthma exacerbations costs more than $\$ 1.6$ billion annually, and a part of these expenditures can be attributed to nonadherence (Weiss et al, 1992).

Generally, adherence rates for asthma medications range between 3 to $46 \%$, with the mean being less than 50\% (Fish and Lung, 2001, Kelloway et al, 1994, Barr et al, 2002, Bauman et al, 2002, Bender, 2002). This accounts for a significant proportion of high costs, both to the person as well as the society. Among children too, the adherence rates have been found to be lower than 50\% (Bender, 2002). Various investigators have tried to assess the prevalence of nonadherence among patients with asthma. Watts and colleagues (1997) matched asthma prescriptions written by the physician with those actually dispensed over a 3-month period in a rural setting in Australia. They observed that only $70 \%$ of the prescriptions were dispensed by the pharmacies, which results in a primary noncompliance rate of $30 \%$. The actual nonadherence rate may be even higher considering that the actual adherence rate after receiving the prescription is less than $50 \%$. Some studies have tried to assess the extent of nonadherence with inhaler therapy, since ICS form the mainstay of asthma treatment. Apter and colleagues (1998) found that mean adherence to twice daily ICS was $63 \%$, with only $54 \%$ of their patients recording at least $70 \%$ of the prescribed number of doses.

Nonadherence to asthma medications significantly impacts economic as well as clinical outcomes, although there is relatively less evidence of its impact on humanistic outcomes such as health-related quality of life (HRQL). Nonadherence significantly increases the rate of acute exacerbations, which consequently increase emergency 
department (ED) use and hospitalizations, contributing to spiraling healthcare costs. Bradley and colleagues (1999) have estimated that noncompliance to asthma medications contributes to treatment failure in $50 \%$ of the patients with asthma. Braunstein et al (1996) have shown that noncompliance with asthma therapy increased severity and frequency of asthma exacerbations, combined with hospitalizations, resulting in increased morbidity.

\section{Factors Affecting Adherence with Asthma Therapy}

The factors that impact adherence / nonadherence can be grouped into three broad categories such as: (1) treatment-related factors, (2) patient-related factors, and (3) healthcare professional-related factors. Treatment-related factors consist of regimen complexity (Tashkin 1995, Greenberg 1984), mode of administration (Ringdal et al, 1998, Osman, 1996, Fish and Lung, 2001), cost, and prolonged therapy. Patient-related factors consist of knowledge of the disease, disease severity (Leidy, 1995), and selfefficacy. Healthcare professional-related factors consist of good communication with the physician, and regular access to care.

Various studies have examined the factors that affect nonadherence to asthma therapy. Leickly and colleagues (1998) examined the relationship between self-reported adherence, asthma management behavior, and barriers to care among inner city children visiting an ED. The study measured adherence in terms of four aspects: medicine use, appointment-keeping, emergency actions and asthma attack prevention. Side-effects were significantly higher among nonadherent patients, as were doubts regarding the usefulness of medications. Overall, the study showed that barriers to adherence may 
exist in any of these four areas, which may adversely impact asthma control. A study by Barr and colleagues (2002) examined adherence to asthma therapy among older women and identified predictors of adherence. The researchers found that adherence with guidelines decreased as severity increased. Severity, increasing age, lower socioeconomic status (SES), smoking, and a number of medical conditions were significantly associated with asthma, whereas measures of social isolation, emotional well-being, and caregiving were not.

\section{Health-Related Quality of Life in Asthma and COPD}

HRQL has been defined as "the functional effects of illness and its consequent therapy upon a patient, as perceived by the patient.” (Schipper et al, 1996). Overall, research in asthma and COPD has focused on the economic impact of asthma and COPD, with relatively little research measuring the humanistic impact of these diseases. This gap needs to be addressed since both asthma and COPD have a considerable impact on physical, psychological and social domains of HRQL. Some studies have found small relationships between HRQL and pulmonary function in patients with asthma. Bousquet and colleagues (1994) found correlations ranging from 0.35 to 0.47 for various domains of the SF-36 such as physical functioning, physical role functioning, general health perception, and pain, with FEV1 levels. Disease-specific HRQL measures have also been shown to correlate poorly with clinical endpoints in asthma and COPD (van Molken et al, 1997, Juniper et al, 1995, Leidy and Coughlin, 1997, van Molken et al, 1995). Strong correlations have been shown between pulmonary function and HRQL in the symptoms domain of Juniper's Asthma Quality of Life Questionnaire (J-AQLQ) 
(Juniper et al, 1995, Leidy and Coughlin, 1997). Among instruments that can be used in both asthma as well as COPD, the St George's Respiratory Questionnaire (SGRQ) (Jones et al, 1992) is the most stable instrument.

\section{Nonadherence and Health-Related Quality of Life}

An important aspect of asthma therapy is to improve humanistic outcomes such as HRQL, in addition to the more tangible clinical and economic outcomes. Although the bulk of the health outcomes research in asthma has focused on the impact of medication use and economic outcomes such as healthcare use and costs, or the impact of medication nonadherence on economic outcomes, surprisingly, very little research has examined the relationship between nonadherence and HRQL. Berzon and colleagues (1995) have suggested that adherence can be impacted by a medication's side-effect profile, which can subsequently impact HRQL. Thus, any direct evidence of nonadherence on HRQL in chronic pulmonary disease is still lacking.

Creators of various asthma-specific HRQL instruments have always included factors related to medication use in order to explain changes in HRQL. These include bothersome aspects of symptoms, side-effects, and inconvenience due to medication regimen. For instance, the J-AQLQ includes questions pertaining to symptoms, medication costs, and medication availability. Similarly, the Living with Asthma Questionnaire (LWAQ) (Hyland, 1991), and the SGRQ (Jones et al, 1992) ask questions pertaining to medication side-effects, nuisance, access, and effectiveness. These are all factors that impact adherence but do not directly quantify adherence. Overall, there is a dearth of evidence pertaining to the relationship between nonadherence and HRQL. Since 
HRQL is a subjective outcome, it may not be very easy to examine this relationship. For instance, a patient who is worried about the disease, may adhere to the complex treatment regimen, but may still experience lower HRQL, due to a feeling of poor well-being. The vice-versa can also be true, where patients who are suffering from depression either as a function of asthma, or other factors, may be nonadherent to the regimen, thereby aggravating symptoms and exacerbations (Bosley et al, 1995). These patients are more likely to report poorer HRQL.

\section{Workplace Productivity and Chronic Respiratory Disease}

There has been a growing interest in measuring the impact of chronic diseases on workplace productivity in the more recent past (Kessler et al, 2001). However, there is not enough evidence regarding the impact of asthma and COPD on workplace productivity. This is in spite of the fact that tremendous productivity losses have been attributed to asthma (Wiess et al, 1992). Chronic disease like asthma and COPD would not only result in lost workdays (absenteeism), but also reduce productivity while at work (presenteeism). Only few studies have examined the impact of specific anti-asthma drugs on workplace productivity either. One study examined the impact of inhaled terbutaline, an inhaled beta-agonist, on productivity in school children and adults (Northfield et al, 1991). Adults and children who used diaries to record either lost workdays or schooldays, respectively, experienced a decrease in the lost workdays or school-days by $57 \%$ in an open label, single group trial of patients with asthma in primary care. Another study reported losses in workdays due to asthma (Kessler et al, 2001). The study showed that on average, patients with asthma experienced a loss of 3 workdays in a calender year. It 
has been reported that patients with emphysema (COPD) experienced around 19 mean absenteeism days, and losses in presenteeism equivalent to almost 28 days per year (Wang et al, 2003). The same study also reported significant losses in absenteeism (11 days per year) and presenteeism (18 days per year) in patients with asthma.

\section{STATEMENT OF PROBLEM}

In spite of the well-known benefits of asthma medications (NHLBI Guidelines, 1997, Selroos et al, 1995, Olivieri et al, 1997, Balkrishnan and Christensen, 2000), adherence to the medication regimen continues to be a major problem among patients with asthma. The adherence rates among patients with asthma range from 3-46\% (Fish and Lung, 2001, Bender, 2002, Bauman et al, 2002). On average, more than half of the adult patients with asthma continue to remain non-adherent (Fish and Lung, 2001). The rate of nonadherence among children is also similar (Fish and Lung, 2001). Conceptually, one would expect nonadherence to impact both economic as well as humanistic outcomes in patients with chronic pulmonary disease. First, patient, physician, as well as disease-related factors would impact nonadherence. Nonadherence would subsequently impact healthcare utilization and costs, as well as HRQL. HRQL can independently impact healthcare utilization, albeit HRQL itself would also be a function of perceived control and to a certain extent, disease severity.

The relationship between nonadherence, healthcare utilization and costs, and HRQL has not been examined in a single study, although studies have assessed the relationship between nonadherence and its impact on healthcare utilization (Olivieri et al 1997, Balkrishnan and Christensen, 2000), and HRQL and its impact on future healthcare 
use and costs (Eisner et al, 2002). Appropriate use of ICS has been associated with a significant decrease in acute exacerbations, hospital and emergency room use, and mortality in asthma patients (Olivieri et al, 1997, Balkrishnan and Christensen, 2000). Eisner and colleagues (2002) have also shown that better baseline HRQL scores were associated with significantly lower odds of future hospitalization, and significantly lower total healthcare costs in asthma patients, after controlling for sociodemographic as well as clinical factors. Another study has shown that a composite measure of patients' selfreported health status (encompassing presence of comorbidities, quality of life and functioning, prior health service use, and medication use) to be highly correlated with healthcare utilization and costs (Balkrishnan et al, 2000). Vollmer and colleagues have also shown that patients' perception of asthma control significantly impact healthcare utilization, as well as HRQL (Vollmer et al, 1999). They found that outpatient visits, hospital use, and emergency room use increased, as the problems with asthma control increased. Thus, not only nonadherence, but also patients' perception of control, which encompasses patient management, medication adherence, as well as disease severity significantly impact asthma outcomes.

Adherence to maintenance therapy has been shown to improve outcomes in patients with chronic asthma. The regular use of ICS has also been recommended as the mainstay of asthma therapy by the National Heart, Lung, and Blood Institute (NHLBI) Expert Panel 2 guidelines. In COPD, maintenance therapy has not contained the progression of the disease, but it has reduced the rate of acute exacerbations and mortality, and improved self-perceived health status and HRQL. However, the 
effectiveness of these medications is a function of adherence, which continues to be a chronic problem, especially among patients with asthma.

The primary objective of this study is to address this gap by examining the impact of nonadherence to pharmacotherapy on healthcare utilization and costs, and HRQL in the West Virginia (WV) Public Employees Insurance Agency (PEIA) population. PEIA provides healthcare insurance coverage to state employees. These benefits are provided to PEIA employees in terms of reimbursement for the use of prescription drug services, outpatient use, hospital use, emergency room use as well as prescription use. This study will measure medication refill adherence from pharmacy claims data, using indices previously developed and validated. Nonadherence and economic outcomes such as healthcare utilization and costs would be measured using PEIA administrative claims data. HRQL would be measured using a previously validated instrument. The secondary analysis would constitute Phase 1 of the study, and the primary component would constitute Phase 2.

\section{Conceptual Framework}

The purpose of this research is to examine the relationship between medication nonadherence, healthcare utilization and costs, and HRQL in asthma and COPD patients. This study consists of two phases. Phase I consists of examining the relationship between medication nonadherence, and healthcare utilization and costs in patients with asthma and COPD. Phase II consists of examining the relationship between nonadherence to medication regimen and its impact on HRQL in patients with asthma and COPD. 


\section{Phase I}

The following sections will describe the rationale for the selection of WV PEIA population for this study, cohort definition, selection of independent variables, medication adherence, and evaluation of patient outcomes in this study.

\section{Selection of PEIA Population}

This study will examine the impact of medication adherence on economic outcomes such as healthcare utilization and costs in Phase I in WV PEIA covered members with asthma and COPD. PEIA provides healthcare insurance coverage to its state employees for inpatient and outpatient services, prescription drugs, laboratory tests, home healthcare, nursing home care, family planning, and supplies. Thus, a retrospective analysis of PEIA claims data can provide all the required data in one place. Also, the impact of medication adherence on outcomes has not been examined previously in the PEIA population. In asthma, there are specific guidelines recommending the use of maintenance medications, such as ICS (NHLBI, 1997).

\section{Cohort Definition}

The WV PEIA program maintains pharmacy and medical claims data for its recipients from providers of healthcare services. This data includes the date when the service was provided, type of service, amount paid, type of provider, and recipient number. Information about utilization and expenditures for services can be extracted for 
various time periods from the claims data. For this study, all PEIA recipients with a primary 
Figure 1: Conceptual Model Explaining the Relationship between Medication Adherence and Study Outcomes

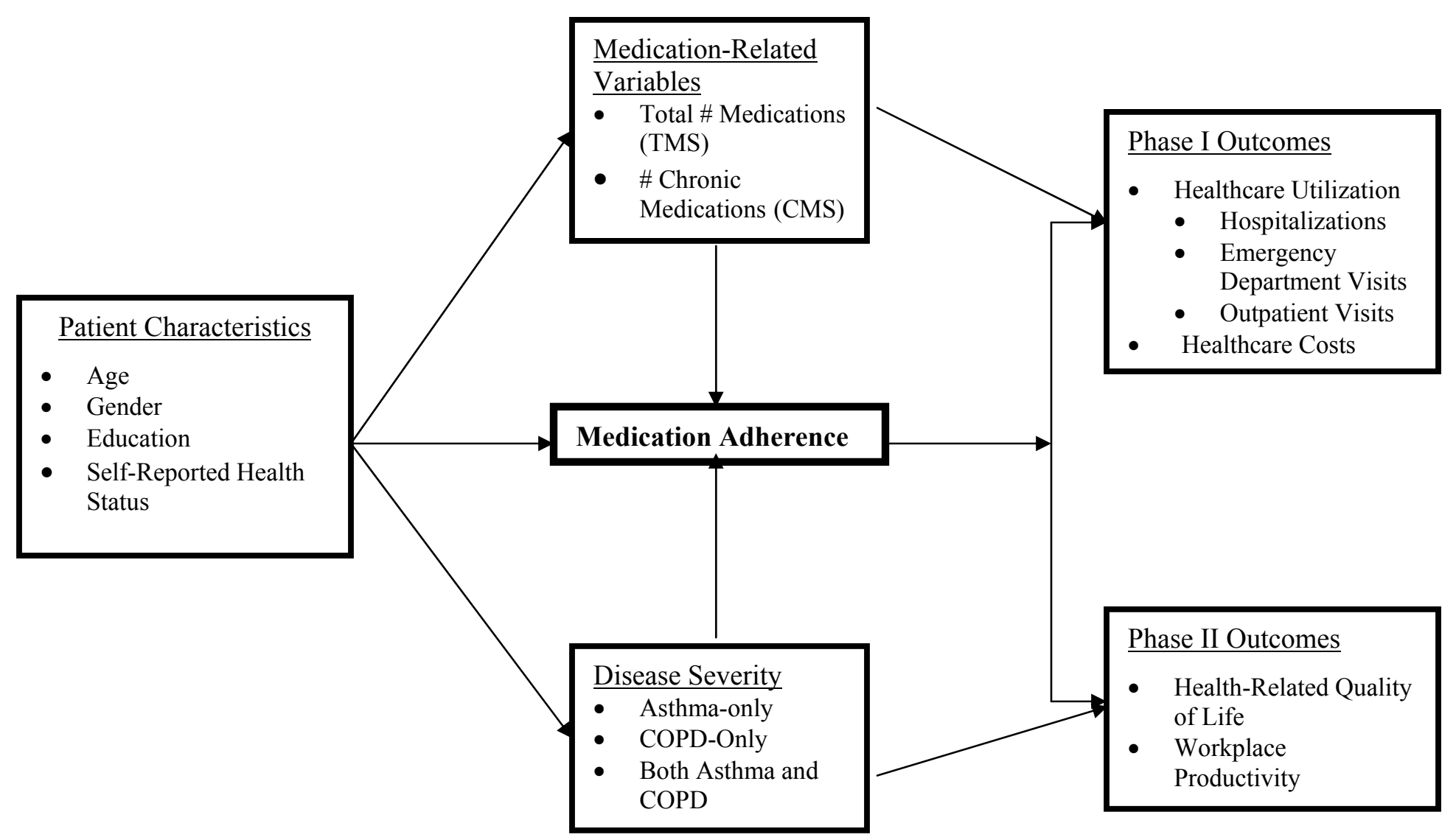


diagnosis code for asthma or COPD between July 2001 and June 2003 in their medical claims will be used. Pharmacy claims for these recipients will be extracted for this time period. In addition, medical and pharmacy claims data for these recipients from July 2003 to December 2003 will also be extracted. From these patients, all members less than 18 years of age and greater than 64 years will be removed. Enrollees more than 64 years of age will not be included since they have Medicare as the primary insurer and data would be incomplete in PEIA files. For the same reason, managed care enrollees will also be excluded. Also, since the study includes sending a mail survey in Phase II, members less than 18 years of age were excluded in order to address informed consent and confidentiality issues.

\section{Classification of Patients}

Based on their International Classification of Diseases, $9^{\text {th }}$ Edition, Clinical Modification (ICD9) codes, the patients will be classified into 3 groups - asthma-only, COPD-only, and recipients having both asthma and COPD. This classification will also be used as a proxy for chronic respiratory disease severity. The assumption is that patients with both asthma and COPD are most severe, followed by COPD-only, and asthma-only patients, in that order. In addition, for the asthma-only group, patients will be categorized into 5 disease severity levels based on a pharmaceutically determined asthma-specific disease severity index (Grana et al, 1997). This index categorizes patients from Level I through Level V - Level I being least severe, and Level V being most severe. Since claims data do not have information on clinical measures of disease 
severity, this classification will serve as a proxy for disease severity for the purpose of this study.

\section{Definition of Medication Adherence}

Medication adherence has been defined as the "extent to which a person's medication-taking behavior coincides with medical advice.” (Haynes et al, 1997) Medications for asthma and COPD have been categorized into two main classes - longterm control medications or maintenance medications to be used prophylactically, and medications for the treatment of acute exacerbations. The first category includes medications such as ICS, long-acting beta agonists, and leucotrine modifiers, whereas the second category includes anticholinergics, short-acting beta agonists, and oral corticosteroids. For Phase I, refill frequency for the maintenance medications will be used to measure adherence. Refill-based indices have been shown to be valid measures of medication adherence (Steiner et al, 1988). Two indices - the medication possession ratio (MPR), and median gap will be used to measure adherence. Although both indices use refill frequency to measure adherence, MPR is defined as the sum of days supply for all claims during a defined period of time divided by the number of days elapsed during the time period. For this study, the MPR will be based on a 365-day time period (Motheral and Fairman, 2000). The second index - median gap - is defined as the number of days between the assumed date of depletion of one claim, and the fill date of the next refill (Motheral and Fairman, 2000). Refill-based adherence will be used as a primary independent variable in Phase I. 
Prescription refill rates provide valuable information regarding the frequency and timeliness of refills of prescribed medications (Farris et al, 1994, Christensen et al, 1997). In the presence of an integrated pharmacy system, monitoring refill patterns can be an effective way of assessing adherence. The other advantage of using refill patterns is that patients are not aware that adherence is being measured, which tends to eliminate any potential biases arising out of a testing effect (Motheral and Fairman, 2000). There are however, certain limitations to this approach as well. It can be hard to measure adherence in patients having access to multiple pharmacies, and to assess unusual refill patterns and multiple conflicting drugs. The biggest limitation is the assumption that "a prescription filled is a prescription taken." (Motheral and Fairman, 2000)

\section{Other Independent Variables}

The other independent variables used in Phase I include the total number of medications (TMS) used, as well as the total number of chronic medications (CMS) used. TMS is defined as the total number of medications for which the patient has had claims throughout the year, and CMS is defined as the total number of medications for which the patient has 4 or more refills per year (Balkrishnan and Christensen, 2001). The use of these measures serves two purposes. First, both TMS and CMS would impact the patients' adherence behavior. Conceptually, patients having a high TMS and/or CMS would indicate a complicated disease regimen thereby potentially impacting adherence to asthma and COPD-related medications. Second, TMS and CMS also serve as proxies for overall patient health status, a high TMS/CMS would indicate deteriorating overall health 
status, which could potentially impact adherence to asthma and COPD medications as well.

\section{Evaluation of Outcomes}

Maintenance medications for asthma when taken regularly show significant benefits in terms of the reduction of adverse outcomes such as hospitalizations and ED visits, as well as mortality. Some benefits have been shown in COPD as well (Rowe et al, 1992 and Hatoum et al, 1994), however, the evidence in the literature has not been convincing enough to recommend maintenance medications in formal guidelines. The cohort of patients selected in this study will be followed to detect changes in healthcare utilization and costs, as well as the risk of adverse outcomes. The study will first examine whether physician prescribing is in accordance with guidelines - NHLBI guidelines for asthma, and GOLD guidelines for COPD (Pauwels et al, 2001). The proportion of patients with asthma receiving ICS would indicate the extent to which prescribing is in accordance with guidelines. At the same time, a high percentage of respondents receiving short-acting beta agonists would indicate the lack of prophylactic medication use among these patients, leading to acute exacerbations, which have to be treated with short-acting beta-agonists. For COPD, since guidelines do not explicitly recommend the use of any particular agent, the proportion of COPD patients receiving different classes of medications will be examined. The prevalence of chronic respiratory illness in WV PEIA members will also be measured. Prevalence rates will be compared between age groups, and gender. 
Since medication nonadherence would result in adverse outcomes such as hospitalizations and ED visits, as well as result in outpatient visits, the impact of medication adherence on the utilization variables will be measured using regression analyses. Regression analyses will be conducted for each of the utilization variables with medication adherence as the primary predictor variable. The use of regression will allow us to measure the impact of adherence after adjusting for various covariates such as age, gender, TMS, CMS, as well as proxies for disease severity. Regression analyses will also be used to examine the impact of medication adherence on total asthma and COPD related healthcare costs. Since a small number of patients are responsible for the major proportion of total costs (skewed data), logarithmic transformations will be used to achieve normality.

The relationship between medication adherence and outcomes should be measured prospectively as well. The impact of medication adherence in a temporal manner will enable the measurement of adverse outcomes such as hospitalizations and ED episodes as a consequence of adherence. A case-control study design will be employed, where patients having adverse outcomes (cases) will be compared with those that do not (controls). For cases, the refill patterns prior to the adverse outcomes will be examined and for controls the refill patterns will be examined for a predefined period. Results from this analysis will allow the estimation of a relative risk estimate - the probability of experiencing an adverse outcome among patients who are adherent versus those who are not. 


\section{Phase II}

The main objective of Phase II of the study is to determine the impact of medication adherence on HRQL in PEIA enrollees with asthma and COPD. The other objectives of Phase II includes the measurement of self-reported medication adherence, and the estimation of losses in workplace productivity in terms of work-days lost due to days absent from work (and corresponding absenteeism dollars), and number of hours unproductive while at work (and corresponding presenteeism dollars) due to asthma and COPD. This phase will involve sending a survey to PEIA members suffering from asthma and COPD who were identified in Phase I.

PEIA members with asthma and COPD will be sent a survey consisting of the following components: a disease-specific HRQL instrument, a self-report adherence scale, and the Work Productivity Short Inventory (WPSI) - a scale used to measure losses in workdays and unproductive hours while at work due to asthma and COPD. In addition, questions about age, gender, education and self-reported health status will also be included. Unfortunately, although there is considerable evidence of the impact of medication adherence and economic outcomes, there is very scant literature examining the relationship between medication nonadherence and HRQL. Conceptually, HRQL should improve with increasing adherence to maintenance medications, however, since HRQL is patients' perception of health, it can be very subjective and does not always correlate well with clinical measures of disease severity. The relationship between medication adherence is very complex. On one hand, improved medication adherence should result in fewer adverse outcomes and consequently improve HRQL. However, it is also possible that increase in disease severity would result in poor HRQL, consequently 
impacting medication adherence. Thus, the study explores the relationship between HRQL in conjunction with medication adherence, and healthcare utilization and costs.

The survey used to measure HRQL is the SGRQ (Jones et al, 1992). This questionnaire has been created and validated for use in patients suffering from both asthma as well as COPD. This questionnaire consists of three domains - symptoms, activity, and impacts. In addition, a total HRQL score is also available. The instrument also defines a clinically significant change in HRQL as 4 units, which is particularly useful since differences between groups may not be statistically significant but clinically meaningful. HRQL scores can thus be compared between asthma and COPD members, and members suffering from both asthma and COPD. The impact of medication adherence on HRQL will be measured using regression analyses, which will allow for the adjustment for various covariates such as age, gender, TMS, CMS, and disease severity.

Medication adherence was measured in phase I using refill-based measures. Since refill-based measures have their limitations, a self-report measure of medication adherence was also incorporated into the survey. This measure, known as the Morisky scale is a generic, 4-item measure of medication adherence, which classified patients as high, medium or low adherent (Morisky et al, 1996). Scores from the Morisky scale will be correlated with the refill-based compliance measures such as the MPR and median gap to examine congruence between the measures.

In order to obtain the complete impact of asthma and COPD in conjunction with medication adherence, another outcome, self-reported losses in workplace productivity, was added. This component of the survey consisted of questions from the WPSI, also known as the Wellness Inventory (Goetsel et al, 2003). The WPSI consisted of questions 
that ask about the number of days with asthma or COPD symptoms in the past year, the number of workdays missed due to asthma/COPD, and the numbers of unproductive hours while at work due to the disease. In order to translate the lost workdays and unproductive work-hours into dollar metrics, a previously developed algorithm was used which incorporated average hourly wage rates for various professions in WV, obtained from the US Department of Labor (2003).

The response rate to the survey will be calculated after subtracting from the denominator the total number of respondents who requested to be removed from the study, as well as surveys that were returned due to incorrect addresses. Response rates will be calculated for each disease group (asthma or COPD) as well as for the overall Phase II sample. Difference in response rates can thus be compared between respondents with asthma and COPD. Thus, this phase of the study will give a better understanding of the impact of asthma and COPD by measuring HRQL, and workplace productivity. Phase II will also help validate the measurement of adherence, by examining the congruence between self-reported medication adherence and refill-based adherence.

\section{STUDY OBJECTIVES AND RESEARCH QUESTIONS}

\section{Phase I}

The overall goal of Phase I is to examine the relationship between medication nonadherence and healthcare utilization and costs in patients with asthma and COPD. Specific research questions that will be used to achieve this objective for asthma and COPD are stated separately. 
For Asthma:

Research Question 1: Is medication prescribing for patients with asthma in accordance with NHLBI guidelines? More specifically, among PEIA recipients with asthma, how many patients received drug therapy in either of the following four classes:

I. Short-acting beta-agonist only;

II. Combination therapy without inhaled anti-inflammatory medications;

III. At least one prescription claim for inhaled anti-inflammatory therapy (ICS, inhaled cromolyn, or inhaled nedocromil)

IV. No prescription claims for chronic pulmonary disease-related medications. Rationale: This is an exploratory question that assesses the appropriate/inappropriate prescribing of drugs in patients with asthma. Since NHLBI guidelines recommend the use of ICS as the most potent and cost-effective treatment for asthma, all patients with asthma should ideally use ICS prophylactically. Thus, the study should find that most patients with asthma use ICS as a maintenance medication on a regular basis. At the same time, since regular use of ICS reduce acute exacerbations, regular use of ICS should result in reduced utilization of short-acting beta-agonists. Ideally, patients receiving short-acting beta-agonists only, should be at a minimum.

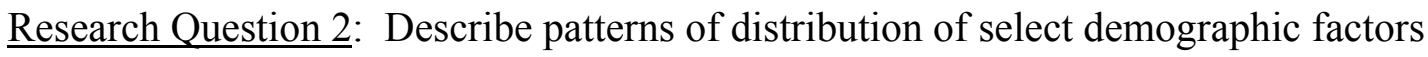
such as age, and gender in patients with asthma.

Rationale: This is an exploratory question that assesses the overall prevalence of asthma and rates of prevalence by age group and gender among PEIA covered members.

Null Hypothesis A: There is no difference in asthma prevalence by age group and gender. 
Research Question 3: What is the prevalence of nonadherence to maintenance medications among PEIA patients with asthma?

Rationale: This exploratory question assesses the extent of nonadherence to maintenance medications in the WV PEIA population, as measured from refill behavior. This research question will examine the refill patterns among PEIA patients with asthma for all maintenance medications for asthma.

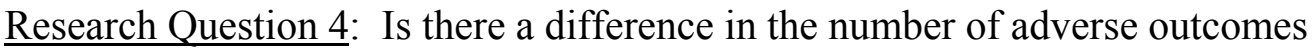
(hospitalizations and/or ED visits), outpatient visits, and hospital length-of-stay (LOS) between patients with asthma who are adherent to medications and those who are not? Null Hypothesis B: There is no difference in the number of adverse outcomes between patients with asthma who are adherent to maintenance medications and those who are not.

Null Hypothesis C: There is no difference in the number of outpatient visits between patients with asthma who are adherent to maintenance medications and those who are not.

Null Hypothesis D: There is no difference in LOS between patients with asthma who are adherent to maintenance medications and those who are not.

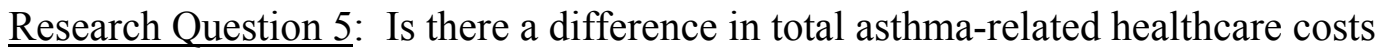
(hospitalization, ED, outpatient, and prescription costs) between patients with asthma who are adherent to maintenance medications and those who are not? 
Null Hypothesis E: There is no difference in total asthma-related healthcare costs between patients with asthma who are adherent to maintenance medications and those who are not.

Research Question 6: Is there a difference in the relative risk of adverse health outcomes such as a hospitalizations and/or and ED visits between patients with asthma who are adherent to medications and those who are not?

Null Hypothesis F: There is no difference in the risk of adverse outcomes such as hospitalizations and ED visits between patients with asthma who are adherent to maintenance medications and those who are not.

\section{For COPD:}

Research Question 1: To examine whether medication use in COPD patients is in accordance with GOLD guidelines. More specifically, among PEIA recipients with COPD, how many patients received drug therapy in either of the following two categories:

I. Bronchodilators (which consist of short-acting and long-acting betaagonists, anticholinergics, methylxanthines);

II. Corticosteroid therapy (ICS and oral corticosteroids)

III. No prescription claims for chronic pulmonary disease-related medications.

Rationale: This is an exploratory question that assesses the appropriate/inappropriate prescribing of drugs in COPD patients. Unlike asthma, GOLD guidelines in COPD do not specifically recommend the use of ICS as a prophylactic/maintenance medication. 
Thus, this research question will examine the proportion of COPD patients who received either bronchodilators or corticosteroids (either inhaled or oral).

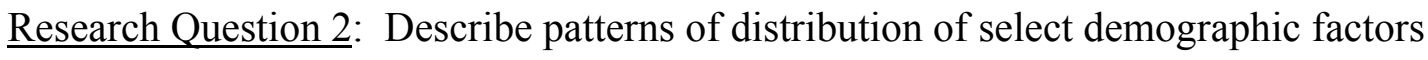
such as age and gender in patients with COPD.

Rationale: This is also an exploratory question that assesses the overall prevalence of COPD, and rates of prevalence of COPD by age group and gender among PEIA covered members.

Null Hypothesis A: There is no difference in COPD prevalence by age group and gender.

Research Question 3: What is the prevalence of nonadherence to maintenance medications among COPD patients in PEIA?

Rationale: This exploratory question assesses the extent of nonadherence to medications in the WV PEIA COPD patients, as measured by refill behavior.

Research Question 4: Is there a difference in the number of adverse outcomes (hospitalizations and/or ED visits), outpatient visits, and hospital length-of-stay (LOS) between COPD patients who are adherent to maintenance medications and those who are not?

Null Hypothesis B: There is no difference in the number of adverse outcomes between COPD patients who are adherent to maintenance medications and those who are not. Null Hypothesis $C$ : There is no difference in the number of outpatient visits between COPD patients who are adherent to maintenance medications and those who are not. 
Null Hypothesis D: There is no difference in LOS between COPD patients who are adherent to maintenance medications and those who are not.

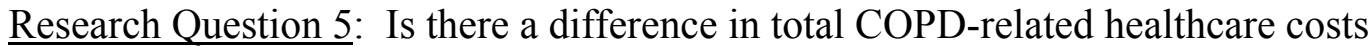
(hospitalization, ED, outpatient, and prescription costs) between COPD patients who are adherent to maintenance medications and those who are not?

Null Hypothesis E: There is no difference in total COPD-related healthcare costs between COPD patients who are adherent to maintenance medications and those who are not.

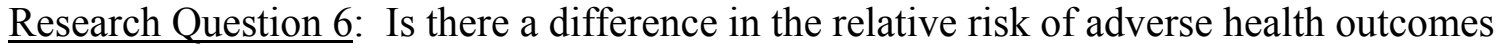
such as a hospitalizations and/or and ED visits between COPD patients who are adherent to maintenance medications and those who are not?

Null Hypothesis F: There is no difference in the risk of adverse outcomes such as hospitalizations and ED visits between COPD patients who are adherent to maintenance medications and those who are not.

\section{Phase II}

The main objective of Phase II is to examine the association between nonadherence to ICS pharmacotherapy and HRQL. The other objectives of Phase II include measuring patients' self-reported nonadherence and losses in workplace productivity due to asthma and COPD. Specific research questions are: 
For Asthma:

Research Question 7: What is the overall HRQL of patients with asthma?

Rationale: This exploratory research question will assess the domain-specific as well as overall HRQL scores for asthma patients. Absolute values for HRQL scores obtained for the WV PEIA patients with asthma will be compared with HRQL scores from other studies using the SGRQ.

Research Question 8: What is the self-reported nonadherence of PEIA recipients with asthma?

Rationale: In Phase I, adherence was measured based on prescription refill patterns from claims data. Here, patients' self-reported adherence will be measured from the questionnaire, which will include Morisky scale.

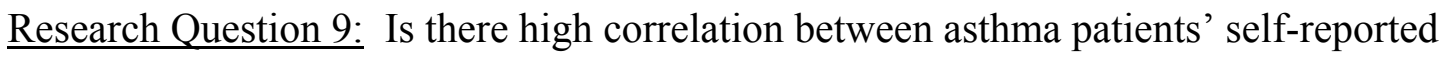
adherence and adherence calculated from prescription refills in claims data?

Rationale: A high congruence between self-reported adherence and adherence obtained from prescription refills would validate the measurement of adherence.

Null Hypothesis G: There is no significant correlation between self-reported adherence and adherence measured from prescription refills.

Research Question 10: Is there a difference in HRQL between patients with asthma who are adherent to maintenance medications and those who are not? 
Null Hypothesis H: There is no difference in HRQL between patients with asthma who are adherent to maintenance medications and those who are not.

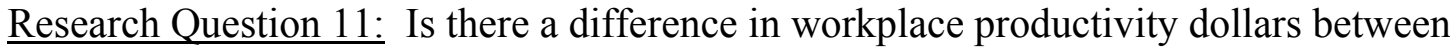
patients with asthma who are adherent to maintenance medications and those who are not?

Null Hypothesis I: There is no difference in workplace productivity dollars between patients with asthma who are adherent to maintenance medications and those who are not.

For COPD:

Research Question 7: What is the overall HRQL of patients with COPD?

Rationale: This exploratory research question will assess the domain-specific as well as overall HRQL scores for COPD patients. Absolute values for HRQL scores obtained for the WV PEIA COPD patients will be compared with HRQL scores from other studies using the SGRQ.

Research Question 8: What is the self-reported adherence of PEIA recipients with COPD?

Rationale: In Phase I, adherence was measured based on prescription refill patterns from claims data. Here, patients' self-reported adherence will be measured from the questionnaire, which will include the Morisky scale. 
Research Question 9: Is there high correlation between COPD patients' self-reported adherence and adherence calculated from prescription refills in claims data?

Rationale: A high congruence between self-reported adherence and adherence obtained from prescription refills would validate the measurement of adherence.

Null Hypothesis G: There is no significant correlation between self-reported adherence and adherence measured from prescription refills.

Research Question 10: Is there a difference in HRQL between COPD patients who are adherent to maintenance medications and those who are not?

Null Hypothesis H: There is no difference in HRQL between COPD patients who are adherent to maintenance medications and those who are not.

Research Question 11: Is there a difference in workplace productivity dollars between COPD patients who are adherent to maintenance medications and those who are not? Null Hypothesis I: There is no difference in workplace productivity dollars between COPD patients who are adherent to maintenance and those who are not.

\footnotetext{
ASSUMPTIONS

Phase I Assumptions

Phase I requires several assumptions. The first assumption concerns the validity of the paid claims data. Claims data are primarily collected for reimbursement purposes, and not research. Thus, any billing or coding errors will result in inaccuracies, which will affect the study. The important assumption with respect to claims data is the diagnosis.
} 
Since ICD9 codes will be used to classify patients as having asthma or COPD, any data entry errors will affect the study validity. WV PEIA claims data has not been used for research purposes before this project, and this study will be a test of its validity. As it is not possible to test the validity of this data, this assumption must be made. The next assumption is with respect to the measurement of medication adherence. Since adherence will be measured based on refill claims, the biggest assumption is that a refill obtained is a refill consumed. It is not possible to ascertain whether a patient used inhaled corticosteroid or any other maintenance drug appropriately. Hence, the assumption has to be made that a patient understood the correct use of inhalers, and patients who received a prescription actually consumed the medication as prescribed. Phase II Assumptions

Phase II requires certain assumptions too. First, the accuracy of the diagnosis as determined from the ICD9 code will impact who receives the survey. If the survey recipient has been inaccurately classified as having asthma or COPD, their inclusion will result in an inaccurate study group, and will also affect the survey response rate. Hence, the assumption is that PEIA members who have the ICD9 code for asthma or COPD actually have the disease. The next assumption is with respect to self-reported adherence and productivity data. Respondents are more likely to report themselves as adherent in order to provide socially desirable responses. It is however, within the scope of the study to examine the congruence between self-reported adherence, and adherence obtained from claims data. The assumption that the respondents accurately report their adherence has to be made. Similarly, some respondents are likely to underreport the number of workdays missed, or underreport the number of unproductive hours while at work due to 
the fear of being reprimanded by the employer (state). Thus, the assumption that the respondents are not underreporting the lost workdays, and unproductive work-hours has to be made.

\section{LIMITATIONS}

The limitations of this study arise from the fact that the study design is a nonrandomized, observational design, which could potentially induce selection bias. The second limitation of his study is its cross-sectional nature. Thus, the study cannot determine what outcomes resulted from poor adherence, as compared to studies where poor adherence was a marker for unmeasured causal factors. There is also endogeneity between the predictor variable - medication adherence and the outcome variables, such as HRQL and healthcare utilization and costs. Thus, it is difficult to conclude whether poor $\mathrm{HRQL}$ is a consequence of adherence, or poor HRQL resulting from the disease affected medication adherence. Also, medication adherence was measured using proxy indices. The use of these indices have limitations, the biggest assumption being that a prescription dispensed is a prescription taken, although these indices have been used and validated previously (Farris et al, 1994 and Christensen et al, 1997). This study is also subject to general limitations of claims data, such as the use of ICD9 codes, which are subject to misclassification bias. Another limitation of this study is that the exact disease severity of the patient is not known, and ICD-9 diagnosis and CPT-4 procedure codes were used as proxy. Other variables such as socioeconomic status, and physician-patient interaction, which affect medication adherence, as well as outcomes resulting from adherence were not available, and the study could suffer from potential omitted variable 
bias. Finally, the study has limited generalizability, as the results are representative of WV state employees diagnosed with asthma and COPD. This patient population is likely to be more educated, and of a higher socioeconomic status than the rest of the state residents.

\section{STUDY SIGNIFICANCE}

\section{Phase I Significance}

This study is the first study conducted in WV state employees examining the impact of medication adherence on economic outcomes such as healthcare utilization and costs in asthma and COPD. This is also the first study, which provides some estimates regarding the epidemiology and burden of asthma and COPD in state employees in WV. Finding from this study will help the state health insurance agency in designing programs to educate asthma and COPD patients about the disease. This study will provide the state data regarding the prescription use behavior among asthma and COPD patients, and help design interventions to improve physician prescribing as well as patient medicationtaking behavior. Results from this study could also act as a yardstick for guiding future health policy decisions with respect to asthma and COPD for not only the state of WV, but also other states.

\section{Phase II Significance}

This study not only examines economic outcomes, but also humanistic outcomes such as HRQL. This study will help us fathom the impact of asthma and COPD from the patient perspective, and examine changes in HRQL as a consequence of medication adherence. Literature examining the relationship between HRQL and nonadherence is 
scarce in any chronic disease, let alone asthma or COPD, and findings from this study will add substantially to the scant body of literature. A unique achievement of this study is the measurement of losses in workplace productivity due to asthma and COPD, with respect to workdays lost, as well as estimating unproductive hours while at work. These lost workdays and lost work-hours will be translated into dollar values, and will provide the true impact of the disease from the employer perspective, which in this case is the state of West Virginia. Measuring losses in productivity from an employer perspective have assumed tremendous significance in the more recent past, since patients with chronic diseases are living longer, and costly therapies are becoming more common (Kessler et al, 2001). Incorporation of this perspective takes this study closer towards capturing the complete impact of asthma and COPD.

This chapter gave a brief introduction to the problem, explained the conceptual framework, study objectives and research questions, as well as study assumptions, limitations, and its significance. The next chapter will examine the literature in detail as it pertains to medication nonadherence, and its impact on economic and humanistic outcomes. 


\section{CHAPTER TWO}

\section{LITERATURE REVIEW}

The review of the literature provides a background of the various aspects of the study involving medication nonadherence, health-related quality of life assessment, and the impact of medication use on economic and humanistic outcomes. The first section of the chapter discusses the clinical definitions, epidemiology and burden of disease, and national guidelines for the pharmacotherapeutic management of asthma and COPD. The following section examines evidence from the literature regarding the impact of prophylactic medication use on outcomes. This is followed by a discussion of the various factors that affect medication nonadherence, the prevalence of medication nonadherence, and ways of measuring adherence. The final section examines health-related quality of life in respiratory disease, with a discussion of the various instruments used for its assessment.

\section{Clinical Definitions}

Asthma is a chronic pulmonary condition, which consists of excessive airway narrowing, in response to a wide range of naturally occurring stimuli (Barbee, 1998). More specifically, asthma is characterized by reversibility of airflow obstruction, either spontaneously or as a result of therapy, in addition to bronchial hyperresponsiveness (Kamp, 1992, and Lemaneske, 1992). There is excessive mucous production, bronchial edema, and eosinophilic and lymphocytic inflammation, which is at least partially reversible. The airway inflammatory response may be divided into three phases: acute, 
late (chronic), and remodeling (Lemaneske, 1992, and Bush, 1992). The acute phase is a reaction to various stimuli - both immunologic and nonimmunologic such as allergens, exercise, irritants, and medications. The acute phase is characterized by a release of chemical mediators such as histamine, leukotrines, prostaglandins, platelet activating factor, and thromboxanes by the activated airway mast cells. These chemical mediators cause smooth muscle contraction, bronchial hyperresponsiveness, and airway wall edema. They also lead to the recruitment of other inflammatory cells and mediators (Kamp, 1992, Lemaneske 1992, Bush 1992, Oettgen and Geha, 2001).

The late phase of the asthmatic response is characterized by airway infiltration of the mast cells, eosinophils, lymphocytes, and macrophages. Subsequently, cytokines, chemokines, cationic proteins, and enzymes are released by these cells, which cause the marked inflammatory response that is typical in asthmatic airways. Airflow is compromised by bronchial hyperresponsiveness, airway wall edema, and mucous production, by the primary and secondary mediators (Kamp, 1992, and Lemaneske, 1992).

The remodeling phase is an aberrant restorative process, which results in airway modeling. Histologically, this phase is marked by basement membrane thickening and fibrosis, mucous gland hypertrophy and hypersecretion, smooth muscle hypertrophy, inflammatory cell infiltration, angiogenesis, and epithelial damage. If the inflammatory component of asthma is not adequately treated, it can be followed by this phase, which eventually results in total, irreversible loss of lung function (Kamp, 1992, Lemaneske, 1992, Bush, 1992, Oettgen and Geha, 2001). 
Based on the days/nights with symptoms, FEV1 (\% predicted), and peak flow variability, asthma has been categorized into 4 disease severity levels: Step 1 - Mild Intermittent, Step 2 - Mild Persistent, Step 3 - Moderate Persistent, and Step 4 - Severe Persistent (NHLBI Guidelines, 1997).

COPD, on the other hand, is characterized by airflow limitation that is not fully reversible (Rennard, 1998 and Pauwels et al, 2001). The airflow limitation is not only progressive, but also associated with an abnormal inflammatory response of the lungs to noxious particles and gases (Puchelle and Vargaftig, 2001). Individuals diagnosed with any of the following conditions during the course of a lifetime are categorized as suffering from COPD: asthmatic bronchitis, chronic bronchitis, chronic obstructive bronchitis, and emphysema (Pauwels et al, 2001). The clinical definition of each of these is different, although all of the above except simple bronchitis are characterized by airway flow limitation. Symptoms of COPD include dyspnea, fatigue, sputum production, or chronic cough. Unlike asthma, the airway obstruction in COPD is irreversible. It is indicated by a postbronchodilator FEV $1<80 \%$ of predicted value, in combination with FEV1/FVC $<70 \%$, confirms the presence of COPD (GOLD guidelines 1998). Based on spirometry results, FEV1/FVC values, presence/nature of symptoms, respiratory failure/signs of right heart failure, COPD patients are characterized into 4 groups: Stage 0 - At Risk, Stage 1 - Mild COPD, Stage 2 - Moderate COPD, and Stage 3 - Severe COPD (Rennard, 1998).

The differences between asthma and COPD are summarized in Table 1. 
Table 1: Clinical Differences between Asthma and COPD

\begin{tabular}{|c|c|c|}
\hline Characteristics & Asthma & COPD \\
\hline \multirow[t]{2}{*}{ Age of onset } & Usually early, variable & $\begin{array}{l}\text { Usually in the } 5^{\text {th }} \text { or } 6^{\text {th }} \\
\text { decade, some patients may } \\
\text { be diagnosed as early as } 40 \\
\text { years of age }\end{array}$ \\
\hline & Average age is 30 years & $\begin{array}{l}\text { Average COPD patient is } \\
65 \text { years of age }\end{array}$ \\
\hline Atopy & Usually present & Usually non-atopic \\
\hline \multirow[t]{2}{*}{ Symptoms } & $\begin{array}{l}\text { Dyspnea, chest tightening, } \\
\text { wheeze and cough }\end{array}$ & $\begin{array}{l}\text { Increased cough, sputum } \\
\text { and/or dyspnea }\end{array}$ \\
\hline & $\begin{array}{l}\text { When present, symptoms are } \\
\text { usually intermittent, } \\
\text { occasionally chronic and can } \\
\text { be mild, moderate or severe }\end{array}$ & $\begin{array}{l}\text { When present, symptoms } \\
\text { are chronic }\end{array}$ \\
\hline Diurnal variation & $\begin{array}{l}\text { Waking at night with cough / } \\
\text { wheeze }\end{array}$ & Nocturnal attacks rare \\
\hline Evolution & Episodic & $\begin{array}{l}\text { Slow, cumulative disabling } \\
\text { pattern }\end{array}$ \\
\hline \multirow[t]{3}{*}{$\begin{array}{l}\text { Reversibility of airflow } \\
\text { obstruction }\end{array}$} & $\begin{array}{l}\text { Airflow obstruction is episodic } \\
\text { and usually reversible with } \\
\text { therapy }\end{array}$ & $\begin{array}{l}\text { Airflow obstruction is } \\
\text { chronic and persistent }\end{array}$ \\
\hline & $\begin{array}{l}\text { FEV1 usually normal between } \\
\text { attacks and quickly improves } \\
\text { with bronchodilators }\end{array}$ & $\begin{array}{l}\text { FEV1 is persistently } \\
\text { reduced if disease } \\
\text { significant }\end{array}$ \\
\hline & $\begin{array}{l}\text { Reduction in FEV } 1 \text { may be } \\
\text { partially irreversible with long- } \\
\text { standing asthma }\end{array}$ & $\begin{array}{l}\text { With bronchodilators, } \\
\text { FEV1 usually unchanged, } \\
\text { but may improve in some } \\
\text { patients }\end{array}$ \\
\hline Chest exam & $\begin{array}{l}\text { Normal, wheezing, prolonged } \\
\text { expiratory phase }\end{array}$ & $\begin{array}{l}\text { Wheezing, hyperresonance, } \\
\text { barrel chest deformity, } \\
\text { subxiphoid apical impulse }\end{array}$ \\
\hline Role of smoking & Not directly related may & Directly related \\
\hline
\end{tabular}




\begin{tabular}{lll}
\hline \multicolumn{1}{c}{ Characteristics } & \multicolumn{1}{c}{ Asthma } & \multicolumn{1}{c}{ COPD } \\
\hline Airway hyperreactivity & $\begin{array}{l}\text { aggrate the condition } \\
\text { occurs at }</=16 \mathrm{mg} / \mathrm{mL} \\
\text { methylcholine }\end{array}$ & $\begin{array}{l}20 \% \text { fall in FEV1 does not } \\
\text { usually occur at }<25 \mathrm{mg} / \mathrm{mL} \\
\text { methacholine }\end{array}$ \\
CO diffusion (DLCO) & CO diffusion not decreased & $\begin{array}{l}\text { CO diffusion is decreased, } \\
\text { suggests impaired gas } \\
\text { exchange commonly due to } \\
\text { emphysema, should be } \\
\text { confirmed with a high- } \\
\text { resolution CT scan }\end{array}$ \\
Sputum induction for & $\begin{array}{l}\text { Eosinophils predominate in the } \\
\text { sputum of asthma patients }\end{array}$ & $\begin{array}{l}\text { Neutrophils predominate in } \\
\text { the sputum of most COPD } \\
\text { patients }\end{array}$ \\
Radiographic findings & $\begin{array}{l}\text { Often normal, may show } \\
\text { pulmonary hyperinflation }\end{array}$ & $\begin{array}{l}\text { Pulmonary hyperinflation, } \\
\text { Decreased vessels, focal } \\
\text { hyperaeration (emphysema) }\end{array}$ \\
& Elevated lung lucency & $\begin{array}{l}\text { Increased interstitial } \\
\text { markings (chronic } \\
\text { bronchitis) }\end{array}$ \\
\hline
\end{tabular}

Sources: NHLBI Guidelines, 1997, GOLD Guidelines, 1998, Jeffrey, 1999, D’Urzo, 2001. 


\section{Epidemiology and Burden}

Asthma exerts a significant burden on society, both in terms of direct as well as indirect costs. More than 15 million people in the United States are affected by asthma (Weiss and Sullivan, 2001), which is prevalent in 5-10\% of the US population (Mannino et al, 1998, and Weiss and Sullivan, 2001). The prevalence rate for asthma in 2001 was $73 / 1,000$ (CDC, 2001). Rates were higher in children $(87 / 1,000)$ than adults $(69 / 1,000)$. Among adults, females showed a 30\% higher prevalence than males, however, this trend was the opposite in children, with boys having $30 \%$ higher prevalence than girls (CDC, 2001). Prevalence rates were $10 \%$ higher in non-Hispanic blacks as compared to nonHispanic whites, and around 40\% higher than Hispanics. The outpatient visit rates were 37.9/1,000, and ED episode rates were 67/10,000 (CDC, 2001). The overall hospitalization rate among patients with asthma was $17 / 10,000$, with children and nonHispanic blacks having the highest rates for both ED episodes and hospitalizations (CDC, 2001). The overall mortality rate in asthma was 1.6 deaths per 100,000 in 2000 (CDC, 2001).

Asthma was responsible for 500,000 hospitalizations, 1.8 million emergency room visits, 6,500 deaths, 9 million lost workdays, and 10 million lost school days in 1998 (Data Fact Sheet, NIH, NHLBI, 1999). The costs of asthma to the United States range from 11.3 billion dollars (Data Fact Sheet, NIH, NHLBI, 1999) to 12.7 billion dollars (Weiss et al, 1992) per year. Hospitalizations and ED visits account for the most dollars. The average cost for an ED episode due to asthma ranges from \$234 (Stanford et al, 1999) to $\$ 457$ (Segal et al, 1995). The average hospitalization costs for asthma were $\$ 3,103$ with a mean LOS of 3.8 days (Stanford et al, 1999). There costs ranged from 
$\$ 2,000$ for patients hospitalized with mild asthma to $\$ 15,000$ for patients with more severe disease (Stanford et al, 1999). Asthma costs are highly skewed, as indicated by a population-based cost-of-illness study, which showed that less than $20 \%$ of the people with asthma were responsible for more than $80 \%$ of total costs (Malone et al, 2000). Patients with asthma having lower income and minority status experience more adverse outcomes, thereby leading to higher utilization and costs, primarily as a result of poor access to care (Druss et al, 2001).

COPD is one of the leading causes of mortality and morbidity in the United States (Hurd, 2000 and Sullivan et al, 2000). Various studies have reported the economic burden of COPD thus far (Sullivan et al, 1993, Ward et al 1994, Wilson et al, 1996, Hurd, 2000). These range from 33.2 billion (Wilson et al, 1996), to 23.9 Billion (Sullivan et al, 1993). These costs include direct as well as indirect costs. Another study showed that direct costs due to COPD were 14 billion US dollars (Hurd, 2000). Hospital use, medications, and oxygen therapy were the major cost drivers for COPD. Per capita Medicare expenditures for COPD patients were 2.4 times higher than those of other Medicare beneficiaries ( $\$ 11,841$ vs. $\$ 4901,2000$ values) (Grasso et al, 1998). The study also showed that hospital expenditures, which constituted $64 \%$ of total expenditures, were 2.7 times higher, while outpatient use was 2.2 times higher for patients with COPD. Among indirect costs, COPD was responsible for losses in employment translating into $\$ 9.9$ billion in the United States ( $\operatorname{Sin}$ et al, 2002). Another study showed that the annual cost of health services utilization in the United States was $\$ 4,119$ per patient with COPD, with indirect costs accounting for $\$ 1,527$ per COPD patient (Halpern et al, 2003). After adjusting for age and gender, COPD patients in a managed care population were 2.3 
times more likely to have been hospitalized than patients with other conditions, and those admitted had significantly longer lengths of stay (4.7 vs. 3.9 days, $\mathrm{P}<0.001$ ) (Mapel et al, 2000). Disease burden was also correlated with disease severity. Stage 1 patients experienced the lowest costs $(\$ 1,681)$, followed by Stage $2(\$ 5,037)$, and Stage 3 $(\$ 10,812)$ (Hilleman et al, 2000). Healthcare utilization in COPD patients is highly skewed, with the top $10 \%$ accounting for more than $50 \%$ of total expenditures (Ruchlin and Dasbach, 2001).

The prevalence of emphysema was 7.8/1,000 in 1994 (Wilson et al, 1994). The prevalence was higher in men $(9.34 / 1,000)$ than women $(7.33 / 1,000)$ (Murray and Lopez, 1996). The prevalence of chronic bronchitis was 54/1,000 in 1994 (Wilson et al, 2000). Another study reported the overall prevalence of COPD to be 61.9/1,000 in 1993 (NHIS, 1993). In 1996, the overall hospitalization rate was 8.9 hospitalizations/10,000, the outpatient utilization rate was 732 visits/1,000, and the emergency department use rate was 9 ED episodes/10,000 (NIH, 1995 and Wilson et al, 2000). Patients with emphysema had higher hospitalization rates compared to chronic bronchitis patients, however, chronic bronchitis patients had higher outpatient use rates. The rate of ED use was similar in both groups (NIH, 1995 and Wilson et al, 2000). Predictors of costs and utilization in COPD patients include age, health status, disease severity, physician specialty, geographic location, and type of insurance coverage (Ruchlin and Dasbach, 2001). 


\section{Prophylactic Therapy in Asthma and Chronic Obstructive Pulmonary Disease}

In 1997, the National Asthma Education and Prevention Program Expert Panel Report 2 was released by the National Heart, Lung and Blood Institute, which consisted of published guidelines for the management of asthma (NHLBI Guidelines, 1997). This report stated that ICS were the most potent and effective long-term control medications for asthma. The stepwise guidelines for the treatment of asthma are as follows: $\underline{\text { Stepwise approach to pharmacologic therapy for asthma }}$

Step 1: Occasional use of relief bronchodilators

Step 2: Regular inhaled anti-inflammatory medications

*Initial trial of sodium cromoglycate or nedocromil sodium

*If unsuccessful, budesonide or beclomethasone dipropionate, $200-800 \mu \mathrm{g} / \mathrm{day}$

Step 3: High-dose inhaled glucocorticosteroid (e.g., budesonide 1200-1600 $\mu \mathrm{g} /$ day)

Step 4: High-dose inhaled glucocorticosteroid and regular bronchodilators

*Oral slow-release theophylline

*Possible addition of inhaled ipratropium bromide or long-acting $\beta 2$-agonist (salmeterol)

Step 5: Long-term alternate day or daily oral prednisone

Step 6: When stepping down, review treatment every 3 to 6 weeks

These guidelines state that the most cost-effective way to reduce acute symptoms with anti-inflammatory therapy is a short course $(<=$ one week $)$ of a systemic corticosteroid, followed by chronic use of an ICS. The guidelines recommend that ICS should be used in conjunction with a long-acting beta-agonist. In patients with COPD, the use of ICS has been underscored in many studies, although consensus guidelines have not been developed (van Schyak et al, 1996, Rowe et al, 1992, Hatoum et al, 1994). 
Overall, pharmacotherapy in COPD is used to improve symptoms and/or disease complications. None of the existing medications for COPD have been shown to modify the long-term decline in lung function, which is a characteristic of COPD. An important component of COPD treatment is smoking cessation (Pauwels et al, 2001).

The 1997 NHLBI asthma guidelines recommend the use of ICS for control of bronchial asthma in patients already receiving systemic corticosteroids, and those patients who are unresponsive to nonsteroid regimen. They are not recommended for the acute relief of asthma, which can be controlled by bronchodilators, and other nonsteroid medications and in patients who require systemic corticosteroid treatment infrequently, or in the treatment of non-asthmatic bronchitis. It is also contraindicated in primary treatment of status asthmaticus when intensive measures are required, when there is hypersensitivity to any ingredient, or when there are systemic fungal infections and persistently positive sputum cultures in patients for candida albicans. Localized fungal infections have occurred in patients who are on corticosteroid inhaler pharmacotherapy.

Clinical responsiveness to ICS is highly dependent on proper administration. Bai (1995) reviewed ICS from a clinical and economic standpoint, and suggested that the choice of inhaled glucocorticosteroid for therapy depends upon a number of factors such as systemic bioavailability, delivery device, patient acceptance, and most importantly, cost. High cost of ICS makes them cost-prohibitive. The use of these medications, however, results in cost savings to the overall healthcare system due to reduced work absences, losses in productivity, reduced ED and hospitalizations, and bronchodilator use. Also, as shown in the Sasketchewan study, premature death is lower in patients taking ICS. Thus the use of ICS is clearly justified, according to Bai (1995). 


\section{Clinical Efficacy of Inhaled Corticosteroids: A Review of Meta-Analyses}

Rowe and colleagues (1992) conducted a meta-analysis to examine the effect of steroid therapy on pulmonary function, admission rates and relapse rates in patients presenting with acute exacerbations of asthma. The study, which used results from 30 randomized clinical trials, found that the use of steroids, early in the treatment of asthmatic exacerbations significantly reduced admission in adults (OR $0.47,95 \% \mathrm{CI}$ $0.27-0.79)$ and children (OR $0.47,95 \%$ CI $0.06-0.42)$. They also found that corticosteroids were effective in preventing the rates of relapse in outpatient treatment of asthmatic exacerbations ( $\mathrm{OR}=0.15,95 \%$ CI 0.05-0.44) (Rowe, 1992).

Another meta-analysis conducted by Hatoum and colleagues (1994) to determine the role of ICS in the treatment of mild chronic asthma used peak expiratory flow rates (PEFR) as the outcome measure. Using PEFR as the objective endpoint for effect size, the authors found that effect sizes ranged between 0.41 to 0.89 , and the overall weighted average effect size for PEFR was 0.59 . The statistical significance of the results enabled the authors to conclude that ICS were beneficial in the treatment of mild chronic asthma.

In patients with COPD, recent evidence shows that although ICS do not slow the progression of the disease, they do reduce exacerbations, mortality, as well as selfperceived health status and HRQL. In a retrospective analysis of managed care claims data, it was shown that COPD patients receiving ICS and/or salmeterol experienced 14 percent lower mortality rates than patients who did not receive the drugs (Mapel et al, 2002). Van der Valk and colleagues (2002) showed that COPD patients receiving fluticasone experienced a significant reduction in acute exacerbations, as well as a 
significant improvement in HRQL, as compared to patients not receiving fluticasone. Unlike asthma, where bronchial hyperresponsiveness is related to severe inflammation, in COPD, bronchial hyperresponsiveness is a function of the degree of existing airway obstruction. The GOLD guidelines released by the NHLBI recommend that inhaled glucocorticosteroids should only be prescribed for symptomatic COPD patients with a documented spirometric response to glucocorticosteroids, or for those with an FEV1 $<50 \%$ predicted and repeated exacerbations requiring treatment with antibiotics and/or oral glucocorticosteroids (Pauwels et al, 2001). A review of 14 studies conducted by van Schyak and colleagues (1996), indicated that the use of ICS in COPD seems to have some beneficial effects on lung function.

\section{Prophylactic Pharmacotherapy and Economic Outcomes}

Various studies have documented the beneficial effects of prophylactic pharmacotherapy in chronic pulmonary disease. Huse and colleagues (2000) examined the impact of choosing ICS versus long-acting bronchodilators on total costs. They found that patients who used inhaled medications only, experienced a statistically significant decline in costs in the year following the treatment as compared to patients who used bronchodilators only, as maintenance therapy ( $\$ 93$ vs. $\$ 76 ; p<0.0001$ ). These costs were age-adjusted, and both groups had similar costs during the baseline year. However, the study was nonrandomized, and did not explicitly measure adherence. The use of longacting bronchodilators over inhaled medications could be preferential as well. Stempel and colleagues (1997) have indicated that specialists are more likely to prescribe inhaled corticosteroids as compared to generalists. If asthma patients were treated by a specialist, 
$39 \%$ received a prescription for inhaled corticosteroid; whereas if the treating physician was a generalist, only $22 \%$ were prescribed an inhaled corticosteroid. These trends continued over time. Another study by $\mathrm{Wu}$ and colleagues (2001) showed that asthma patients treated by pulmonologists were more likely to be treated with an inhaled corticosteroid than those treated by a generalist. These patients were also more knowledgeable about the disease, and experienced fewer ED visits and hospitalizations. Thus, physician specialty should be included as a covariate in studies examining asthma outcomes. In a managed care population consisting of Medicare, Medicaid, as well as commercial enrollees, only $26 \%$ of the enrollees received an inhaled corticosteroid. Around 33\% of the enrollees utilized only short-acting beta-agonists. These enrollees experienced significantly higher incidence of adverse outcomes such as ED visits and hospitalizations, as compared to those receiving inhaled corticosteroids (Nestor et al, 1998).

In an analysis of the Saskatchewan Health databases, Suissa and colleagues (2000) carried out a population-based cohort study of patients using anti-asthma drugs. The relative risk of mortality was calculated after adjustment of various covariates such as age, gender, and medication use in the year before the index date, and number of hospitalizations two years before the index date. The cases experienced a $21 \%$ lower mortality rate with each additional canister of inhaled corticosteroid used $(\mathrm{RR}=0.79 ; 95 \%$ CI 0.65 to 0.97$)$. Also, the death rate was higher in the first three months after therapy discontinuation, as compared with those who continued to take the inhaled medication. Thus, the regular use of inhaled corticosteroids was associated with a reduced risk of mortality due to asthma. Ernst and colleagues (1992) conducted a nested case-control 
analysis to examine the risk of fatal and near-fatal asthma events in relation to ICS use. After adjustment for 'other' medication use, and markers of asthma severity such as prior hospitalizations and visits to specialists, the study found a $90 \%$ reduction in the probability of a fatal or a near-fatal event in patients receiving ICS compared to those who did not (OR $0.1 ; 95 \%$ CI $0.02-0.6)$.

Inhaled steroid dispensing was associated with a 50\% decrease in the risk of hospitalization, after adjusting for age, race and other asthma medications in a managed care population (Donahue, 1997). Regular use of inhaled corticosteroids has also been associated with a significant reduction in the rate of readmission in asthma patients (Blais et al, 1998). The study compared first-time users of ICS with first-time users of theophylline. The study found an $80 \%$ reduction in hospital readmission rates in the group receiving inhaled corticosteroids; although disease severity, age and gender were potential confounders. Another cohort study by Blais and colleagues (1998) examined the impact of inhaled corticosteroid use on the risk of readmission for asthma. This study found that although inhaled corticosteroids reduced the risk of readmission due to asthma by $40 \%$, the positive effects of therapy disappeared after 6 months of use. The authors attributed this disappearance of the beneficial effect to confounding by disease severity. Most studies examining outcomes in asthma focus on short-term effects of pharmacotherapy. A study by Suissa et al (2002) focused on long-term hospitalization outcomes in patients receiving ICS therapy. Regular use of ICS was associated with a $31 \%$ reduction in asthma-related hospitalizations, and a 39\% reduction in readmission rates. This reduction during the first four years of follow-up was maintained in the long- 
term; thereby resulting in a reduction of five asthma hospitalizations per 1,000, and 27 readmissions $/ 1,000$.

Selroos and colleagues (1995) examined the effects of introducing early versus late ICS treatment in patients with mild or moderate asthma. The use of ICS for more than one year resulted in significant improvements in PEFR and FEV1. However, these improvements were the highest among patients experiencing asthma symptoms for less than two years. In patients experiencing symptoms for more than two years, the improvement in FEV1 and PEFR was highest after one year of ICS therapy, but decreased thereafter. Thus, early introduction of ICS therapy was associated with improved clinical outcomes, which supports the NHLBI guidelines recommending early use of ICS therapy following asthma diagnosis. These findings were further reinforced by Selroos and colleagues (2004). They compared an early treatment group with a delayed treatment group with varied symptom durations. Early treatment with ICS was associated with better airway function and asthma control.

The positive impact of ICS therapy has been seen in children as well. Balkrishnan and colleagues (1998) found that the introduction of prophylactic ICS pharmacotherapy was associated with significant clinical and economic benefits in Medicaid-enrolled children. Regular inhaler refills were associated with a significant reduction on total healthcare costs in asthma patients. Another study by Balkrishnan et al (1998) found a $50 \%$ reduction in the rate of hospitalizations, and a $26 \%$ reduction in outpatient use, after initiation of ICS pharmacotherapy, in a Medicaid population. The study also found a $24 \%$ decrease in total healthcare costs per asthmatic per month as a result of the introduction of ICS pharmacotherapy. 
Rutten van Molken et al (1995) examined the costs and effects of combined bronchodilator and ICS pharmacotherapy in asthma and COPD patients. They found that the addition of ICS to $\beta$-agonist pharmacotherapy lead to significant improvements in respiratory function and restricted activity days. Although the cost of ICS/bronchodilator therapy was almost twice that of bronchodilator/anticholinergic therapy, this resulted in savings in other healthcare costs by $\$ 175$. Although the ICS/bronchodilator therapy increased net costs by $\$ 201$ per patient per year, this translated into a significant reduction in the number of restricted activity days due to improved lung function. Thus, the high costs of therapy were offset by improvement in overall productivity resulting in a net societal savings of $\$ 42$ per day. Another study by Smith and colleagues (2001) supported these findings. They found that the increase in costs due to ICS was offset by the reduction in acute care-related medical visits.

Balkrishnan and Christensen (2000) examined the impact of medication adherence to ICS pharmacotherapy in patients with both asthma as well as COPD, on health services utilization and costs. This study used the Med-Total approach to measure adherence, where adherence is defined as the days of prescription supply dispensed divided by the days between prescription refills. This study conducted in elderly patients with asthma, found that poor medication adherence was associated with a $5 \%$ decrease in physician visits $(\mathrm{p}<0.05)$, whereas better medication adherence was associated with a $20 \%$ decrease in annual hospitalizations, after adjusting for respiratory disease-specific severity using proxy measures based on ICD-9 codes, as well as overall disease severity using Charlson's comorbidity index. Overall, studies have shown that adherence to 
NHLBI guidelines, which recommend the use of ICS as the most potent and costeffective prophylactic medication for asthma, resulted in better outcomes.

\section{Conceptual Definition of Medication Adherence}

Medication adherence is defined as the "extent to which a person's medicationtaking behavior coincides with medical advice." (Haynes et al 1979) Adherence is a newer term, which suggests a more proactive role on the part of the patient to adhere to recommendations of their physicians, vis-à-vis the more traditional term "compliance", which implies that the patient passively submits to the prescriber's advice towards a medication regimen (Fish and Lung, 2001). Full medication adherence occurs when all the instructions of the prescriber are followed. Possible signs of nonadherence include patients' failure to fill the prescription, loss to follow-up of the patient for further evaluation, and zero serum-drug concentrations. Partially adherent patients take their drug more or less often than prescribed and/or take their doses erratically.

Fincham (1995) has suggested a continuum to be considered when examining the use of medication adherence. The continuum of adherence comprise of four points: Initial Adherence. The patient receives a written prescription, has the prescription phoned to a pharmacy or transferred to it, but does not wait or return to pick up the filled prescription. This group also includes patients who do not present written prescriptions for filling the medication.

Partial Adherence. The patient takes the dispensed medication at a level less than the prescriber or the dispenser intended. 
Adherence. The process of adhering with a prescribed regimen precisely as the prescriber or dispenser has intended.

Hyperadherence. The case where a patient takes a prescribed or dispensed medication at a level over and above the recommended or intended dosing level.

Fincham also indicates that the patient moves from one type of adherence to another at varying times and with different dosage regimens.

There are some concerns with measuring adherence: (1) adherence follows along a continuum from total adherence to total nonadherence, (2) lack of consistency in a given patient, and (3) measurement of adherence may be dependent on accurate provision of information by the patient, depending on the method used to assess adherence (Fish, 2001).

The importance of pharmacologic therapy in the overall treatment regimen cannot be undermined. Lack of compliance with prescribed drug regimens costs the United States $\$ 100$ million annually (National Pharmaceutical Council, 1992). In general, nonadherence have at least 3 deleterious effects: (1) reduction of effectiveness, (2) increased occurrence of adverse effects due to uncontrolled disease, and (3) in clinical trials, it can result in masking of side-effects due to the drug in question.

\section{Factors Affecting Medication Adherence}

The factors that impact adherence / nonadherence can be grouped into three broad categories:

1. Treatment-related factors: 
a. Regimen complexity: Adherence rates are directly correlated with dosing frequency. Adherence rates drop drastically at higher dosing frequencies (Greenberg, 1984 and Tashkin, 1995). Patients are more likely to be adherent in a treatment plan that is simple.

b. Mode of administration: Studies have shown that adherence varies based on methods of administration. Some patients prefer using oral medications as compared to using inhalers, because they feel that inhalers are less convenient, and at the same, they feel uncomfortable about using inhalers in public (Osman, 1996 and Ringdal et al, 1998). Overall, adherence rates have been higher with oral versus inhaled therapy (Fish and Lung, 2001)

c. Cost: The nature of prescription drug coverage can significantly impact the probability of obtaining prescriptions. Patients who do not have adequate prescription drug coverage should be prescribed economically efficient prescriptions.

d. Prolonged / Prophylactic therapy: Asthma treatment also consists of longterm use of controller medication, which acts prophylactically, and prevents the occurrence of asthma symptoms and acute exacerbations. Patients are required to take these medications daily, even when symptoms are absent. If patients no longer think they need medications since symptoms are absent, it can lead to adverse outcomes, which can be attributed to nonadherence.

2. Patient-related factors: 
a. Knowledge of disease/treatment: Patients who do not understand the importance of long term controller medication often are likely to have poorer adherence and worse outcomes. Patients should also understand the pathophysiology of the disease, and its symptoms. Patients should know that inflammation is the primary mechanism that occurs in asthma, and that asthma is a chronic disease. Patients should also be educated about asthma symptoms and what happens during an asthma attack. Patients should be able to recognize an exacerbation and take treatment accordingly.

b. Disease severity: Adherence to medications has been lower in patients with mild asthma or severe asthma. Thus, a curvilinear relationship exists between severity and adherence. A possible explanation could be that patients with mild asthma do not feel the need for controller therapy and are hence nonadherent. This could potentially lead to acute exacerbations, and more patients in this phase feel that taking medications is hopeless, again resulting in poor adherence (Leidy, 1995). Another explanation is that severe patients have worse HRQL, which impacts self-efficacy resulting in nonadherence.

c. Self-efficacy: Adherence will tend to be lower in patients with lowefficacy. It has been shown that patients are more likely to be adherent if their perception regarding their own well-being is positive (Peters et al, 1995, and Leidy, 1995). Apter and colleagues (1998) examined patient characteristics that may influence adherence with twice daily inhaled 
steroid regimens. The study used electronic monitoring to measure adherence, and found that the factors associated with poor adherence were poor physician-patient communication, lack of education, income, and language barriers. Locus of control, however was not a significant predictor of adherence. Self-efficacy can also be a function of other comorbidities. It has been shown that clinically depressed patients are three times as likely to be nonadherent with pharmacological therapy than patients without depression (Dimatteo et al, 2000).

3. Healthcare professional-related factors:

a. Communication: Good communication between the physician and the caregiver improves adherence, as it makes the patient a part of the decision-making process. If the physician is disinterested, adherence rates will drop.

b. Regular access to care: If the patient is seeing a different physician with each visit (rotating caregivers), adherence rates will drop. Patient confidence in both treatment as well as the physician, has been shown to drop when patients are seen by more than one primary care physician (Bender, 2002).

\section{Measurement of Adherence}

The measurement of adherence has always been a challenge to researchers as well as practitioners. There is no gold standard to measure adherence or the lack of it, and there is controversy with regards to which measure provides the best estimate of a 
patient's medication-taking behavior. Some reviews have suggested that biologic assays are the most accurate measure of patient nonadherence, followed by pill counts, and that self-reports are the most inaccurate (O'Hanrahan and O'Malley, 1981, Evans and Spelman, 1983, Eraker et al, 1984, Rudd, 1991). Other researchers have suggested that self-reported adherence is the most valid measurement, but only when the interview is conducted in person (Fletcher et al, 1979, Stewart, 1987, Landry et al 1988).

Pharmacy claims databases have also been extensively used to measure adherence as defined by the frequency of refills (Farris et al, 1994, Christensen et al, 1997, Steiner and Prochazka, 1997, Grymonpre et al, 1998). Steiner and Porchazka (1997) conducted a literature review of studies measuring refill compliance. They concluded that refill compliance is a useful indicator of patient adherence when direct measurement is not practical. Another study by Steiner and colleagues (1988) concluded that refill-based measures correlated well with disease-specific drug effects, and were therefore valid measures of compliance. Farris et al (1994) concluded that while refill compliance measured using 'days supply' as indicated in prescription claims data was a good measure of compliance, they indicated that refill measures were better indicators of population-level adherence, rather than individual patient-level adherence.

Some studies have compared different methods of assessing adherence. Choo et al (1999) compared adherence from patient self-reports with pill counts, and pharmacy records. They concluded that adherence measured from pharmacy records were a valid measure of adherence. Another study by Erickson and colleagues measured nonadherence in asthma patients who used metered dose inhalers (MDIs) with that obtained from pharmacy claims data. The study found a statistically significant 
agreement $(75.5 \% ; \mathrm{p}<0.01)$ between adherence reported from pharmacy claims and adherence obtained from the self-reported questionnaire.

\section{Morisky Medication Adherence Scale}

The Morisky Medication Adherence scale (Morisky et al, 1986) has been extensively used to measure self-reported adherence in various disease conditions. This scale is a generic, 4-item scale which measures adherence on a continuous scale of 0 to 4 , where 0 represents most adherent and 4 represents most nonadherent. Specifically, patients responding with a score of 0 are classified as 'high adherent', patients having scores from 1 to 2 are classified as 'medium adherent', and those having scores greater than or equal to 3 are classified as 'low adherent.' This scale has been shown to be both reliable and valid, as indicated by its high internal consistency (Morisky et al, 1988). The scale also demonstrated good concurrent as well as construct validity, and reasonable predictive validity (Morisky et al, 1988). The Morisky scale has been used to measure self-reported adherence in patients with various disease conditions such as hypertension, HIV, tuberculosis, as well as measure adherence to antibiotic therapy (Morisky et al, 1990, Huang and Morisky, 1999, Gao and Nau, 2000, Ward et al, 2000, Pratt et al, 2001, Busto et al, 2001).

\section{Workplace Productivity Losses in Asthma and COPD}

Both asthma and COPD being chronic diseases significantly impact workplace productivity in terms of days absent from work (absenteeism), as well as reduced effectiveness while at work due to the disease (presenteeism). Asthma is a condition that 
affects adults of working age. Patients with asthma experienced around 11 mean absenteeism days, and losses in presenteeism equivalent to almost 18 days per year (Wang et al, 2003). Among indirect costs, asthma resulted in a loss of work-days, which translated into $\$ 1,550$ per asthmatic annually (Cisternas et al, 2003). The study showed that asthma resulted not only in decreased effectiveness while at work, but was also responsible for loss of employment (Ungar and Coyte, 2000 and Blanc et al, 2001).

COPD, although more prevalent in the elderly, can also affect the working age population. As indicated in the 1995 National Ambulatory Medical Care Survey, almost three-fourths of COPD patients were under 65 years of age, and were accountable for $67 \%$ of COPD-related office visits, and $43 \%$ of all hospitalizations (Strassels et al, 2001). COPD was responsible for losses in employment translating into $\$ 9.9$ billion in the United States (Sin et al, 2002). Another study showed that the annual cost of health services utilization in the United States was $\$ 4,119$ per patient with COPD, with indirect costs accounting for $\$ 1,527$ per COPD patient (Halpern et al, 2003). Patients with emphysema experienced around 19 mean absenteeism days, and losses in presenteeism equivalent to almost 28 days per year (Wang et al, 2003).

\section{Measurement of Losses in Workplace Productivity}

Several instruments have been used to measure losses in productivity due to asthma and COPD. These instruments include national surveys such as the National Medical Expenditure Survey (Strassels et al, 2001), the Third National Health and Nutrition Examination Survey (NHANES III) (Sin et al, 2002), the World Health organization's Work and Health Performance Questionnaire (Wang et al, 2003), the 
American Productivity Audit (APA) (Stewart et al, 2003), the Confronting COPD Survey (Halpern et al, 2003), the McArthur Foundation Midlife Development in the United States (MIDUS) (Kessler et al, 2001), and the National Comorbidity Survey (Kessler et al, 2003). Also, other instruments such as the Work Productivity and Activity Impairment Questionnaire (WPAI) (Reilly et al, 1996), the Worker Productivity Index (WPI) (Burton et al, 1999), the Work Limitations Questionnaire (WLQ) (Lerner et al, 2001), the Endicott Work Productivity Scale (Endicott and Neel, 1997), and the Work Productivity Short Inventory (WPSI) (Goetsel et al, 2003) have also been used.

\section{Work Productivity Short Inventory (WPSI)}

For the measurement of losses in productivity due to asthma and COPD in this study, the WPSI, also known as the Wellness Inventory will be used (Goetsel et al, 2003). This instrument was originally created to measure absenteeism and productivity losses at work due to eleven chronic diseases including respiratory illnesses. The instrument was chosen since it has shown good evidence of reliability, and has low respondent burden (Goetsel et al, 2003). The instrument asks three questions to measure the impact of disease on lost productivity. These include the number of days with disease, days missed from work due to disease, and number of unproductive hours while at work due to the disease. The creators have also developed an algorithm to convert the losses in workdays and work-hours into dollar metrics. Since the instrument included only three questions to measure productivity losses, the low respondent burden was especially appealing with regards to choice of the instrument, since productivity was just one component of the survey, in addition to measuring HRQL, and self-reported adherence. 


\section{Health-Related Quality of Life in Asthma and Chronic Obstructive Pulmonary}

\section{Disease}

HRQL has been defined as "the functional effects of illness and its consequent therapy upon a patient, as perceived by the patient." (Schipper et al, 1996). Although there is a considerable amount of economic and epidemiologic evidence of the impact of asthma and COPD, relatively little is known regarding its impact on HRQL. This gap needs to be addressed since both asthma and COPD have a considerable impact on physical, psychological and social domains of HRQL. The chronic nature of asthma and the unpredictability and uncertainty of acute episodes can substantially lower patients' HRQL. The presence and fear of symptoms may cause patients with asthma to avoid physical activities and social situations, which they might otherwise enjoy. More specifically, patients with asthma are more concerned about restrictions in daily activities such as performing household chores, gardening and participating in sports (Nocon and Booth, 1991). Nocturnal symptoms in asthma can disturb sleep and rest patterns, which can have a detrimental impact of overall vitality and energy levels. Managing this chronic condition can drain an asthmatic physically, mentally as well as financially.

\section{Correlation between HRQL and Disease Severity}

Although one would expect a high correlation between disease severity and HRQL, this is not always true. HRQL is subjective and varies from individual to individual. Thus, although conventional measures such as spirometry, medication use, symptom severity, airway hyperresponsiveness and sputum analysis provide valuable clinical information, they tell us very little about functional impairments that are 
important to asthma patients in their daily lives. In fact studies have shown that there is a very poor correlation between clinical outcomes such as FEV1 and PEFR, and HRQL. The correlation between spirometry and HRQL is also weak (Jones et al, 1989, and Jones et al, 1992). Both generic instruments such as the SF-36 (Bousquet et al, 1994), as well as disease-specific instruments have found poor to moderate relationships with clinical indicators in chronic respiratory disease (Juniper et al, 1995, , van Molken et al, 1995, van der Molen et al, 1997, Leidy and Coughlin 1997). There could be several possible explanations for this weak correlation. First, HRQL assesses patient perceptions of the condition and life experience over time, whereas pulmonary function is a static indicator; hence the low correlation with HRQL in a condition like asthma characterized by variability. Second, the nature of the relationship between HRQL and pulmonary function may be nonlinear, both cross-sectionally and longitudinally (Jones, 1995).

In COPD, unlike asthma, there may be no sudden episodes of acute chest tightness, dyspnea, or wheezing, that might catch the patients' attention. Breathlessness may be noticed by the patients during recreational activities or exercise. This disturbance of physical activity causes the patients to be handicapped since their impairment constrains the activities they wish to perform. Not only breathlessness, but cough and sputum production can have physical, social and emotional effects on patients. Anxiety and depression levels are usually higher in COPD patients. A significant correlation has been found between loss of vital capacity and depression in patients with COPD (Jones, 1995). In order to capture the overall effect of COPD, disturbances in a range of different aspects of disease activity, disability and distress should be examined. 


\section{HRQL Measurement: Asthma versus COPD}

An instrument that measures HRQL in patients with asthma will differ from a COPD instrument since both diseases impact different domains (Jones, 1995, and Janssens, 2001). For example, the domains that are most likely to be affected in asthma include symptoms, activity limitations, and emotional disturbances (Mahler, 2000, and Jones, 1995). In COPD patients, dyspnea (Mahler and Mackowiak, 1995, Breslin et al 1998, Hajiro et al, 1999) and days/nights disturbed by respiratory complaints are the domains that are more likely to be affected (Breslin et al, 1998). In fact, the impact of dyspnea in HRQL in COPD is so significant that some researchers have recommended using extent of dyspnea by itself to categorize COPD patients based on disease severity (Hajiro et al, 1999). Also, chronic cough and sputum production in COPD patients are likely to have physical, social and emotional effects on patients (Jones, 1995). Anxiety and depression levels will tend to be higher in COPD patients as well (Jones, 1995). Due to the chronic nature of COPD, the impact of reduced vital capacity on social and emotional dimensions are likely to be significantly higher in COPD; as compared to asthma, where due to the episodic nature of the disease, temporary loss in activity limitations, and impact of symptoms will be greater (Jones, 1995 and Janssens, 2001). Also, when the impact of disability is captured, it can mean differently to a patient with asthma versus a patient with COPD. An asthmatic is likely to be younger, whereas a COPD patients is likely to be much older, retired, and disability would be a function of the inability to perform leisurely activities, as compared to a patient with asthma, where the disability would be a function of the inability to perform daily activities or loss in productivity at work (Jones, 1995 and Wijnhoven et al 2001). Thus a comprehensive 
picture of HRQL in COPD would consist of measuring the following dimensions: disease activity, disability and distress, as compared to asthma where the focus is on symptoms and activity limitations. Some domains recommended for measuring COPD HRQL are: cough, wheeze, walking distance, disability and anxiety level. Domains recommended for asthma include: symptoms (shortness of breath, chest tightness, wheeze, cough), emotions, activities (sports, social activities, pets, housework), environment (dust, cigarettes, air pollution, pollen). Cigarette smoking is another factor that can affect asthma and COPD patients differently. These are some of the considerations that would go in choosing domains and specific items for measuring HRQL in asthma/COPD patients. In order to distinguish between asthma and COPD patients, questions related to the following factors should be included: age at diagnosis, onset, nature of symptoms (such as cough, symptoms, nocturnal occurrences), prevalence of smoking, type of medications used, and frequency of medications. Explicit questions such as: Have you ever been told by your doctor that you have asthma, will be used with a dichotomous Yes/No response. These questions would help us determine which patients have asthma and / or COPD if diagnosis is not already known.

\section{HRQL Measurement: Choice of Scales}

The nature of the scales used in the instrument would depend on the intended use: whether the instrument is designed to be used for discriminative (or cross-sectional), evaluative (or longitudinal) or predictive purposes. A discriminative instrument would consist of mainly yes/no type of responses (Juniper, 1995 and Sen et al, 1999). For evaluative instruments, individual items have to be sensitive to change. Scores should 
change when clinically or humanistically important changes occur. Items with 5, 7, or 9 response options or visual analog scale (VAS) can be used in an evaluative instrument (Juniper, 1995). For a predictive instrument, a response set that maximizes correlation between scores and a criterion measure should be used (Juniper, 1995).

\section{HRQL Measurement: Psychometric Stability}

Reliability and validity testing can also change based on the intended purpose. An instrument that is used for discriminative purposes should have established reliability, cross-sectional validity (Guyatt et al, 1992), whereas an evaluative instrument should have reliability and longitudinal validity (Juniper, 1995 and Sen et al, 1999). Reliability between patients would be measured by assessing the signal to noise ratio where signal represents between group differences, and noise represents within group differences (Streiner et al, 1989 and Juniper, 1995). An indicator of this would be the ICC, or the intra class coefficient, which related the between patient variance to the total variance (Juniper, 1995). Cronbach's alpha on the other hand, measures the internal consistency, where it measures the correlation between an individual item and how it correlates with the overall domain (Juniper, 1995). Cross sectional construct validity would be measured by looking at the correlations between the HRQL scores, and indicators of asthma / COPD severity, such as FEV1, or the walk-test, or dyspnea, or correlations with the Sickness Impact Profile (SIP) (Juniper, 1995). For evaluative instruments, longitudinal construct validity and responsiveness have to be established in addition to reliability. In order to measure longitudinal construct validity, one would measure the changes in HRQL scores with corresponding changes in relevant outcome measures, such 
as severity or symptom measures in a predicted manner (Juniper, 1995). In order to assess responsiveness, one would measure changes in HRQL scores with corresponding changes in disease over time. Thus the instrument can be readministered in the same sample after a certain time period (say 1 week), and changes in HRQL should correspond with changes in disease severity over time, for the instrument to satisfy the criteria of responsiveness (Guyatt et al, 1987 and Juniper, 1995). The magnitude of the responsiveness can be measured by looking at the effect size: magnitude of change to the random variability in scores. On the other hand, if the severity has not changed over time, and the instruments is readministered, and HRQL scores do not change, this does not indicate presence or absence of responsiveness, it does however, indicate that the instrument is reproducible (test-retest reliability).

\section{HRQL Measurement: Clinical versus Statistical Significance}

Even if the difference in HRQL scores between groups is statistically significant, it may not necessarily be clinically significant (Juniper, 1994 and Juniper, 1998). To interpret HRQL scores, two approaches can be used: the distribution-based approach, and the anchor-based approach (Lydick and Epstein, 1993, and Juniper 1999). The distribution-based approach would use the statistical significance, based on the effect size, which is derived from the magnitude of change and variability in stable patients. This approach does not indicate whether the change is clinically relevant to the patient (Juniper, 1998 and Juniper, 1999). Clinical relevance can be captured by the anchorbased approach, which defines the minimally important difference (MID) as 'the smallest difference in score in the domain of interest which patients perceive as beneficial and 
would mandate, in the absence of troublesome side-effects and excess costs, a change in the patients management' (Juniper, 1999). In addition the number needed to treat (NNT) can be calculated if the MID is known. The NNT is the number of patients who need to be treated with the new intervention for one patient to have a clinically important change over and above which he/she would have experienced with the control intervention (Juniper, 1998 and Guyatt et al, 1998).

\section{HRQL Instruments Used in Asthma and COPD}

A range of instruments have been used to measure HRQL in both adults and children with asthma. Some instruments have better psychometric properties than others, although not a single instrument can be psychometrically perfect. There are two types of HRQL instruments used in asthma and COPD: generic and disease-specific. Generic questionnaires have been designed for use in patients with various diseases. Among them, the most commonly used and validated in adults are the Sickness Impact Profile (SIP) (Bergner et al, 1981), the Medical Outcomes Study Short-Form - 36 (SF-36) (Stewart et al, 1988), the Nottingham Health Profile (Hunt et al, 1980), the McMaster Health Index questionnaire (Sackett et al, 1977), and the Dartmouth COOP Charts (Nelson et al, 1987). Each instrument attempts to measure HRQL in different ways. The SF-36 has 3 major attributes: (functional status, well-being and overall evaluation of health). These 3 attributes or domains are not combined into one overall score. On the other hand, the SIP has 2 subscales: physical and psychosocial, which can be combined into one overall score. 
The major limitation of generic instruments is that they may not capture areas of impairment that may be relevant to the particular disease. For example, the SIP tends to focus on more severe impairments such as feeding and dressing. that may not be relevant to a disease like asthma. Also, the responsiveness of generic instruments to small but clinically important changes tends to be very poor, thereby potentially restricting their use in clinical trials.

Utility-based instruments measure the value or utility the individual or society places on various health states. These scales provide a number representing HRQL from zero to one, and they meet the assumptions for utility theory. These instruments are widely used by economists to conduct cost-effectiveness analyses, which generally measure HRQL in quality adjusted life-years (QALYs). The most commonly used utility-based scales include the Standard Gamble (Torrance, 1986), the Time Trade Off (Torrance, 1986), and the Feeling Thermometer (Torrance, 1986). Instruments that measure the value that society places on various health states include the Quality of WellBeing (QWB) scale (Kaplan et al, 1989), the Multiattribute Health Utilities Index (Feeny et al, 1992), and the EuroQol (The EuroQol Group, 1990). Most of the above instruments have been used in their generic form for a long time, however recently, the Standard Gamble and the Feeling Thermometer have been modified for use in children with asthma, and appear to show better measurement properties.

Evidence suggests that thus far both the SF-36 and the SIP have satisfactory properties for measuring HRQL as discriminative instruments in asthma. The SIP has shown good reliability (ICC=0.81) (Rutten van Molken, 1994), and acceptable crosssectional correlations with clinical indices of asthma and disease specific HRQL (Juniper 
et al, 1993, Rowe and Oxman, 1993, Rutten van Molken, 1994). The SF-36 has shown moderate cross-sectional correlations with clinical asthma severity in 5 of 9 domains, and with FEV1\% predicted in 3 of 9 domains (Bousquet et al, 1994). Two asthma clinical trials failed to show differences in treatment groups using the SIP (Rutten van Molken et al, 1994 and Jones, 1994). Also, the SIP was shown to have poor responsiveness in validation studies of disease-specific instruments (Juniper et al, 1993 and Marks et al, 1993).

The most commonly used disease-specific instruments in chronic pulmonary disease include Juniper's Asthma Quality of Life Questionnaire (J-AQLQ) (Juniper et al, 1993 and Juniper et al, 1992), Marks’ Asthma Quality of Life Questionnaire (M-AQLQ) (Marks et al, 1992, and Marks et al, 1993), the Living with Asthma Questionnaire (LWAQ) (Hyland, 1991), Life Activities Questionnaire for Asthma (LAQ) (Creer et al, 1992), Asthma Bother Profile (Hylan et al, 1995), St. George's Respiratory Questionnaire (SGRQ) (Jones et al, 1992), Respiratory Illness Quality of Life Questionnaire (QOLRIQ) (Maille et al, 1994 and Ferrer et al, 2002), the Chronic Respiratory Questionnaire (CRQ) (Guyatt et al, 1987), and the Functional Performance Inventory (FPI) (Leidy, 1994). Other less commonly used instruments include the How Are You (HAY) Questionnaire (le Coq et al, 2000), the Airways Questionnaire (AQ20/AQ30) (Alemayehu et al, 2002), and the ITG Asthma Short Form (Eisner et al, 2002). The first 5 instruments are asthma-specific, the SGRQ, the CRQ and the QOL-RIQ have been created for HRQL measurement in both asthma as well as COPD. The FPI has been created for measuring the functional performance component of HRQL in COPD only. Following is a review of the more commonly used instruments. These are grouped into 
two categories: 1) Instruments used in both asthma as well as COPD, and 2) Instruments used in either asthma only or in COPD only.

\section{Instruments Used in Both Asthma and COPD}

SGRQ: The SGRQ is a disease-specific questionnaire designed to measure the impact of COPD on overall health, daily life, and perceived well-being (Jones et al, 1992). This questionnaire contains 76 items covering the 3 domains of symptoms, activity and impact. The symptoms domain measures the frequency and severity of symptoms; the activities domain measures activities that cause or are limited by breathlessness; and the impacts domain measures the impact of COPD in terms of social functioning or psychological disturbances arising from chronic airways disease. The questionnaire takes around 10 minutes to complete, and can be self-administered or interviewer-administered (face-to-face or telephone). In Section I (symptoms domain), scaling of responses is on a 5-point Likert scale, whereas Sections II and III, which measure activity and impact respectively, are scored on a dichotomous Yes/No scale. There is a score for each section and a total summary score, each ranging from 0 to 100 , where 0 indicates best HRQL, and 100 indicates the worst HRQL.

The SGRQ has exhibited good psychometric properties. There is evidence of both test-retest reliability as well as internal consistency (Jones et al, 1991, and Jones et al, 1992). The instrument has also shown good evidence of validity. There was a high correlation between total scores and the presence of cough sputum and wheeze (Jones et al, 1992). Significant correlations have also been found between the symptoms, activity, and impact domains and other measures of disease activity such as FEV1, 6-minute 
walking distance (6-MWD), the Medical Research Council (MRC) respiratory symptoms questionnaire, MRC dyspnea grade, anxiety score, SIP total score, SIP symptoms domain, and the SIP psychosocial domain (Jones et al, 1992). The instrument has also been shown to be responsive (Jones et al, 1994). Based on empirical data and interviews with patients, a mean change in total score of 4 units is associated with slightly efficacious treatment, 8 units for moderately efficacious, and 12 units was associated with highly efficacious treatment (Jones et al, 1992 and Jones et al, 1994). This instrument has also been validated for its use in bronchiectasis (Wilson et al, 1997). This instrument has been used in clinical trials (Jones et al, 1994, and Lahdensuo et al, 1996). The MID for this instrument is 4 units on the total summary score (Jones et al, 1992).

Although the instrument was originally created and validated in the UK English language, it has also been translated into American English and has been found to be psychometrically stable (Barr et al, 2000). In addition to American English, this instrument has been translated into Finnish, Dutch, Italian, Spanish, French, Portuguese, Danish, Swedish, Polish and Chinese. Overall, the SGRQ has been widely recognized as having very good reliability and responsiveness in both COPD as well as asthma (Janssens, 2001).

CRQ: This instrument is a 20 -item questionnaire covering the 4 domains of dyspnea, fatigue, emotional function, and mastery (Guyatt et al, 1987). The questionnaire takes around 25-30 minutes to complete (which indicates high respondent burden), and each question has to be answered on a 7-point scale. The instrument has been shown to be reliable and responsive (Janssens, 2001). However, the biggest shortcoming of this 
instrument is that the questions are individualized. Therefore CRQ scores have to be compared pre- and post- intervention in the same patient. Hence, comparisons between groups of patients are theoretically invalid.

QOL-RIQ: This instrument consists of 55 questions covering the 7 domains of: breathing problems, physical problems, emotions, daily or domestic activities, social activities, relationships and sexuality, and general activities (Maille et al, 1994). The QOL-RIQ did show good correlations with clinical indices as well as measures of workplace productivity. However, the reliability (in terms of the intra-class coefficient) has not been established. The measurement properties for use of this instrument in clinical trials have not been established either. Like the St. Georges Respiratory Questionnaire, this instrument has been created to measure HRQL in asthma as well as COPD.

\section{Instruments Used in Either Asthma / COPD}

J-AQLQ: The J-AQLQ is a 32-item questionnaire, where items were chosen exclusively based on their importance to patients with asthma. Responses to each item were rated on a 7-point scale. The items are grouped into four domains such as symptoms, emotions, exposure to environmental stimuli, and activity limitations. The instrument is available in both self- as well as interviewer-administered format, and takes approximately 5 to 10 minutes to complete. The J-AQLQ has been validated by the creators (Juniper et al, 1993), validated in a clinical trial in an emergency room setting (Rowe and Oxman, 1993), and also validated in a study comparing salbutamol versus salmeterol clinical trial (von Molken et al, 1994), where measurement properties of various HRQL instruments 
were compared. The J-AQLQ demonstrated good reliability (ICCs ranging between 0.89 to 0.94 ), and very good responsiveness. The instrument showed cross-sectional and longitudinal correlations with clinical asthma measures as well as generic HRQL instruments as hypothesized.

M-AQLQ: The M-AQLQ is a 20-item, self-administered questionnaire consisting of the following 4 domains: breathlessness and physical restrictions, mood disturbance, social disruption and concerns for public health. Each item is rated on a 5-point scale. The instrument shows good reliability $(\mathrm{ICC}=0.80)$ (Marks et al, 1992). The instrument exhibits good discriminative properties (Sen et al, 1993). Sen and colleagues (1993) have also stated that although the M-AQLQ was primarily designed as an evaluative instrument, its psychometric characteristics make it appropriate for use as a discriminative instrument.

LWAQ: The LWAQ is a 68-item questionnaire covering 11 domains (social/leisure, sport, holidays, sleep, work, colds, morbidity, effects on others, medication use, sex and dysphoric states and attitudes). Patients rate their responses on a 3-point scale, thereby implying that the instrument may have poor responsiveness. This instrument shows very good discriminative properties, but poor evaluative properties (Hyland, 1991 and Juniper, 1995).

FPI: This instrument is a self-reported scale used to assess functional performance in COPD patients (Leidy, 1994). Performance is assessed in terms of day-to-day activities 
COPD patients need to do in order to meet basic needs. The instrument consists of 65 items over 6 domains (body care, household maintenance, physical exercise, recreation, spiritual activities and social activities). Responses are measured on a 4-point scale (from activity can be performed easily to activity could no longer be performed due to health reasons). The instrument has shown good evidence of reliability (Leidy, 1999) and validity (Leidy and Haase, 1996, Larson et al, 1998, Leidy, 1999).

For HRQL measurement in asthma, only the J-AQLQ and the M-AQLQ possess discriminative as well as evaluative properties. There is consistent evidence of responsiveness and longitudinal construct validity. In addition, the J-AQLQ quantifies the interpretation of the data in terms of clinical significance (a difference in 0.5 units per question can be termed as clinically significant). The QOL-RIQ has shown evidence of reliability but the evidence regarding its validity is ambiguous. The FPI has shown good reliability and validity, however, it can only be used to assess HRQL in COPD. The SGRQ is the only instrument that has demonstrated good evidence of psychometric stability in both asthma as well as COPD.

This chapter presented a review of the literature with respect to the clinical definition of asthma and COPD, their epidemiology and burden, pharmacotherapy and its effectiveness. The chapter also examined factors affecting nonadherence, the prevalence of nonadherence, and the different ways of assessing adherence. Finally, the chapter examined HRQL in asthma and COPD, in terms of its need and measurement issues followed by a discussion of various instruments used for HRQL measurement. The next chapter will discuss the methodology employed to achieve the goals of this study. 


\section{CHAPTER THREE}

\section{METHODOLOGY}

The purpose of this study was to examine the consequences of nonadherence to prophylactic pharmacotherapy in recipients with asthma and COPD. More specifically, this study has two goals. The first goal is to assess the impact of nonadherence on healthcare utilization and costs. The second goal is to assess the impact of nonadherence on HRQL. Phase II will also examine the impact of medication nonadherence on workplace productivity. This chapter will discuss in detail the methodologies used to achieve both goals.

\section{Phase I}

The study design, data source, study population and perspective, cohort definition, and patient inclusion and exclusion criteria are as follows.

\section{$\underline{\text { Data Source }}$}

The data source for this study consisted of the West Virginia Public Employees Insurance Agency (PEIA) data. The state of West Virginia provides healthcare benefits to its employees through the PEIA system. PEIA contracts with the pharmacy benefit management company Express Scripts Inc. to manage its pharmacy benefits. The state also contracts with Acordia Inc. to manage its medical claims. The PEIA administrative claims database consists of information on all aspects of member healthcare utilization, including pharmacy and medical claims. This database enables researchers to view a 
specific diagnosis, monitor the consequences of specific treatments or diseases, or even evaluate patterns of drug use across patients over time, as well as cross-sectionally. One can also examine the charges associated with a disease and its treatments. Specific fields included in the pharmacy and medical files are listed in Appendix A.

The recipient information includes patient name, social security number, birthdate, as well as demographic information such as address, ethnicity, marital status, and gender. The provider files consist of provider name and complete address, as well as provider specialty. For each claim, the data includes benefit year, the first and last date of service, date the claim was entered, date of admission, date of discharge, date the claim was received, claim adjustment date and the date of final processing. The billing information consists of amount that was charged, the paid amount, and deductibles or coinsurance, any out-of-pockets costs, as well as any amounts paid by Medicare. Medical claims also include a place of service field, which indicates whether the service took place in an inpatient or outpatient setting, physician office, urgent care center, surgical center, extended care facility, dental office or a birthing center. Medical claims also include Current Procedure Terminology -4 (CPT) procedure codes, as well as International Classification of Diseases $-9^{\text {th }}$ Edition, Clinical Modification (ICD-9-CM) diagnosis codes. There is one primary diagnosis code and up to nine secondary diagnosis codes. Thus, each claim can have up to ten ICD-9-CM codes listed. The pharmacy records include information regarding the date the prescription was written, the service date, days supply, quantity, whether the prescription was new or a refill, NDC drug code, drug name, drug category code, as well as dosage description. The billing information includes ingredient cost, dispensing fee, tax, copayment if any, and billed amount. In 
general, the amount paid by PEIA is the billed amount minus the copayment. This amount was used to assess pharmacy costs to PEIA. The pharmacy data also includes pharmacy related information such as pharmacy name, pharmacy number, and location.

\section{$\underline{\text { Study Population }}$}

The study population consisted of all PEIA recipients between 18 and 64 years of age, who had a diagnosis for chronic pulmonary ailments such as asthma, chronic obstructive pulmonary disease, chronic bronchitis, chronic airway obstruction or emphysema, and who were continuously enrolled for a 2-year period from July $1^{\text {st }}, 2001$ to June $30^{\text {th }}, 2003$. All patients with a primary diagnosis (ICD9) code for asthma (493.xx), or COPD (466.xx, 490.xx, 491.xx, 492.xx, 496.xx) in their medical claims were selected for this study.

\section{$\underline{\text { Data Extraction }}$}

The West Virginia PEIA medical and pharmacy claims data obtained from Accordia and Express Scripts, respectively, is loaded on the server maintained by West Virginia University's Accessible Intelligent Medication Strategies (AIMS) program, a grant funded academic detailing initiative. After obtaining approval from West Virginia PEIA and subsequently, West Virginia University Institutional Review Board (IRB) for conducting this study, the data needed for this study was extracted using the software BRIO-Intelligence. These data files were extracted as text files, and then converted into either Statistical Package for Social Sciences (SPSS) or MS Access datasets. Study subjects were identified using the specific inclusion and exclusion criteria, and the 
variables/fields that were relevant to this study were downloaded for the defined study period.

\section{Data Cleaning and Modification}

The data obtained after initial extraction from the AIMS server was divided into two files - medical and pharmacy. The medical claims files included claims for hospitalization, ED claims, and outpatient claims (physician visits). Elaborate criteria were used to separate the three types of claims - hospitalizations, ED visits and outpatient visits. In addition to the coding used in the datasets, strict criteria were used to verify that a particular episode was actually what it was supposed to be, as explained below.

In order to separate the hospitalization claims from the ED claims, diagnosis related groups (DRG) and Current Procedure Terminology (CPT) codes were used along with the LOS. The specific algorithm used to separate these claims is outlined as follows:

- First, a LOS variable was created based on the first date of service and the last date of service (number of days between the first date and the last date)

- All claims having a LOS greater than or equal to 1 were separated from those having a $\operatorname{LOS}=0$ days.

- All claims having a LOS $>=2$ days, and having a DRG code greater than zero were separated and classified as hospitalization claims.

- Among claims with LOS = 0 days, and having a CPT code between 99281 99285 or revenue codes between 450 - 459 were separated and classified as ED claims. 
- The remaining claims having $\operatorname{LOS}=0$ days were separated and classified as outpatient claims. Among outpatient claims, claims related to lab tests and transportation claims were removed from the outpatient files.

- In addition, claims that were rejected by PEIA were omitted in order to prevent duplication. A rejected claim was followed up with a negative claim. Both the negative and positive claims were rejected from the database.

\section{Study Perspective}

The selection of the appropriate study perspective was vital since it determines the methodology used to estimate the costs and benefits associated with the outcomes in question. In this study, the perspective used was that of PEIA, and costs to PEIA were used to estimate the economic outcomes. If it can be shown that substantial dollars can be saved as a consequence of maximum adherence, it will provide beneficial information to PEIA in designing recipient-directed interventions to promote adherence, as well as in making coverage-related policy decisions.

\section{Patient Exclusion, Treatment Arms and Time Frame}

The study design will be a retrospective, longitudinal cohort study. The time frame of the study will be two years, and six months. Since the study objective was to follow patients taking prophylactic pharmacotherapy, and examine the impact of using prophylactic medications on healthcare utilization and costs, it was imperative to use at least 2 years of data. This is an optimal time period since it allows enough time to examine the effects of nonadherence to pharmacotherapy to result in specific outcomes. 
Demographic (age, gender), clinical (diagnoses), resource utilization (outpatient, inpatient, prescription and emergency room use) and economic variables (amount billed to PEIA for any type of medical service rendered) were used for achieving the first study objective. Various study indices were calculated for the above variables retrieved from the PEIA dataset.

The following steps were followed in selecting the patients eligible for this study. First, the patients had to be continuously enrolled for the duration of the study period. All recipients having medical claims with a primary ICD-9-CM code for asthma (493.xx), patients with additional bronchitis/bronchiolitis (ICD-9-CM 466.xx), unspecified bronchitis (ICD-9-CM 490.xx), chronic bronchitis (ICD-9-CM 491.xx), emphysema (ICD-9-CM 492.xx), and chronic airway obstruction (ICD-9-CM 496.xx) were included in the cohort. Claims not reimbursed by PEIA were excluded from the analyses. PEIA recipients with asthma (ICD9 493.xx), and PEIA recipients with COPD (chronic bronchitis ICD9 491.xx, emphysema ICD9 492.xx, and chronic airway obstruction ICD9 496.xx, additional bronchitis ICD9 466.xx, unspecified bronchitis ICD9 490.xx) were analyzed separately. Also, recipients having medical claims with a primary diagnosis of both asthma as well as COPD were analyzed separately. Since misclassification of diagnosis could potentially be a major concern, patients having no prescriptions for asthma or COPD- related medications will be compared with those having prescriptions in terms of their mean outpatient visits and asthma or COPD-related total costs. If total asthma or COPD-related costs, and mean number of outpatient visits are lower for patients without prescriptions, it could have three implications. First, the patients are less severe disease, and do not require medications. Second, they require 
medications but are not being prescribed according to guidelines. The third possibility is that they do not have asthma or COPD, and have been falsely classified as having the disease, most likely due to data coding errors.

\section{Working Definition of Medication Adherence}

In order to assess adherence, only the use of maintenance medications as prophylactic therapy were included. These medications included inhaled corticosteroids, nedocromil, and cromolyn, long-acting $\beta$-agonists, and leukotrine modifiers. Using refill patterns from claims data, adherence indices were calculated to measure adherence. Although the supplement to the 1997 NHLBI Expert Panel 2 guidelines for asthma therapy recommend ICS as the preferred long-term control medication for asthma, many patients preferentially use nonsteroidal medications as prophylactic therapy, mainly due to the long-term adverse effects of taking steroids. Therefore, in the context of this study, medication adherence referred to obtaining regular refills of any medications in the therapeutic classes mentioned earlier. A comprehensive list of medications along with their therapeutic class is mentioned in Appendix C.

\section{Measurement Indices for Adherence}

The indices used to measure medication adherence are the medication possession ratio (MPR) and gaps in therapy. Both indices are described as follows.

\section{Medication Possession Ratio}

The MPR is defined as the sum of the days supply for all claims during a defined period of time divided by the number of days elapsed during the time period (Motheral 
and Fairman, 2000). The MPR yields a value between 0 and 1 . The MPR is affected by gaps in treatment, or terminations of treatment. Thus, the MPR does not take into account number of days the patient may have been hospitalized, in which medication use is not recorded. Recipients having no prescription claims were assigned a value of ' 0 ' for MPR. MPR was used as a continuous variable in the models, where 0 indicates $0 \%$ adherence, and 1 indicates $100 \%$ adherence.

\section{Gap Estimation}

A gap was defined as the number of days between the assumed depletion date of one claim (claims fill date plus days supply) and the fill date of the next refill (Motheral and Fairman, 2000). Median gaps were calculated for each recipient. Recipients having no prescriptions in the study period were assigned a value of 910 days for their median gap, since the total duration of their study period was 2.5 years (910 days).

\section{Demographic Variables}

The demographic variables used in the analyses were age and gender. Age was calculated by subtracting the birth-date from the index date of the study, which was July $1^{\text {st }}, 2001$. The field "recipient gender" was used to identify the patients' gender.

\section{Measurement of Medication-Related Variables}

Since the availability of drugs being used is limited, summary indices were calculated for medication-related variables. The Chronic Med Score (CMS) and Total Med Score (TMS) were calculated, which measured the total number of chronic medications and the total number of medications throughout the year, respectively (Balkrishnan and Christensen, 2001). CMS was defined as the number of medications 
for which a patient has four or more refills each year. The TMS was defined as the total number of medications for which the patient has had claims throughout the year. The Other Med Score would be $=$ TMS-CMS. Both TMS and CMS were used as continuous variables in the models.

\section{Index for Measuring Asthma Disease Severity}

Since the dataset did not provide information on disease severity, PEIA recipients with asthma were stratified into five severity levels based on their prescription drug use. This criterion has been successfully used to predict adverse outcomes such as hospitalizations (Grana et al, 1997) in asthma. The recipients will be classified into five severity levels (level I being least severe, and level 5 being most severe). Those PEIA recipients with asthma having three or less $\beta$-agonist claims in one year were classified as Level I. Those with four or more $\beta$-agonist claims without other asthma medications in one year were classified as Level II. Recipients in Level III consisted of individuals having a $\beta$-agonist claim, and at least one other asthma medication during one year. Level IV recipients consisted of individuals who had a claim for an oral steroid, and either a $\beta$-agonist or at least one other asthma medication, if they did not qualify for level V. Recipients classified as severity level V consisted of those with at least four or more B-agonists and at least one other asthma medication, as well as oral steroids dispensed more than two times in one year. This criteria has been successfully used as a proxy for asthma severity to risk-stratify asthma patients (Grana et al, 1997). Asthma disease severity was used as a continuous variable in the ordinary least squares (OLS) and 
Poisson regression models, and was included as a categorical variable in the logistic regression models.

\section{$\underline{\text { Research Design }}$}

The research design used for this part of the study was a quasi-experimental, cross-sectional, longitudinal design. The study involved examining the outcomes among patients with asthma and COPD in conjunction with medication adherence crosssectionally. The patients were followed for a period of two years and six months. Additionally, since the study involved following a cohort of patients longitudinally to examine the presence of adverse outcomes, and then classifying them as cases and controls, it can be called a cohort study.

\section{Data Analysis}

The data analysis for this phase is presented by the research questions proposed. The statistical packages SPSS ${ }^{\circledR}$ and SAS ${ }^{\circledR}$ were used to conduct the data analyses. The data analysis plan for patients with asthma and those with COPD are described separately.

For Asthma: (For all asthma analyses, only recipients with a primary diagnosis for asthma were used)

Research Question 1: Estimating whether medication prescribing was according to NHLBI guidelines

In order to address the first research objective, which was to examine whether medication prescribing was in accordance with NHLBI guidelines, all prescription claims 
for the sample population selected (recipients with a primary diagnosis code for asthma only) after applying the selection criteria were used. The NDC codes and the drug names were used to assign recipients into one of the four groups (as described in objective 1). This enabled us to assess how many patients received $\beta$-agonist only, how many patients received combination therapy without inhaled anti-inflammatory medications, how many patients received a prescription for inhaled anti-inflammatory therapy, and how many patients received no prescriptions for asthma. Frequencies were calculated to identify proportions of patients in each group, and subanalyses by age group and gender will also be carried out.

Research Question 2: Estimating the overall prevalence of asthma, and examining the prevalence by select demographic factors

The second objective of the study was to measure the prevalence of asthma, and to describe patterns of distribution of select demographic factors such as age group and gender in patients with asthma. All PEIA recipients having medical claims for asthma were used to measure prevalence. Rates of prevalence of asthma by age group and gender were calculated. Descriptive statistics such as chi-squares, t-tests, and ANOVA were used to examine differences in prevalence by each factor. Prevalence per $10,000=10,000 *$ (Number of PEIA members with asthma / total \# PEIA members)

Similarly, prevalence was calculated by age group, and gender with the denominator including the total number of PEIA members in each age group and gender, respectively. 


\section{Research Question 3: Estimating the prevalence of medication nonadherence}

The third study objective was to measure the prevalence of nonadherence to pharmacotherapy. Recipients having at least one claim for any medication listed as a prophylactic medication in the two-year study period were selected. A list of the medications included along with the therapeutic class is included in Appendix C. Based on the refill patterns for maintenance medications for these recipients, the adherence indices of MPR and median gap were calculated. Since the indices defined adherence differently, the use of both indices will enable us to validate the measurement of adherence and accurately assess its prevalence. Recipients who did not receive any prophylactic medication were categorized as nonadherent, and were assigned a value of ' 0 ' for their MPR, and 910 days for their median gap.

Research Question 4: Estimating the impact of medication adherence on the number of hospitalizations, ED visits, outpatient visits, and LOS

The fourth objective was to assess the association between medication nonadherence and healthcare utilization as indicated by the number of hospitalizations, ED visits, outpatient visits, and inpatient LOS. For the dependent variables of adverse outcomes (hospitalizations and/or ED visits), and number of outpatient visits, the use of OLS regression would be inappropriate. This is because when the dependent variable is count data, very few patients have a value greater than 0 , thereby violating the assumption of normal distribution. However, OLS regression has been used when the dependent variable is count data in health services research (Buzcko, 1989, and Balkrishnan and Christensen, 2000). The use of OLS can be justified if the number of 
zeroes in the dependent variable are at a minimum. However, since a majority of asthma and COPD patients in this study had no hospitalizations or ED visits, a Poisson distribution was assumed. Thus, for the dependent variables of number of adverse outcomes, and number of outpatient visits, Poisson regression was used (Wang, 2003). This regression assumed that the dependent variable (count data) has a Poisson distribution. If the variance was greater than the mean, the Poisson regression procedure was replaced by the negative binomial regression procedure. OLS regression was used for the model having LOS as the dependent variable. For the OLS regression model, the assumptions of normality, multicollinearity, autocorrelation, and heteroskedasticity were checked. The Variation Inflation Factor (VIF) was used to check for multicollinearity (VIF $>10$ indicates multicollinearity). The Durbin-Watson Statistic was used to check for autocorrelation. For 5 or more independent variables, and more than 100 cases, a Durbin-Watson statistic between 1.57 and 2.22 indicates no autocorrelation. The model was also checked for heteroskedasticity by examining the graph of predicted values vs. residual values. Three different regression models were run, with the dependent variables being number of adverse outcomes (either hospitalizations or number of ED visits), number of outpatient visits, and LOS. In addition to nonadherence, all the independent variables used are shown in the model below. Separate regression models were run with MPR and median gap as primary predictor variables.

The model is represented as:

$$
\begin{aligned}
& \text { Utilization }=\beta 0 \\
& +\beta 1 \text { (Adherence) } \\
& +\beta 2 \text { (demographics }- \text { age, gender) }
\end{aligned}
$$




$$
\begin{aligned}
& +\beta 3 \text { (disease severity }- \text { based on prescription drug use) } \\
& +\beta 4 \text { (medication use }- \text { TMS, CMS) } \\
& + \text { Error }
\end{aligned}
$$

Where $\beta 1$ represented medication adherence as indicated by MPR or median gap.

$\beta 2$ represented demographic characteristics of age and gender. Age was included as a continuous variable, and gender was dichotomous variable, with females coded as 0 , and males coded as 1 .

$\beta 3$ indicated asthma disease severity, as indicated by the pharmaceutically determined index (Grana et al, 1997).

$\beta 4$ indicated the number of total medications (TMS) or the number of chronic medications (CMS) per year. Both TMS and CMS were included as continuous variables.

\section{Research Question 5: Estimating the impact of medication adherence on total costs}

The fifth objective was to assess the association between medication nonadherence and total healthcare costs (hospitalizations, ED, outpatient, and prescription costs). OLS regression analysis was used to achieve this objective. The dependent variable was log-transformed total costs. Costs were log-transformed in order to achieve normality. In addition, the VIF was used to examine multicollinearity, and the Durbin-Watson statistic was used to examine autocorrelation. The model was checked for heteroskedasticity by examining the graph of predicted values versus residual values. Separate regression models were run with MPR and median gap as primary predictor variables. 
The model is represented as:

$$
\begin{aligned}
\text { Total Costs }= & \beta 0 \\
& +\beta 1 \text { (Adherence) } \\
& +\beta 2(\text { demographics }- \text { age, gender }) \\
& +\beta 3(\text { disease severity }- \text { based on prescription drug use }) \\
& +\beta 4(\text { medication use }- \text { TMS }, \mathrm{CMS}) \\
& + \text { Error }
\end{aligned}
$$

Where $\beta 1$ represented medication adherence as indicated by MPR or median gap.

$\beta 2$ represented demographic characteristics of age and gender. Age was included as a continuous variable, and gender was dichotomous variable, with females coded as 0 , and males coded as 1 .

$\beta 3$ indicated asthma disease severity, as indicated by the pharmaceutically determined index (Grana et al, 1997).

$\beta 4$ indicated the number of total medications (TMS) or the number of chronic medications (CMS) per year. Both TMS and CMS were included as continuous variables. 


\section{Research Question 6: Estimating the relative risk of adverse outcomes}

The sixth objective was to examine the relative risk of adverse health outcomes such as a hospitalization or an ED visit as a consequence of medication nonadherence. In research question 4, the association between nonadherence and the number of hospitalizations and ED visits was examined. Although this helped us to assess how utilization changed with nonadherence, the presence of a temporal relationship is necessary to attribute any causal effects. In research question 4, adherent behavior was measured as an average for the study period. It was necessary to measure nonadherence in the time period preceding the event of interest. Through this research question, how nonadherence impacted the probability of an adverse outcome such as an ED visit or a hospitalization was assessed. The methodology used to achieve this objective was a casecontrol study, and is described as follows.

Case-Control Study Methodology: This objective measured the impact of nonadherence on the probability of an adverse outcome, comparing patients who experienced the event (cases), with those who did not (controls). All PEIA recipients with asthma (identified from medical claims) were followed from the $1^{\text {st }}$ to the $24^{\text {th }}$ month of the study period. Any recipient with an adverse outcome between the $7^{\text {th }}$ to the $24^{\text {th }}$ month in this time period was a case. The number of refills for any maintenance drug in the 6 months prior to the adverse outcome were determined to assess nonadherence. An MPR for these six months was calculated based on the number of refills. The controls consisted of recipients who had no adverse outcomes between the $7^{\text {th }}$ and $24^{\text {th }}$ months. For the controls, adherence was measured by the MPR during the first six months of the study 
period. A logistic regression model was used with a dichotomous dependent variable (indicating presence/absence of an adverse outcome).

The model is represented as:

$$
\begin{aligned}
& \text { Adverse outcome }=\beta 0 \\
& +\beta 1 \text { (Adherence - MPR for } 6 \text { months) } \\
& +\beta 2 \text { (demographics - age, gender) } \\
& +\beta 3 \text { (disease severity }- \text { based on prescription drug use) } \\
& +\beta 4 \text { (medication use }- \text { TMS, CMS) } \\
& + \text { Error }
\end{aligned}
$$

Where $\beta 1$ represented medication adherence as measured by the MPR for 6 months. $\beta 2$ represented demographic characteristics of age and gender. Age was included as a categorical variable, and gender was dichotomous variable, with females coded as 0 , and males coded as 1 .

$\beta 3$ indicated asthma disease severity, as indicated by the pharmaceutically determined index (Grana et al, 1997). This index was entered as a categorical variable, with Level I asthma patients as the reference group.

$\beta 4$ indicated the number of total medications (TMS) or the number of chronic medications (CMS) per year. Both TMS and CMS were included as continuous variables. 
For COPD: (For all COPD analyses, only recipients with a primary diagnosis for COPD only were used)

Research Question 1: Estimating whether medication prescribing was according to GOLD guidelines

In order to address the first research objective, which was to examine whether medication prescribing was in accordance with GOLD guidelines, all prescription claims for the sample population selected (recipients with a primary diagnosis code for COPD only) after applying the selection criteria were used. The NDC codes and the drug names were used to assign recipients into one of the three groups (as described in objective 1). This enabled the assessment of the number of patients who received bronchodilators (long and short-acting beta-agonists, anticholinergics, and methylxanthines), corticosteroids (oral as well as inhaled), or no pharmacotherapy. Frequencies were calculated to identify proportions of patients in each group, and subanalyses by age group and gender were carried out.

Research Question 2: Estimating the overall prevalence of COPD, and examining the prevalence by select demographic factors

The second objective of the study was to measure the prevalence of COPD, and to describe patterns of distribution of select demographic factors such as age group and gender in patients with COPD. All PEIA recipients having medical claims for COPD were used to measure prevalence. Rates of prevalence of COPD by age group, and 
gender were calculated. Descriptive statistics such as chi-squares, t-tests, and ANOVA were used to examine differences in prevalence by each factor.

Prevalence per $10,000=10,000 *$ (Number of PEIA members with COPD/Total \# PEIA members)

Similarly, prevalence was calculated by age group, and gender with the denominator including the total number of PEIA members in each age group and gender, respectively.

Research Question 3: Estimating the prevalence of medication nonadherence

The third study objective was to measure the prevalence of nonadherence to pharmacotherapy. Recipients having at least one claim for any medication listed as a prophylactic medication in the two-year study period were selected. Based on the refill patterns for maintenance medications for these recipients, the adherence indices of MPR and median gap were calculated. Recipients who did not receive any prophylactic medication were categorized as nonadherent, and were assigned a value of ' 0 ' for their MPR and 910 days for their median gap.

Research Question 4: Estimating the impact of medication adherence on the number of hospitalizations, ED visits, outpatient visits, and LOS

The fourth objective was to assess the association between medication nonadherence and healthcare utilization as indicated by the number of hospitalizations, ED visits, outpatient visits and LOS. For the dependent variables of numbers of ED visits, hospitalizations and number of outpatient visits, Poisson regression was used. If 
the variance was greater than the mean, the Poisson regression procedure was replaced by the negative binomial regression procedure. OLS regression was used for the model having LOS as the dependent variable. For the OLS regression model, the assumptions of normality, multicollinearity, autocorrelation, and heteroskedasticity were checked. The VIF was used to examine multicollinearity, and the Durbin-Watson statistic was used to examine autocorrelation. The model was checked for heteroskedasticity by examining the graph of predicted values versus residual values. Three different regression models were run, with the dependent variables being number of adverse outcomes (either hospitalizations and/or number of ED visits), number of outpatient visits, and LOS. In addition to nonadherence, all the independent variables used are shown in the model below. Separate regression models were run with MPR and median gap as predictor variables.

The model is represented as:

$$
\begin{aligned}
& \text { Utilization }=\beta 0 \\
& +\beta 1 \text { (Adherence) } \\
& +\beta 2 \text { (demographics }- \text { age, gender) } \\
& +\beta 3 \text { (medication use }- \text { TMS, CMS) } \\
& + \text { Error }
\end{aligned}
$$

Where $\beta 1$ represented medication adherence as indicated by MPR or median gap. $\beta 2$ represented demographic characteristics of age and gender. Age was included as a continuous variable, and gender was dichotomous variable, with females coded as 0 , and males coded as 1 . 
$\beta 3$ indicated the number of total medications (TMS) or the number of chronic medications (CMS) per year. Both TMS and CMS were included as continuous variables.

Research Question 5: Estimating the impact of medication adherence on total costs

The fifth objective was to assess the association between medication nonadherence and total healthcare costs (hospitalizations, ED, outpatient, and prescription costs). OLS regression analysis was used to achieve this objective. The dependent variable was log-transformed total costs. Costs were log-transformed in order to achieve normality. In addition, the VIF was used to examine multicollinearity, and the Durbin-Watson statistic was used to examine autocorrelation. The model was checked for heteroskedasticity by examining the graph of predicted values versus residual values. Separate regression models were run with MPR and median gap as primary predictor variables.

The model is represented as:

$$
\begin{aligned}
\text { Total Costs }= & \beta 0 \\
& +\beta 1(\text { Adherence }) \\
& +\beta 2(\text { demographics }- \text { age }, \text { gender }) \\
& +\beta 3(\text { medication use }- \text { TMS }, \text { CMS }) \\
& + \text { Error }
\end{aligned}
$$

Where $\beta 1$ represented medication adherence as indicated by MPR or median gap. 
$\beta 2$ represented demographic characteristics of age and gender. Age was included as a continuous variable, and gender was dichotomous variable, with females coded as 0 , and males coded as 1 .

$\beta 3$ indicated the number of total medications (TMS) or the number of chronic medications (CMS) per year. Both TMS and CMS were included as continuous variables.

Research Question 6: Estimating the relative risk of adverse outcomes

The sixth objective was to examine the relative risk of adverse health outcomes such as a hospitalization or an ED visit as a consequence of medication nonadherence. In research question 4, we examined the association between nonadherence and the number of hospitalizations and ED visits. Although this helped assess how utilization changed with nonadherence, the presence of a temporal relationship is necessary to attribute any causal effects. In research question 4, adherent behavior was measured as an average for the study period. Nonadherence was measured in the time period preceding the event of interest. This research question (question 6) assessed how nonadherence impacted the probability of an adverse outcome such as an ED visit or a hospitalization. The methodology used to achieve this objective was a case-control study, and is described as follows.

Case-Control Study Methodology: This objective measured the impact of nonadherence on the probability of an adverse outcome, comparing patients who experienced the event (cases), with those who did not (controls). All PEIA recipients with COPD (identified from medical claims) were followed from the $1^{\text {st }}$ to the $24^{\text {th }}$ month of the study period. 
Any recipient with an adverse outcome between the $7^{\text {th }}$ to the $24^{\text {th }}$ month in this time period was a case. The number of refills for any maintenance drug in the six months prior to the adverse outcome was determined to assess nonadherence. An MPR for these six months was calculated based on the number of refills. The controls consisted of recipients who had no adverse outcomes between the $7^{\text {th }}$ and $24^{\text {th }}$ months. For the controls, adherence was measured by the MPR for the first six months of the study period. A logistic regression model was used with a dichotomous dependent variable (indicating presence/absence of an adverse outcome).

The model is represented as:

$$
\begin{aligned}
& \text { Adverse outcome }=\beta 0 \\
& +\beta 1 \text { (Adherence - MPR for six months) } \\
& +\beta 2 \text { (demographics - age, gender) } \\
& +\beta 3 \text { (medication use - TMS, CMS) } \\
& + \text { Error }
\end{aligned}
$$

Where $\beta 1$ represented medication adherence as indicated by the MPR for six months. $\beta 2$ represented demographic characteristics of age and gender. Age was included as a categorical variable, and gender was dichotomous variable, with females coded as 0 , and males coded as 1 .

$\beta 3$ indicated the number of total medications (TMS) or the number of chronic medications (CMS) per year. Both TMS and CMS were included as continuous variables. 


\section{Phase II}

The primary objective of the second phase was to examine the relationship between nonadherence to ICS pharmacotherapy, and HRQL. In order to achieve this objective, primary data was collected. After obtaining permission from the West Virginia University IRB, a questionnaire was mailed to patients with asthma and COPD, which contained the SGRQ, the Morisky adherence scale, and questions to measure losses in workplace productivity. Nonadherence was assessed using secondary data from Phase I, as well as self-reported nonadherence from the Morisky scale. The following section describes the methodology used to identify the study population, rationale for choosing the survey instrument, data collection process and the statistical techniques used in Phase II.

\section{$\underline{\text { Study Population }}$}

The study population for Phase II consisted of patients with asthma and COPD who were selected based on the inclusion/exclusion criteria used for Phase I selection. Thus, adult recipients (between 18 and 64 years of age) who had a claim for asthma or COPD between the duration of the study period July $1^{\text {st }}, 2001$ to June $30^{\text {th }}, 2003$ were selected. The recipients were continuously enrolled in PEIA during this study period.

\section{Sampling Technique}

Based on the above inclusion criteria, 1,493 recipients had diagnosis codes for asthma only, whereas 7,161 recipients had primary diagnoses of COPD only, and 940 recipients had diagnosis codes for both asthma and COPD, in their medical claims. The 
total number of recipients with asthma and / or COPD was 9,594. All patients with asthma only $(n=1,493)$, and both asthma and COPD $(n=940)$ were mailed the HRQL questionnaire. For recipients with COPD only, a random sample of 2,000 recipients was selected from the 7,161 recipients, and were mailed the questionnaire. Simple Random Sampling (SRS) without replacement was used to identify the random sample of COPD recipients (Kalton $\mathrm{G}, 1987$ ). In this technique, the randomly selected recipients in the sample are not replaced to be given a further chance of selection. SRS without replacement yields more precise estimates than sampling with replacement, hence this technique was used. Also, SRS technique allows the use of both means as well as proportions along with the associated confidence intervals.

\section{Sample Size Determination for COPD-only Recipients}

Among recipients with primary diagnosis codes for COPD-only $(n=7,161), 2000$ randomly selected recipients were mailed the HRQL questionnaire. The following formula was used for calculating the SRS for this study sample:

$$
\mathrm{n}=(\mathrm{z} / \mathrm{e}) 2 * \pi(1-\pi)
$$

Where,

$\mathrm{n}$ is the sample size

$\mathrm{z}$ is the number of standard errors for a given confidence interval

$\pi$ is the estimated proportion of people who were adherent to their medication

$\mathrm{e}$ is the required estimator 
Based on the table of normal distribution, (95\% of normal distribution falls within 1.96 standard deviations around the distribution's means), for confidence interval of $95 \%, z=1.96$. An estimator that is within $5 \%$ of the population percentage with $95 \%$ probability, was employed in the study (Kalton G, 1987).

It has been shown that adherence rates among chronic pulmonary disease patients range between 3-48\% (Fish and Lung 2001, Bender 2002). Thus, $\pi$ could be anywhere between 0.05 and 0.48 . However, the most conservative sample size would be obtained by taking $\pi=0.5$, hence 0.5 was chosen as the value of $\pi$.

Inputting above values in the equation yielded $\mathrm{N}=384$.

Net sample size $=($ Gross Sample Size $) *($ Mail Response Rate $) *($ Usable Response Rate $)$

Hence, Gross Sample Size $=$ Net Sample Size $/($ Mail Response Rate $) *($ Usable Response Rate)

We assumed a conservative response rate of $20 \%$, and that only $90 \%$ of the responses would be usable. Hence,

Gross Sample Size $=384 / 0.20 * 0.9=2000$

Thus, a total of 2,000 recipients with COPD were surveyed.

\section{Health-Related Quality of Life Assessment}

The St. George's Respiratory Questionnaire (SGRQ) was used to measure HRQL in patients with asthma and COPD (Jones et al, 1992). This instrument has been shown 
to be a valid predictor of HRQL in patients with asthma as well as COPD (Jones et al, 1992, Barr et al, 2000). PEIA recipients having medical claims with primary ICD9 codes for asthma and COPD were mailed the SGRQ to measure their HRQL. This questionnaire, developed by Jones and colleagues (1992) has also been validated in the US population (Barr et al, 2000). The SGRQ is a 76-item questionnaire, grouped into three domains (symptoms, activity, and impacts). Responses in the symptoms domain were rated on a 7-point scale, whereas the activity and impacts domains consisted of dichotomous (yes/no) responses. Each domain score as well as the total HRQL score is on a scale of 0 to 100 , where 0 indicates best HRQL, and 100 indicates worst HRQL. Also, a difference in total HRQL score of 4 units is considered as a clinically significant difference.

For the purpose of this study, the SGRQ will be used as a discriminative instrument. This is because the objective of Phase II is to examine differences between two groups of patients: those who were adherent, and those who were nonadherent to ICS pharmacotherapy. Instruments used to distinguish between two or more groups of patients are discriminative in nature. The psychometric properties required of a discriminative instrument are reliability and cross-sectional construct validity. This instrument has shown good evidence of reliability as well as validity in a US population (Barr et al, 2000).

\section{Self-Reported Nonadherence Assessment}

In addition to the HRQL questionnaire, the Morisky adherence scale (Morisky et al, 1986 and Pratt et al, 2001), and the Work Productivity Short Inventory (WPSI) also 
known as the Wellness Inventory (Goetsel et al, 2003), were added to the SGRQ. The Morisky Scale, which is a 4-item questionnaire, used to measure self-reported adherence has been used in recipients with various disease conditions (Morisky et al, 1986 and Pratt et al, 2001). Scores obtained from this scale range from 0 to 4 , where 0 indicates high adherence, and 4 indicates nonadherence. Patients responding with a score of ' 0 ' are classified as 'high adherent', patients having scores from 1-2 were classified as 'medium adherent', and patients having scores greater than 2 were classified as 'low adherent.' (Morisky et al, 1988).

\section{Workplace Productivity Assessment}

In addition to the adherence measure, the WPSI or Wellness Inventory was used to measure losses in workplace productivity due to both asthma and COPD (Goetsel et al, 2003). This instrument consists of 4 questions measuring the number of days the disease was experienced, the number of days the patient was absent due to the disease, and the number of hours the patient was unproductive while experiencing the disease. The number of days absent, as well as the number of unproductive hours were translated into dollar metrics. The formulae for calculating absenteeism costs (losses in productivity when absent from work due to disease), presenteeism costs (losses in productivity when present at work due to disease), and total costs are shown below. The average hourly wage rate for West Virginia for 2002 was $\$ 14.04$ (US Dept. of Labor, 2003). For calculating presenteeism costs, the number of days experienced was multiplied by 236.5/365 to adjust for the number of workdays in a year (Goetsel et al, 2003). 


$$
\begin{aligned}
\text { Absenteeism Costs }= & \text { Total Days Absent } * 8 \text { Hours * \$14.04 } \\
\text { Presenteeism Costs }= & (\text { Days Experienced } * 236.5 / 365)-\text { Days Absent * } \\
& \# \text { Unproductive Hours * } 14.04
\end{aligned}
$$

Total Costs $=$ Absenteeism costs + Presenteeism costs

\section{$\underline{\text { Data Collection }}$}

PEIA recipients identified with asthma and COPD from the medical claims were mailed the questionnaire (consisting of the SQRQ, the Morisky scale, and the WPSI) along with a personalized cover letter and a business reply envelope. The cover letter explained the purpose of the survey, which would be to assess the HRQL among recipients with asthma or COPD, and assured confidentiality of the respondents.

Data from the survey respondents was linked to their adherence scores obtained from the claims data. HRQL scores for each domain, as well as total summary scores were compared between respondents with good adherence and those with poor adherence. HRQL scores were also compared with prior healthcare utilization in order to examine the overall relationship between nonadherence, utilization of healthcare services, and HRQL. Since the questionnaire was mailed in October 2003, medical and pharmacy claims were extracted for 6 additional months for the study population - from July through December 2003. This allowed the examination of utilization behavior at the time of measuring HRQL. 


\section{Data Entry and Analysis}

Each questionnaire received was checked for completeness and clinical relevance. Respondents who had entered less than $76 \%$ of the items in the questionnaire were omitted from the analysis, as recommended by Jones and colleagues (1992). Data from all questionnaires considered acceptable was entered into an $\operatorname{SPSS}^{\circledR}$ dataset. The analysis plan for examining each research question in Phase II is explained as follows.

Research Question 7: To examine the HRQL of PEIA patients with asthma and COPD

This research question consisted of examining domain-specific as well as total summary HRQL scores for the asthma-only, COPD-only, and both groups. HRQL scores among PEIA patients with asthma and COPD were compared with those from other studies that used the SGRQ. T-tests and one-way ANOVAs were used to compare HRQL scores among the three groups.

Research Question 8: To examine the self-reported nonadherence of PEIA recipients with asthma and COPD

Based on the Morisky scale scores, respondents were classified as high, medium, or low adherent. The prevalence of adherence was compared among the three groups (asthma-only, COPD-only, and both) by using chi-square statistics. 
Research Question 9: To examine the congruence between self-reported and claimsbased medication adherence

This research question examines the congruence between adherence measured using claims data and self-reported adherence from the Morisky scale. Spearman's correlation was used to measure the correlation between the two adherence scores for the survey respondents. In order to account for the possibility of misclassification, which may result in patients not having any prescriptions in their medical claims, only respondents having at least one prescription for maintenance medications (MPR $>0$ ) were used for the purpose of addressing this research question. Spearman's correlation between refill-based adherence measures and the Morisky scale score was calculated.

Research Question 10: To examine differences in HRQL by medication nonadherence

This question examines differences in HRQL scores between adherent and nonadherent respondents. Bivariate analyses were carried out to examine differences in domain-specific as well as total summary HRQL scores among respondents who were classified as high adherent, medium adherent, and low adherent based on scores obtained from the Morisky scale, and mean MPR and median gap scores were compared for each of the three groups - asthma-only, COPD-only, and both. Chi-square statistics and oneway ANOVAs were used for this analysis. In order to measure the impact of claims-data based adherence on HRQL, OLS regression analyses were carried out with the total summary score as the dependent variable. Separate models were run for each of the asthma-only, COPD-only, and both groups. Also separate models were run with MPR adherence and Morisky scale adherence as predictor variables. Morisky scale was used 
as a categorical variable, with respondents classified as high adherent as the reference group. MPR was used as a continuous variable on a scale from 0 to 1 . Age, TMS, and CMS were used as continuous variables, whereas gender was used as a dichotomous variable, with females coded as 0 , and males coded as 1 .

The model is represented as:

$$
\begin{aligned}
\text { Total HRQL } & =\beta 0 \\
& +\beta 1(\text { Adherence }- \text { MPR or Morisky) } \\
& +\beta 2(\text { demographics }- \text { age }, \text { gender }) \\
& +\beta 3(\text { medication use }- \text { TMS }, \text { CMS }) \\
& + \text { Error }
\end{aligned}
$$

Research Question 11: To examine the impact of medication adherence on workplace productivity

This question examines the impact of nonadherence on workplace productivity. Absenteeism, presenteeism, as well as total productivity costs were compared between the asthma-only, COPD-only, and both groups using t-tests and one-way ANOVAs. In order to assess the impact of medication nonadherence on productivity costs, OLS regression analysis was used with total productivity costs as the dependent variable. Separate regression models were run for each group - asthma-only, COPD-only, and both. Also separate models were run with MPR adherence and Morisky scale adherence as predictor variables. Morisky scale was used as a categorical variable, with respondents classified as high adherent as the reference group. MPR was used as a continuous variable on a scale from 0 to 1 . Age, TMS, and CMS were used as 
continuous variables, whereas gender was used as a dichotomous variable, with females coded as 0 , and males coded as 1 .

The model is represented as:

$$
\begin{aligned}
& \text { Total Productivity Costs }=\beta 0 \\
& +\beta 1 \text { (Adherence }- \text { MPR or Morisky) } \\
& +\beta 2 \text { (demographics - age, gender) } \\
& +\beta 3 \text { (medication use }- \text { TMS, CMS) } \\
& + \text { Error }
\end{aligned}
$$

This chapter has discussed in detail the methodology used to achieve both Phase I and Phase II objectives. The next chapter will consist of the results obtained from the analyses. 


\section{CHAPTER FOUR}

\section{RESULTS AND DISCUSSION}

Chapters I and II have thus far described the need for conducting this study, existing evidence regarding medication adherence and its impact on outcomes in respiratory illness, and study objectives. Chapter III described in detail the methodology used to achieve these objectives. The impact of medication adherence was measured using a retrospective analysis of WV PEIA claims data in Phase I, and HRQL, selfreported medication adherence, and losses in productivity were measured in Phase II using a mail survey. This chapter provides the results of the analyses along with a discussion of the results.

\section{Phase I Results}

Phase I of this study consisted of analyzing medical and pharmacy claims data of WV PEIA members with asthma and COPD from July 2001 through December 2003. The overall goal of this analysis was to determine the impact of medication nonadherence (as determined by refill patterns in claims data) on overall healthcare utilization and costs, and adverse outcomes such as ED visits and hospitalizations.

WV PEIA claims data were provided by Accordia (medical claims), and Express Scripts (pharmacy claims). On average, PEIA had around 119,725 adult enrollees per year in 2001, 2002, and 2003 in WV. Of these, around 16,000 enrollees had an ICD-9 code for asthma and/or COPD in their medical claims. Of these, only continuously eligible enrollees between the ages of 18 and 64 years were selected for the purpose of this study, which yielded 9,594 enrollees. Among these, 1,493 enrollees had primary 
ICD-9 codes for asthma only, 7,161 enrollees had ICD-9 codes for COPD-only, and 940 enrollees had ICD-9 codes for asthma as well as COPD. These enrollees were also nonmanaged care, and not Medicare eligible, since they were less than 65 years of age.

Pharmacy claims for these enrollees were extracted. Table 2 shows the overall extraction process from claims data. 
Table 2: Selection of Study Sample for Phase I

Inclusion Criteria

STEP 1: Pre-extraction

Total Adult PEIA Recipients in WV

STEP 2: Recipients with Asthma/COPD

Number of Recipients

STEP 3: Non-Medicare and Continuously Eligible

Between the Ages of $18-64$ years

Number of Recipients

Asthma-only

COPD-only

Both Asthma and COPD

$$
\mathrm{N}=16,000
$$

$$
\mathrm{N}=9,594
$$

$\mathrm{N}=1,493$

$$
\begin{aligned}
& 2001 \mathrm{~N}=112,150 \\
& 2002 \mathrm{~N}=121,082 \\
& 2003 \mathrm{~N}=125,942
\end{aligned}
$$

Number of Recipients

$$
\begin{gathered}
N=7,161 \\
N=940
\end{gathered}
$$


The demographic characteristics of the final Phase 1 study participants are shown in Table 3. For the overall Phase I sample, the average age was around $50 \pm 9.6$ (SD) years. This can be explained by the fact that the majority of the recipients were COPD patients, which is more prevalent in older individuals. Almost $70 \%$ of all adults were between 46 and 64 years of age. The sample was predominantly female, with only onethird of the sample recipients being male. The trend for age and gender distribution was similar for the asthma-only, COPD-only, and the both groups. There was a slightly higher percentage of $46-64$ year olds in the both group. Also, the proportion of females in the both group was higher than the COPD-only group. Data on race distribution was unavailable. 
Table 3: Demographic Characteristics of the Phase 1 Final Study Recipients

\begin{tabular}{|c|c|c|c|c|c|c|c|c|}
\hline $\begin{array}{l}\text { Demographic } \\
\text { Characteristics }\end{array}$ & \multicolumn{2}{|c|}{ Overall Sample } & \multicolumn{2}{|c|}{ Asthma-only } & \multicolumn{2}{|c|}{ COPD-only } & \multicolumn{2}{|c|}{$\begin{array}{l}\text { Both Asthma } \\
\text { and COPD }\end{array}$} \\
\hline Age* & $\mathrm{N}$ & $\%$ & $\mathrm{~N}$ & $\%$ & $\mathrm{~N}$ & $\%$ & $\mathrm{~N}$ & $\%$ \\
\hline $18-30$ & 435 & 4.5 & 86 & 5.8 & 322 & 4.5 & 27 & 2.9 \\
\hline $31-45$ & 2,475 & 25.8 & 417 & 27.9 & 1,862 & 26.0 & 196 & 20.9 \\
\hline $46-64$ & 6,684 & 69.7 & 990 & 66.3 & 4,977 & 69.5 & 717 & 76.3 \\
\hline $\begin{array}{l}\text { Mean Age } \pm \text { SD } \\
\text { Gender* }\end{array}$ & 49.5 & 9.6 & 48. & 9.9 & 49. & $=9.6$ & & \pm 8.9 \\
\hline Male & 3,386 & 35.3 & 422 & 28.3 & 2,719 & 38.0 & 245 & 26.1 \\
\hline Female & 6,208 & 64.7 & 1,071 & 71.7 & 4,442 & 62.0 & 695 & 73.9 \\
\hline
\end{tabular}

*Significantly different $(\mathrm{p}<=0.05)$; SD: Standard Deviation 
Overall healthcare utilization and costs for the overall sample as well as each of the 3 disease groups for the entire duration of the study (2.5 years) are shown in Table 4. For the overall Phase I sample, prescription costs were the largest cost driver (50.7\%), followed by outpatient costs (33.7\%). The same trend was observed for each of the three disease groups. Among the asthma-only group enrollees, hospitalization costs constituted $7.4 \%$ of total costs, outpatient costs constituted $35.5 \%$ of total costs, and prescription costs constituted more than half (55.7\%) of total costs. Among the COPD-only group enrollees, hospitalization costs constituted $14.4 \%$ of total costs, outpatient costs constituted $38.8 \%$ of total costs, and prescription costs constituted slightly less than half (47.5\%) of total costs. Among both-group enrollees, hospitalization costs as a percentage of total costs were higher than those for the asthma-only and the COPD-only groups (15.7\%), whereas outpatient costs as a percentage of total costs were lower than those for the asthma-only or COPD-only groups (29.4\%). ED costs as a percentage of total costs were slightly higher for the COPD-only and the both groups, as compared to the asthmaonly group. 
Table 4: Expenditures as a Percentage of Total Costs for the Entire Study Period

\begin{tabular}{|c|c|c|c|c|}
\hline Cost Type & $\begin{array}{l}\text { Overall } \\
\text { Sample }\end{array}$ & $\begin{array}{l}\text { Asthma-only } \\
\text { \$ (\% of Total }\end{array}$ & $\begin{array}{l}\text { COPD-only } \\
\text { \$ (\% of Total }\end{array}$ & $\begin{array}{c}\text { Both } \\
\text { \$ (\% of Total }\end{array}$ \\
\hline & $\begin{array}{c}\text { \$ (\% of Total } \\
\text { Costs })\end{array}$ & Costs) & Costs) & Costs) \\
\hline $\begin{array}{l}\text { Hospitalization } \\
\text { Costs }\end{array}$ & $\begin{array}{c}732,157 \\
(13.5)\end{array}$ & $\begin{array}{c}72,914 \\
(7.4)\end{array}$ & $\begin{array}{c}390,382 \\
(14.4)\end{array}$ & $\begin{array}{c}268,861 \\
(15.7)\end{array}$ \\
\hline ED Costs & $114,652(2.1)$ & $13,181(1.4)$ & $62,781(2.3)$ & $38,690(2.3)$ \\
\hline $\begin{array}{l}\text { Outpatient } \\
\text { Costs }\end{array}$ & $\begin{array}{c}1,829,139 \\
(33.7)\end{array}$ & $\begin{array}{c}349,807 \\
(35.5)\end{array}$ & $\begin{array}{c}976,038 \\
(38.8)\end{array}$ & $\begin{array}{c}503,294 \\
(29.4)\end{array}$ \\
\hline $\begin{array}{l}\text { Prescription } \\
\text { Costs }\end{array}$ & $\begin{array}{c}2,746,471 \\
(50.7)\end{array}$ & $\begin{array}{c}547,975 \\
(55.7)\end{array}$ & $\begin{array}{c}1,295,146 \\
(47.5)\end{array}$ & $\begin{array}{c}903,350 \\
(52.6)\end{array}$ \\
\hline Total Costs & $\begin{array}{c}5,422,419 \\
(100.0)\end{array}$ & $\begin{array}{c}983,877 \\
(100.0)\end{array}$ & $\begin{array}{c}2,724,347 \\
(100.0)\end{array}$ & $\begin{array}{c}1,714,195 \\
(100.0)\end{array}$ \\
\hline
\end{tabular}


Mean number of events (hospitalizations, ED visits, outpatient visits, prescriptions) and mean cost per event are listed in Table 5. T-tests and one-way ANOVA were used to determine statistically significant differences between the asthmaonly, COPD-only, and the both groups. The mean number of hospitalizations per recipient was highest for the both group (although not statistically significant), however, the hospitalization rate (per 10,000 enrollees) was the highest for the COPD-only group. Mean cost per hospitalization as determined from Medicare DRG reimbursements, was the highest for the COPD-only group, followed by the both group. The average LOS for the entire sample was 3.9 days, and was similar across the three disease groups. Mean number of ED episodes and the mean number of outpatient visits were significantly higher for the asthma-only and the both group as compared to the COPD-only group, however, mean cost per ED episode and mean cost per outpatient visit was higher for the asthma-only and the both group as compared to the COPD-only group. The mean number of prescriptions per recipient was highest for the both group at an average cost of $\$ 56$ per prescription. 
Table 5: Average Utilization/Costs and Utilization Rates

\begin{tabular}{|c|c|c|c|c|}
\hline Utilization Type & Overall Sample & Asthma-only & COPD-only & Both \\
\hline $\begin{array}{l}\text { Mean \# } \\
\text { Hospitalizations/ } \\
\text { Recipient }\end{array}$ & $1.3 \pm 0.1$ & $1.1 \pm 0.3$ & $1.3 \pm 0.7$ & $1.4 \pm 1.1$ \\
\hline $\begin{array}{l}\text { Hospitalization } \\
\text { Rate (Per } 10,000)\end{array}$ & 15.2 & 2.2 & 7.5 & 5.5 \\
\hline $\begin{array}{l}\text { Average LOS* } \\
\text { (days) }\end{array}$ & $3.9 \pm 1.9$ & $3.9 \pm 2.3$ & $3.9 \pm 1.9$ & $4.1 \pm 2.0$ \\
\hline $\begin{array}{l}\text { Mean Cost per } \\
\text { Hospitalization / } \\
\text { Recipient*\$ }^{(\$)}\end{array}$ & $3,091 \pm 1,069$ & $2,559 \pm 706$ & $3,316 \pm 1,295$ & $2,993 \pm 710$ \\
\hline $\begin{array}{l}\text { Mean \# ED } \\
\text { Episodes / } \\
\text { Recipient }\end{array}$ & $1.4 \pm 1.4$ & $1.3 \pm 0.8$ & $1.3 \pm 1.1$ & $1.8 \pm 2.1$ \\
\hline $\begin{array}{l}\text { ED Utilization } \\
\text { Rate (Per } 10,000)\end{array}$ & 75.3 & 8.1 & 49.2 & 17.9 \\
\hline $\begin{array}{l}\text { Mean Cost per } \\
\text { ED Episode / } \\
\text { Recipient*\$ }(\$)\end{array}$ & $87 \pm 130$ & $102 \pm 132$ & $79 \pm 127$ & $101 \pm 136$ \\
\hline $\begin{array}{l}\text { Mean \# } \\
\text { Outpatient } \\
\text { Visits/Recipient }\end{array}$ & $2.9 \pm 2.1$ & $3.0 \pm 3.6$ & $2.3 \pm 4.5$ & $7.3 \pm 8.2$ \\
\hline $\begin{array}{l}\text { Outpatient } \\
\text { utilization Rate } \\
\text { (Per } 1,000)\end{array}$ & 80.0 & 12.5 & 59.7 & 7.9 \\
\hline $\begin{array}{l}\text { Mean Cost per } \\
\text { Outpatient } \\
\text { Episode / } \\
\text { Recipient*\$ (\$) }\end{array}$ & $59.4 \pm 72.7$ & $76.5 \pm 105.3$ & $54.0 \pm 64.5$ & $73.1 \pm 61.5$ \\
\hline $\begin{array}{l}\text { Mean \# } \\
\text { Prescriptions/ } \\
\text { Recipient } \$ \\
\end{array}$ & $9.3 \pm 14.2$ & $10.8 \pm 12.1$ & $7.1 \pm 12.6$ & $18.7 \pm 19.8$ \\
\hline $\begin{array}{l}\text { Mean Cost per } \\
\text { Prescription / } \\
\text { Recipient*\$ (\$) }\end{array}$ & $43.9 \pm 61.5$ & $39.8 \pm 52.5$ & $39.6 \pm 59.7$ & $56.2 \pm 69.9$ \\
\hline
\end{tabular}

*Denominator included only enrollees who experienced the event

${ }^{\S}$ Significantly different $(\mathrm{p}<=0.05)$ 


\section{Results for Research Question 1}

The overall objective of research question 1 was to examine whether medication use was according to NHLBI guidelines for asthma and GOLD guidelines for COPD.

For Asthma-Only Group

According to the NHLBI guidelines, enrollees with asthma were classified into 4 groups:

Group I: Short-acting beta-agonist only;

Group II: Combination therapy without inhaled anti-inflammatory medications;

Group III: At least one prescription claim for inhaled anti-inflammatory therapy (ICS, inhaled cromolyn, or inhaled nedocromil)

Group IV: No prescription claims for chronic pulmonary disease-related medications

Figure 2 describes the pharmacotherapy use among the asthma-only group.

Tables 6 and 7 describe pharmacotherapy use among asthma-only enrollees by age group and gender.

As shown in Figure 2, only $6.2 \%$ of the recipients received short-acting betaagonist only. Almost one-third (29.5\%) of asthma-only enrollees received combination therapy (such as long-acting beta-agonists) without inhaled anti-inflammatory medications. More than half $(52.5 \%)$ received at least one prescription of an inhaled antiinflammatory medication, and only $12 \%$ of the respondents received no prescriptions for asthma-related medications.

Among patients with asthma receiving short-acting beta-agonists only, around $10 \%$ were in the 18 - 30 year age group, and more than half (52\%) were between 46 and 
64 years (Table 6). Among patients with asthma receiving ICS therapy (Group III) and combination therapy (Group II), almost $3 / 4^{\text {th }}$ were between $46-64$ years of age.

Females were responsible for almost 3/4ths of the pharmacotherapy use in Groups I - III (Table 7). Among patients with asthma not receiving any pharmacotherapy too, females constituted the majority. 
Figure 2. Pharmacotherapy Use Among Asthma-Only Enrollees

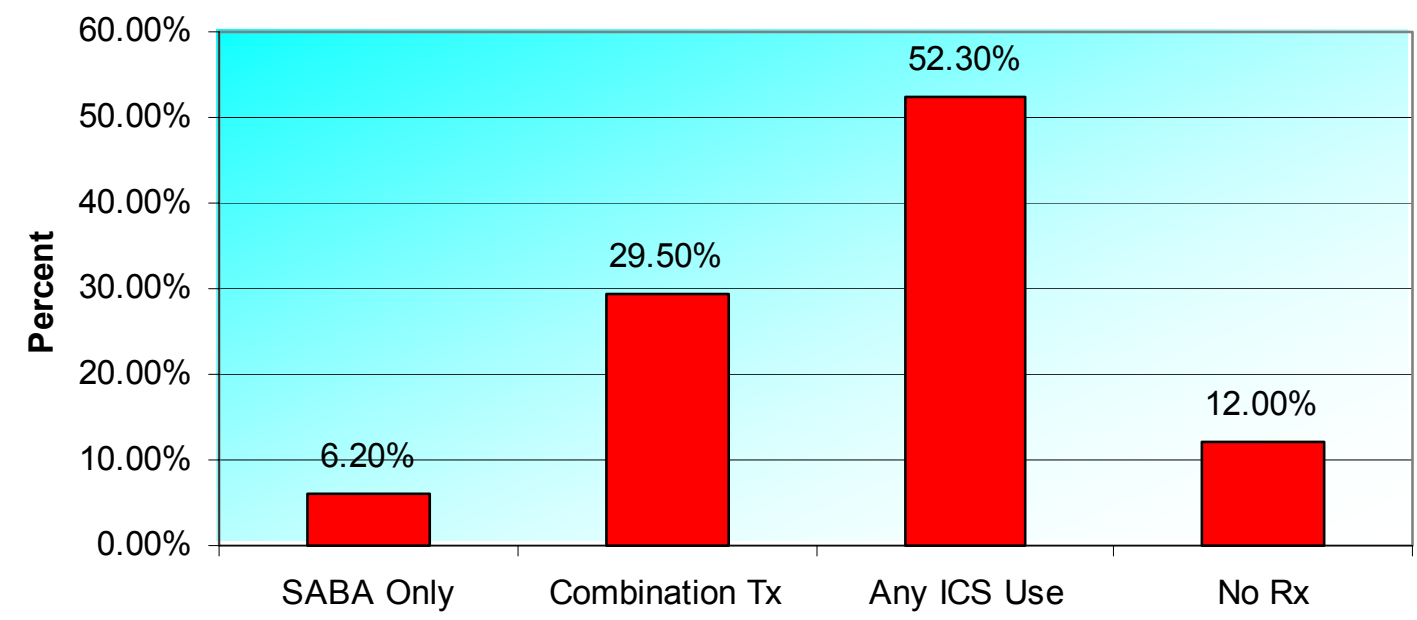


Table 6: Drug class by Age Group for Asthma-only Enrollees

\begin{tabular}{lcccc}
\hline $\begin{array}{l}\text { Age Range } \\
\text { (years) }\end{array}$ & Group I & Group II & Group III & Group IV \\
\hline $18-30$ & N (\%) & N (\%) & N (\%) & N (\%) \\
$31-45$ & $35(38.0)$ & $101(23.0)$ & $208(26.6)$ & $33(18.3)$ \\
$46-64$ & $48(52.2)$ & $321(72.9)$ & $538(68.9)$ & $140(77.8)$ \\
Total & $92(100.0)$ & $440(100.0)$ & $781(100.0)$ & $180(100.0)$ \\
\hline
\end{tabular}

Group I: Short-acting beta-agonist only; Group II: Combination therapy without inhaled anti-inflammatory medications; Group III: At least one prescription claim for inhaled anti-inflammatory therapy (ICS, inhaled cromolyn, or inhaled nedocromil); Group IV: No prescription claims for chronic pulmonary disease-related medications 
Table 7: Drug Class by Gender for Asthma-only Enrollees

\begin{tabular}{lcccc}
\hline Gender & Group I & Group II & Group III & Group IV \\
& N (\%) & N (\%) & N (\%) & N (\%) \\
\hline Male & $24(26.1)$ & $120(27.2)$ & $220(28.2)$ & $50(27.8)$ \\
Female & $68(73.9)$ & $320(72.8)$ & $561(71.8)$ & $130(72.2)$ \\
Total & $92(100.0)$ & $440(100.0)$ & $781(100.0)$ & $180(100.0)$ \\
\hline Group I: Short-acting beta-agonist only; Group II: Combination therapy without inhaled \\
anti-inflammatory medications; Group III: At least one prescription claim for inhaled \\
anti-inflammatory therapy (ICS, inhaled cromolyn, or inhaled nedocromil); Group IV: No \\
prescription claims for chronic pulmonary disease-related medications
\end{tabular}


For COPD-only group

The objective of research question 1 was to examine whether medication use was in accordance with GOLD guidelines. Figure 3 describes the pharmacotherapy use among the COPD-only group. COPD patients are classified as receiving bronchodilatorsonly, corticosteroids only, both bronchodilators and corticosteroids, and no COPD-related medications. Tables 8 and 9 describe pharmacotherapy use among COPD-only enrollees by age group and gender. As shown in Figure 3, 13.2\% of COPD-only patients received any bronchodilator. Of these, $7.5 \%$ received short-acting beta-agonist only. More than one-fifth $(20.5 \%)$ of the patients received any corticosteroid, of which $15.7 \%$ received at least one inhaled corticosteroid prescription. Almost one-third of all COPD-only patients received both bronchodilators as well as corticosteroids. More than one-third (36\%) of the COPD-only enrollees received no COPD-related medications. Females and COPDonly patients between $46-64$ years of age were responsible for the majority of prescription drug use. 
Figure 3. Pharmacotherapy Use Among COPD-Only Enrollees

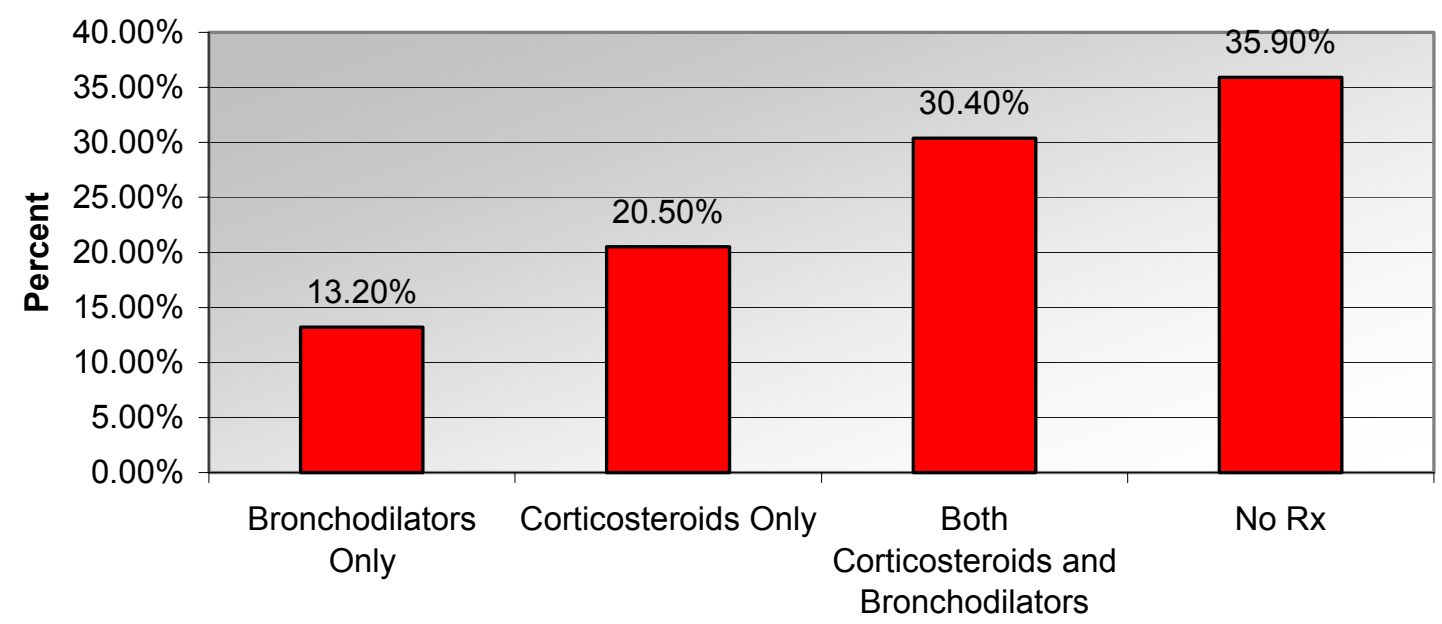


Table 8. Drug Class by Age Group for COPD-only Enrollees

\begin{tabular}{lcccc}
\hline $\begin{array}{l}\text { Age Range } \\
\text { (years) }\end{array}$ & $\begin{array}{c}\text { Bronchodilators } \\
\text { Only } \\
\text { N (\%) }\end{array}$ & $\begin{array}{c}\text { Corticosteroids } \\
\text { Only } \\
\mathbf{N}(\%)\end{array}$ & $\begin{array}{c}\text { Both } \\
\text { Bronchodilators } \\
\text { and } \\
\text { Corticosteroids } \\
\mathbf{N}(\%)\end{array}$ & $\begin{array}{c}\text { No COPD- } \\
\text { Related } \\
\text { Medication } \\
\text { N (\%) }\end{array}$ \\
\hline $18-30$ & $48(5.1)$ & $54(3.7)$ & $71(3.3)$ & $149(5.8)$ \\
$31-45$ & $263(27.7)$ & $339(23.1)$ & $611(28.1)$ & $649(25.3)$ \\
$46-64$ & $639(67.3)$ & $1072(73.2)$ & $1494(68.7)$ & $1,771(68.9)$ \\
Total & $950(100.0)$ & $1,465(100.0)$ & $2,176(100.0)$ & $2,570(100.0)$ \\
\hline
\end{tabular}


Table 9. Drug Class by Gender for COPD-only Enrollees

\begin{tabular}{lcccc}
\hline Gender & $\begin{array}{c}\text { Bronchodilators } \\
\text { Only } \\
\text { N (\%) }\end{array}$ & $\begin{array}{c}\text { Corticosteroids } \\
\text { Only } \\
\text { N (\%) }\end{array}$ & $\begin{array}{c}\text { Both } \\
\text { Bronchodilators } \\
\text { and } \\
\text { Corticosteroids } \\
\mathbf{N}(\%)\end{array}$ & $\begin{array}{c}\text { No COPD- } \\
\text { Related } \\
\text { Medication } \\
\text { N (\%) }\end{array}$ \\
\hline Male & $378(39.8)$ & $536(36.6)$ & $885(40.7)$ & $1,650(64.2)$ \\
Female & $572(60.2)$ & $929(63.4)$ & $1,291(59.3)$ & $920(35.8)$ \\
Total & $950(100.0)$ & $1,465(100.0)$ & $2,176(100.0)$ & $2,570(100.0)$ \\
\hline
\end{tabular}




\section{Both Asthma and COPD}

Since the both group consisted of enrollees having diagnosis codes for both asthma as well as COPD, pharmacotherapy use for this group was analyzed based on the NHLBI guidelines, since the GOLD guidelines do not have explicit recommendations for medication use unlike asthma. Figure 4 describes the pharmacotherapy use among the both group enrollees. Tables 10 and 11 describe pharmacotherapy use among the both group enrollees by age group and gender.

As shown in Figure 4, only 3.7\% of the recipients received short-acting betaagonist only. Almost one-third (30.3\%) of both-group enrollees received combination therapy without inhaled anti-inflammatory medications. More than half (59\%) received at least one prescription of an inhaled anti-inflammatory medication, and only $7.2 \%$ of the respondents received no prescriptions for asthma-related medications.

The age and gender distribution for pharmacotherapy use among the both group patients was similar to the asthma-only group (Tables 10 and 11). A majority of patients who received either ICS therapy (Group III) or other combination therapy were between 46 and 64 years of age. In groups I - III, females were responsible for the majority of pharmacotherapy use. Among patients not receiving any pharmacotherapy, females also constituted the majority. 
Figure 4. Pharmacotherapy Use Among Both Group Enrollees

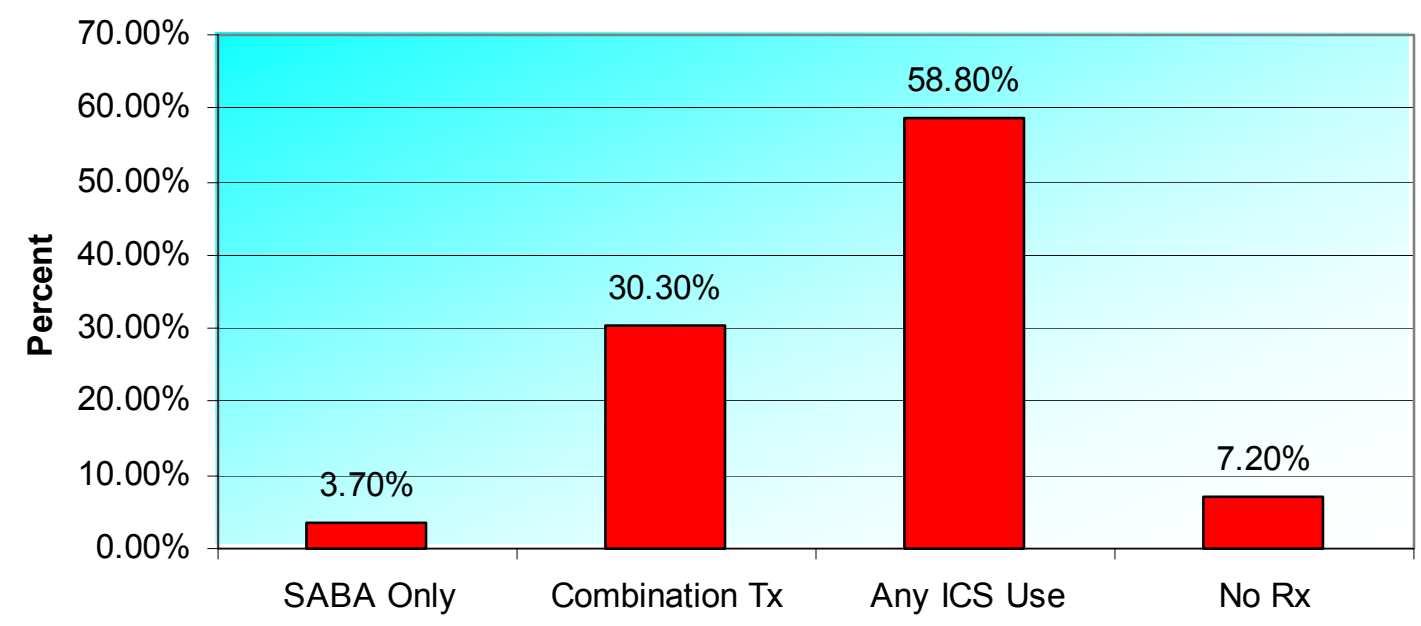


Table 10. Drug Class by Age Group for Both-group Enrollees

\begin{tabular}{|c|c|c|c|c|}
\hline $\begin{array}{l}\text { Age Range } \\
\text { (years) }\end{array}$ & $\begin{array}{l}\text { Group I } \\
\text { N (\%) }\end{array}$ & $\begin{array}{l}\text { Group II } \\
\text { N (\%) }\end{array}$ & $\begin{array}{c}\text { Group III } \\
\text { N }(\%)\end{array}$ & $\begin{array}{c}\text { Group IV } \\
\text { N (\%) }\end{array}$ \\
\hline $18-30$ & $2(5.7)$ & $12(4.1)$ & $17(3.1)$ & $0(0.0)$ \\
\hline $31-45$ & $12(34.3)$ & $60(21.0)$ & $103(18.6)$ & $13(19.4)$ \\
\hline $46-64$ & $21(64.0)$ & $213(74.9)$ & $433(78.3)$ & $54(80.6)$ \\
\hline Total & $35(100.0)$ & $285(100.0)$ & $553(100.0)$ & $67(100.0)$ \\
\hline
\end{tabular}

Group I: Short-acting beta-agonist only; Group II: Combination therapy without inhaled anti-inflammatory medications; Group III: At least one prescription claim for inhaled anti-inflammatory therapy (ICS, inhaled cromolyn, or inhaled nedocromil); Group IV: No prescription claims for chronic pulmonary disease-related medications 
Table 11. Drug Class by Gender for Both-Group Enrollees

\begin{tabular}{lcccc}
\hline Gender & Group I & Group II & Group III & Group IV \\
& N (\%) & N (\%) & N (\%) & N (\%) \\
\hline Male & $11(31.4)$ & $86(30.0)$ & $150(27.1)$ & $14(20.9)$ \\
Female & $24(68.6)$ & $199(70.0)$ & $403(72.9)$ & $53(79.1)$ \\
Total & $35(100.0)$ & $285(100.0)$ & $553(100.0)$ & $67(100.0)$ \\
\hline Group I: Short-acting beta-agonist only; Group II: Combination therapy without inhaled \\
anti-inflammatory medications; Group III: At least one prescription claim for inhaled \\
anti-inflammatory therapy (ICS, inhaled cromolyn, or inhaled nedocromil); Group IV: No
\end{tabular}




\section{Discussion for Research Question 1}

More than half of adult patients with asthma in the asthma-only group received a prescription for ICS therapy. Only $6 \%$ of patients with asthma in the asthma-only group received a prescription for short-acting beta agonists only. Almost 59\% of both group enrollees received at least one prescription for asthma or COPD-related medication, and only $3.7 \%$ of the patients used short-acting beta-agonists only. These findings suggest that a significant proportion of patients in the asthma-only and the both groups were receiving medications according to NHLBI guidelines. It has been shown that $58 \%$ of patients with asthma in a managed care plan used ICS therapy concomitantly with bronchodilators (Nestor et al, 1998). Physician prescribing for asthma-related medications has been shown to vary by physician specialty. The rate of ICS prescribing has been shown to be the lowest among nonspecialists (22\%), and highest among specialists (39\%) (Stempel et al, 1997). Also, asthma patients are more likely to use inhaled corticosteroids after an emergency room visit (Stempel et al, 1997). Patients in the both group experienced a higher number of ED visits than those in the asthma-only or the COPD-only groups. Thus, high ICS use in this group could be attributed to increasing disease severity, which may have led to an adverse outcome such as an ED visit or a hospitalization.

It is difficult to assess whether guidelines were followed for COPD-only patients, since the guidelines do not explicitly recommend the prophylactic use of any specific agent. It can be assumed that patients in this group who received a prescription for both bronchodilators as well as corticosteroids were more severe, than those receiving drugs from either class only. In the COPD-only group, around $36 \%$ of the patients with a 
medical claim did not have any pharmacy claims. This seems to suggest that these patients had mild COPD, and did not require pharmacotherapy, or that these patients were misdiagnosed as having COPD, which could potentially explain the lack of pharmacotherapy use in this group. In order to address this issue, a subanalysis was carried out to examine the average number of outpatient visits among those who received a prescription versus those who did not. For the entire Phase I sample, patients who received at least one prescription for an asthma or COPD-related medication experienced a significantly higher number of outpatient visits than those who did not receive any prescription (4.6 versus 1.9). In the asthma-only group, patients who received at least one prescription experienced an average of $3.5 \pm 4.0(\mathrm{SD})$ outpatient visits, as compared to $2.4 \pm 2.9$ (SD) visits for those who did not receive any prescription. For the COPDonly group, the differences were slightly higher, with patients receiving at least one prescription experiencing an average of $3.7 \pm 7.4(\mathrm{SD})$ outpatient visits, as compared to $1.7 \pm 2.3(\mathrm{SD})$ for those who did not receive any prescription. In the both group, patients with at least one prescription experienced an average of $8.3 \pm 9.0$ (SD) outpatient visits, however, patients without a prescription also experienced an average of $4.2 \pm 3.3$ (SD) outpatient visits. Based on this subanalysis, the probability of being potentially misclassified seems to be the highest for patients in the COPD-only group.

\section{Results for Research Question 2}

The objective of research question 2 was to examine the overall prevalence of asthma and COPD, and to examine prevalence by age group and gender. On average, there were 119,725 PEIA members between the ages of 18 - 64 years from 2001 through 
2003 in WV. Of these, gender data was available for 52,005 (43.4\%) males, and 63,817 (53.3\%) females. Also, based on age group, on average, 20,843 members were between $18-64$ years of age, 31,906 were between $31-45$ years of age, and 62,780 were between $45-64$ years of age. The following formulae were used to calculate prevalence.

Overall Prevalence of Asthma $=($ Number of asthma patients $/ 119,725) * 10,000$

$$
\begin{aligned}
& =(1,493 / 119,725) * 10,000 \\
& =124.7 / 10,000
\end{aligned}
$$

Overall Prevalence of COPD $=($ Number of COPD patients/119,725 $) * 10,000$

$$
\begin{aligned}
& =(7,161 / 119,725) * 10,000 \\
& =598 \cdot 1 / 10,000
\end{aligned}
$$

Overall Prevalence of Patients having Both Asthma and COPD $=($ Number of Patients with Both Asthma and COPD/119,725)*10,000

$$
\begin{aligned}
& =(940 / 119,725) * 10,000 \\
& =78.51 / 10,000
\end{aligned}
$$

Similarly, the prevalence for the asthma-only, COPD-only, and both group patients was measured for each age group and gender. The denominators used for these analyses were the number of PEIA covered members in each age group, or in each gender category. The results of this analysis are presented in Table 12 . The prevalence of COPD was significantly higher than asthma for each age group as well as gender. The prevalence of asthma as well as COPD increased with age, and was highest in the $46-64$ year age group. The prevalence of asthma and COPD was significantly higher in females than males. 
Table 12: Prevalence of Asthma and COPD in WV PEIA Members

\begin{tabular}{lccc}
\hline Characteristic & \multicolumn{2}{c}{ Prevalence Per 10,000 WV PEIA Members } \\
\cline { 2 - 4 } & Asthma-Only & COPD-Only & Both Asthma and \\
& & & COPD \\
Age Group & 41.3 & 154.5 & 13.0 \\
$18-30$ years & 130.7 & 583.6 & 61.4 \\
$31-45$ years & 157.7 & 792.8 & 114.2 \\
$46-64$ years & & & 410.7 \\
Gender & 81.1 & 522.8 & \\
Male & 167.8 & 854.1 & \\
Female & & & \\
\hline
\end{tabular}




\section{Discussion for Research Question 2}

Based on the analyses, the null hypothesis A that there is no difference in prevalence by age group and gender is rejected for the asthma-only, COPD-only as well as the both groups.

The prevalence rate for asthma in 2001 was 730 per 10,000 persons (CDC, 2001). Thus, the prevalence of asthma in WV PEIA employees was significantly lower than national estimates. Combining the asthma-only and the both group yielded a total asthma prevalence of 203 per 10,000 persons, which is slightly higher than the asthma prevalence in WV Medicaid for enrollees in the 18 - 64 age group (177 / 10,000 persons), according to a recent study (Joshi and Smith, 2003). Asthma prevalence was higher in females compared to males, which was consistent with national estimates (Stempel et al, 2001 and CDC, 2001). The overall prevalence of COPD in the WV PEIA sample was similar to national estimates. Findings from the National Health Interview Survey (NHIS) suggested that the prevalence of chronic bronchitis and emphysema in the United States was 619 per 10,000 persons in 1993 (NHIS, 1993). Data from the Lung Health Study conducted by the NHLBI in 1993 showed that the prevalence of emphysema is higher in males $(97 / 10,000$ in males vs. $6 / 10,000$ in females), whereas the prevalence of chronic bronchitis is higher in females $(630 / 10,000$ in females vs. 445/10,000 in males) (Hurd, 2000). In WV PEIA members, the prevalence of COPD was significantly higher in females as compared to males. 


\section{Results for Research Question 3}

This study objective measures the extent of nonadherence to maintenance medications using indices such as the MPR and median gap. The mean MPR for the entire sample was $0.17 \pm 0.3$ and the median gap was $561 \pm 435$ days. The MPR and median gap for each group are shown in Table 13. Both MPR and median gap values indicate that the COPD-group was the least adherent, and the both group was the most adherent. The use of both indices enabled the validation of trend in nonadherence among the three groups. Spearman's rank correlation coefficient between MPR and median gap was statistically significant for the overall sample as well as each disease subgroup.

The mean TMS for the overall sample was $18.1 \pm 16.7$, and the mean CMS for the overall sample was $4.6 \pm 5.3$. Table 13 shows the TMS and CMS values for the 3 groups. Differences in TMS and CMS scores between the asthma-only and the COPD only groups were not significant, however, the both group had significantly higher TMS and CMS scores than the asthma-only and the COPD-only groups. 
Table 13: Distribution of Medication Adherence-Related Variables

\begin{tabular}{lcccc}
\hline Variable & Overall Sample & Asthma-only & COPD-only & Both Group \\
\hline Mean MPR $^{\mathrm{a}}$ & 0.17 & 0.35 & 0.10 & 0.40 \\
Median Gap & & & & \\
$\mathrm{TMS}^{\mathrm{c}}$ & 561 & 306 & 653 & 268 \\
$\mathrm{CMS}^{\mathrm{d}}$ & 18.1 & 17.8 & 17.6 & 22.3 \\
& 4.6 & 4.7 & 4.4 & 6.3 \\
\hline
\end{tabular}

${ }^{\mathrm{a} O n e-w a y ~ A N O V A ~ s h o w e d ~ s t a t i s t i c a l l y ~ s i g n i f i c a n t ~ d i f f e r e n c e s ~ i n ~ M e a n ~ M P R ~ a m o n g ~ t h e ~}$

3 groups

${ }^{\mathrm{b}}$ One-way ANOVA showed statistically significant differences in Median Gap between the Asthma-only group and the COPD-only Group, and the Asthma-only group and the Both group

${ }^{\mathrm{c}, \mathrm{d}}$ One-way ANOVA showed significantly higher values for the Both Group as compared with the Asthma-only and the COPD-only groups 


\section{Discussion for Research Question 3}

Based on the analyses, the adherence was highest for the both group, and lowest for the COPD-only group. These results are not surprising since NHLBI guidelines recommend the use of ICS pharmacotherapy as the primary prophylactic medication for asthma, as well as the use of other maintenance drugs as add-on prophylactic medications. Due to a lack of specific recommendations for patients with COPD, the MPR was the lowest for the COPD-only group. The MPR was the highest in patients having both asthma and COPD, indicating an increased use of maintenance therapy, which was a function of increasing disease severity.

In another study, patients with asthma have been classified as being poor adherent (up to 2), moderate adherent (3-6), or good adherent (at least 7), based on the number of prescriptions for maintenance medications per year (Luskin et al, 2001). Based on this criteria, for the overall Phase I sample, only $7.8 \%$ were classified as adherent, $8.8 \%$ were classified as moderate adherent, and the majority (83\%) were classified as poor adherent. Among the three disease groups, the proportion of patients classified as poor adherent was the highest for the COPD-only group (almost 90\%), followed by the asthma-only group $(72 \%)$. The proportion of patients classified as poor adherent was lowest for the both group (51\%). Overall, the prevalence of medication adherence in WV PEIA members in the asthma-only and COPD-only group was lower than national estimates, based on refill claims data. Generally, medication adherence rates for asthma range from 3 to $46 \%$, with the mean adherence being less than 50\% (Kelloway et al, 1994 and Fish and Lung, 2001). For the both group, the prevalence of medication adherence was similar to the national average. 


\section{Results for Research Question 4}

The objective of research question 4 was to examine the impact of medication adherence on healthcare utilization. The dependent variables were number of adverse outcomes (hospitalizations and/or ED visits), number of outpatient visits, and LOS. Since the number of adverse outcomes, and the number of outpatient visits represent count data, either Poisson or negative binomial regression was used to measure the impact of medication adherence - the primary predictor, on utilization. OLS regression was used to measure the impact of medication nonadherence on LOS. Of the total Phase I sample, only 182 patients (from the 3 disease groups combined) experienced at least one hospitalization. Since OLS regression requires at least $15-20$ subjects per predictor variable, there were not enough cases to run separate models for each disease group. Hence, a combined model with all 3 disease groups was run with LOS as the dependent variable.

\section{Number of Adverse Outcomes}

The results of the Poisson regression models measuring the impact of medication adherence on the number of adverse outcomes are presented in Tables $14-21$. For the asthma-only group, when MPR was used as the primary predictor, TMS and asthma severity were significant predictors of the number of adverse outcomes (Table 14). Every unit increase in TMS was associated with a $1.6 \%$ increase in the number of adverse outcomes. Patients with level I asthma severity experienced a 116\% lower number of adverse outcomes than patients with level V asthma severity, and patients with level III asthma severity experienced a $133 \%$ lower number of adverse outcomes than level V asthma patients. When MPR was replaced by median gap (Table 15), the model did not 
change significantly. Median gap was not a significant predictor of the number of adverse outcomes. 
Table 14: Poisson Regression Model with Predictors of Number of Adverse

Outcomes using MPR Adherence for the Asthma-Only Group

\begin{tabular}{|c|c|c|c|c|c|}
\hline Predictor & $\mathrm{e}^{\mathrm{x}}$ & Estimate & SE & $\begin{array}{c}\text { Chi-Square } \\
\text { Statistic }\end{array}$ & Significance \\
\hline Age & -- & -0.0116 & 0.0086 & 1.82 & 0.1779 \\
\hline Female Sex & -- & 0.1803 & 0.1893 & 0.91 & 0.3410 \\
\hline TMS & 1.0163 & 0.0162 & 0.0071 & 5.20 & $0.0227 *$ \\
\hline CMS & -- & -0.0353 & 0.0268 & 1.73 & 0.1880 \\
\hline \multicolumn{6}{|c|}{ Asthma Severity } \\
\hline Level I & 2.1586 & -0.7695 & 0.3628 & 4.50 & $0.0339 *$ \\
\hline Level II & -- & -0.1265 & 0.7850 & 0.03 & 0.8720 \\
\hline Level III & 2.3331 & -0.8472 & 0.3876 & 4.78 & $0.0289 *$ \\
\hline Level IV & -- & -0.1791 & 0.3277 & 0.30 & 0.5846 \\
\hline Level V & -- & -- & -- & -- & -- \\
\hline MPR & -- & -0.0408 & 0.2413 & 0.03 & 0.8657 \\
\hline
\end{tabular}

*Significant at $\mathrm{p}<=0.05$; SE: Standard Error; $\mathrm{e}^{\mathrm{x}}$ : Exponential of the estimate; Log

Likelihood $=-464.92 ;$ Deviance $=0.58, \mathrm{~N}=1,413 ;$ Chi-square $=806.9 ;$ Sig. $=0.000$ 
Table 15: Poisson Regression Model with Predictors of Number of Adverse

Outcomes using Median Gap Adherence for the Asthma-Only Group

\begin{tabular}{lccccc}
\hline Predictor & $\mathbf{e}^{\mathbf{x}}$ & Estimate & SE & Chi-Square & Significance \\
& & & & Statistic & \\
\hline Age & -- & -0.0115 & 0.0086 & 1.81 & 0.1784 \\
Female Sex & -- & 0.1837 & 0.1894 & 0.94 & 0.3320 \\
TMS & 1.0165 & 0.0164 & 0.0071 & 5.43 & $0.0198^{*}$ \\
CMS & -- & -0.0356 & 0.0266 & 1.80 & 0.1803 \\
Asthma Severity & & & & & $0.0301^{*}$ \\
$\quad$ Level I & 2.1643 & -0.7721 & 0.3560 & 4.70 & 0.8628 \\
$\quad$ Level II & -- & -0.1347 & 0.7792 & 0.03 & $0.0342^{*}$ \\
$\quad$ Level III & 2.2880 & -0.8277 & 0.3909 & 4.48 & 0.5989 \\
$\quad$ Level IV & -- & -0.1710 & 0.3251 & 0.28 & 0.7966 \\
$\quad$ Level V & -- & & & & 0.01 \\
Median Gap & -- & 0.0200 & 0.2744 &
\end{tabular}

*Significant at $\mathrm{p}<=0.05$; SE: Standard Error; $\mathrm{e}^{\mathrm{x}}$ : Exponential of the estimate; Log

Likelihood $=-464.86 ;$ Deviance $=0.58, \mathrm{~N}=1,413 ;$ Chi-square $=2786.4 ;$ Sig. $=0.000$ 
For the COPD-only group, being female was associated with a 33\% reduction in the number of adverse outcomes as compared to males. A $100 \%$ increase in adherence as measured by the MPR was associated with $360 \%$ increase in the number of adverse outcomes (Table 16). When MPR was replaced by median gap (Table 17), the model did not change significantly. An increase in median gap by 1 day (reduced medication adherence) was associated with a $0.6 \%$ decrease in the number of adverse outcomes. 
Table 16: Poisson Regression Model with Predictors of Number of Adverse Outcomes using MPR Adherence for the COPD-Only Group

\begin{tabular}{lccccc}
\hline Predictor & $\mathbf{e}^{\mathbf{x}}$ & Estimate & SE & Chi-Square & Significance \\
& & & & Statistic \\
& & & & \\
& & -0.0022 & 0.0038 & 0.33 & 0.5639 \\
Age & 1.3328 & -0.2873 & 0.0688 & 17.44 & $0.0001^{*}$ \\
TMS & -- & 0.0031 & 0.0030 & 1.08 & 0.2993 \\
CMS & -- & 0.0124 & 0.0096 & 1.64 & 0.2001 \\
MPR & 4.60 & 1.5268 & 0.1011 & 227.88 & $0.0001^{*}$ \\
\hline
\end{tabular}

$*$ Significant at $\mathrm{p}<=0.05 ; \mathrm{SE}$ : Standard Error; $\mathrm{e}^{\mathrm{x}}$ : Exponential of the estimate; Log
Likelihood $=-2524.0$; Deviance $=0.60, \mathrm{~N}=7,161$; Chi-square $=12392.67$; Sig. $=0.000$ 
Table 17: Poisson Regression Model with Predictors of Number of Adverse Outcomes using Median Gap Adherence for the COPD-Only Group

\begin{tabular}{lccccc}
\hline Predictor & $\mathbf{e}^{\mathbf{x}}$ & Estimate & SE & Chi-Square & Significance \\
& & & \multicolumn{3}{c}{ Statistic } \\
\hline Age & -- & 0.0017 & 0.0038 & 0.21 & 0.6459 \\
Female Sex & 1.3627 & -0.3095 & 0.0687 & 20.27 & $0.0001^{*}$ \\
TMS & 1.0007 & 0.0007 & 0.0030 & 0.05 & 0.8275 \\
CMS & -- & 0.0287 & 0.0092 & 9.76 & 0.1008 \\
Median Gap & 1.0006 & -0.0006 & 0.0001 & 59.34 & $0.0001^{*}$ \\
\hline
\end{tabular}

*Significant at $\mathrm{p}<=0.05 ; \mathrm{SE}$ : Standard Error; $\mathrm{e}^{\mathrm{x}}$ : Exponential of the estimate; Log

Likelihood $=-2591.0 ;$ Deviance $=0.62, \mathrm{~N}=7,161 ;$ Chi-square $=15168.32 ;$ Sig. $=0.000$ 
For the both group, TMS and female sex were significant predictors of the number of adverse outcomes. Every unit increase in TMS was associated with a 5.1\% increase in the number of adverse outcomes. Being female was associated with a $26 \%$ lower number of adverse outcomes (Table 18). The model did not change when MPR was replaced by median gap (Table 19). 
Table 18: Poisson Regression Model with Predictors of Number of Adverse Outcomes using MPR Adherence for the Both Group

\begin{tabular}{lccccc}
\hline Predictor & $\mathbf{e}^{\mathbf{x}}$ & Estimate & SE & Chi-Square & Significance \\
& & & & Statistic \\
& & & & & \\
\hline Age & -- & 0.0016 & 0.0054 & 0.08 & 0.7729 \\
Female Sex & 1.2569 & -0.2287 & 0.1001 & 5.22 & $0.0224^{*}$ \\
TMS & 1.0510 & 0.0149 & 0.0000 & 15.01 & $0.0001^{*}$ \\
CMS & -- & -0.0091 & 0.0038 & 0.57 & 0.4517 \\
MPR & -- & 0.0761 & 0.0121 & 0.37 & 0.5418 \\
& & & & & \\
\hline
\end{tabular}

\footnotetext{
*Significant at $\mathrm{p}<=0.05 ;$ SE: Standard Error; $\mathrm{e}^{\mathrm{x}}$ : Exponential of the estimate; Log

Likelihood $=-771.37$; Deviance $=1.70, \mathrm{~N}=940 ;$ Chi-square $=4217.81 ;$ Sig. $=0.000$
} 
Table 19: Poisson Regression Model with Predictors of Number of Adverse Outcomes using Median Gap Adherence for the Both Group

\begin{tabular}{lccccc}
\hline Predictor & $\mathbf{e}^{\mathbf{x}}$ & Estimate & SE & Chi-Square & Significance \\
& & & & Statistic \\
& & & & & \\
\hline Age & -- & 0.0018 & 0.0053 & 0.11 & 0.7391 \\
Female Sex & 1.2612 & -0.2321 & 0.0998 & 5.41 & $0.0200^{*}$ \\
TMS & 1.0147 & 0.0146 & 0.0039 & 14.30 & $0.0002^{*}$ \\
CMS & -- & -0.0082 & 0.0120 & 0.47 & 0.4940 \\
Median Gap & -- & -0.0001 & 0.0001 & 0.72 & 0.3966 \\
\hline
\end{tabular}

*Significant at $\mathrm{p}<=0.05$; SE: Standard Error; $\mathrm{e}^{\mathrm{x}}$ : Exponential of the estimate; Log

Likelihood $=-771.19 ;$ Deviance $=1.79, \mathrm{~N}=940 ;$ Chi-square $=4175.40 ;$ Sig. $=0.000$ 
In order to adjust for confounding by disease severity, combined group models was run with dummy codes for the COPD-only and both groups, with asthma-only as the reference (Tables 20 and 21). In this model, female sex, TMS, COPD dummy, asthma dummy, and MPR were significant predictors of the number of adverse outcomes. Being female was associated with a $26 \%$ lower number of adverse outcomes. As compared to COPD-only patients, asthma patients experienced a 40\% lower number of adverse outcomes. As compared to patients having both asthma and COPD, patients having asthma-only experienced a $339 \%$ lower number of adverse outcomes. A $100 \%$ increase in medication adherence (as MPR increased from 0 to 1 ) was associated with a $117 \%$ increase in the number of adverse outcomes. When MPR was replaced by median gap as primary predictor, the model did not change significantly (Table 21). An increase in median gap by 1 day was associated with a slightly lower number of adverse outcomes. 
Table 20: Poisson Regression Model with Predictors of Number of Adverse

Outcomes using MPR Adherence for the Entire Phase I Sample

\begin{tabular}{lccccc}
\hline Predictor & $\mathbf{e}^{\mathbf{x}}$ & Estimate & SE & Chi-Square & Significance \\
& & & & Statistic \\
& & & & \\
\hline Age & -- & -0.0017 & 0.0029 & 0.33 & 0.567 \\
Female Sex & 1.2664 & -0.2362 & 0.0537 & 19.33 & $0.000^{*}$ \\
TMS & 1.0092 & 0.0092 & 0.0022 & 17.85 & $0.000^{*}$ \\
CMS & -- & 0.0023 & 0.0072 & 0.10 & 0.748 \\
COPD Dummy & 1.4074 & -0.3418 & 0.0912 & 14.03 & $0.002 *$ \\
Both Dummy & 4.3973 & -1.4810 & 0.0934 & 251.20 & $0.000^{*}$ \\
MPR & 2.1749 & 0.7770 & 0.0787 & 97.46 & $0.000^{*}$ \\
\hline
\end{tabular}

\footnotetext{
*Significant at $\mathrm{p}<=0.05 ;$ SE: Standard Error; $\mathrm{e}^{\mathrm{x}}$ : Exponential of the estimate; Log

Likelihood $=-3852.12 ;$ Deviance $=0.72, \mathrm{~N}=9,594 ;$ Chi-square $=21213.5 ;$ Sig. $=0.000$
} 
Table 21: Poisson Regression Model with Predictors of Number of Adverse

Outcomes using Median Gap Adherence for the Entire Phase I Sample

\begin{tabular}{lccccc}
\hline Predictor & $\mathbf{e}^{\mathbf{x}}$ & Estimate & SE & Chi-Square & Significance \\
& & & & Statistic & \\
\hline Age & -- & 0.0002 & 0.0029 & 0.01 & 0.9412 \\
Female Sex & 1.2885 & -0.2535 & 0.0537 & 22.27 & $0.0001^{*}$ \\
TMS & 1.0077 & 0.0077 & 0.0022 & 11.99 & $0.0005^{*}$ \\
CMS & -- & 0.0104 & 0.0070 & 2.22 & 0.1359 \\
COPD Dummy & 1.2977 & -0.2606 & 0.0906 & 8.27 & $0.0040^{*}$ \\
Both Dummy & 4.5145 & -1.5073 & 0.0933 & 260.95 & $0.0001^{*}$ \\
Median Gap & 1.0004 & -0.0004 & 0.0001 & 38.47 & $0.0001^{*}$ \\
\hline
\end{tabular}

*Significant at $\mathrm{p}<=0.05 ;$ SE: Standard Error; $\mathrm{e}^{\mathrm{x}}$ : Exponential of the estimate; Log

Likelihood $=-3878.93 ;$ Deviance $=0.73, \mathrm{~N}=9,594 ;$ Chi-square $=22775.8 ;$ Sig. $=0.000$ 


\section{Number of Outpatient Visits}

When Poisson regression was used to identify factors that impact the number of outpatient visits using medication adherence as the primary predictor, the deviance in the models was significantly greater than 1 , which indicates that there is overdispersion. This means that the true variance is greater than the mean. In order to account for overdispersion, negative binomial regression is recommended. Thus, for the following models predicting the number of outpatient visits, negative binomial regression was used. Note that the deviance in each of the following models is close to 1.

For the asthma only group, age, female sex, asthma severity, and medication adherence were significant predictors of the total number of outpatient visits. In the model using MPR as primary predictor (Table 22), every unit increase in age was associated with a $0.75 \%$ higher number of outpatient visits. Being female was associated with a 30\% higher number of outpatient visits. Every unit increase in TMS increased the number of outpatient visits by $0.6 \%$. The number of outpatient visits were $59 \%$ lower in level I asthma patients, and almost $41 \%$ lower in level III asthma patients, as compared with level V asthma patients. Increase in medication adherence based on MPR was associated with an $86 \%$ increase in the number of outpatient visits. 
Table 22: Negative Binomial Regression Model with Predictors of Number of Outpatient Visits using MPR Adherence for the Asthma-Only Group

\begin{tabular}{lccccc}
\hline Predictor & $\mathbf{e}^{\mathbf{x}}$ & Estimate & SE & Chi-Square & Significance \\
& & & & Statistic & \\
\hline Age & 1.0075 & 0.0075 & 0.0025 & 9.27 & $0.0023^{*}$ \\
Female Sex & 1.2978 & 0.2607 & 0.0526 & 24.60 & $0.0001^{*}$ \\
TMS & 1.0063 & 0.0063 & 0.0023 & 7.57 & $0.0059^{*}$ \\
CMS & -- & -0.0139 & 0.0071 & 3.89 & 0.0685 \\
Asthma Severity & & & & & \\
$\quad$ Level I & 1.5972 & -0.4683 & 0.1128 & 17.22 & $0.0000^{*}$ \\
$\quad$ Level II & -- & -0.2053 & 0.2581 & 0.63 & 0.4265 \\
$\quad$ Level III & 1.4083 & -0.3424 & 0.1141 & 9.01 & $0.0027^{*}$ \\
$\quad$ Level IV & -- & -0.1803 & 0.1073 & 2.82 & 0.0928 \\
$\quad$ Level V & -- & -- & & & \\
MPR & 1.8574 & 0.6192 & 0.0656 & 89.07 & $0.0001^{*}$ \\
\hline
\end{tabular}

\footnotetext{
*Significant at $\mathrm{p}<=0.05$; SE: Standard Error; $\mathrm{e}^{\mathrm{x}}$ : Exponential of the estimate; Log

Likelihood $=1086.35 ;$ Deviance $=0.87, \mathrm{~N}=1,413 ;$ Chi-square $=2188.90 ;$ Sig. $=0.000$
} 
When MPR was replaced by median gap, the results did not change significantly (Table 23). Every unit increase in median gap was associated with a $0.02 \%$ decrease in the number of outpatient visits. 
Table 23: Negative Binomial Regression Model with Predictors of Number of Outpatient Visits using Median Gap Adherence for the Asthma-Only Group

\begin{tabular}{lccccc}
\hline Predictor & $\mathbf{e}^{\mathbf{x}}$ & Estimate & SE & Chi-Square & Significance \\
& & & & Statistic & \\
\hline Age & 1.0083 & 0.0083 & 0.0025 & 10.99 & $0.0009^{*}$ \\
Female Sex & 1.2730 & 0.2416 & 0.0530 & 20.79 & $0.0001^{*}$ \\
TMS & -- & 0.0038 & 0.0023 & 2.79 & 0.0949 \\
CMS & -- & -0.0057 & 0.0071 & 0.64 & 0.4239 \\
Asthma Severity & & & & & \\
$\quad$ Level I & 1.8023 & -0.5891 & 0.1126 & 27.36 & $0.0001^{*}$ \\
$\quad$ Level II & -- & -0.3899 & 0.2607 & 2.24 & 0.1349 \\
$\quad$ Level III & 1.5212 & -0.4195 & 0.1159 & 13.11 & $0.0003^{*}$ \\
$\quad$ Level IV & 1.3211 & -0.2785 & 0.1081 & 6.64 & $0.0100^{*}$ \\
$\quad$ Level V & -- & -- & & & \\
Median Gap & 1.0002 & -0.0002 & 0.0400 & 3.23 & $0.0001^{*}$ \\
\hline
\end{tabular}

\footnotetext{
*Significant at $\mathrm{p}<=0.05$; SE: Standard Error; $\mathrm{e}^{\mathrm{x}}$ : Exponential of the estimate; $\log$

Likelihood $=1067.1637 ;$ Deviance $=0.88, \mathrm{~N}=1,413 ;$ Chi-square $=2151.51 ;$ Sig. $=$ 0.000
} 
For the COPD-only model, all the independent variables were significant predictors of the number of outpatient visits (Table 24). Every unit increase in age was associated with a $1.2 \%$ increase in the number of outpatient visits. Being female was associated with a $5.5 \%$ increase in the number of outpatient visits. Every unit increase in TMS was associated with a $0.36 \%$ decrease and every unit increase in CMS was associated with a $2.8 \%$ increase in the number of outpatient visits. Increase in medication adherence by $100 \%$ (since MPR measures adherence from 0 to 1 ) was associated with a $276 \%$ increase in the number of outpatient visits. When MPR was replaced by median gap, female sex was no longer a significant predictor of the number of outpatient visits (Table 25). Every unit increase in median gap was associated with a $0.06 \%$ decrease in the number of outpatient visits. Thus, the number of outpatient visits decreased with increasing adherence. 
Table 24: Negative Binomial Regression Model with Predictors of Number of Outpatient Visits using MPR Adherence for the COPD-Only Group

\begin{tabular}{lccccc}
\hline Predictor & $\mathbf{e}^{\mathbf{x}}$ & Estimate & $\mathbf{S E}$ & $\begin{array}{c}\text { Chi-Square } \\
\text { Statistic }\end{array}$ & Significance \\
& & & \multicolumn{5}{c}{} \\
\hline Age & 1.0122 & 0.0122 & 0.0012 & 96.40 & $0.0001^{*}$ \\
Female Sex & 1.0547 & 0.0533 & 0.0233 & 5.23 & $0.0222^{*}$ \\
TMS & 1.0036 & -0.0036 & 0.0012 & 9.23 & $0.0024^{*}$ \\
CMS & 1.0276 & 0.0273 & 0.0038 & 51.84 & $0.0001^{*}$ \\
MPR & 3.7678 & 1.3265 & 0.0446 & 885.71 & $0.0001^{*}$ \\
\hline
\end{tabular}

\footnotetext{
*Significant at $\mathrm{p}<=0.05$; SE: Standard Error; $\mathrm{e}^{\mathrm{x}}$ : Exponential of the estimate; Log Likelihood $=2897.26$; Deviance $=0.77, \mathrm{~N}=7,161$; Chi-square $=14920.47$; Sig. $=$ 0.000
} 
Table 25: Negative Binomial Regression Model with Predictors of Number of Outpatient Visits using Median Gap Adherence for the COPD-Only Group

\begin{tabular}{cccccc}
\hline Predictor & $\mathbf{e}^{\mathbf{x}}$ & Estimate & $\mathbf{S E}$ & $\begin{array}{c}\text { Chi-Square } \\
\text { Statistic }\end{array}$ & Significance \\
& & & & & \\
\hline Age & 1.014 & 0.0142 & 0.0013 & 124.59 & $0.0001^{*}$ \\
Female Sex & -- & 0.0321 & 0.0238 & 1.82 & 0.1778 \\
TMS & 1.0069 & -0.0069 & 0.0012 & 31.06 & $0.0001^{*}$ \\
CMS & 1.049 & 0.0485 & 0.0038 & 161.33 & $0.0001^{*}$ \\
Median Gap & 1.0006 & -0.0006 & 0.0000 & 413.17 & $0.0001^{*}$ \\
\hline
\end{tabular}

*Significant at $\mathrm{p}<=0.05$; SE: Standard Error; $\mathrm{e}^{\mathrm{x}}$ : Exponential of the estimate; Log Likelihood $=2644.94 ;$ Deviance $=0.79, \mathrm{~N}=7,161 ;$ Chi-square $=17876.02 ;$ Sig. $=$ 0.000 
For the both group, age, female sex, TMS, and medication adherence were significant predictors of the number of outpatient visits (Tables 26 and 27). In both models, the number of outpatient visits increased with age, and being female was associated with an almost $18 \%$ increase in the number of outpatient visits. Increase in medication adherence by $100 \%$, as indicated by the MPR, was associated with a $70 \%$ increase in the number of outpatient visits. With every unit increase in median gap, the number of outpatient visits decreased by $0.05 \%$ (Table 27 ). 
Table 26: Negative Binomial Regression Model with Predictors of Number of Outpatient Visits using MPR Adherence for the Both Group

\begin{tabular}{lccccc}
\hline Predictor & $\mathbf{e}^{\mathbf{x}}$ & Estimate & $\mathbf{S E}$ & $\begin{array}{c}\text { Chi-Square } \\
\text { Statistic }\end{array}$ & Significance \\
& & & \multicolumn{4}{c}{} \\
\hline Age & 1.0144 & 0.0143 & 0.0028 & 25.47 & $0.0001^{*}$ \\
Female Sex & 1.1792 & 0.1649 & 0.0575 & 8.24 & $0.0041^{*}$ \\
TMS & 1.0077 & 0.0077 & 0.0026 & 8.72 & $0.0031^{*}$ \\
CMS & -- & -0.0011 & 0.0072 & 0.02 & 0.8816 \\
MPR & 1.7023 & 0.5320 & 0.0679 & 61.32 & $0.0001^{*}$ \\
\hline
\end{tabular}

\footnotetext{
*Significant at $\mathrm{p}<=0.05$; SE: Standard Error; $\mathrm{e}^{\mathrm{x}}$ : Exponential of the estimate; Log

Likelihood $=8335.6 ;$ Deviance $=0.97, \mathrm{~N}=940 ;$ Chi-square $=1723.67 ;$ Sig. $=0.000$
} 
Table 27: Negative Binomial Regression Model with Predictors of Number of Outpatient Visits using Median Gap Adherence for the Both Group

\begin{tabular}{lccccc}
\hline Predictor & $\mathbf{e}^{\mathbf{x}}$ & Estimate & $\mathbf{S E}$ & $\begin{array}{c}\text { Chi-Square } \\
\text { Statistic }\end{array}$ & Significance \\
& & & \multicolumn{4}{c}{} \\
\hline Age & 1.016 & 0.0163 & 0.0028 & 32.87 & $0.0001^{*}$ \\
Female Sex & 1.165 & 0.1530 & 0.0578 & 7.00 & $0.0081^{*}$ \\
TMS & 1.005 & 0.0057 & 0.0026 & 4.63 & $0.0315^{*}$ \\
CMS & -- & 0.0082 & 0.0071 & 1.31 & 0.2515 \\
Median Gap & 1.0005 & -0.0005 & 0.0001 & 46.55 & $0.0001^{*}$ \\
\hline
\end{tabular}

*Significant at $\mathrm{p}<=0.05$; SE: Standard Error; $\mathrm{e}^{\mathrm{x}}$ : Exponential of the estimate; Log Likelihood $=8327.61 ;$ Deviance $=0.98, \mathrm{~N}=940 ;$ Chi-square $=1520.33 ;$ Sig. $=0.000$ 
In order to adjust for confounding by disease severity, the models were rerun with dummy codes for COPD-only and both asthma and COPD as compared to patients with asthma only. In the model with MPR as primary predictor (Table 28), asthma-only patients experienced $122 \%$ fewer outpatient visits than patients with both asthma and COPD. Being female was associated with an $8 \%$ increase in the number of outpatient visits, as compared to males. The number of outpatient visits increased with age and CMS. A $100 \%$ increase in medication adherence was associated with a $168 \%$ increase in the number of outpatient visits. When MPR was replaced by median gap (Table 29), every unit increase in median gap was associated with a $0.06 \%$ decrease in the number of outpatient visits. 
Table 28: Negative Binomial Regression Model with Predictors of Number of Outpatient Visits using MPR Adherence for the Entire Phase I Sample

\begin{tabular}{lccccc}
\hline Predictor & $\mathbf{e}^{\mathrm{x}}$ & Estimate & SE & Chi-Square & Significance \\
& & & & Statistic & \\
\hline Age & 1.0125 & 0.0125 & 0.0010 & 145.21 & $0.0001^{*}$ \\
Female Sex & 1.0878 & 0.0842 & 0.0201 & 17.59 & $0.0001^{*}$ \\
TMS & -- & 0.0005 & 0.0010 & 0.27 & 0.6018 \\
CMS & 1.0168 & 0.0167 & 0.0031 & 29.87 & $0.0001^{*}$ \\
COPD Dummy & -- & 0.0215 & 0.0273 & 0.62 & 0.4316 \\
Both Dummy & 2.2219 & -0.7984 & 0.0347 & 528.80 & $0.0001^{*}$ \\
MPR & 2.6815 & 0.9864 & 0.0320 & 949.46 & $0.0001^{*}$ \\
\hline
\end{tabular}

\footnotetext{
*Significant at $\mathrm{p}<=0.05$; SE: Standard Error; $\mathrm{e}^{\mathrm{x}}$ : Exponential of the estimate; Log Likelihood $=12268.55 ;$ Deviance $=0.8098, \mathrm{~N}=9,594 ;$ Chi-square $=20494.5 ;$ Sig. $=$ 0.000
} 
Table 29: Negative Binomial Regression Model with Predictors of Number of Outpatient Visits using Median Gap Adherence for the Entire Phase I Sample

\begin{tabular}{lccccc}
\hline Predictor & $\mathbf{e}^{\mathbf{x}}$ & Estimate & SE & Chi-Square & Significance \\
& & & & Statistic & \\
\hline Age & 1.010 & 0.0139 & 0.0011 & 175.15 & $0.0001^{*}$ \\
Female Sex & 1.070 & 0.0683 & 0.0204 & 11.27 & $0.0008^{*}$ \\
TMS & 1.002 & -0.0026 & 0.0010 & 6.79 & $0.0092^{*}$ \\
CMS & 1.030 & 0.0330 & 0.0031 & 116.21 & $0.0001^{*}$ \\
COPD Dummy & 1.080 & 0.0794 & 0.0277 & 8.20 & $0.0042^{*}$ \\
Both Dummy & 2.230 & -0.8027 & 0.0352 & 519.38 & $0.0001^{*}$ \\
Median Gap & 1.0006 & -0.0006 & 0.0000 & 518.64 & $0.0001^{*}$ \\
\hline
\end{tabular}

\footnotetext{
*Significant at $\mathrm{p}<=0.05$; SE: Standard Error; $\mathrm{e}^{\mathrm{x}}$ : Exponential of the estimate; Log Likelihood $=12046.72 ;$ Deviance $=0.82, \mathrm{~N}=9,594 ;$ Chi-square $=21850.69 ;$ Sig. $=$ 0.000
} 
The results of the OLS regression models measuring the impact of MPR adherence and median gap adherence on LOS are presented in Table 30 and Table 31, respectively. Both models were checked to see if the assumptions of OLS regression were met. The VIF was less than 10 for both models, indicating absence of multicollinearity. The Durbin-Watson statistic was around 1.7 for both models, indicating absence of autocorrelation. The plot of predicted versus residual values showed a uniform distribution, indicating absence of heteroskedasticity in both models. MPR adherence was a significant predictor of LOS. A $100 \%$ increase in adherence (since MPR adherence is measured on a scale on 0 to 1 ) was associated with an increase in LOS by 1.4 days. No other predictor variables were significant predictors of LOS. In the model using median gap as primary predictor, there were no significant predictors of LOS. 
Table 30: OLS Regression Model with Predictors of LOS using MPR Adherence for the Entire Phase I Sample

\begin{tabular}{lcccc}
\hline Predictor & Estimate & SE & T-statistic & Significance \\
\hline Age & 0.006 & 0.018 & 0.306 & 0.760 \\
Male Sex & -0.314 & 0.316 & -0.994 & 0.322 \\
TMS & -0.005 & 0.011 & -0.439 & 0.662 \\
CMS & -0.012 & 0.037 & -0.342 & 0.733 \\
COPD Dummy & -0.034 & 0.446 & -0.077 & 0.939 \\
Both Dummy & 0.057 & 0.457 & 0.126 & 0.900 \\
MPR & 1.405 & 0.383 & 3.671 & $0.000 *$ \\
\hline
\end{tabular}

*Significant at $\mathrm{p}<=0.05$; SE: Standard Error; Adjusted R-sq $=4.3 \%, \mathrm{~N}=181 ; \mathrm{F}=2.149$;

Sig. $=0.041$ 
Table 31: OLS Regression Model with Predictors of LOS using Median Gap Adherence for the Entire Phase I Sample

\begin{tabular}{lcccc}
\hline Predictor & Estimate & SE & T-statistic & Significance \\
\hline Age & 0.012 & 0.019 & 0.624 & 0.534 \\
Male Sex & -0.201 & 0.327 & -0.615 & 0.539 \\
TMS & -0.006 & 0.012 & -0.480 & 0.632 \\
CMS & 0.012 & 0.038 & 0.330 & 0.741 \\
COPD Dummy & 0.008 & 0.463 & 0.017 & 0.987 \\
Both Dummy & 0.248 & 0.472 & 0.525 & 0.600 \\
Median Gap & -0.001 & 0.000 & -0.392 & 0.695 \\
$\begin{array}{l}\text { *Significant at } \mathrm{p}<=0.05 ; \text { SE: Standard Error; Adjusted R-sq }=3.1 \%, \mathrm{~N}=181 ; \mathrm{F}=0.230 ; \\
\text { Sig. }=0.978\end{array}$ &
\end{tabular}




\section{Discussion for Research Question 4}

There was a significant variation in the number of adverse outcomes (hospitalizations or ED visits) by medication adherence for the COPD-only group, however, no significant differences were observed for the asthma-only and the both groups by medication adherence. Hence, the null hypothesis B is accepted for the asthma-only and the both groups. For the COPD-only group, null hypothesis B is rejected. Null hypothesis $\mathrm{C}$ is rejected for each of the 3 disease groups since the number of outpatient visits showed differences by medication adherence for each of the 3 disease groups. Since LOS showed differences by MPR, null hypothesis D is rejected.

The other variables that were able to explain differences in the number of adverse outcomes were sex, TMS, and disease severity (dummy codes). As expected, the number of adverse outcomes as well as the number of outpatient visits increased with disease severity. Patients having both asthma as well as COPD were more severe than those with COPD-only, or asthma only, and therefore experienced the highest number of adverse outcomes. Outpatient use too, was highest in the both group, and lowest in the asthma only group, which could be attributed to disease severity. TMS is also a proxy for disease severity, hence an increase in TMS was associated with an increase in the number of adverse outcomes.

Surprisingly, for the COPD-only group, the number of adverse outcomes increased with MPR. This can be explained by the fact that MPR indicates increased frequency of taking medications, which is related to disease severity. Similar results have been found by Balkrishnan et al (2000), who examined the association between medication adherence and number of adverse outcomes in the elderly. 
The other interesting finding is the difference in the number of adverse outcomes and outpatient use by gender. Females experienced significantly fewer adverse outcomes, but had higher outpatient utilization after adjusting for disease severity as well as medication adherence. Findings from the CDC Asthma Prevalence Survey have shown that outpatient utilization rates are significantly higher among females than males (CDC, 2001). The rate of hospitalizations and emergency room visits were also higher in females (CDC, 2001). Females tend to be more aware of their overall health and generally exhibit higher outpatient use than males, which could help potentially explain the differential use of healthcare services between males and females (Lindamer et al, 2003).

For outpatient use, an increase in the MPR was associated with increasing outpatient use. Conceptually, better medication adherence should result in improved outcomes and reduced healthcare utilization. However, the probability of receiving a prescription is higher after an outpatient visit (Joshi and Shireman, 2001). Thus, patients who took their medication more regularly visited their physician more often in order to obtain their prescription (since a prescription is generally a result of an outpatient visit).

There was a significant variation in the LOS by MPR in the combined model. Although LOS increased with MPR, the model had low predictive power $(\mathrm{R}-\mathrm{sq}=4.3 \%)$. Conceptually, the most important predictor of LOS is DRG-based reimbursement. Since PEIA bases its reimbursement on Medicare DRG rates, hospitals would try to reduce LOS according to the fixed sum they would receive based on the DRG. This is further reinforced by the fact that more than $90 \%$ of the hospitalizations had the LOS within two standard deviations of the mean (average $\operatorname{LOS}=3.9 \pm 1.9$ days; Range $=2-12$ days). 
Thus, LOS is more likely a function of DRG-based reimbursement, rather than medication adherence, which could help explain the low predictive power of the OLS regression models for LOS.

\section{Results for Research Question 5}

Objective 5 was to examine the impact of medication adherence on total healthcare costs (hospital, ED, outpatient, and prescription). OLS regression analysis was used to achieve this objective. Costs were log-transformed in order to achieve normality. For each of the OLS models using either MPR or median gap as primary predictors, each of the regression assumptions were met. Normality was achieved by logtransformation of total costs. The VIF was less than 10, indicating absence of multicollinearity. The Durbin-Watson statistic for each of the variables was around 2, indicating absence of autocorrelation. The graph of predicted versus residual values showed an even distribution, indicating absence of heteroskedasticity.

For the asthma-only group, TMS, asthma severity, and MPR were significant predictors of the total healthcare costs (Table 32). Every unit increase in TMS was associated with a $0.6 \%$ increase in total healthcare costs. Every unit increase in the level of asthma severity increased total healthcare costs by $11.7 \%$. An increase of $100 \%$ in medication adherence (as the MPR scores range from 0 to 1) was associated with a $241 \%$ increase in total healthcare costs. The variables together were able to explain $50 \%$ of the total variance in total healthcare costs. When MPR was replaced by median gap adherence as the primary predictor, the predictive power of the model dropped significantly to $30 \%$ from $50 \%$ (Table 33 ). As compared to the MPR model, the model 
using median gap had CMS, asthma severity, and median gap as significant predictors. Total costs increased by $19 \%$ with every unit increase in asthma severity, and by $3.7 \%$ with every unit increase in the number of chronic medications (CMS). Total costs decreased by $0.2 \%$ as adherence decreased (as indicated by a unit increase in median gap, which is one day). 
Table 32: OLS Regression Model with Predictors of Log-Transformed Total Healthcare Costs Using MPR Adherence for the Asthma-Only Group

\begin{tabular}{lcccc}
\hline Predictor & Estimate & SE & T-statistic & Significance \\
\hline Age & -0.004 & 0.003 & -1.549 & 0.122 \\
Male Sex & -0.09 & 0.059 & -1.498 & 0.134 \\
TMS & $0.006^{*}$ & 0.003 & 1.976 & $0.048^{*}$ \\
CMS & 0.005 & 0.009 & 0.518 & 0.604 \\
Asthma Severity & $0.117^{*}$ & 0.075 & 32.186 & $0.000^{*}$ \\
MPR & $0.002^{*}$ & 0.021 & 5.606 & $0.000^{*}$ \\
\hline
\end{tabular}

*Significant at $\mathrm{p}<=0.05 ;$ SE: Standard Error; Adjusted R-sq $=50.1 \%, \mathrm{~N}=1,400 ; \mathrm{F}=$ 235.028, Sig. $=0.000$ 
Table 33: OLS Regression Model with Predictors of Log-Transformed Total Healthcare Costs Using Median Gap Adherence for the Asthma-Only Group

\begin{tabular}{lcccc}
\hline Predictor & Estimate & SE & T-statistic & Significance \\
\hline Age & -0.001 & 0.003 & -0.104 & 0.917 \\
Male Sex & -0.006 & 0.070 & -0.084 & 0.933 \\
TMS & -0.004 & 0.003 & -1.145 & 0.253 \\
CMS & 0.037 & 0.010 & 3.687 & $0.000^{*}$ \\
Asthma Severity & 0.186 & 0.025 & 7.594 & $0.000^{*}$ \\
Median Gap & -0.002 & 0.000 & -18.297 & $0.000^{*}$ \\
\hline
\end{tabular}

*Significant at $\mathrm{p}<=0.05$; SE: Standard Error; Adjusted R-sq $=29.8 \%, \mathrm{~N}=1,400 ; \mathrm{F}=$ 100.166, Sig. $=0.000$ 
For the COPD-only group, age, TMS, and MPR were significant predictors of total healthcare costs (Table 34). Every unit increase in age was associated with a $0.3 \%$ increase in total healthcare costs. Every unit increase in TMS was associated with a $0.9 \%$ increase in total healthcare costs. An increase of $100 \%$ in medication adherence was associated with a $373 \%$ increase in total healthcare costs. The variables together were able to explain $48 \%$ of the total variance in total healthcare costs. Replacing MPR adherence in the model with median gap adherence reduced the predictive power of the model to $36 \%$ (Table 35). In this model, age, CMS and median gap adherence were significant predictors of total costs. Every unit increase in age was associated with a $0.6 \%$ increase in total healthcare costs. Every unit increase in CMS was associated with a $3.7 \%$ increase in total healthcare costs. Total costs decreased by $0.2 \%$ as adherence decreased (as indicated by a unit increase in median gap, which is one day). 
Table 34: OLS Regression Model with Predictors of Log-Transformed Total Healthcare Costs Using MPR Adherence for the COPD-Only Group

\begin{tabular}{lcccc}
\hline Predictor & Estimate & SE & T-statistic & Significance \\
\hline Age & 0.003 & 0.001 & 2.561 & $0.010^{*}$ \\
Male Sex & 0.010 & 0.024 & 0.419 & 0.675 \\
TMS & 0.009 & 0.001 & 7.252 & $0.000^{*}$ \\
CMS & 0.003 & 0.004 & 0.559 & 0.576 \\
MPR & 3.730 & 0.053 & 70.624 & $0.000^{*}$ \\
\hline
\end{tabular}

*Significant at $\mathrm{p}<=0.05 ;$ SE: Standard Error; Adjusted R-sq $=48.2 \%, \mathrm{~N}=7,002 ; \mathrm{F}=$ 1301.562, Sig. $=0.000$ 
Table 35: OLS Regression Model with Predictors of Log-Transformed Total Healthcare Costs Using Median Gap Adherence for the COPD-Only Group

\begin{tabular}{lcccc}
\hline Predictor & Estimate & SE & T-statistic & Significance \\
\hline Age & 0.006 & 0.001 & 3.936 & $0.000^{*}$ \\
Male Sex & 0.018 & 0.027 & 0.659 & 0.510 \\
TMS & 0.001 & 0.001 & 0.104 & 0.917 \\
CMS & 0.037 & 0.004 & 8.320 & $0.000^{*}$ \\
Median Gap & -0.002 & 0.000 & -52.277 & $0.000^{*}$ \\
\hline
\end{tabular}

*Significant at $\mathrm{p}<=0.05$; SE: Standard Error; Adjusted R-sq $=36.1 \%, \mathrm{~N}=7,002 \mathrm{~F}=$ 793.394, Sig. $=0.000$ 
For the both group, TMS and MPR were significant predictors of total healthcare costs (Table 36). Every unit increase in TMS was associated with a $0.6 \%$ increase in total healthcare costs. An increase of $100 \%$ in medication adherence was associated with a 233\% increase in total healthcare costs. The variables together were able to explain almost $53 \%$ of the total variance in total healthcare costs. Replacing the MPR adherence with median gap adherence reduced the predictive power of the model to $30 \%$ (Table 37). Age, CMS, and median gap were significant predictors of total costs. Every unit increase in age increased total costs by $1.3 \%$, and every unit increase in CMS increased total costs by $3.8 \%$. Total costs decreased by $0.1 \%$ as adherence decreased (as indicated by a unit increase in median gap, which is one day). 
Table 36: OLS Regression Model with Predictors of Log-Transformed Total Healthcare Costs Using MPR Adherence for the Both Group

\begin{tabular}{lcccc}
\hline Predictor & Estimate & SE & T-statistic & Significance \\
\hline Age & 0.004 & 0.003 & 1.154 & 0.249 \\
Male Sex & -0.100 & 0.068 & -1.468 & 0.142 \\
TMS & 0.006 & 0.003 & 2.099 & $0.036^{*}$ \\
CMS & 0.008 & 0.009 & 0.942 & 0.346 \\
MPR & 2.331 & 0.081 & 28.655 & $0.000^{*}$ \\
\hline
\end{tabular}

*Significant at $\mathrm{p}<=0.05 ;$ SE: Standard Error; Adjusted R-sq $=52.8 \%, \mathrm{~N}=937 ; \mathrm{F}=$ 211.05, Sig. $=0.000$ 
Table 37: OLS Regression Model with Predictors of Log-Transformed Total Healthcare Costs Using Median Gap Adherence for the Both Group

\begin{tabular}{lcccc}
\hline Predictor & Estimate & SE & T-statistic & Significance \\
\hline Age & 0.013 & 0.004 & 3.056 & $0.002^{*}$ \\
Male Sex & 0.024 & 0.083 & 0.291 & 0.771 \\
TMS & 0.003 & 0.004 & 0.869 & 0.385 \\
CMS & 0.038 & 0.010 & 3.703 & $0.000^{*}$ \\
Median Gap & -0.001 & 0.000 & -15.858 & $0.000^{*}$ \\
\hline
\end{tabular}

*Significant at $\mathrm{p}<=0.05$; SE: Standard Error; Adjusted R-sq $=30.2 \%, \mathrm{~N}=937 ; \mathrm{F}=$ 81.909, Sig. $=0.000$ 
In order to adjust for any confounding by disease severity, the regression model was rerun for the entire Phase I sample with dummy codes for the COPD-only, and both groups, using asthma-only as the reference group (Table 38). Age, COPD dummy, both dummy, TMS, and MPR were significant predictors of log-transformed total healthcare costs. Every unit increase in age was associated with a $0.3 \%$ increase in total healthcare costs. As compared to respondents with asthma-only, respondents having COPD only experienced a decrease in total healthcare costs by $11.2 \%$, whereas respondents having both asthma and COPD experienced an increase in total healthcare costs by $91.5 \%$.

Every unit increase in the total number of medications (TMS) was associated with a $1.0 \%$ increase in total healthcare costs. Total costs increased with increasing MPR; an increase of $100 \%$ in medication adherence was associated with a $309 \%$ increase in total healthcare costs. All the variables were able to explain $57 \%$ of the variance in total healthcare costs. Replacing MPR adherence with median gap adherence reduced the predictive power of the model to $46 \%$ (Table 39). Age, CMS, COPD dummy, both dummy and median gap were significant predictors of total costs. Every unit increase in age increased total costs by $0.5 \%$, and every unit increase in CMS increased total costs by $3.6 \%$. As compared to respondents with asthma-only, respondents having COPD only experienced a decrease in total healthcare costs by $28.7 \%$, whereas respondents having both asthma and COPD experienced an increase in total healthcare costs by $100.1 \%$. Total costs decreased by $0.2 \%$ as adherence decreased (as indicated by a unit increase in median gap, which is one day). 
Table 38: OLS Regression Model with Predictors of Log-Transformed Total Healthcare Costs Using MPR Adherence for the Entire Phase I Sample

\begin{tabular}{lcccc}
\hline Predictor & Estimate & SE & T-statistic & Significance \\
\hline Age & 0.003 & 0.001 & 2.213 & $0.027^{*}$ \\
Male Sex & -0.010 & 0.021 & -0.443 & 0.658 \\
TMS & 0.010 & 0.001 & 9.186 & $0.000^{*}$ \\
CMS & 0.003 & 0.003 & 0.781 & 0.435 \\
COPD Dummy & -0.112 & 0.030 & -3.735 & $0.000^{*}$ \\
Both Dummy & 0.915 & 0.042 & 22.035 & $0.000^{*}$ \\
MPR & 3.089 & 0.039 & 80.209 & $0.000^{*}$ \\
\hline
\end{tabular}

*Significant at $\mathrm{p}<=0.05 ; \mathrm{SE}$ : Standard Error; Adjusted R-sq $=57.0 \%, \mathrm{~N}=9,418 ; \mathrm{F}=$ 1750.097, Sig. $=0.000$ 
Table 39: OLS Regression Model with Predictors of Log-Transformed Total Healthcare Costs Using Median Gap Adherence for the Entire Phase I Sample

\begin{tabular}{lcccc}
\hline Predictor & Estimate & SE & T-statistic & Significance \\
\hline Age & 0.005 & 0.001 & 4.078 & $0.000^{*}$ \\
Male Sex & 0.014 & 0.024 & 0.606 & 0.544 \\
TMS & 0.001 & 0.001 & 0.814 & 0.415 \\
CMS & 0.036 & 0.004 & 9.635 & $0.000^{*}$ \\
COPD Dummy & -0.287 & 0.033 & -8.600 & $0.000^{*}$ \\
Both Dummy & 1.001 & 0.046 & 21.647 & $0.000^{*}$ \\
Median Gap & -0.002 & 0.000 & -57.948 & $0.000^{*}$ \\
\hline
\end{tabular}

*Significant at $\mathrm{p}<=0.05 ; \mathrm{SE}$ : Standard Error; Adjusted R-sq $=46.1 \%, \mathrm{~N}=9,418 ; \mathrm{F}=$ 1149.427, Sig. $=0.000$ 


\section{Discussion for Research Question 5}

Based on the analysis, total asthma and COPD-related healthcare costs showed significant differences by medication adherence. Thus, the null hypothesis E that there is no difference in total costs between patients who are adherent to maintenance medications and those who are not is rejected for each of the disease groups.

The primary independent variable - medication adherence as indicated by the MPR and median gap, was a significant predictor of total healthcare costs in each of the models. However, contrary to theory, total healthcare costs increased as medication adherence increased. Thus, findings from this study do not support the popular notion that prophylactic drug therapy decreases total costs. Similar results have been found in other studies (Balkrishnan et al, 1998, and Balkrishnan and Christensen, 2000). These findings can be explained by the fact that disease severity was a potential confounder in the models. Thus, an increase in the number of prescription refills was an indicator of increasing disease severity. When the models were rerun using dummy codes for patients with COPD only, and patients with both asthma and COPD, as compared to asthma-only as the reference, patients with COPD-only experienced lower total healthcare costs than patients with asthma-only, and patients with both asthma and COPD experienced significantly higher total costs that patients with asthma-only. Another likely explanation for the statistical association between medication adherence and total costs is the fact that more than half of total costs were due to prescription use, and since medication adherence was measured using refill frequency, increasing refills were associated with increasing total costs. An increase in TMS or CMS indicates worse overall health status, and 
increase in total healthcare costs with increasing TMS/CMS was as expected. Total costs

have been shown to increase with TMS and CMS (Balkrishnan and Christensen, 2000).

\section{Results for Research Question 6}

The objective of this research question was to examine the relative risk of adverse health outcomes such as a hospitalization or an ED visit as a consequence of medication nonadherence. A case-control study design was used for the analysis. Recipients who experienced an adverse outcome were cases, and those who did not were controls. Upon examining the refill patterns of cases preceding the adverse outcome (for six months), and controls for a predefined period (first six months of the study period), a logistic regression model was used with a dichotomous dependent variable indicating any versus no adverse outcome. The predictors used were age, gender, TMS, CMS, and asthma severity. Medication adherence as measured by the MPR was the main predictor variable. The results of the logistic regression models for each of the three groups are presented in Tables $40-43$.

For the asthma-only group (Table 40), TMS significantly impacted the probability of experiencing an adverse outcome. As TMS increased, the probability of experiencing an adverse outcome increased. No other variables including MPR were significant predictors. 
Table 40: Adjusted Estimates of Odds Ratios Predicting the Risk of Any Adverse

Outcome as a Consequence of Adherence Using Logistic Regression Analyses for the Asthma-Only Group

\begin{tabular}{|c|c|c|c|c|}
\hline Predictor & & OR & Significance & $95 \%$ CI \\
\hline \multicolumn{5}{|l|}{ Age } \\
\hline & $18-30^{\dagger}$ & & & \\
\hline & $31-45$ & 0.75 & 0.529 & $0.306-1.836$ \\
\hline & $46-64$ & 0.62 & 0.272 & $0.266-1.554$ \\
\hline Male Sex & & 0.73 & 0.253 & $0.427-1.251$ \\
\hline TMS & & 1.03 & $0.008 *$ & $1.007-1.050$ \\
\hline CMS & & 0.94 & 0.111 & $0.871-1.014$ \\
\hline \multicolumn{5}{|c|}{ Asthma-Severity } \\
\hline \multicolumn{5}{|c|}{ Level $\mathrm{I}^{\dagger}$} \\
\hline & Level II & 3.71 & 0.100 & $0.778-17.650$ \\
\hline & Level III & 0.66 & 0.409 & $0.316-1.602$ \\
\hline & Level IV & 1.41 & 0.220 & $0.813-2.461$ \\
\hline & Level V & 1.32 & 0.615 & $0.441-3.991$ \\
\hline MPR & & 1.02 & 0.957 & $0.538-1.920$ \\
\hline
\end{tabular}

*Significant at $\mathrm{p}<=0.05$; OR: Odds Ratio; 95\% CI: 95\% Confidence Interval; $\mathrm{N}=79$

Cases and 1334 Controls; -2 Log Likelihood Ratio = 590.440; Chi-square = 18.734, Sig.

$=0.044 ; \mathrm{R}-\mathrm{sq}=3.8 \%$

${ }^{\dagger}$ Reference Group 
For the COPD-only group (Table 41), age group, sex, TMS, and MPR significantly impacted the probability of experiencing an adverse outcome. As compared to recipients in the $18-30$ age group, recipients between $46-64$ years were $39 \%$ less likely to experience an adverse outcome. Males were $38 \%$ more likely to experience an adverse outcome. The probability of experiencing an adverse outcome increased with TMS. As the MPR increased, the probability of experiencing an adverse outcome increased significantly. For the both group (Table 42), there was no significant predictors impacting the probability of an adverse outcome. 
Table 41: Adjusted Estimates of Odds Ratios Predicting the Risk of Any Adverse Outcome as a Consequence of Adherence Using Logistic Regression Analyses for the COPD-Only Group

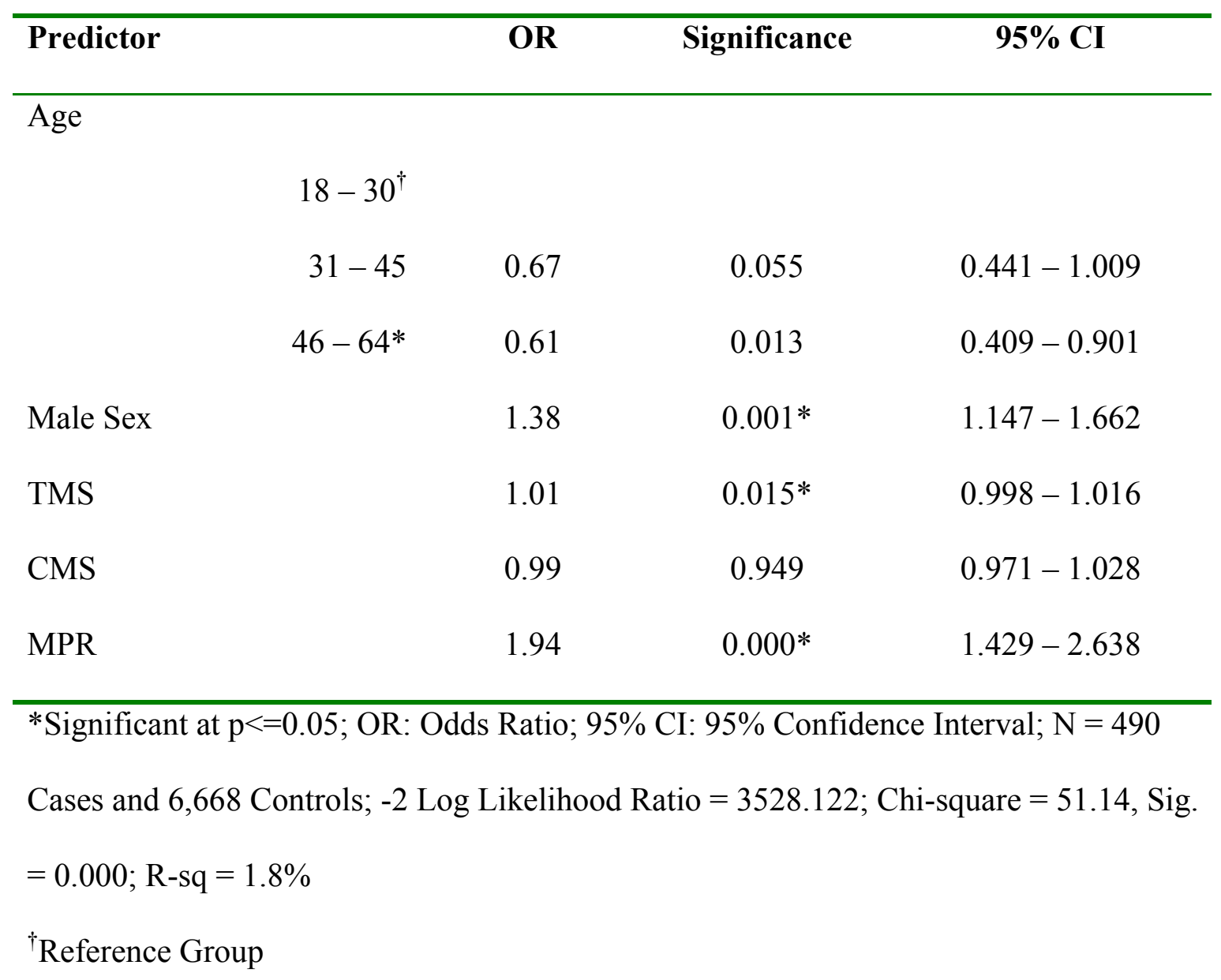


Table 42: Adjusted Estimates of Odds Ratios Predicting the Risk of Any Adverse Outcome as a Consequence of Adherence Using Logistic Regression Analyses for the Both Group

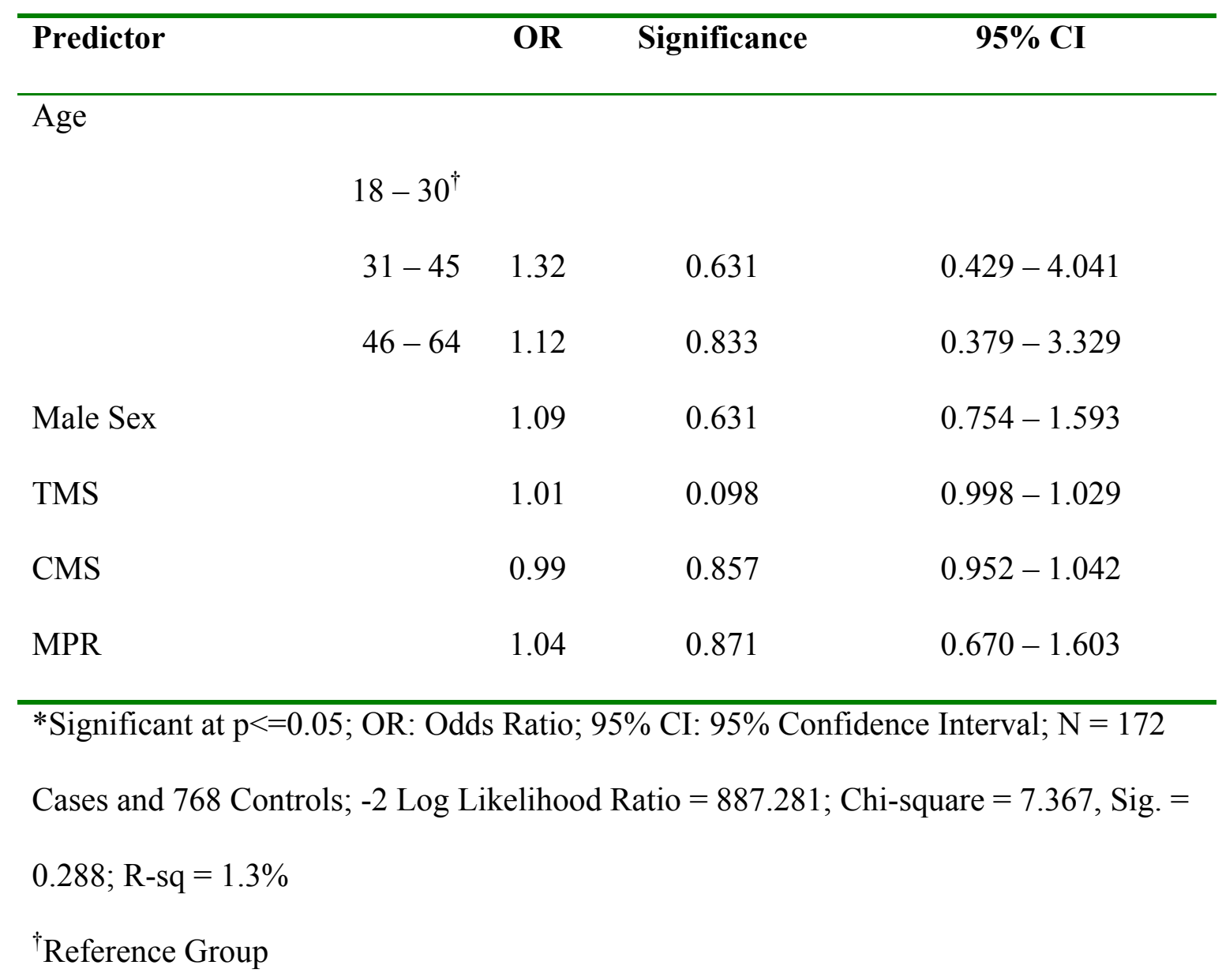


Since confounding by disease severity was a likely possibility, the model was rerun for the entire Phase I sample, with dummy variables for disease severity having asthma-only as the reference category (Table 43). Age group, sex, TMS, disease severity, and number of refills significantly impacted the probability of experiencing an adverse outcome. Recipients in the 46-64 age group were 35\% less likely to experience an adverse outcome as compared to enrollees in the $18-30$ year age group. Males were $25 \%$ more likely to experience an adverse outcome than females. The probability of experiencing an adverse outcome increased with TMS. Recipients with COPD-only were 1.36 times more likely to experience an adverse outcome than recipients with asthmaonly, and recipients with both asthma and COPD were 3.7 times more likely to experience an adverse outcome than recipients with asthma-only. As the MPR increased, the probability of experiencing an adverse outcome increased significantly. 
Table 43: Adjusted Estimates of Odds Ratios Predicting the Risk of Any Adverse Outcome as a Consequence of MPR Adherence Using Logistic Regression Analyses for the Entire Phase I Sample

\begin{tabular}{|c|c|c|c|}
\hline Predictor & OR & Significance & $95 \% \mathrm{CI}$ \\
\hline \multicolumn{4}{|l|}{ Age } \\
\hline $18-30^{\dagger}$ & & & \\
\hline $31-45$ & 0.74 & 0.101 & $0.521-1.059$ \\
\hline $46-64^{*}$ & 0.65 & 0.013 & $0.459-0.910$ \\
\hline Male Sex & 1.25 & $0.005^{*}$ & $1.053-1.445$ \\
\hline TMS & 1.01 & $0.002 *$ & $1.004-1.018$ \\
\hline CMS & 0.99 & 0.534 & $0.970-1.016$ \\
\hline \multicolumn{4}{|l|}{ Disease-Severity } \\
\hline \multicolumn{4}{|l|}{ Asthma Only ${ }^{\dagger}$} \\
\hline COPD Only & 1.36 & $0.015^{*}$ & $1.061-1.754$ \\
\hline Both Asthma and COPD & 3.71 & $0.000 *$ & $2.808-4.902$ \\
\hline MPR & 1.41 & $0.004 *$ & $1.117-1.790$ \\
\hline
\end{tabular}

\footnotetext{
*Significant at $\mathrm{p}<=0.05$; OR: Odds Ratio; 95\% CI: 95\% Confidence Interval; $\mathrm{N}=746$

Cases and 8,846 Controls; -2 Log Likelihood Ratio = 5062.75; Chi-square = 180.499, Sig. $=0.000 ;$ R-sq $=4.4 \%$

${ }^{\dagger}$ Reference Group
} 


\section{Discussion for Research Question 6}

Based on the analyses, the null hypothesis $\mathrm{F}$ that there is no significant difference in the risk of adverse outcomes between patients who adherent to maintenance medications and those who were not is accepted for the asthma-only, and the both groups. For the COPD-only group, the null hypothesis $\mathrm{F}$ is rejected.

Medication adherence as defined by the MPR prior to an adverse outcome for cases, and during a predefined period for controls was unable to significantly predict the probability of experiencing an adverse outcome for the asthma-only and the both groups. For the COPD-only group, increase in adherence was associated with an increased probability of experiencing an ED visit or a hospitalization. The inability of medication adherence to predict the risk of adverse outcomes could be attributed to omitted variable bias. Other factors that affect the risk of an ED visit or a hospitalization such as disease severity or exposure to specific risk factors such as smoke could not be included as predictors in the models. In the case of acute conditions such as asthma, acute attacks are not necessarily life-threatening and can be managed with use of quick-relief shortacting beta-agonists. A subanalysis showed that the mean number of maintenance medications used in the six months prior to an adverse outcome was 7 for cases, and 4 for controls in the entire Phase I sample. However, in the asthma-only group, the mean number of maintenance medications was 5.1 for cases, and 5.4 for controls. In the COPD-only group, the difference in mean number of prescriptions were significantly different in cases (5.0) versus controls (2.1), whereas the in both group, differences were not significant (13 in cases vs. 11 in controls). This probably explains why medication adherence was a significant predictor of adverse outcomes in the COPD-only and the 
combined group models. This was also reflected in the results of the combined model, where COPD-only patients, and patients with both asthma and COPD being more likely to experience adverse outcomes.

The other surprising finding was the reduced risk of adverse outcomes in the $46-$ 64 year age group, compared to the $18-30$ year age group, in the COPD-only and the both groups. Theoretically, older patients should be at a higher risk of experiencing adverse outcomes. It is possible that older patients are more knowledgeable about the disease, and are better at disease management, compared to younger patients, which resulted in fewer adverse outcomes requiring a visit to the hospital or the ER. The use of maintenance medications among 46 - 64 year old patients was three times higher than 18 - 30 year olds, which could on one hand, indicate greater disease severity, but on the other hand, it also indicates more experience in using inhaler medications. Males were significantly more likely to experience an adverse outcome than females. The mean number of maintenance medications used was similar in males and females. Studies have shown that the hospitalization rates as well as ED use rates were higher among asthmatic females than asthmatic males (Stempel et al, 1997 and CDC, 2001).

Overall, the findings from Phase I study objectives have shown that there was a relationship between medication nonadherence and related-healthcare utilization and costs. Instead of showing that medication adherence resulted in reduced healthcare utilization and costs, findings from Phase I have shown the opposite, most likely due to confounding by disease severity. This phase also identified the inability of claims data to recognize the severity levels of patients' conditions as a limitation for studying the utilization of healthcare services in patients with asthma and COPD. 


\section{Phase II Results}

Phase II of the study was designed to examine the impact of medication nonadherence on HRQL and productivity losses. HRQL was measured cross-sectionally using the St. George's Respiratory Questionnaire, which has been designed to measure HRQL in patients with both asthma as well as COPD (domains include symptoms, activity, and impact). The number of work-days lost due to respiratory illness and the number of unproductive hours while at work due to the disease were assessed using the Work Productivity Short Inventory (WPSI). Another objective of Phase II was to measure the self-reported nonadherence using the Morisky scale, and determine the congruence between self-reported adherence and adherence from claims data. All enrollees in the asthma-only and the both groups were mailed the questionnaire; whereas a random sample $(\mathrm{N}=2000)$ of COPD-only enrollees were mailed the questionnaire. Before analyzing the specific research questions, a response analysis was conducted. This helped us determine whether the respondents were representative of the general sample.

\section{Survey Response Analysis}

A total of 918 usable responses were obtained. This was after excluding 17 surveys due to incomplete data. This yielded an overall response rate of $22.6 \%$ with one mailing. Response rates for the asthma only group was the highest $(25.1 \%, \mathrm{~N}=385)$, followed by the both group $(24 \%, \mathrm{~N}=234)$, and was the lowest for the COPD-only group $(15 \%, N=299)$. As shown in Table 44 , more than $3 / 4^{\text {th }}$ of the respondents were in the 45 - 64 age group for all three disease groups. Also, as shown in Table 45, a majority of the respondents were female in each of the three disease groups. Chi-square statistics 
showed differences in age and gender distribution between the survey respondents and the overall Phase I sample for the asthma-only and the COPD-only groups. Overall, the age and gender distribution was similar between survey respondents, non-respondents, and the entire Phase I sample. 
Table 44: Number and Percent of Survey Respondents and Non-Respondents by Age Group

\begin{tabular}{|c|c|c|c|}
\hline $\begin{array}{l}\text { Age Range } \\
\text { (Years) }\end{array}$ & $\begin{array}{c}\text { Overall Sample } \\
\text { N (\%) }\end{array}$ & $\begin{array}{c}\text { Survey } \\
\text { Respondents } \\
\text { N (\%) }\end{array}$ & $\begin{array}{l}\text { Survey Non- } \\
\text { Respondents } \\
\text { N (\%) }\end{array}$ \\
\hline \multicolumn{4}{|l|}{ Asthma-only* } \\
\hline $18-30$ & $86(5.8)$ & $16(4.2)$ & $70(6.3)$ \\
\hline $31-45$ & $471(27.9)$ & $89(23.1)$ & $329(29.7)$ \\
\hline $45-64$ & $990(66.3)$ & $280(72.7)$ & $708(64.0)$ \\
\hline Total & $1,493(100.0)$ & $385(100.0)$ & $1,106(100.0)$ \\
\hline \multicolumn{4}{|l|}{ COPD-only* } \\
\hline $18-30$ & $322(4.5)$ & $11(3.7)$ & $77(4.5)$ \\
\hline $31-45$ & $1,862(26.0)$ & $53(17.7)$ & 465 (27.4) \\
\hline $45-64$ & $4,977(69.5)$ & $235(78.6)$ & $1,156(68.1)$ \\
\hline Total & $7,161(100.0)$ & $299(100.0)$ & $1,698(100.0)$ \\
\hline \multicolumn{4}{|c|}{ Both Asthma and } \\
\hline \multicolumn{4}{|l|}{$C O P D$} \\
\hline $18-30$ & $27(2.9)$ & $6(2.6)$ & $21(3.0)$ \\
\hline $31-45$ & $196(20.9)$ & $46(19.7)$ & $150(21.2)$ \\
\hline $45-64$ & $717(76.3)$ & $182(77.8)$ & $535(75.8)$ \\
\hline Total & 940 (100.) & $234(100.0)$ & $706(100)$. \\
\hline
\end{tabular}

*Significantly different $(\mathrm{p}<=0.05)$ 
Table 45: Number and Percent of Survey Respondents and Non-Respondents by Gender

\begin{tabular}{|c|c|c|c|}
\hline Gender & $\begin{array}{c}\text { Overall Sample } \\
\text { N (\%) }\end{array}$ & $\begin{array}{c}\text { Survey } \\
\text { Respondents } \\
\text { N (\%) }\end{array}$ & $\begin{array}{c}\text { Survey Non- } \\
\text { Respondents } \\
\text { N (\%) }\end{array}$ \\
\hline \multicolumn{4}{|c|}{ Asthma-only* } \\
\hline Male & $422(28.3)$ & $93(24.2)$ & 328 (29.7) \\
\hline Female & $1,071(71.7)$ & $292(75.8)$ & 778 (70.3) \\
\hline Total & $1,493(100.0)$ & $385(100.0)$ & $1,106(100.0)$ \\
\hline \multicolumn{4}{|c|}{ COPD-only* } \\
\hline Male & $2,719(38.0)$ & $99(33.1)$ & 656 (38.6) \\
\hline Female & $4,442(62.0)$ & $200(66.9)$ & $1,042(61.4)$ \\
\hline Total & $7,161(100.0)$ & $299(100.0)$ & $1,698(100.0)$ \\
\hline \multicolumn{4}{|c|}{ Both Asthma and } \\
\hline \multicolumn{4}{|l|}{$C O P D$} \\
\hline Male & $245(26.1)$ & $50(21.4)$ & 195 (27.6) \\
\hline Female & 695 (73.9) & 184 (78.6) & $511(72.4)$ \\
\hline Total & $940(100.0)$ & $234(100.0)$ & $706(100.0)$ \\
\hline
\end{tabular}

*Significantly different $(\mathrm{p}<=0.05)$ 


\section{Power Analysis}

For the asthma-only and the both groups, all patients as identified from Phase I were mailed the questionnaire. For the COPD-only group, 2000 patients were randomly selected and were mailed the questionnaire. In order to determine the representativeness of survey respondents as compared to the overall Phase I COPD population, a power analysis was carried out based on the proportion of COPD patients who were assumed to be adherent. Based on this power analysis, at least 384 usable responses have to be obtained in order to achieve representativeness (Kalton, 1987). Among the three disease groups, the COPD-only sample had the lowest response rate (15\%), with only 299 respondents out of the 2,000 who were mailed the survey sending usable responses. Thus, there is a possibility that this lack of power may potentially yield the COPD sample unrepresentative of the entire COPD population. The number of usable responses for the asthma-only group were 385 , with a response rate of $25.1 \%$, and the both group had 234 usable responses with a usable response rate of $24 \%$. In order to adjust for the lack of power in the COPD-only sample, the three groups were combined for analyzing each research question in Phase II, in addition to conducting specific analyses for each of the three groups. 
The demographic characteristics of the Phase II sample respondents are described in Table 46. The survey respondents were predominantly female with a mean age of 50.9 \pm 9 (SD) years. The average age was similar in the three disease groups. Overall, around $3 / 4^{\text {th }}$ of the total respondents were female. The gender distribution was similar for the asthma-only and the both groups (77\%), however, the COPD-only group had a relatively lower percentage of females. The respondents were very highly educated, with almost half the respondents having at least 4 years of college education. Only $25.3 \%$ of the respondents had less than a high school education, which was expected since PEIA enrollees are state employees, and are predominantly employed by state universities, or the state government.

Only $6.3 \%(\mathrm{~N}=58)$ of the respondents reported excellent health status, almost $27 \%$ reported very good, and $35 \%$ reported good health status. The proportion of respondents reporting excellent or very good health status decreased with increasing disease severity, with both group enrollees reporting the smallest proportion of enrollees with excellent or very good health status, followed by the COPD-only group, and the asthma-only group, in that order. 
Table 46: Demographic Characteristics of the Phase II Study Subjects

\begin{tabular}{|c|c|c|c|c|}
\hline Variable & Overall Sample & Asthma-only & COPD-only & Both \\
\hline Mean Age \pm SD (years) & $50.9 \pm 9.1$ & $50.1 \pm 9.3$ & $51.4 \pm 9.0$ & $51.7 \pm 8.7$ \\
\hline \multicolumn{5}{|l|}{ Gender* (N,\%) } \\
\hline Male & $232(25.1)$ & $87(22.7)$ & $93(31.7)$ & $51(22.2)$ \\
\hline Female & $677(73.3)$ & $296(77.3)$ & $200(68.3)$ & $178(77.4)$ \\
\hline \multicolumn{5}{|l|}{ Education* $(\mathrm{N}, \%)$} \\
\hline $8^{\text {th }}$ Grade or Less & $23(2.5)$ & $4(1.0)$ & $16(5.4)$ & $3(1.3)$ \\
\hline $9^{\text {th }}-12$ th Grade $/$ High & $210(22.8)$ & $83(21.7)$ & $71(24.1)$ & $56(23.9)$ \\
\hline \multicolumn{5}{|l|}{ School Graduate } \\
\hline College $1-3$ years & $232(25.1)$ & $83(21.7)$ & $86(29.2)$ & $61(26.1)$ \\
\hline College $>=4$ years & $450(48.8)$ & $212(55.5)$ & $122(41.4)$ & $113(48.3)$ \\
\hline \multicolumn{5}{|l|}{ Self-Reported Health } \\
\hline \multicolumn{5}{|l|}{ Status* (N, \%) } \\
\hline Excellent & $58(6.3)$ & $30(8.0)$ & $19(6.6)$ & $8(3.5)$ \\
\hline Very Good & $248(26.9)$ & $130(34.5)$ & $71(24.5)$ & $46(20.0)$ \\
\hline Good & $325(35.2)$ & $139(36.9)$ & $95(32.8)$ & $89(38.7)$ \\
\hline Fair & $192(20.8)$ & $67(17.8)$ & $67(23.1)$ & $58(25.2)$ \\
\hline Poor & $69(8.6)$ & $11(2.9)$ & $38(13.1)$ & $29(12.6)$ \\
\hline
\end{tabular}

*Significantly different $(\mathrm{p}<=0.05)$ 


\section{Results for Research Question 7}

The objective of research question 7 was to examine the overall HRQL among respondents with asthma and COPD, and compare differences in HRQL between the asthma-only, COPD-only, and both groups. The SGRQ was used to measure HRQL among the respondents, which yields a total HRQL score, as well as domain-specific scores for each of the three domains (symptoms, activity, and impacts). The SGRQ measured each score on a scale of 0 to 100 , where 0 indicated best HRQL, and 100 indicated worst HRQL. A reliability analysis was performed using Cronbach's alpha. The analysis showed that for the activity and impacts domains, Cronbach's alpha was 0.85 and 0.83 , respectively, indicating good internal consistency. For the symptoms domain, however, Cronbach's alpha was significantly lower than 0.7 , which is the cutoff for acceptable internal consistency. Similar problems with internal consistency for the symptoms domain of the SGRQ have been reported by Barr et al (2000), when they examined the psychometric stability of the SGRQ in the US population.

As shown in Table 47, the mean total score for the entire sample was 37.0 units. Overall HRQL was the worst for the Symptoms domain (57.0 units), followed by the Activity domain (43.6 units), and the Impacts domain (28.1 units). Mean HRQL scores obtained from this study sample were similar to those from other studies (Jones et al, 1992, Barr et al, 2000), thereby indicating a similar perception of HRQL among patients with asthma and COPD in the WV PEIA population. HRQL scores varied by age group, gender, self-reported health status and disease severity. As expected, HRQL worsened with age for each domain as well as the total score. Males reported statistically as well as 
clinically worse HRQL than females. A high correlation between self-reported health status and HRQL reinforced the validity of the SGRQ.

As expected, the total score as well as the domain-specific scores increased with increasing disease severity, with respondents in the both group having significantly worse HRQL (both statistically as well as clinically) as compared to the asthma-only and the COPD-only groups. Differences in scores between the asthma-only and COPD-only groups were neither statistically nor clinically significant (a difference of 4.0 units between groups is deemed clinically significant). 
Table 47: Health-Related Quality of life Scores for the Phase II Study Sample

\begin{tabular}{lcccc}
\hline Variable & Overall Sample & Asthma-only & COPD-only & Both \\
& & & \\
\hline HRQL Scores* (Mean & & & \\
\pm SD) & $37.0 \pm 22.0$ & 33.3 & 36.2 & 43.8 \\
Total Score & $53.1 \pm 23.1$ & 49.3 & 52.6 & 59.9 \\
Symptoms & $43.6 \pm 28.9$ & 39.6 & 41.7 & 52.5 \\
Activity & $28.1 \pm 21.3$ & 24.6 & 28.1 & 33.9 \\
Impacts & & & & \\
\hline
\end{tabular}

*Both group reported significantly worse HRQL scores than Asthma-only or COPD-

only; higher scores indicating worse HRQL $(p<=0.05)$ 


\section{Discussion for Research Question 7}

Mean domain-specific as well as total HRQL scores obtained from the WV PEIA sample were similar to those obtained in other patients with asthma and COPD (Hajiro et al, 1999, Jones et al, 1992, Barr et al, 2000, Spencer et al, 2000). In a study involving COPD patients with varying levels of disease severity, the total score, symptom score, activity score and impact score were 48 units, 66 units, 61 units, and 35 units, respectively (Spencer et al, 2000). In another study involving COPD patients in an outpatient setting, the mean total score, symptom score, activity score, and impacts score were 49 units, 42 units, 27 units, and 36 units, respectively (Hajiro et al, 1999). Similar perceptions of HRQL were observed in COPD patients in an outpatient setting (Domingo et al, 2002). Mean total score was 43.4 units, and the mean symptoms, activity, and impacts scores were 49,34, and 39 units, respectively (Domingo et al, 2002). Overall, the HRQL scores indicate a similar perception of HRQL and a similar impact of asthma and COPD on HRQL among WV PEIA members as compared to asthma and COPD patients in other parts of the United States.

As expected, HRQL deteriorated with increase in disease severity. The total score as well as domain-specific scores were significantly higher for PEIA members having both asthma and COPD, as compared to those who had COPD-only and asthma-only. The both group not only had statistically higher scores than asthma-only or COPD-only patients, but the scores were clinically significant as well ( $>4$ units). This is indicative of the validity of the SGRQ in measuring HRQL in patients with asthma and COPD. 


\section{Results for Research Question 8}

The objective of research question 8 was to examine the self-reported prevalence of nonadherence based on responses to the Morisky scale. The Morisky scale yields an overall adherence score on a scale of 0 to 4 , where 0 indicates most adherent, and 4 indicates least adherent. Patients with a score of ' 0 ' are classified as 'high adherent,' patients with scores between 1 and 2 are classified as 'medium adherent,' and those having scores between 3 and 4 are classified as 'low adherent.'

For the overall sample, $39.5 \%$ of the respondents were classified as high adherent, $14.4 \%$ of the respondents were medium adherent, and $40.4 \%$ were low adherent, based on their responses to the Morisky adherence scale (Table 48). Overall, the prevalence of adherence was similar in each of the three disease groups, although the chi-square tests showed that differences were statistically significant. The proportion of high adherent respondents was similar in the asthma-only and the COPD-only groups, and was slightly higher in the both group. Conversely, the proportion of medium adherent respondents was the lowest in the both group, and highest in the asthma-only group. The proportion of respondents having low adherence was the lowest in the COPD-only group, and were similar for the asthma-only and the both group, although the both group was slightly higher. 
Table 48: Self-Reported Adherence based on the Morisky Scale for the Phase II

\section{Study Sample}

\begin{tabular}{lcccc}
\hline $\begin{array}{l}\text { Morisky Adherence } \\
\text { Classification* }\end{array}$ & $\begin{array}{c}\text { Overall Sample } \\
\text { N (\%) }\end{array}$ & $\begin{array}{c}\text { Asthma-only } \\
\text { N (\%) }\end{array}$ & $\begin{array}{c}\text { COPD-only } \\
\text { N (\%) }\end{array}$ & $\begin{array}{c}\text { Both } \\
\text { N (\%) }\end{array}$ \\
\hline High Adherent* & $365(39.5)$ & $150(39.0)$ & $116(38.8)$ & $99(42.3)$ \\
Medium Adherent* & $133(14.4)$ & $62(16.1)$ & $39(13.0)$ & $29(12.4)$ \\
Low Adherent* & $373(40.4)$ & $162(42.1)$ & $105(35.1)$ & $104(44.4)$
\end{tabular}

*Distribution of adherence significantly different among the three disease groups

$(\mathrm{p}<=0.05$ ); Column-percentages do not total to $100 \%$ because responses to the Morisky adherence scale were missing for some respondents 


\section{Discussion for Research Question 8}

The prevalence of noandherence among asthma patients has been shown to be between 3-46\% (Bender, 2002, and Bauman et al 2002). On average, at least half of the patients with asthma are nonadherent to maintenance medications (Fish and Lung, 2001). Based on a self-report asthma-specific adherence scale, only $20 \%$ of patients with asthma were classified as adherent (Erickson et al, 2001). Therefore, we can say that the prevalence of adherence based on self-report, among PEIA members with asthma and COPD is similar to the overall prevalence of nonadherence among patients with asthma in the United States.

Conceptually, in the asthma-only group, the rate of medication adherence should be higher since guidelines explicitly recommend the use of prophylactic medications. Since patients having both asthma and COPD should be prescribed maintenance medications (based on asthma guidelines), one would expect the adherence rates to be higher in both of these groups as compared to the COPD-only group. Among PEIA enrollees with asthma, the proportion of adherent patients (high plus medium - 55\%) was slightly higher than those with COPD-only (52\%), and was similar to enrollees with both asthma and COPD (54\%). Ideally, the proportion of adherent COPD patients should be significantly lower than those with asthma, due to the absence of explicit guidelines recommending the use of maintenance medications unlike those for asthma.

It has been shown that patients with asthma tend to over-report adherence with self-report measures (Rand et al, 1992). Thus, adherence rates obtained from the Morisky scale could potentially be an overestimation of the true adherence in WV PEIA members with asthma and COPD. 


\section{Results for Research Question 9}

The objective of this question was to examine the congruence between selfreported adherence and refill-based adherence (MPR and median gap) for respondents having an MPR greater than zero. Spearman's correlation coefficient was used to measure the correlation. As shown in Table 49, there was a statistically significant correlation between median gap and MPR (as expected), and Morisky adherence was significantly correlated with both MPR and median gap, for the entire Phase II sample. The correlation between MPR and median gap was expected, since both measures was based on refill patterns from claims data. The negative sign exists because for the MPR, the higher the score (closer to 1.00) the higher the adherence, whereas for the median gap, the higher the score the lower the adherence (since gaps between refills would be larger). The same holds true for the relationship between Morisky scale adherence and MPR, since a higher score on the Morisky scale indicates low adherence. Although the correlation between Morisky scale and refill-based measures (MPR and median gap) is significant, the coefficient is relatively small, thus the statistical significance could be a function of sample size. 
Table 49: Spearman's Correlation between Self-Reported and Claims-Data Based Adherence for the Entire Phase II Sample

\begin{tabular}{lccc}
\hline Adherence & MPR & Morisky & Median Gap \\
Measure & & & \\
\hline MPR & 1.000 & $-0.193^{*}$ & $-0.309^{*}$ \\
Morisky & -- & 1.000 & $0.134^{*}$ \\
Median Gap & -- & -- & 1.000 \\
\hline
\end{tabular}

*Significant at $\mathrm{p}<0.01 ; \mathrm{N}=622$ 
Table 50 shows the correlations between the adherence measures for the asthmaonly group. A significant correlation was observed between MPR and median gap, and MPR and Morisky adherence. For the COPD-only group too (Table 51), a statistically significant correlation was found between the Morisky scale and MPR, but the magnitude of the correlation was small. No significant correlation was found between Morisky adherence and median gap. For respondents with both asthma and COPD, a significant correlation was found between self-reported adherence and each of the refill-based measures (Table 52). Although the correlation between MPR and Morisky scale, and median gap and Morisky scale was weak, it was statistically significant. The correlation between MPR and Median Gap was strong, as indicated by the correlation coefficient. 
Table 50: Spearman's Correlation between Self-Reported and Claims-Data Based Adherence for the Asthma-Only Group

\begin{tabular}{lccc}
\hline Adherence Measure & MPR & Morisky & Median Gap \\
\hline MPR & 1.000 & $-0.155^{*}$ & $-0.379^{*}$ \\
Morisky & -- & 1.000 & 0.114 \\
Median Gap & -- & -- & 1.000 \\
\hline
\end{tabular}

*Significant at $\mathrm{p}<0.01 ; \mathrm{N}=294$ 
Table 51: Spearman's Correlation between Self-Reported and Claims-Data Based Adherence for the COPD-Only Group

\begin{tabular}{lccc}
\hline Adherence Measure & MPR & Morisky & Median Gap \\
\hline MPR & 1.000 & $-0.287^{*}$ & -0.015 \\
Morisky & -- & 1.000 & 0.064 \\
Median Gap & -- & -- & 1.000 \\
\hline
\end{tabular}

*Significant at $\mathrm{p}<0.01 ; \mathrm{N}=127$ 
Table 52: Spearman's Correlation between Self-Reported and Claims-Data Based Adherence for the Both Group

\begin{tabular}{lccc}
\hline Adherence Measure & MPR & Morisky & Median Gap \\
\hline MPR & 1.000 & $-0.212^{*}$ & $-0.444^{*}$ \\
Morisky & -- & 1.000 & $0.239^{*}$ \\
Median Gap & -- & -- & 1.000 \\
\hline
\end{tabular}

*Significant at $\mathrm{p}<0.01 ; \mathrm{N}=198$ 


\section{Discussion for Research Question 9}

The objective of research question 9 was to examine the congruence between selfreported adherence and adherence obtained from claims data. Based on the analyses, for each of the three disease groups, as well as for the overall sample, there was no clinically significant correlation between self-reported adherence (Morisky scale) and adherence obtained from claims data (MPR or median gap). Thus, null hypothesis $\mathrm{G}$ is accepted for each of the three disease groups.

Although the Morisky scale was significantly correlated with MPR and median gap adherence, the magnitude of the correlation was not large enough to assume clinical significance. Statistical significance is simply a function of large sample size.

Although no significant correlations were found between self-reported and claims based measures of adherence in this study, both methods are widely used to measure adherence. Self-reports are the most easy and least equipment-intrusive method to measure adherence. These measures have been found to be valid measures of medication adherence (Brooks et al, 1994). Claims data based indices such as the MPR have also been shown to be valid measures of adherence (Steiner and Prochazka, 1997). It has been shown that in self-reports, respondents tend to over-report adherence (Rand et al, 1995). Using self-reports to measure adherence is also subject to acquiescence bias (Erickson et al, 2001), which suggests that a patient is more likely to respond positively to a question, regardless to the question asked. Either deliberate over-reporting of adherence or acquiescence bias could potentially explain the lack of correlation between Morisky adherence and MPR. 
Another possible explanation for the lack of correlation between the two measures in this study is the fact that self-report adherence was measured using a generic scale, as opposed to an asthma-specific scale. Although the Morisky scale has been shown to be a valid measure of adherence, an asthma-specific instrument would probably be able to better capture the intricacies of adherence to asthma-specific medications, such as being able to capture the correct/incorrect use of inhalers.

Among four studies that have compared adherence from claims data with selfreport measures, two studies found significant correlations between the two measures, while two did not (Steiner et al,1991, Wandless et al, 1979, and Peterson et al, 1982). Steiner et al (1991) examined the congruence between refill compliance for antihypertensive medications and self-report compliance using the Morisky scale, and found that patients reporting as noncompliant based on the Morisky scale correlated well with refill scores, however, among those reporting themselves as compliant, very poor correlations were found between the two measures (Steiner et al, 1991). Two other studies found significant correlations between refill-based and self-report adherence measures (Wandless et al, 1979, and Peterson et al, 1982).

\section{$\underline{\text { Results for Research Question } 10}$}

The objective of this question was to examine differences in HRQL by medication adherence. Separate OLS regression models were run for each group, with age, gender, education, self-reported health status, TMS, CMS, and adherence as predictors. Separate models were run with MPR as primary predictor, and with Morisky adherence as primary predictor. Asthma severity based on medication use was also included as a predictor for 
the asthma-only group model. The regression models were checked to see if the regression assumptions were met. The VIF was less than 10, indicating absence of multicollinearity. The Durbin-Watson statistic for each of the variables was around 2, indicating absence of autocorrelation. The graph of predicted versus residual values showed an even distribution, indicating absence of heteroskedasticity.

Table 53 shows the results of the OLS regression model identifying factors that successfully explained the variance in the total HRQL score for the asthma-only group. Results of the OLS models for the COPD-only and the both groups are presented in Tables 54 and 55, respectively.

For the asthma-only group (Table 53), self-reported health status and asthmaseverity, as determined from the index by Grana et al (1997) were significant predictors of the Total HRQL score. As self-reported health status worsened, the Total HRQL score increased, thereby indicating a deteriorating HRQL. The health status variable was included as a continuous variable on a scale of 1 to 5 , where 1 indicated excellent health status, and 5 indicated poor health status. With every unit increase in health status score, Total HRQL score increased by 13.7 units. Based on the asthma severity indicator, every unit increase in the level of asthma severity was associated with a 1.74 unit increase in Total HRQL score. Thus HRQL worsened with asthma severity. The variables together were able to explain $47.6 \%$ of the total variance in Total HRQL score, which indicates a high predictive power of the model. 
Table 53: OLS Regression Model with Predictors of Total HRQL Score Using MPR Adherence for the Asthma-Only Group

\begin{tabular}{lcccc}
\hline Predictor & Estimate & SE & T-statistic & Significance \\
\hline Age & -0.049 & 0.084 & -0.553 & 0.581 \\
Male Sex & -2.966 & 1.782 & -1.664 & 0.097 \\
Education & -0.773 & 0.927 & -0.843 & 0.405 \\
Health Status & 13.703 & 0.858 & 15.965 & $0.000^{*}$ \\
TMS & 0.008 & 0.091 & 0.088 & 0.930 \\
CMS & 0.157 & 0.252 & 0.623 & 0.534 \\
Asthma Severity & 1.743 & 0.583 & 2.988 & $0.003^{*}$ \\
MPR & -2.135 & 2.092 & -1.020 & 0.308 \\
\hline
\end{tabular}

*Significant at $\mathrm{p}<=0.05$; SE: Standard Error; Adjusted R-sq $=47.6 \%, \mathrm{~N}=359 ; \mathrm{F}=$ 41.794; Sig. $=0.000$ 
For the COPD-only group (Table 54), self-reported health status and MPR were significant predictors of total HRQL score. HRQL score worsened with worsening health status, and increasing medication adherence. With every unit increase in health status score, total HRQL score increased by 15.4 units. Total HRQL score increased with increasing MPR. The predictive power of the independent variables was even higher than the asthma-only model, since the variables together were able to explain $60.1 \%$ of the total variance in Total HRQL score. 
Table 54: OLS Regression Model with Predictors of Total HRQL Score Using MPR Adherence for the COPD-Only Group

\begin{tabular}{lcccc}
\hline Predictor & Estimate & SE & T-statistic & Significance \\
\hline Age & 0.107 & 0.112 & 0.951 & 0.342 \\
Male Sex & -0.238 & 2.056 & 0.116 & 0.908 \\
Education & -1.914 & 1.073 & -1.783 & 0.076 \\
Health Status & 15.369 & 0.990 & 15.519 & $0.000^{*}$ \\
TMS & 0.063 & 0.102 & 0.623 & 0.534 \\
CMS & -0.185 & 0.315 & -0.588 & 0.557 \\
MPR & 10.946 & 3.403 & 3.216 & $0.001^{*}$ \\
\hline
\end{tabular}

*Significant at $\mathrm{p}<=0.05$; SE: Standard Error; Adjusted R-sq $=60.1 \%, \mathrm{~N}=286 ; \mathrm{F}=$ 62.550; Sig. $=0.000$ 
For the both group (Table 55), education and self-reported health status were significant predictors of Total HRQL score. Education was also included as a continuous variable on a scale of 1 to 4 , where 1 indicated lowest education ( $8^{\text {th }}$ grade or less), and 4 indicated highest education (college $>=4$ years). Every unit increase in the level of education was associated with a 4-unit decrease in the Total score. Thus HRQL improved with increasing education. HRQL score worsened with worsening health status. With every unit increase in the health status score, Total HRQL score increased by 10.7 units. All the predictive variables were able to explain $43.4 \%$ of the variance in Total HRQL. 
Table 55: OLS Regression Model with Predictors of Total HRQL Score Using MPR Adherence for the Both Group

\begin{tabular}{lcccc}
\hline Predictor & Estimate & SE & T-statistic & Significance \\
\hline Age & 0.017 & 0.119 & 0.143 & 0.886 \\
Male Sex & 1.796 & 2.479 & 0.725 & 0.469 \\
Education & -3.886 & 1.225 & -3.171 & $0.002^{*}$ \\
Health Status & 10.698 & 1.067 & 10.022 & $0.000^{*}$ \\
TMS & 0.134 & 0.102 & 1.316 & 0.190 \\
CMS & 0.383 & 0.294 & 1.304 & 0.194 \\
MPR & -1.059 & 2.710 & -0.391 & 0.696 \\
\hline
\end{tabular}

*Significant at $\mathrm{p}<=0.05$; SE: Standard Error; Adjusted R-sq $=43.4 \%, \mathrm{~N}=229 ; \mathrm{F}=$ 26.114; Sig. $=0.000$ 
Since disease severity could be a potential confounder, the model was rerun for the entire sample of respondents, with a dummy codes for COPD-only, and both groups, with asthma-only as the reference group. The results of this model are shown in Table 56. Education, self-reported health status, and the dummy variable for the both group were significant predictors of Total HRQL. Every unit increase in the level of education was associated with a 2.2-unit decrease in the Total score. Thus HRQL improved with increasing education. HRQL score worsened with worsening health status. With every unit increase in the health status score, Total HRQL score increased by 13.6 units. As compared to the asthma-only group, respondents having both asthma and COPD experienced a 2.6-unit increase in Total HRQL score, thus indicating that HRQL worsened with disease severity. All the predictive variables were able to explain almost $52 \%$ of the variance in Total HRQL. 
Table 56: OLS Regression Model with Predictors of Total HRQL Score Using MPR Adherence for the Entire Phase II Sample

\begin{tabular}{lcccc}
\hline Predictor & Estimate & SE & T-statistic & Significance \\
\hline Age & 0.024 & 0.059 & 0.400 & 0.689 \\
Male Sex & -0.437 & 1.189 & -0.367 & 0.713 \\
Education & -2.216 & 0.614 & -3.606 & $0.000^{*}$ \\
Health Status & 13.625 & 0.554 & 24.614 & $0.000^{*}$ \\
TMS & 0.080 & 0.056 & 1.437 & 0.151 \\
CMS & 0.126 & 0.165 & 0.759 & 0.448 \\
COPD Dummy & -2.264 & 1.290 & -1.755 & 0.080 \\
Both Dummy & 2.605 & 1.311 & 1.987 & $0.047^{*}$ \\
MPR & 2.082 & 1.490 & 1.397 & 0.163 \\
\hline
\end{tabular}

*Significant at $\mathrm{p}<=0.05$; SE: Standard Error; Adjusted R-sq $=51.8 \%, \mathrm{~N}=890 ; \mathrm{F}=$ 107.182; Sig. $=0.000$ 
MPR was replaced by Morisky adherence as the predictor variable, and the models were rerun for each of the three disease groups as well as the combined model for the entire Phase II sample. The results of these models are presented in Tables $57-60$. In these models, dummy codes were used for medium adherence and low adherence, with high adherence as the reference group. The results were almost identical to the models using MPR adherence as a predictor, except for the fact that Morisky adherence was not a significant predictor in any of the models. All the other variables showed an identical impact as the models using MPR as a predictor. 
Table 57: OLS Regression Model with Predictors of Total HRQL Score Using Morisky Adherence for the Asthma-Only Group

\begin{tabular}{|c|c|c|c|c|}
\hline Predictor & Estimate & $\mathbf{S E}$ & T-statistic & Significance \\
\hline Age & -0.048 & 0.085 & -0.574 & 0.567 \\
\hline Male Sex & -3.081 & 1.782 & -1.729 & 0.085 \\
\hline Education & -0.725 & 0.930 & -0.780 & 0.436 \\
\hline Health Status & 13.727 & 0.860 & 15.957 & $0.000^{*}$ \\
\hline TMS & 0.017 & 0.091 & 0.188 & 0.851 \\
\hline CMS & 0.107 & 0.249 & 0.431 & 0.667 \\
\hline Asthma Severity & 1.604 & 0.566 & 0.118 & $0.005^{*}$ \\
\hline \multicolumn{5}{|l|}{ Morisky Scale } \\
\hline Medium Adherence & 1.240 & 1.653 & 0.750 & 0.454 \\
\hline Low Adherence & 2.331 & 2.237 & 1.042 & 0.298 \\
\hline
\end{tabular}


Table 58: OLS Regression Model with Predictors of Total HRQL Score Using Morisky Adherence for the COPD-Only Group

\begin{tabular}{|c|c|c|c|c|}
\hline Predictor & Estimate & SE & T-statistic & Significance \\
\hline Age & 0.169 & 0.113 & 1.493 & 0.137 \\
\hline Male Sex & 0.253 & 2.083 & 0.121 & 0.904 \\
\hline Education & -1.899 & 1.094 & -1.736 & 0.084 \\
\hline Health Status & 15.986 & 1.020 & 15.668 & $0.000^{*}$ \\
\hline TMS & 0.092 & 0.104 & 0.891 & 0.374 \\
\hline CMS & -0.142 & 0.325 & -0.438 & 0.661 \\
\hline \multicolumn{5}{|l|}{ Morisky Scale } \\
\hline Medium Adherence & -3.196 & 2.085 & -1.533 & 0.126 \\
\hline Low Adherence & -1.320 & 2.994 & -0.441 & 0.660 \\
\hline
\end{tabular}


Table 59: OLS Regression Model with Predictors of Total HRQL Score Using Morisky Adherence for the Both Group

\begin{tabular}{lcccc}
\hline Predictor & Estimate & SE & T-statistic & Significance \\
\hline Age & -0.017 & 0.121 & -0.149 & 0.882 \\
Male Sex & 2.098 & 2.499 & 0.839 & 0.402 \\
Education & -3.793 & 1.224 & -3.098 & $0.002^{*}$ \\
Health Status & 10.619 & 1.063 & 9.991 & $0.000^{*}$ \\
TMS & 0.123 & 0.102 & 1.209 & 0.228 \\
CMS & 0.363 & 0.290 & 1.250 & 0.213 \\
Morisky Scale & & & & 0.478 \\
Medium Adherence & -1.526 & 2.148 & -0.710 & 0.107 \\
\multicolumn{2}{l}{ Low Adherence } & -5.381 & 3.324 & -1.619 \\
*Significant at $\mathrm{p}<=0.05 ;$ SE: Standard Error; Adjusted R-sq $=43.8 \%, \mathrm{~N}=229 ; \mathrm{F}=$ \\
23.318; Sig. = 0.000
\end{tabular}


Table 60: OLS Regression Model with Predictors of Total HRQL Score Using Morisky Adherence for the Entire Phase II Sample

\begin{tabular}{|c|c|c|c|c|}
\hline Predictor & Estimate & SE & T-statistic & Significance \\
\hline Age & 0.027 & 0.059 & 0.468 & 0.640 \\
\hline Male Sex & -0.361 & 1.189 & -0.304 & 0.761 \\
\hline Education & -2.213 & 0.615 & -3.596 & $0.000 *$ \\
\hline Health Status & 13.676 & 0.556 & 24.584 & $0.000 *$ \\
\hline TMS & 0.084 & 0.056 & 1.499 & 0.134 \\
\hline CMS & 0.154 & 0.164 & 0.935 & 0.350 \\
\hline \multicolumn{5}{|l|}{ Disease Severity } \\
\hline COPD Dummy & -2.901 & 1.230 & -0.062 & $0.019 *$ \\
\hline Both Dummy & 2.683 & 1.312 & 0.053 & $0.041^{*}$ \\
\hline \multicolumn{5}{|l|}{ Morisky Scale } \\
\hline Medium Adherence & -0.723 & -0.723 & -0.016 & 0.518 \\
\hline Low Adherence & -0.122 & -0.122 & -0.002 & 0.938 \\
\hline
\end{tabular}




\section{Discussion for Research Question 10}

Based on the analysis, for the asthma-only and the both group, the null hypothesis $\mathrm{H}$ that there is no difference in HRQL between patients who are adherent to maintenance medications and those who are not is accepted. For the COPD-only group, the null hypothesis $\mathrm{H}$ is rejected since there was a difference in HRQL score by medication adherence.

The primary predictor variable of medication adherence, as indicated by the MPR, was not a significant predictor for the asthma-only and the both groups. This is not surprising since there is considerable endogeneity between medication adherence and HRQL. On one hand, patients who are adherent to medications should experience better outcomes consequently leading to an improved HRQL. However, disease severity can adversely impact HRQL, which in turn could result in reduced adherence to medication therapy. It has been shown that patients who are discouraged or depressed may be more likely to be noncompliant, contributing to a downward spiral of symptoms and exacerbations (Bosley et al, 1995). Conversely, it is also possible that patients who are worried about their disease may be more likely to be adherent to their medication regimen, but still report poor HRQL due to low levels of well-being (Schmeir and Leidy, 1998). The assessment of HRQL cross-sectionally in conjunction with medication adherence is difficult because of the endogeneity between adherence and HRQL. For the COPD-only model, MPR was associated with HRQL. As MPR increased, indicating better medication adherence, HRQL scores increased, indicating worsening of HRQL. The most likely explanation for this finding is that patients who consumed more medications were more severe, and had worse perceptions of HRQL. This hypothesis is 
further strengthened by the absence of explicit guidelines recommending the use of maintenance medications prophylactically in COPD. Thus, the MPR was inflated by an increased frequency of medication use due to disease severity, adversely impacting HRQL. In order to adjust to confounding by disease severity, a combined sample OLS model was run (Table 56). As shown in the model, patients having both asthma and COPD experienced significantly worse HRQL, than those having asthma-only, and COPD-only.

As expected, self-reported health status was a significant predictor of HRQL. In each of the three OLS models, as shown in Tables $53-55$, HRQL worsened with deteriorating perceived health status. Similar results have been found in other studies (Hajiro et al, 1999, Spencer et al, 2000). Spencer et al (2000) found that in patients with COPD, HRQL measured using the SGRQ worsened with deteriorating health status, which was also correlated with scores from the SF-36. The relationship between selfreported health status and HRQL also validates the measurement of HRQL using the SGRQ in WV PEIA recipients with asthma and COPD.

Education was a significant predictor among PEIA members with both asthma and COPD. With every unit increase in the level of education, the HRQL score decreased by a clinically significant 4 units. HRQL worsened as education increased for the combined sample model as well. Sociodemographic variables such as education have been shown to be significant predictors of HRQL in patients with respiratory illnesses. Educated individuals are more likely to be compliant as compared to those with lesser education (Tashkin, 1995). Although age and gender did not significantly impact HRQL in any of the above models, it has been shown that women with asthma or COPD are 
more likely to be compliant with maintenance medications than men (James et al, 1985). It has also been shown that older patients with asthma were more likely to be adherent than younger patients with asthma (Bailey et al, 1992) and had lower rates of healthcare utilization. Rand and colleagues (1995) reported that adherence was more likely to be higher among females who were married, and older, after adjusting for various covariates in a multivariate logistic regression model.

The OLS models could potentially suffer from omitted variable bias. Clinical measures, socioeconomic status, and the cost of medications are variables that can predict adherence, and subsequently impact HRQL. Absence of data on these variables could be a potential limitation as well.

\section{Results for Research Question 11}

Differences in productivity-related costs by disease group are shown in Table 61. The number of days experienced with the disease were higher for the COPD-only group and the both group as compared with the both group. The number of days missed from work increased with disease severity, with asthma-only patients experiencing an average of 5 lost workdays per year, and patients with both asthma and COPD experiencing almost 12 lost workdays per year. Although the number of unproductive hours while at work were similar among the three groups, this translated into significantly higher productivity losses for the COPD-only group, and the both group as compared with the asthma-only group. Total productivity losses increased with increasing disease severity as shown in Table 61. 
Table 61: Workplace Productivity Costs for the Phase II Study Sample

\begin{tabular}{lcccc}
\hline Variable & Overall Sample & Asthma-only & COPD-only & Both \\
& & & & \\
\hline Productivity & & & & \\
(Mean \pm SD) & $92.6 \pm 120.1$ & 76.0 & 100.7 & 112.6 \\
Days Experienced & $7.59 \pm 20.0$ & 5.3 & 7.0 & 11.9 \\
Days Lost & $1.3 \pm 2.2$ & 1.1 & 1.5 & 1.5 \\
Work-Hours & & & & 1338.3 \\
Unproductive & $852.1 \pm 2273.0$ & 596.9 & 786.2 & 1187.3 \\
Abseenteeism Dollars* & $925.1 \pm 2640.9$ & 657.7 & 1130.3 & 2461.7 \\
Presenteeism Dollars* & $1747.9 \pm 3758.1$ & 1232.5 & 1926.9 & \\
Total Dollars* & & & & \\
\hline
\end{tabular}

*Both group reported significantly higher productivity losses than Asthma-only or COPD-only $(\mathrm{p}<=0.05)$; higher scores indicating worse HRQL 
The objective of this question was to examine the impact of medication adherence on total productivity dollars (absenteeism plus presenteeism). OLS regression analysis was used to examine the impact of medication adherence on the dependent variable total productivity dollars. Log-transformed total productivity dollars were used in the OLS regression model in order to yield normality. The results of the semi-logarithmic model for each of the three disease groups are presented in Tables 62, 63 and 64. For each of the OLS models using either MPR or median gap as primary predictors, each of the regression assumptions were met. Normality was achieved by log-transformation of total productivity dollars. The VIF was less than 10, indicating absence of multicollinearity. The Durbin-Watson statistic for each of the variables was around 2, indicating absence of autocorrelation. The graph of predicted versus residual values showed an even distribution, indicating absence of heteroskedasticity.

For the asthma-only group (Table 62), age, self-reported health status, and TMS were significant predictors of the total productivity dollars. Every unit increase in age was associated with a $2.9 \%$ decrease in total productivity dollars. Self-reported health status was included as a continuous variable on a scale of 1 to 5 , where 1 indicated excellent health status, and 5 indicated poor health status. Every unit increase in selfreported health status was associated with a $74.5 \%$ increase in total productivity dollars lost. Thus, as self-reported health status worsened, total productivity dollars lost increased. Every unit increase in TMS was associated with a 2.9\% increase in total productivity dollars lost. The variables together were able to explain $30.1 \%$ of the total variance in total productivity dollars lost. 
Table 62: OLS Regression Model with Predictors of Log-Transformed Total Productivity Dollars Using MPR Adherence for the Asthma-Only Group

\begin{tabular}{lcccc}
\hline Predictor & Estimate & SE & T-statistic & Significance \\
\hline Age & -0.029 & 0.010 & -2.765 & $0.006^{*}$ \\
Male Sex & -0.351 & 0.227 & -1.548 & 0.123 \\
Education & 0.055 & 0.111 & 0.494 & 0.622 \\
Health Status & 0.745 & 0.106 & 7.059 & $0.000^{*}$ \\
TMS & 0.029 & 0.010 & 2.845 & $0.005^{*}$ \\
CMS & -0.051 & 0.028 & -1.806 & 0.073 \\
Asthma Severity & 0.064 & 0.069 & 0.924 & 0.357 \\
MPR & -0.141 & 0.258 & -0.546 & 0.586 \\
\hline
\end{tabular}

*Significant at $\mathrm{p}<=0.05$; SE: Standard Error; Adjusted R-sq $=30.1 \%, \mathrm{~N}=173 ; \mathrm{F}=$ 10.303, Sig. $=0.000$ 
For the COPD-only group (Table 63), male sex and self-reported health status were significant predictors of total productivity dollars lost. Males experienced $67.8 \%$ higher total productivity dollars lost than females, and every unit increase in self-reported health status was associated with an almost $40 \%$ increase in total productivity dollars lost. 
Table 63: OLS Regression Model with Predictors of Log-Transformed Total Productivity Dollars Using MPR Adherence for the COPD-Only Group

\begin{tabular}{lcccc}
\hline Predictor & Estimate & SE & T-statistic & Significance \\
\hline Age & -0.016 & 0.014 & -1.142 & 0.256 \\
Male Sex & 0.678 & 0.255 & 2.654 & $0.009^{*}$ \\
Education & -0.218 & 0.122 & -1.784 & 0.077 \\
Health Status & 0.397 & 0.119 & 3.341 & $0.001^{*}$ \\
TMS & 0.008 & 0.011 & 0.660 & 0.510 \\
CMS & -0.006 & 0.044 & -0.133 & 0.894 \\
MPR & -0.333 & 0.438 & -0.759 & 0.449 \\
\hline
\end{tabular}

*Significant at $\mathrm{p}<=0.05$; SE: Standard Error; Adjusted R-sq $=22.2 \%, \mathrm{~N}=121 ; \mathrm{F}=5.938$, Sig. $=0.000$ 
For the both group (Table 64), education and self-reported health status were significant predictors of total productivity dollars. Total productivity dollars lost decreased with increasing education, and increased with deteriorating health status. Every unit increase in the level of education was associated with a $38.4 \%$ decrease in total productivity dollars lost. Every unit increase in self-reported health status was associated with a $56.8 \%$ increase in total productivity dollars lost. MPR was not a significant predictor of total productivity dollars in either of the models. 
Table 64: OLS Regression Model with Predictors of Log-Transformed Total Productivity Dollars Using MPR Adherence for the Both Group

\begin{tabular}{lcccc}
\hline Predictor & Estimate & SE & T-statistic & Significance \\
\hline Age & -0.008 & 0.013 & -0.575 & 0.567 \\
Male Sex & -0.029 & 0.306 & -0.096 & 0.924 \\
Education & -0.384 & 0.133 & -2.894 & $0.005^{*}$ \\
Health Status & 0.568 & 0.128 & 4.431 & $0.000^{*}$ \\
TMS & 0.009 & 0.011 & 0.837 & 0.404 \\
CMS & -0.001 & 0.035 & -0.018 & 0.985 \\
MPR & 0.165 & 0.300 & 0.549 & 0.584 \\
*Significant at $\mathrm{p}<=0.05 ;$ SE: Standard Error; Adjusted R-sq $=22.6 \%, \mathrm{~N}=115 ; \mathrm{F}=5.808$, \\
Sig. $=0.000$
\end{tabular}


In order to adjust for any confounding by disease severity, the regression model was rerun for the entire sample of respondents with dummy codes for the COPD-only, and both groups, using asthma-only as the reference group. The results of this model are summarized in Table 65. Age, education, self-reported health status, TMS, and the dummy variable for the both group were significant predictors of log-transformed total productivity dollars lost. Contrary to expectation, every unit increase in age was associated with a $1.9 \%$ decrease in total productivity dollars lost. Total productivity dollars lost decreased with increasing education, and increased with deteriorating health status. Every unit increase in the level of education was associated with a $14.8 \%$ decrease in total productivity dollars lost. Every unit increase in self-reported health status (which indicates deteriorating health status) was associated with a $62.5 \%$ increase in total productivity dollars lost. Every unit increase in the total number of medications used (TMS) was associated with a $1.7 \%$ increase in total productivity dollars lost. As compared to respondents with asthma-only, respondents having both asthma and COPD experienced an increase in total productivity dollars lost by $29.2 \%$. All the variables were able to explain $25 \%$ of the variance in total productivity dollars lost. 
Table 65: OLS Regression Model with Predictors of Log-Transformed Total Productivity Dollars Using MPR Adherence for the Entire Phase II Sample

\begin{tabular}{lcccc}
\hline Predictor & Estimate & SE & T-statistic & Significance \\
\hline Age & -0.019 & 0.007 & -2.731 & $0.007^{*}$ \\
Male Sex & 0.034 & 0.144 & 0.236 & 0.813 \\
Education & -0.148 & 0.070 & -2.117 & $0.035^{*}$ \\
Health Status & 0.625 & 0.065 & 9.554 & $0.000^{*}$ \\
TMS & 0.017 & 0.006 & 2.867 & $0.004^{*}$ \\
CMS & -0.025 & 0.019 & -1.286 & 0.199 \\
COPD Dummy & 0.044 & 0.173 & 0.302 & 0.763 \\
Both Dummy & 0.292 & 0.147 & 2.052 & $0.041^{*}$ \\
MPR & -0.038 & 0.142 & -0.221 & 0.825 \\
\hline
\end{tabular}

*Significant at $\mathrm{p}<=0.05$; SE: Standard Error; Adjusted R-sq $=25.0 \%, \mathrm{~N}=417 ; \mathrm{F}=$ 16.408, Sig. $=0.000$ 
MPR was replaced by Morisky adherence as the predictor variable, and the models were rerun for each of the three disease groups as well as the combined model for the entire Phase II sample with log-transformed total productivity costs as the dependent variable. The results of these models are presented in Tables $66-69$. In these models, dummy codes were used for medium adherence and low adherence, with high adherence as the reference group. The results were almost identical to the models using MPR adherence as a predictor, and Morisky adherence was not a significant predictor in any of the models. All the other variables showed an identical impact as the models using MPR as a predictor. 
Table 66: OLS Regression Model with Predictors of Log-Transformed Total Productivity Dollars Using Morisky Adherence for the Asthma-Only Group

\begin{tabular}{|c|c|c|c|c|}
\hline Predictor & Estimate & SE & T-statistic & Significance \\
\hline Age & -0.028 & 0.010 & -2.706 & $0.008^{*}$ \\
\hline Male Sex & -0.353 & 0.227 & -1.554 & 0.122 \\
\hline Education & 0.049 & 0.111 & 0.439 & 0.662 \\
\hline Health Status & 0.745 & 0.106 & 7.038 & $0.000 *$ \\
\hline TMS & 0.029 & 0.010 & 2.855 & $0.005^{*}$ \\
\hline CMS & -0.052 & 0.028 & -1.865 & 0.064 \\
\hline Asthma Severity & 0.057 & 0.068 & 0.839 & 0.403 \\
\hline \multicolumn{5}{|l|}{ Morisky Scale } \\
\hline Medium Adherence & 0.015 & 0.197 & 0.075 & 0.941 \\
\hline Low Adherence & 0.184 & 0.267 & 0.688 & 0.492 \\
\hline
\end{tabular}


Table 67: OLS Regression Model with Predictors of Log-Transformed Total Productivity Dollars Using Morisky Adherence for the COPD-Only Group

\begin{tabular}{|c|c|c|c|c|}
\hline Predictor & Estimate & SE & T-statistic & Significance \\
\hline Age & -0.017 & 0.014 & -1.237 & 0.219 \\
\hline Male Sex & 0.634 & 0.255 & 2.486 & $0.014 *$ \\
\hline Education & -0.225 & 0.122 & -1.844 & 0.068 \\
\hline Health Status & 0.404 & 0.120 & 3.361 & $0.001 *$ \\
\hline TMS & 0.008 & 0.012 & 0.702 & 0.484 \\
\hline CMS & -0.011 & 0.045 & -0.239 & 0.812 \\
\hline \multicolumn{5}{|l|}{ Morisky Scale } \\
\hline Medium Adherence & -0.206 & 0.232 & -0.886 & 0.378 \\
\hline Low Adherence & -0.052 & 0.320 & -0.161 & 0.873 \\
\hline
\end{tabular}

*Significant at $\mathrm{p}<=0.05$; SE: Standard Error; Adjusted R-sq $=21.7 \%, \mathrm{~N}=121 ; \mathrm{F}=5.189$, Sig. $=0.000$ 
Table 68: OLS Regression Model with Predictors of Log-Transformed Total Productivity Dollars Using Morisky Adherence for the Both Group

\begin{tabular}{lcccc}
\hline Predictor & Estimate & SE & T-statistic & Significance \\
\hline Age & -0.009 & 0.014 & -0.723 & 0.471 \\
Male Sex & 0.049 & 0.305 & 0.163 & 0.871 \\
Education & -0.378 & 0.133 & -2.847 & $0.005^{*}$ \\
Health Status & 0.557 & 0.130 & 4.294 & $0.000^{*}$ \\
TMS & 0.009 & 0.012 & 0.808 & 0.421 \\
CMS & 0.001 & 0.034 & 0.035 & 0.972 \\
Morisky Scale & & & & \\
Medium Adherence & -0.075 & 0.237 & -0.318 & 0.751 \\
$\quad$ Low Adherence & -0.266 & 0.375 & -0.710 & 0.479 \\
\end{tabular}

*Significant at $\mathrm{p}<=0.05$; SE: Standard Error; Adjusted R-sq $=22.1 \%, \mathrm{~N}=115 ; \mathrm{F}=5.072$, Sig. $=0.000$ 
Table 69: OLS Regression Model with Predictors of Log-Transformed Total Productivity Dollars Using Morisky Adherence for the Entire Phase II Sample

\begin{tabular}{lcccc}
\hline Predictor & Estimate & SE & T-statistic & Significance \\
\hline Age & -0.019 & 0.007 & -2.731 & $0.007^{*}$ \\
Male Sex & 0.023 & 0.142 & 0.165 & 0.869 \\
Education & -0.149 & 0.070 & -2.135 & $0.033^{*}$ \\
Health Status & 0.629 & 0.066 & 9.591 & $0.000^{*}$ \\
TMS & 0.017 & 0.006 & 2.865 & $0.004^{*}$ \\
CMS & -0.025 & 0.019 & -1.331 & 0.184 \\
COPD Dummy & 0.040 & 0.140 & 0.288 & 0.773 \\
Both Dummy & 0.278 & 0.142 & 1.964 & $0.050^{*}$ \\
Morisky Scale & & & & \\
Medium Adherence & -0.120 & 0.126 & -0.954 & 0.341 \\
$\quad$ Low Adherence & -0.026 & 0.174 & -0.150 & 0.881 \\
\hline
\end{tabular}

*Significant at $\mathrm{p}<=0.05$; SE: Standard Error; Adjusted R-sq $=24.9 \%, \mathrm{~N}=417 ; \mathrm{F}=$ 14.855, Sig. $=0.000$ 


\section{Discussion for Research Question 11}

The impact of asthma and COPD on absenteeism and presenteeism was significantly lower in PEIA members as compared to other asthma and COPD patients with asthma and COPD in the United States. Findings from the World Health Organization's Work and Health Productivity Questionnaire have shown that asthma patients experienced 11 mean absenteeism days, and patients with COPD experienced 19 mean absenteeism days per year in the United States (Wang et al, 2003). Productivity dollars in PEIA COPD patients were slightly higher than national estimates. Halpern et al (2003) showed that mean productivity costs in patients with emphysema were $\$ 1,527$ per patient per year. In PEIA asthma patients, costs were slightly lower than other studies. Cisternas et al (2003) found that productivity costs in patients with asthma were $\$ 1,550$ per patient per year.

OLS regression was used to estimate the impact of medication adherence on total productivity. Based on the analysis, the null hypothesis I that there were no difference in workplace productivity dollars between those who were adherent to maintenance medications as compared to those who were not, is accepted for each of the three disease groups as well as the combined sample.

To date, no published study has examined the relationship between medication adherence and productivity-related outcomes. For each of the disease-specific models, medication adherence as indicated by the MPR was not a significant predictor of total productivity costs. As expected, self-reported health status was a significant predictor in each of the three models, and productivity losses increased as health status worsened. The inability of MPR to significantly predict productivity losses could be attributed to 
potential omitted variable bias. In this case, the omitted variable could most likely be disease severity. This was confirmed by the results of the combined model, which showed that patients with both asthma and COPD experienced significantly higher productivity losses than patients with asthma only. Confounding by disease severity was also indicated by the significance of the TMS variable. Productivity losses increased as the number of total medications increased, which is indicative of worse overall health status. Productivity losses in patients with COPD have been shown to increase with disease severity (Halpern et al, 2003). In asthmatic patients too, disease severity significantly increase productivity dollars. Patients with mild, moderate, and severe asthma experienced total per-person healthcare costs of $\$ 2,646, \$ 4,530$, and $\$ 12,813$, respectively, $35 \%$ of which were related to absenteeism and reduced effectiveness at work (Cisternas et al, 2003). In the COPD-only model, males experienced significantly higher productivity losses than females. These gender differences could be attributed to higher incomes among males than females (Jacobson et al, 2000), thus a lost workday or an unproductive work-hour translates into higher dollar values for males than females.

In the combined model, productivity losses decreased as the level of education increased, with productivity losses decreasing by $15 \%$ with every increasing level of eductation. It has been shown that COPD patients with more than high school education had $30 \%$ higher indirect costs as compared to those with less than high school education, and a significant proportion of which were attributed to productivity losses (Halpern et al, 2003). Thus, it can be assumed that educated patients tend to be more aware about disease management, and the deleterious effects of potential risk factors such as smoking, which results in worse outcomes. Indirect costs in COPD patients who smoked were 
significantly higher than those who did not (Halpern et al, 2003). Increase in tobacco use was associated with significant increases in lost productive time, and productivity dollars. Individuals who smoked greater than 1 pack of cigarettes per day, experienced $70 \%$ higher losses in productivity dollars than those who never smoked (Stewart et al, 2003). Thus, the absence of smoking as a predictor could potentially induce omitted variable bias.

The other surprising result was the relationship between age and productivity dollars. As age increased, productivity losses decreased. Similar results have been found in other studies (Stewart et al, 2003). The study showed that the cost equivalent of lost productive time was the highest for $40-49$ year olds, as compared to $50-65$ year-olds. A subanalysis was carried out examining the bivariate relationship between age, absenteeism, and presenteeism dollars. This subanalysis showed that although absenteeism dollars increased with age, presenteeism dollars were highest among recipients between 18 and 30 years of age (Figure 5). This could be explained by the fact that disease severity increased with age, which led to a higher number of days missed from work. Younger patients were less severe and were able to attend work, thereby explaining the higher productivity losses while at work as opposed to older patients who experienced greater absenteeism dollars. 
Figure 5: Bivariate Relationship between Productivity Dollars and Age Group

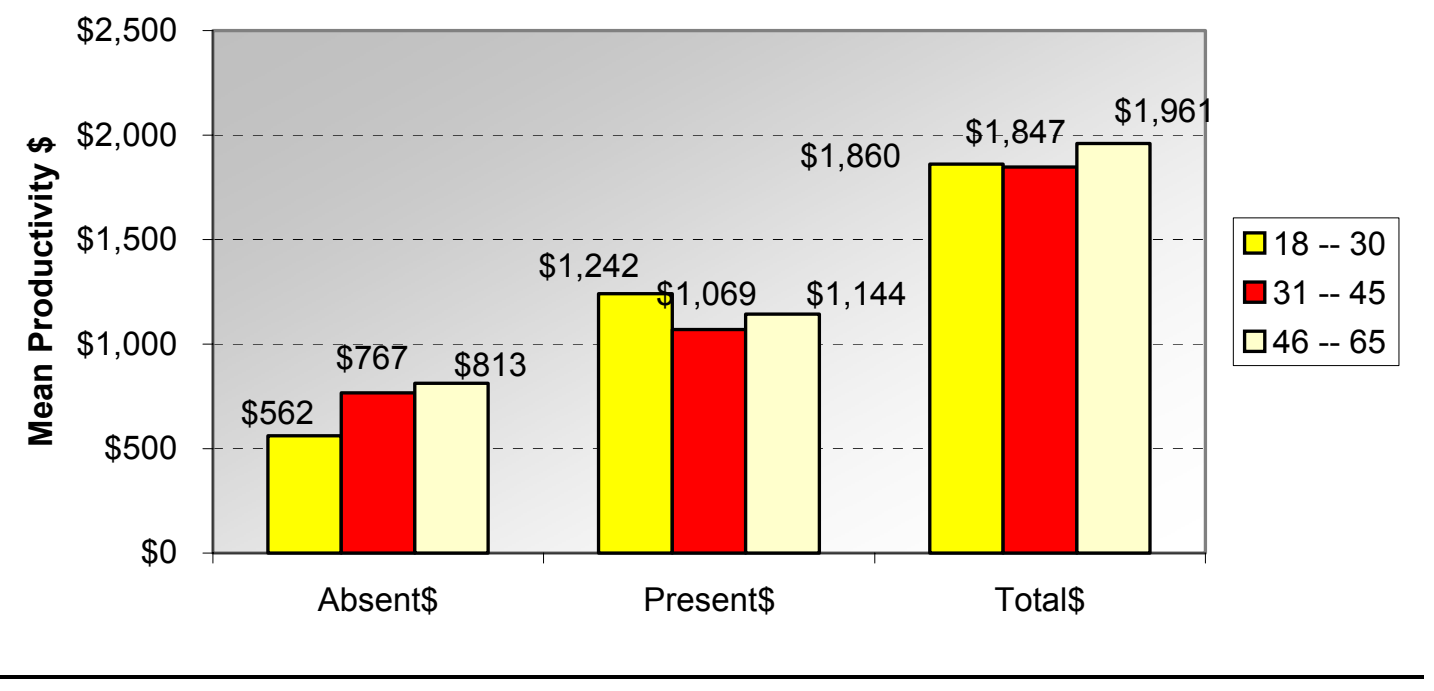


The measurement of HRQL captured the true impact of asthma and COPD in WV PEIA members from the patient perspective. Measurement of workplace productivity losses would provide the employer (state of WV) valuable information regarding the impact of asthma and COPD in the workplace. Measurement of these outcomes in Phase II has brought this study closer towards capturing a much broader impact of chronic respiratory illnesses such as asthma and COPD on patients' health. 


\section{CHAPTER FIVE}

\section{SUMMARY AND CONCLUSIONS}

This chapter presents a review of the study findings, draws conclusions, presents research implications, lists limitations for each phase of the study, and provides recommendations for future research.

\section{Phase I}

Asthma and COPD are chronic respiratory conditions impacting both morbidity and mortality. Medications for asthma and COPD are grouped into two main categories: long-acting maintenance medications, and short-acting relief medications. For the management of asthma, long-acting medications such as ICS have been recommended as the most potent and cost-effective medication, in addition to add-on maintenance agents such as long-acting beta-agonists and leukotrine inhibitors, according to NHLBI guidelines. The use of maintenance medications in patients with asthma has been shown to result in fewer adverse outcomes, and reduced healthcare utilization and costs. For COPD, no specific medications are recommended by the GOLD guidelines for long-term prophylactic use, however, some studies have demonstrated the beneficial effects of ICS in COPD in terms of alleviating symptoms, improving HRQL, and reducing mortality. In patients with asthma, adherence to maintenance medications continues to be a major concern, with less than half of them being adherent to prophylactic pharmacotherapy. In WV, there have been no studies examining the impact of medication nonadherence on healthcare utilization and costs in patients with asthma and COPD. 
Thus, the aim of this phase of the study was to examine the prevalence of medication adherence in WV state employees, and to examine the impact of medication adherence on utilization of healthcare services such as hospitalizations, ED visits, and outpatient visits, as well as related-healthcare costs. Another aim of this phase was to examine the overall prevalence of asthma and COPD in WV state employees, and to examine whether medication prescribing for asthma and COPD patients was according to NHLBI and GOLD guidelines, respectively.

\section{Conclusions for Phase I}

The conclusions for Phase I are presented based on research questions proposed in the study as follows:

Research Question 1: Examine whether medication prescribing was according to guidelines in WV PEIA members

This was an exploratory question assessing whether the prescribing of maintenance medications in WV PEIA employees with asthma and COPD was according to published guidelines. More than half of all asthma-only and both group enrollees were prescribed maintenance medications. These findings suggest that prescribing in this population is slightly better than previously reported prescribing in other populations, but there is abundant room for improvement. In the COPD-only group, it is difficult to assess whether prescribing was according to guidelines. Lack of medication use could possibly be a result of an absence of specific guidelines recommending maintenance medications (unlike NHLBI guidelines in asthma), or a perception among certain patients 
that medications are not effective, or misclassification bias, thus patients were incorrectly classified as having the disease and did not require maintenance medications.

Research Question 2: Examine the overall prevalence of asthma and COPD, and examine prevalence by demographic characteristics such as age and gender

This was another exploratory question examining the overall prevalence of asthma and COPD in WV PEIA enrollees. The null hypothesis A in this research question was that there is no difference in prevalence by age group and gender. The prevalence of asthma in WV PEIA enrollees was significantly lower than national estimates, but similar to the prevalence in WV Medicaid. For COPD, the prevalence was similar to national estimates. Overall, the prevalence of asthma and COPD increased with age, and was higher among females as compared to males for all disease groups.

Research Questions 3: Examine the extent of adherence to maintenance medications in WV PEIA members with asthma and COPD

The extent of adherence based on the absolute MPR value was low for each of the three disease groups. Adherence was lowest for the COPD-only group, and was similar for the asthma-only group, and the both groups. Higher adherence in the asthma-only and the both groups was expected since NHLBI guidelines do recommend the use of maintenance medications on a regular basis. 
Research Question 4: Examine the impact of medication adherence on healthcare utilization such as the number of adverse outcomes (hospitalizations/ED visits), outpatient visits, and LOS

The null hypotheses for this research question were that there is no difference in the number of adverse outcomes (null hypothesis B), number of outpatient visits (null hypothesis C), and LOS (null hypothesis D) between asthma and COPD patients who were adherent to maintenance medications versus those who were not. Medication adherence did affect the number of adverse outcomes, but only for the COPD-only group. Increase in medication adherence was associated with an increase in the number of adverse outcomes. Increasing medication adherence was associated with an increase in the number of outpatient visits for each of the three disease groups. LOS also increased with increasing MPR. Although utilization increased with increasing adherence, confounding by disease severity was the most likely explanation. Thus, increased medication adherence as indicated by an increasing MPR was possibly a reflection of increasing disease severity, which could have led to higher prescription drug use.

Research Question 5: Examine the impact of medication adherence on total healthcare costs

The null hypothesis $\mathrm{E}$ for this research question investigated that there is no difference in total healthcare costs between asthma and COPD patients who were adherent to maintenance medications versus those who were not. For each of the three disease groups, total healthcare costs increased with increasing medication adherence, as indicated by the MPR. Thus, similar to research question 4, increasing medication refills 
was an indicator of increasing disease severity, or reliance on prescription drug therapy for disease management, which confounded the relationship between medication adherence and total healthcare costs.

Research Question 6: Examine the relative risk of adverse health outcomes such as a hospitalization or an ED visit as a consequence of medication nonadherence

The null hypothesis $\mathrm{F}$ investigated that there was no difference in the relative risk of experiencing an adverse outcome such as a hospitalization or an ED visit between those who were adherent to maintenance medications versus those who were not. The probability of experiencing an adverse outcome increased with medication adherence for the COPD-only group. In the absence of specific guidelines recommending the prophylactic use of any specific medications, pharmacotherapy use in COPD was based on the need to take a medication due to increasing disease severity. For the asthma-only and the both groups, there was no difference in the risk of adverse outcomes between patients who were adherent versus those who were not.

\section{Phase II}

This phase of the study involved exploring the relationship between medication adherence and humanistic outcomes such as HRQL, as well as studying the impact of medication adherence on losses in workplace productivity. The relationship between medication adherence and HRQL has not been examined before. Also, the relationship between medication adherence and workplace productivity has been largely unexplored. 
This phase also examined the congruence between refill-based adherence and selfreported adherence.

\section{Conclusions for Phase II}

The conclusions for Phase II are presented based on research questions proposed in the study as follows:

Research Question 7: Examine the overall HRQL among patients with asthma and $C O P D$

The SGRQ was used to measure HRQL in WV state employees with asthma and COPD. The absolute values for total as well as domain-specific (symptoms, activity, and impacts) scores for the WV PEIA sample were similar to those obtained from other populations with asthma and COPD, thereby indicating a similar perception of HRQL, and a similar impact asthma and COPD on HRQL among WV PEIA members as compared to asthma and COPD patients in other populations. HRQL worsened with increasing disease severity. Patients with both asthma as well as COPD experienced significantly worse HRQL than patients with asthma-only or COPD-only.

Research Question 8: Examine the prevalence of adherence based on self-report

This exploratory question assessed the prevalence of medication nonadherence based on the responses to the Morisky scale. Around 40\% of asthma and COPD-patients were classified as high adherent, around 15\% were classified as medium adherent, and another $40 \%$ were classified as low adherent, in the entire phase II sample. Overall, the 
proportion of adherent patients in each of the three disease groups were similar. Based on self-report, medication adherence among PEIA members with asthma and COPD is similar to the overall prevalence of medication adherence among patients with asthma in the United States. However, respondents are more likely to report themselves as adherent, therefore, adherence rates obtained from self-report could potentially be an overestimation of the true medication adherence in WV PEIA members with asthma and COPD.

Research Question 9: Examine the congruence between self-reported and refill-based adherence

The null hypothesis G stated that there is no significant correlation between selfreported adherence and adherence measured from refill claims data. The correlation between self-report and refill adherence (MPR and median gap) was not clinically significant. Statistically significant correlations were found between MPR and Morisky adherence, and median gap and Morisky adherence, but the magnitude of the correlation was too small to assume clinical significance.

Research Question 10: Examine the impact of medication adherence on HRQL

The null hypothesis $\mathrm{H}$ this research question aimed to investigate was that there were no significant differences in HRQL between asthma and COPD patients who were adherent to maintenance medications and those who were not. For the asthma-only and the both groups, medication adherence was not a significant predictor of HRQL. For the COPD-only group, HRQL worsened with increasing MPR. Self-reported health status 
was a significant predictor of HRQL for each of the three disease groups, with HRQL worsening with deteriorating health status.

Research Question 11: Examine the impact of medication adherence on losses in workplace productivity

The null hypothesis I stated that there were no significant differences in workplace productivity dollars between asthma and COPD patients who were adherent to maintenance medications and those who were not. For each of the three disease groups, medication adherence was not a significant predictor of total productivity dollars.

\section{Study Implications}

This study was undertaken to examine medication adherence with prophylactic pharmacotherapy in patients with asthma and COPD and its impact on patient outcomes in the WV PEIA population. The findings of this study should be useful to state/private health insurance programs to help them improve the prescribing of maintenance medications for prophylactic use in asthma and COPD. This study also has implications for patients, prescribers, and decision-makers. These implications are described in the following sections.

\section{Implications to Health Insurance Programs}

The results of this study have demonstrated that there is indeed inappropriate prescribing of maintenance medications such as ICS therapy in patients with asthma and COPD. As compared to other studies, a significant proportion of patients with asthma 
received prophylactic pharmacotherapy, but there is a continual need for improvement. Although findings from this study have shown that increased adherence was associated with worse outcomes, this was most likely due to confounding due to disease severity. The importance of prophylactic pharmacotherapy given the published evidence cannot be underscored. Health insurance programs can develop and implement active interventions such as one-on-one education and individual outreach visits that will result in fewer adverse outcomes, reduced healthcare utilizations and related-costs, and also improve patients' HRQL. This study has also shown the tremendous impact that asthma and COPD have on workplace productivity. On average, total productivity losses of around $\$ 2,000$ per asthma or COPD patient per year, with around 10,000 patients translates into \$20 million annually in terms of lost productivity and lost dollars for the state. Necessary steps such as improving patient and physician education about the disease and its risk factors, as well as designing interventions that increase the use of maintenance medications, must be taken in order to reduce the burden of asthma and COPD. This could possibly result in improved outcomes from the patients' perspective (such as HRQL), and also potentially save considerable dollars due to fewer losses in workdays, and improved efficiency while at work.

\section{Implications to the Patients}

The findings of this study are of utmost relevance to the patients. Both asthma and COPD are conditions that affect the quality of life, workplace productivity, and also incur high expenses to the patients due to utilization of healthcare services (for example: insurance plans where patients pay an 80:20 deductible). These diseases also exert a 
tremendous burden on the caregivers, impact their quality of life and productivity. Thus, increased awareness regarding the use of prophylactic pharmacotherapy among patients could possibly result in patients requesting their physician to prescribe these medications. This could potentially result in reduced adverse outcomes such as hospitalizations and ED visits, and improved HRQL in patients with asthma and COPD.

\section{Implications to the Prescribers}

Although this study did not demonstrate the desired impact of prophylactic pharmacotherapy on outcomes, it did demonstrate the lack of prescribing according to guidelines, and the burden of asthma and COPD in terms of healthcare utilization and costs, and losses in workplace productivity. Prescribers should realize that the adverse outcomes associated with adherence were a consequence of disease severity, which could be alleviated through the use of appropriate prophylactic pharmacotherapy. Overall, the findings from this study could potentially result in a heightened awareness of the impact of asthma and COPD on patient outcomes.

\section{Implications to the Decision-Makers}

This study does show that prophylactic medications such as ICS are inadequately prescribed. Thus, decision-makers can develop and implement strategies that will increase appropriate prescribing of prophylactic pharmacotherapy in asthma and COPD. Decision-makers need to realize that specific interventions have to be targeted towards physicians as well as patients. Today's consumer (patient) is more proactive and has a larger influence on physician decisions. Thus, appropriate resources should be allocated 
towards directing interventions that improve patients' knowledge about the disease, its risk factors and treatment. Improved education and awareness could potentially result in better self-management of asthma and COPD, consequently resulting in better medication adherence.

\section{$\underline{\text { Study Limitations }}$}

This study had two phases: phase I used secondary data, and phase II used primary data. The limitations of both phases are listed below.

\section{Limitations of Phase I}

The study used claims data, which is collected for administrative purposes and not for conducting research. Thus use of this data has inherent limitations, since the data is subject to coding errors. The biggest concern is misclassification of diagnosis, since patients are identified based on their primary diagnosis code. If patients were incorrectly classified as having asthma or COPD, their inclusion in this study sample would be incorrect, and would dilute the MPR due to a lack of prescription claims.

The absence of data on clinically determined disease severity is a major limitation. Disease severity was a major confounder in all the analyses, and the lack of clinical data did not allow for adjustment of clinically determined disease severity.

Medication adherence in Phase I was measured using proxy indices. The use of these indices have limitations, the biggest assumption being that a prescription dispensed is a prescription taken, although these indices have been used and validated previously (Farris et al, 1994 and Christensen et al, 1997). This assumption assumes special 
significance in the case of asthma and COPD medications, where incorrect inhaler technique can further impact the actual dose that is delivered. Thus, a patient having a regular refill pattern may still be getting a suboptimal dose.

Several behavioral risk factors such as smoking impact outcomes in asthma and COPD. Also, data on socioeconomic status and physician-patient interaction, which affect medication adherence, as well as outcomes resulting from adherence, were not available. Thus, the study could suffer from potential omitted variable bias.

\section{Limitations of Phase II}

A potential limitation of phase II was that only one survey mailing was done. This yielded a relatively low overall response rate (23\%), which was especially low in the COPD-only group (15\%). Thus, it is possible that the survey respondents are not representative of the entire phase I sample.

Another limitation of this study is the endogeneity that exists between medication nonadherence and HRQL. Thus, it is difficult to conclude whether poor HRQL is a consequence of adherence, or poor HRQL resulting from the disease affected medication adherence.

The measurement of self-reported adherence as well as workplace productivity in phase II is subject to bias since respondents are more likely to report higher adherence, as well as underreport the actual number of workdays missed or work-hours lost, for the fear of being reprimanded by their employer. Thus, adherence is likely to be overestimated, whereas losses in workplace productivity are likely to be underestimated in this study sample. 
Other limitations of using a self-administered mail questionnaire apply to this study as well. Although mail questionnaires possess the advantage of being relatively inexpensive to administer, provide access to a larger population, provide anonymity and elimination of interviewer bias, there are several limitations. These include measurement errors due to respondents not understanding the instructions and the items, item nonresponse, insensitivity to substitution, and recall bias (since the recall period for the survey was one year).

\section{Directions for Future Research}

The two phases in this study have given rise to several interesting questions, which will provide the impetus for future research purposes. The first study that arises from phase I is a prospective study which uses multiple measures of medication adherence, such as claims data, self-reports, patient diaries, plus the use of an electronic device that evaluates either inhaler or peak flow meter use, and its relationship to outcomes. The inability of this study to adjust for patient severity or lifestyle modifications can be adjusted for by conducting a prospective study where patients with asthma or COPD can be identified from a specific health system and followed for a specified period of time to determine outcomes. Also the measurement of outcomes such as HRQL, can be conducted at multiple points in time, such as before and after a particular intervention designed to improve medication adherence. Also, the presence of a control group which receives either no intervention or a different type of intervention to promote adherence should allow for comparison between groups and examine the true 
impact of medication adherence after adjusting for various confounders such as disease severity.

Another future study could examine differences in prescribing patterns by physician specialty. Although some studies have examined this issue and found considerable differences by specialty, more information regarding prescribing patterns by specialty will enable decision-makers to design interventions in a more cost-effective manner by allocating resources more efficiently.

This study measured losses in workdays and inefficiencies while at work due to asthma and COPD based on patient self-report. A future follow up study should examine the correlation between patients' self-report data and data on absenteeism and short-term disability from the employer. This will help validate the measurement of productivity losses from self-report. 


\section{BIBLIOGRAPHY}

Alemayehu B, Aubert RE, Feifer RA, et al. Comparative analysis of two quality-of-life instruments for patients with chronic obstructive pulmonary disease. Value in Health 2002;5(5):437-442.

Apter AJ, Reisine ST, Affleeck G, Barrows E, Zuwallack RL. Adherence with twice daily dosing of inhaled corticosteroids. Socioeconomic and health belief differences. Am J Respir Crit Care Med 1998;157(6 pt 1):1810.

Asthma Prevalence, Healthcare Use and Mortality, 2000-2001; National Center for Health Statistics, CDC. Available at: www.cdc.gov/nchs/products/pubs/hestats/asthma/asthma.htm Accessed: 02/19/04.

Bai TR. Glucocorticosteroid treatment of asthma. Can Family Physician. 1995;41:192127.

Bailey WC, Richards JM, Brooks CM, et al. Features of asthma in older adults. J Asthma 1992;29(1):21-8.

Balkrishnan R, Anderson RT, Bowton D. Self-reported health status predictors of healthcare services utilization and charges in elderly asthmatic patients. J Asthma 2000;37(5):415-423.

Balkrishnan R, Christensen DB. Inhaled corticosteroid use and associated outcomes in elderly patients with moderate to severe chronic pulmonary disease. Clin Ther 2000;22(4):452-469.

Balkrishnan R, Christensen DB. Inhaled corticosteroid use and associated outcomes in elderly patients with moderate to severe chronic pulmonary disease. Clin Ther. 2000;22:452-469.

Balkrishnan R, Norwood GJ, Anderson A. Effects of inhaled corticosteroid pharmacotherapy introduction in asthmatic Medicaid-enrolled children. Drug Benefit Trends 1998;10(10):37-40.

Balkrishnan R, Norwood GJ, Anderson A. Outcomes and costs benefits associated with the introduction of inhaled corticosteroid therapy in a Medicaid population of asthmatic patients. Clin Ther 1998;20(3):567-580.

Barbee RA, Murphy S. The natural history of asthma. J Aller Clin Immunol 1998;102:S65-S72.

Barr JT, Schumaker GE, Freeman S, et al. American translation, modification, and validation of the St. George's Respiratory Questionnaire. Clin Ther 2000;22(9):11211145 . 
Barr R, Somers SC, Speizer FE, Camargo CA. Patient factors and medication guideline adherence among older women with asthma. Arch Intern Med 2002;162:1761-1768.

Bauman LJ, Wright E, Leickly FE, Crain E, Kruszan-Moran D, Wade SL, Visness CM. Relationship of adherence to pediatric asthma morbidity among inner city children. Pediatrics 2002;110(1):1-7.

Bender BG. Overcoming barriers to nonadherence in asthma treatment. J Allergy Clin Immunol 2002;109:S554-9.

Bergner M, Bobbit RA, Carter WB, Gilson BS. The sickness impact profile: development and final revision of a health status measure. Med Care 1981;19:787-805.

Berzon RA, Mauskoff J, Simeon GP: Choosing a health profile (descriptive) and/or a patient-preference (utility) measure for a clinical trial. In Quality of Life and Pharmacoeconomics in Clinical Trials, 2nd ed. (Spilker B, editor). Lippincott-Raven Publishers, Philadelphia, 1995.

Best Practice Medicine - Professional Reference: Asthma. Available at: http://merck.praxis.md/bpm/bpm.asp?page=BPM01AL03\&section=report. Accessed: July 13, 2002.

Blais L, Ernst P, Boivin JF, Suissa S. Inhaled corticosteroids and the prevention of readmission to the hospital for asthma. Am J Respir Crit Care Med 1998;158:126-132.

Blais L, Suissa S, Boivin JF, Ernst P. First treatment with inhaled corticosteroids and the prevention of admissions to hospital for asthma. Thorax 1998;53:1025-9.

Blanc PD, Trupin L, Eisner M, et al. The work impact of asthma and rhinitis: findings from a population-based survey. J Clin Epidemiol 2001;54(6):610-8.

Bosley CM, Fosbury JA, Cochrane GM. The psychological factors associated with poor compliance with treatment in asthma. Eur Resp J 1995;8(6):899-904.

Bousquet J, Knani J, Dhivert H, Richard A, Chicoye A, Ware JE, Francois BM. Quality of life in asthma. I. Internal consistency and validity of the SF-36 questionnaire. Am J Resp Crit Care Med 1994;149(2 part 1):371-375.

Bradley C. Compliance with drug therapy. Pres J 1999;39(1):4551.

Braunstein GL, Trinquet G, Harper AE. Compliance with nedocromil sodium and a nedocromil sodium / salbutamol combination. Compliance working group. Eur Respir J 1996;9(5):893. 
Breslin E, Schans CV, Breulink S, et al. Perception of fatigue and quality of life in patients with COPD. Chest 1998;114:958-64.

Brooks CM, Richards JM, Kohler CL, et al. Assessing adherence to asthma medication and inhaler regimens: a psychometric analysis of adult self-report scales. Med Care 1994;32(3):298-307.

Burton WN, Conti DJ, Chen CU, et al. The role of health risk factors and disease on worker productivity. J Occup Environ Med 1999;41(10):863-77.

Bush RK. The role of allergens in asthma. Chest 1992;101(6):378S-380S.

Busto SC, Gomara RE, Garcia AR, et al. Multicenter study of children's compliance to antibiotic treatment in primary care. Aten Primaria 2001;27(8):554-8.

Charlson ME, Pompei P, Ales KL, Mackenzie R. A new method of classifying prognostic comorbidity in longitudinal studies: development and validation. J Chronic Dis 1987; $40: 373-83$.

Cisternas MG, Blanc PD, Yen IH, et al. A comprehensive study of direct and indirect costs of adult asthma. J Allergy Clin Immunol 2003;111(6):1212-6.

Choo PW, Rand CS, Inui TS, et al. Validation of patient reports, automated pharmacy records, and pill counts with electronic monitoring of adherence to antihypertensive therapy. Med Care;37(9):846-857.

Christensen DB, Williams B, Goldberg HI, et al. Assessing compliance to antihypertensive medications using computer-based pharmacy records. Med Care 1997;35(11):1164-1170.

Creer TL, Wigal JK, Kotses H, et al. A life activities questionnaire for adult asthma. J Asthma 1992;29:393-9.

D'Uzro AD. Differentiating asthma from COPD in primary care. Ontario Thoracic Reviews 2001;13(1):4-13.

Data Fact Sheet: Asthma Statistics; U.S. Department of Health and Human Services, Public Health Services, National Institutes of Health, National Heart, Lung and Blood Institute, January 1999.

Dimatteo AR, Lepper HS, Croghan TW. Depression is a risk factor for noncompliance with medical treatment: meta-analysis of the effects of anxiety and depression on patient adherence. Arch Intern Med 2000;160:2101-7. 
Domingo A, Lamarca R, Ferrer M, et al. Health-related quality of life and mortality in male patients with chronic obstructive pulmonary disease. Am J Resp Crit Care Med 2002; 166:680-685.

Donahue JG. Weiss ST, Livingston JM. Goetsch MA, Greineder DK, Platt R. Inhaled corticosteroids and the risk of hospitalization for asthma. JAMA 1997;277:887-891.

Druss BG, Marcus SC, Olfson M, et al. Comparing the national economic burden of five chronic conditions. Health Aff 2001;20(6):233-41.

Endicott J, Neel J. Assessment measures for clinical studies: Endicott Work productivity Scale (EWPS): a new measure to assess treatment effects. Psychopharmacol Bull 1997;33:13-6.

Eisner MD, Ackerson LM, Chi F, Kalkbrenner A, Buchnor D, Mendoza G, Lieu T. Health-related quality of life and future healthcare utilization for asthma. Ann Allergy Asthma Immunol 2002;89:46-55.

Eraker SA, Kirscht JP, Becker MH. Understanding and improving patient compliance. Ann Intern Med 1984;100:258-68.

Erickson SR, Coombs JH, Kirking DM, Azimi AR. Compliance from self-reported versus pharmacy claims data with metered-dose inhalers. Ann Pharmacother 2001;35:997-1003.

Ernst P, Spitzer WO, Suissa S, et al. Risk of fatal and near fatal asthma in relation to inhaled corticosteroid use. JAMA 1992;268:3462-4.

Evans L, Spelman M. The problem of non-compliance with drug therapy. Drugs 1983; 25:63-76.

Evans RI, Mullally D, Wilson R, et al. National trends in the morbidity and mortality of asthma in the US: prevalence, hospitalization, and death from asthma over two decades: 1965-1984. Chest 1987;91:65S-74S.

Fairman K, Motheral B. Evaluating medication adherence: which measure is right for your program? J Manag Care Pharm 2000;6(6):499-506.

Farris KB, Kaplan B, Kirking DM. Examination of days supply in computerized prescription claims. J Pharmacoepidemiol 1994;3(1):63-76.

Feeny D, Furlong W, Barr RD, Torrance GW, Rosenbaum P, Weitzman S. A comprehensive multi-attribute system for classifying the health status of survivors of childhood cancer. J Clin Oncol 1992;10:923-928. 
Ferrer M, Villasante C, Alonso J, et al. Interpretation of quality of life scores from the St. George's Respiratory Questionnaire. Eur Resp J 2002;19(3):405-13.

Fincham JE. Medication compliance and the elderly. Journal of Pharmacoepidemiology 1995;4(2):7-14.

Fish L, Lung CL. Adherence to asthma therapy. Ann Allergy Asthma Immunol 2001;86(Suppl):24-30.

Fletcher SW, Pappius EM, Harper SJ. Measurement of medication compliance in a clinical setting: comparison of three methods in patients prescribed digoxin. Arch Intern Med 1979;139:635-8.

Gao X, Nau D. Congruence of three self-report measures of medication adherence among HIV patients. Ann Pharmacother 2000;34(10):1117-22.

Gergen PJ, Weiss KB. Changing patterns of asthma hospitalization among children. JAMA 1990;264:1688-1692.

Global Initiative for Chronic Obstructive Lung Disease. Global Strategy for the Diagnosis, Management, and Prevention of Chronic Obstructive Lung Disease: NHLBI/WHO Workshop Report.

Goetsel RZ, Ozminkowski RJ, Long S. Development and reliability analysis of the Work Productivity Short Inventory (WPSI) instrument measuring employee health and productivity. J Occup Environ Med 2003;45:743-762.

Grasso ME, Weller WE, Shaffer TJ, et al. Capitation, managed care, and chronic obstructive pulmonary disease. Am J Respir Crit Care Med 1998;158:133-8.

Greenberg RN. Overview of patient compliance with medication dosing: a literature review. Clin Ther 1984;6:592-99.

Grymonpre RE, Didur CD, Montgomery PR, Sitar DS. Pill count, self-report, and pharmacy claims data to measure medication adherence in the elderly. Ann Pharmacother 1998;32:749-54.

Guyatt GH, Walter S, Norman G. Measuring change over time: assessing the usefulness of evaluative instruments. J Chron Dis 1987;40:171-8.

Guyatt GH, Kirshner B, Jaeschke R. Measuring health status: what are the necessary measurement properties? J Clin Epidemiol 1992;45:1341-5.

Guyatt GH, Juniper EF, Walter SD, et al. Interpreting treatment effects in randomized trials. BMJ 1998;101:163-70. 
Hajiro T, Nishimura K, Tsukino M, et al. A comparison of the level of dyspnea vs disease severity in indicating the health-related quality of life in patients with COPD. Chest 1999;116:1632-1637.

Halpern MT, Stanford RH, Borker R. The burden of COPD in the USA: results from the confronting COPD survey. Respir Med 2003;97 (Suppl C):S81-S89.

Hatoum HT, Schumock GT, Kendzierski DL. Meta-analysis of controlled trials of drug therapy in mild chronic asthma: the role of inhaled corticosteroids. Ann Pharmacother. 1994;28:1285-89.

Haynes RB. Introduction. In: Haynes RB, Taylor DW, Dackett DL, eds. Compliance in Healthcare. Baltimore, Md: The Johns Hopkins University Press; 1979:1-7.

Hilleman DE, Dewan N, Maleker M, et al. Pharmacoeconomic evaluation of COPD. Chest 2000;118:1278-85.

Huang YC, Morisky DE. Stability of blood pressure: is a sequential blood pressure reading protocol efficient for a large-scale community screening program? J Hum Hypertens 1999;13(9):637-42.

Hunt SM, McKenna SP, McEwan J, et al. A quantitative approach to perceived health status: a validation study. J Epidemiol Community Health 1980;34:281-286.

Hurd S. The impact of COPD on Lung Health Worldwide: Epidemiology and Incidence. Chest 2000;117:1S-14S.

Huse DM, Russell MW, Weiss ST, Hartz SC. Anti-inflammatory therapy reduces total costs of asthma care compared with bronchodilation: the asthma outcomes registry. Am J Manag Care 2000;6:1045-50.

Hyland ME, Ley A, Fisher DW, Woodward V. Measurement of psychological distress in asthma and asthma management programmes. Br J Clin Psychol 1995;34(4):601-11.

Hyland ME. The living with asthma questionnaire. Respir Med 1991;85:13-6.

Jacobson L, Hertzman P, Lofdahl CG, et al. The economic impact of asthma and chronic obstructive pulmonary disease (COPD) in Sweden in 1980 and 1981. Resp Med 2000;94:247-255.

James PN, Anderson JB, Prior JG, et al. Patterns of drug taking in patients with chronic airflow obstruction. Postgrad Med J 1985;61:7-10.

Janssens JP. When and how to assess quality of life in chronic lung disease. Swiss Med Wkly 2001;131:623-629. 
Jeffrey PK. Differences and similarities between chronic obstructive pulmonary disease and asthma. Clin and Exper Allergy 1999;29(2):14-26.

Jones PW, Baveystock CM, Littlejohns P. Relationships between general health measured with the Sickness Impact Profile and respiratory symptoms, physiological measures, and mood in patients with chronic airflow limitation. Am Rev Resp Dis 1989;140:1538-43.

Jones PW, Quirk FH, Baveystock CM. The St. George's Respiratory Questionnaire. Resp Med 1991;85(Suppl):25-31.

Jones PW, Quirk FH, Baveystock CM, et al. A self-complete measure of health status for chronic airflow limitation: the St. George's Respiratory Questionnaire. Am Rev Resp Dis 1992;145:1321-7.

Jones PW. Issues concerning quality of life in COPD. Chest 1995;107(5):187S-193S.

Jones PW. Nedocromil Sodium quality of life study group. Quality of life, symptoms, and pulmonary function in asthma: long-term treatment with nedocromil sodium examined in a controlled, multicenter trial. Eur Respir J 1994;7:55-62.

Joshi AV, Smith MJ. Rates of asthma prevalence and healthcare services utilization in a Medicaid population by demographic characteristics. Poster Presentation at the $19^{\text {th }}$ International Conference on Pharmacoepidemiology and the $1^{\text {st }}$ International Conference on Risk Management, August 21-24, Philadelphia, PA. Abstract published Pharmacoepidemiology and Drug Safety 2003;12:S39.

Juniper EF, Guyatt GH, Willan A, Griffith LE. Determining a minimal important change in disease-specific quality of life questionnaire. J Clin Epidemiol 1994;47(1):81-87.

Juniper EF. Quality of life considerations in the treatment of asthma. Pharmacoeconomics 1995;8(2):123-138.

Juniper EF. Quality of life questionnaires: does statistically significant = clinically important? [editorial]. J Allergy Clin Immunol 1998;102:16-17.

Juniper EF. Health-related quality of life in asthma. Curr Opin Pulm Med 1999;5:105110.

Juniper EF, Guyatt GH, Epstein RS, et al. Evaluation of impairment of health-related quality-of-life in asthma: development of a questionnaire for use in clinical trials. Thorax 1992;47:76-83.

Juniper EF, Guyatt GH, Ferrie PJ, et al. Measuring quality of life in asthma. Am Rev Respir Dis 1993;147:832-8. 
Juniper EF, Johnston PR, Borkhoff CM, Guyatt GH, Boulet LP, Haukioja A. Quality of life in asthma clinical trials: a comparison of salmeterol and salbutamol. Am J Resp Crit Care Med 1995;151:66-70.

Kalton G (Ed.) (1987), Introduction to survey sampling, Sage University Paper Series on Quantitative Applications in Social Sciences, Series/Number 07-035. Beverly Hills and London: Sage Pubns.

Kamp DW. Physiologic evaluation of asthma. Chest 1992;101(6):396S-400S.

Kaplan RM, Anderson JP, Wu AW, Matthews WC, Cozin F, Orenstein D. The Quality of Well-Being scale: applications in AIDS, cystic fibrosis, and arthritis. Med Care 1989;27(suppl):S27-43.

Kelloway JS, Wyatt RA, Adlis SA. Comparison of patients' compliance with prescribed oral and inhaled asthma medications. Arch Intern Med. 1994;154:1349-1352.

Kelloway JS, Wyatt RA, Adlis SA. Comparison of patients' compliance with prescribed oral and inhaled medications. Arch Intern Med 1994;154:1349-52.

Kessler RC, Greenburg PE, Mickelson KD, et al. The effects of chronic medical conditions on work loss and work cutback. J Occup Environ Med 2001;43:218-225.

Kessler RC, Ormel J, Demler O, Stang PE. Comorbid mental disorders account for the role impairment of commonly occurring chronic physical disorders: results from the national comorbidity survey. J Occup Environ Med 2003;45(12):323-331.

Lahdensuo A, Haahtela T, Herrala J, et al. Randomised comparison of guided selfmanagement and traditional treatment of asthma over one year. BMJ 1996; 312:748-52.

Landry JA, Smyer MA, Tubman JG, et al. Validation of two methods of data collection of self-reported medicine use among the elderly. Gerontologist 1988; 28:672-6.

Larson JL, Kapella MC, Wirtz S, Covey MK, Berry B. Reliability and validity of the Functional Performance Inventory in patients moderate to severe chronic obstructive pulmonary disease. J Nurs Measurement 1998;6(1):55-73.

Le Coq EM, Colland VT, Boeke AJ, Bezemer DP, Van Eijk JT. Reproducibility, construct validity, and responsiveness of the "How Are You" (HAY), a self-report quality of life questionnaire for children with asthma. J Asthma 2000;37(1):43-58.

Lerner D, Amick BC, Rogers WH, et al. The work limitations questionnaire. Med Care 2001;39(1):72-85. 
Leickly FE, Wade SL, Crain E, et al. Self-reported adherence, management behavior, and barriers to care after an emergency department visit by inner city children with asthma. Pediatrics 1998;101(5):1-8.

Leidy NK, Coughlin C. Psychometric performance of the asthma quality of life questionnaire in a US sample. Qual Life Res 1997;7:127-134.

Leidy NK. Functional status towards the progress of merry-go-rounds: toward a coherent analytical framework. Nurs Res 1994;43(4):196-202.

Leidy NK. Functional performance in people with chronic obstructive pulmonary disease. Image 1995;27(1):23-34.

Leidy NK, Haase JE. Functional performance in people with chronic obstructive pulmonary disease: a quantitative analysis. Adv Nurs Sci 1996;18(3):77-89.

Leidy NK. Psychometric performance of the Functional Performance Inventory in patients with chronic obstructive pulmonary disease. Nurs Res 1999;48(1):20-28.

Lemanske RF. Mechanisms of airway inflammation. Chest 1992;101(6):372S-377S.

Lydick E, Epstein RS, Interpretation of quality of life changes. Qual Life Res 1993;2:221-6.

Mahler DA. How should health-related quality of life be assessed in patients with COPD? Chest 2000;117:54S-57S.

Mahler DA, Mackowiak JI. Evaluation of the short-form 36-item questionnaire to measure health-related quality of life in patients with COPD. Chest 1995, 107:1585-89.

Maille AR, Kaptein AA, Koning CJ, Zwinderman AH. Developing a quality of life questionnaire for patients with respiratory illness. Monaldi Arch Chest Dis 1994;49(1):76-8.

Malone DC, Lawson KA, Smith DH. Asthma: an analysis of high-cost patients. Pharm Pract Manage Q 2000;20;12-20.

Mannino D, Homa D, Pertowski C, Ashizawa A, Nixon L, Johnson C. Surveillance for asthma-United States, 1960-1995. MMWR 1998;47(ss1):1-28.

Mapel DW, Hurley JS, Frost FJ, et al. Healthcare utilization in chronic obstructive pulmonary disease: a case-control study in a health maintenance organization. Arch Intern Med 2000;160:2653-8.

Marks GB, Dunn SM, Woolcock AJ. A scale for the measurement of quality of life in adults with asthma. J Clin Epidemiol 1992;45:461-72. 
Marks GB, Dunn SM, Woolcock AJ. An evaluation of an asthma quality of life questionnaire as a measure of change in adults with asthma. J Clin Epidemiol 1993;46:1103-11.

Morisky DE, Green LW, Levine DW. Concurrent and predictive validity of a selfreported measure of adherence. Medical Care 1986;24(1):67-74.

Murray CJL, Lopez AD. Evidence-based health policy: lessons from the global burden of disease study. Science 1996;274:740-743.

National Heart, Lung and Blood Institute, National Asthma Education and Prevention Program: Expert Panel Report 2 -Guidelines for the Diagnosis and Management of Asthma. National Institutes of Health; July 1997: Publication No. 97-4051.

National Institutes of Health, National Heart, Lung, and Blood Institute. Chronic Obstructive Pulmonary Disease. NIH Pub. No. 95-2020. Washington, D.C.: U.S. Government Printing Office, 1995.

National Pharmaceutical Council: Emerging issues in pharmaceutical cost containment. Reston, VA: 2(2): 1-6 (1992).

Nelson E, Wasson J, Kirk J, Keller A, Clark D, Dietrich A, et al. Assessment of function in routine clinical practice:description of the COOP chart method and preliminary findings. J Chronic Dis 1987;40(supp 1):S55.

Nestor A, Calhoun AC, Dickson M, Kalik CA. Cross-sectional analysis of the relationship between national guideline recommended asthma drug therapy and emergency/hospital use within a managed care population. Ann Allergy Asthma Immunol 1998;81:327-330.

NHLBI morbidity and mortality chartbook, 1998. Available at www.nhlbi.nih.gov/resources/docs/cht-book.htm. Accessed: August 29, 2002.

Nocon A, Booth T: The social impact of asthma. Fam Pract 1991:8(1):37-41.

Northfield M, Patel RK, Richardson A, et al. Lifestyle changes in mild asthma during intermittent symptom-related use of terbutaline inhaled via Turbohaler. Curr Med Res Opin 1991;12:441-449.

O’Hanrahan M, O’Malley K. Compliance with drug treatment. Br Med J. 1981;283:298-300.

Oettgen HC, Geha RS. Molecular mechanisms in allergy and clinical immunology. J Allergy Clin Immunology 2001;107:429-40. 
Olivieri D, Chetta A, Del Donno M, Bertorelli G, Casalini A, et al. Effect of short-term treatment with low-dose inhaled fluticasone propionate on airway inflammation and remodeling in mild asthma: a placebo controlled study. Am J Resp Crit Care Med 1997;155(6):1864-1871.

Osman L. The patient perspective. What should a new anti-asthma agent provide? Drugs 1996;52(Suppl):29-35.

Pauwels RA, Buist AS, Ma P, et al. Global strategy for the diagnosis, management, and prevention of chronic obstructive pulmonary disease: National Heart, Lung, and Blood Institute and World Health Organization Global Initiative for Chronic Obstructive Lung Disease (GOLD): executive summary. Respir Care 2001;46:798-825.

Peters DH, Faulds D. Salmeterol: an appraisal of its quality-of-life benefits and potential pharmacoeconomic positioning in asthma. Pharmacoeconomics 1995;7(6):562-574.

Peterson GM, Mclean S, Millingen KS. Determinants of patient compliance with antihypertensive therapy. Epilepsia 1982;23:607-613.

Pratt RJ, Robinson N, Loveday HP, et al. Adherence to antiretroviral therapy: appropriate use of reporting in clinical practice. HIV Clin Trials 2001;2(2):146-159.

Puchelle E, Vargaftig BB. Chronic obstructive pulmonary disease: an old disease with novel concepts and drug strategies. Trends in Pharmacological Sciences 2001;22(10):495-7.

Rand CS, Nides M, Cowles MK, et al. Long-term metered dose inhaler adherence in a clinical trial. Am J Resp Crit Care Med 1995;152:580-588.

Rand CS, Wise RA, Nides M, et al. Metered-dose inhaler adherence in a clinical trial. Am Rev Respir Dis 1992;146(6):1559-64.

Rennard SI. COPD: Overview of definitions, epidemiology, and factors influencing its development. Chest 1998;113:235S-241S.

Reilly MC, Tanner A, Meltzer EO. Work, classroom, and activity impairment instruments: validation studies in allergic rhinitis. Clin Drug Invest 1996;11:278-288.

Ringdal N, Whitney JG, Summerton L. Problems with inhaler technique and patient preference for oral therapy - tablet zafirlukast vs inhaled beclomethasone. Am J Respir Crit Care Med 1998;157:A416.

Rowe BH, Keller JL, Oxman AD. Effectiveness of steroid therapy in acute exacerbations with asthma: a meta analysis. Am J Emer Med. 1992;10:301-310. 
Rowe BH, Oxman AD. Performance of an asthma quality of life questionnaire in an outpatient setting. Am Rev Respir Dis 1993;148:675-81.

Ruchlin HS, Dasbach EJ. An economic overview of chronic obstructive pulmonary disease. Pharmacoeconomics 2001;19(6):623-642.

Rudd P. Helping patients follow the treatments you prescribed. In: Sackett DL, Haynes RB, Guyatt GH, Tugwell P, eds. Clinical Epidemiology. A basic science for clinical medicine. $2^{\text {nd }}$ ed. Toronto: Little, Brown and Company, 1991: 249-61.

Rutten van Molken M, Doorslaer EK, Jansen MC, Kertjens HA, Rutten FF. Costs and effects of inhaled corticosteroids and bronchodilators in asthma and chronic obstructive pulmonary disease. Am J Resp Crit Care Med 1995;151:1975-82.

Rutten van Molken MPH, Custers F, van Doorslaer EKA, Jansen MCM, Heurman L, Maesen EPV, Smeets SJ, Bommer AM, Raajimakers J. Comparing the performance of four different instruments in evaluating the effects of salmeterol in asthma quality of life. Eur Resp J 1995;5:888-898.

Sackett DL, Chambers LW, MacPherson AS, et al. The development and application of indices of health: general methods and summary of results. Am J Public Health 1977;67:423-8.

Schipper H, Clinch J, Powell V: Definitions and conceptual issues. In Quality of Life and Pharmacoeconomics in Clinical Trials. Edited by Spilker B. Philadelphia: LippincottRaven Publishers; 1996:11-23.

Schmeir JK, Leidy NK. The complexity of treatment adherence in adults with asthma: challenges and opportunities. J Asthma 1998;35(6):455-72.

Segal R, Reid LD, Mackowiak J. Cost of asthma illnesses: emergency department visits without admission. Pharm Prac Manage Q 1995;15:72-82.

Selroos O, Lofroos AB, Pietinalho A, Riska H. Asthma control and steroid doses 5 years after early or delayed introduction of inhaled corticosteroids in asthma; a real-life study. Respir Med 2004;98(3):254-62.

Selroos O, Pietinalho A, Lofroos A, Riska H. Effect of early vs late intervention with inhaled corticosteroids in asthma. Chest 1995;108:1228-34.

Selroos O, Pietinalho A, Lofroos AB, Riska H. Effect of early versus late intervention with inhaled corticosteroids in asthma. Chest 1995;108(5):1228-1234.

Sen SS, Gupchup GV, Thomas JT3. Selecting among health-related quality of life instruments. Am J Health-Syst Pharm 1999;56:1965-73. 
Spencer S, Calverley PM, Burge PS, et al. Health status deterioration in patients with chronic obstructive pulmonary disease. Am J Resp Crit Care Med. 2001;163:122-128.

Sin DD, Stafinski T, Ng YC, et al. The impact of chronic obstructive pulmonary disease on work loss in the United States. Am J Respir Crit Care Med 2002;165:704-707.

Smith MJ, Rascati KL, Johnsrud MT. Costs and utilization patterns associated with persistent asthma: a comparison of Texas Medicaid patients with and without continuous inhaled corticosteroid treatment. J Managed Care Pharm 2001;7(6):452-459.

Stanford R, McLaughlin T, Okamoto LJ. The cost of asthma in the emergency department and the hospital. Am J Respir Crit Care Med 1999;160:211-5.

Steiner JF, Fihn SD, Blair B, Inui TS. Appropriate reductions in compliance among wellcontrolled hypertensive medications. J Clin Epidemiol 1991;44:1361-1371.

Steiner JF, Koepsel TD, Fihn SD, et al. A general method of compliance assessment using centralized pharmacy records: description and validation. Med Care 1988;26(8):814-23.

Steiner JF, Prochazka AV. The assessment of refill compliance using pharmacy claims records: methods, validity and applications. J Clin Epidemiol 1997;50(1):105-116.

Stempel DA, Carlson AM, Buchner DA. Asthma: benchmarking for quality improvement. Ann Allergy Asthma Immunol 1997;79:517-24.

Stewart AL, Hays R, Ware JE. The MOS short-form general health survey: reliability and validity in a patient population. Med Care 1988;26:724-32.

Stewart M. The validity of an interview to assess a patient's drug-taking. Am J Prev Med 1987;3:95-100.

Stewart WF, Ricci JA, Chee Elsbeth, Morganstein D. Lost productive work time costs from health conditions in the United States: results from the American Productivity Audit. J Occup Environ Med 2003;45:1234-46.

Strassels SA, Smith D, Sullivan S, Mahajan PS. The costs of treating COPD in the United States. Chest 2001;119:344-352.

Suissa S, Blais L, Ernst P. Patterns of increasing beta-agonist use and the risk of fatal or near-fatal asthma. Eur Resp J 1994;7:1602-9.

Suissa S, Ernst P, Benayoun S, Baltzan M, Cai B. Low-dose inhaled corticosteroids and the prevention of death from asthma. N Engl J Med. 2000;343:332-6. 
Suissa S, Ernst P, Kezouh H. Regular use of inhaled cortisteroids and the long-term prevention of hospitalization for asthma. Thorax 2002;57(10):880-4.

Sullivan SD, Ramsey SD, Lee TA. The economic burden of COPD. Chest 2000;117:5S9S.

Tashkin DP. Multiple dose regimens. Impact on compliance. Chest 1995;107(Suppl):176S-182S.

The EuroQol Group: A new facility for the measurement of health related quality of life. Health Policy 1990;16:199-208.

Torrance GW. Measurement of health state utilities for economic appraisal. J Health Econ 1986;5:1-30.

Ungar WJ, Coyte PC. Measuring productivity loss in asthma patients: the pharmacy medication advisory system and the advisory board. Health Economics 2000;9(1):37-46.

US Department of Labor: Bureau of Labor Statistics. 2002 State Occupational Employment and Wage Estimates: West Virginia, 2003.

Van der Molen T, Postma DS, Schreurs AJ, Bowveld H, Sears MR, Meyboom de Jong B. Discriminative aspects of two generic and two asthma specific instruments: relation with symptoms, bronchodilator use, and lung function in patients with mild asthma. Qual Life Res 1997;6:353-361.

Van Schyak CP, vanGrunsven PM, Dekhuijzen PNR. Do patients from COPD benefit from treatment with inhaled corticosteroids? Eur Respir J. 1996;9:1969-1972.

Vollmer WM, Markson LE, O'Connor E, Sanocki LL, Fitterman L, Berger M, Buist AS. Association of asthma control with healthcare utilization and quality of life. Am J Respir Crit Care Med 1999;160:1647-1652.

Wandless I, Mucko JC, Smith A, Prudham D. Compliance with prescribed medicines: a study of the elderly patients in the community. J Royal Coll Gen Prac 1979;29:391-6.

Wang P. A bivariate zero-inflated negative binomial regression model for count data with excess zeroes. Economic Letters 2003;78(3):373-8.

Wang PS, Beck A, Berglund P, et al. Chronic medical conditions and work performance in the health and work performance questionnaire calibration surveys. J Occup and Environ Med 2003;45(12):1303-11.

Ward HJ, Morisky DE, Lees NB, Fong R. A clinic and community-based approach to hypertension control for an underserved minority population: design and methods. Am J Hypertens 2000;13(2):177-83. 
Ward MM, Javitz HS, Smith WM, et al. Direct medical cost of chronic obstructive pulmonary disease in the USA. Respir Med 2000;94:1123-9.

Watts RW, Mclenan G, Bassham I, et-Saadi O. Do patients with asthma fill their prescription? A primary study. Aus Fam Physician 1997;26(Suppl 1):s4.

Weiss K, Gergen P, Hodgson T. An economic evaluation of asthma in the United States. N Engl J Med. 1992;326:862-868.

Weiss K, Sullivan SD. The health economics of asthma and rhinitis. I. Assessing the economic impact. J Allergy Clin Immunol 2001;107:3-8.

Wilson L, Devine EB, So K. Direct medical costs of chronic obstructive pulmonary disease: chronic bronchitis and emphysema. Respir Med 2000;94:204-13.

Wilson CB, Jones PW, O'Leary CJ, et al. Validation of the St. George's Respiratory Questionnaire in bronchiectasis. Am J Resp Crit Care Med 1997;156:536-41.

Wijnhoven HA, Kriegsman DM, AE Hesselink, Penninx BW, de Haan M. Determinants of different dimensions of disease severity in asthma and COPD: pulmonary function and health-related quality of life. Chest 2001;119:1034-1042.

Wu AW, Young Y, Skinner EA, et al. Quality of care and outcomes of adults with asthma treated by specialists and generalists in managed care. Arch Intern Med 2001;161:2554-60. 
APPENDICES 


\section{APPENDIX A: CLAIM FILES}

Demographic File

1. RECIPIENT NUMBER (DE-IDENTIFIED)

2. PATIENT LAST NAME

3. PATIENT FIRST NAME

4. PATIENT ADDRESS

5. PATIENT BIRTHDATE

6. PATIENT GENDER

7. ELIGIBILITY BEGIN DATE

8. ELIGIBILITY END DATE

Medical Claims File

1. RECIPIENT NUMBER (DE-IDENTIFIED)

2. FIRST DATE OF SERVICE

3. LAST DATE OF SERVICE

4. PROCEDURE CODES

5. DIAGNOSIS CODES

6. DIAGNOSIS CODE DESCRIPTION

7. BILLED AMOUNT

8. NOT COVERED AMOUNT

9. PAID AMOUNT

10. DEDUCTIBLE

11. DRG CODE

12. INPATIENT/OUTPATIENT CODE 


\section{Pharmacy Claims File}

1. RECIPIENT NUMBER (DE-IDENTIFIED)

2. DATE PRESCRIPTION FILLED

3. NDC CODE

4. DRUG DESCRIPTION

5. NEW/REFILL CODE

6. METRIC QUANTITY

7. DAYS SUPPLY

8. BILLED AMOUNT

9. PAID AMOUNT

10. PAID DATE 


\section{APPENDIX B: CODES FOR IDENTIFICATION}

ICD-9-CM Codes Used to Identify Medical Claims for Asthma and COPD

Asthma

Bronchitis/bronchiolitis

Unspecified Bronchitis

Chronic Bronchitis

Emphysema

Chronic Airway Obstruction 493.xx

466.xx

490.xx

491.xx

492.xx

496.xx 


\section{APPENDIX C: MEDICATIONS}

Inhaled Corticosteroids

Aerobid, Advair, Azmacort, Beclovent, Flovent, Intal, Pulmicort,

Tilade, Vanceril

Leukotrine Modifiers

Accolate, Singulair, Zyflo

Long-Acting

Foradil (Formoterol), and Serevent (Salmeterol)

Bronchodilators

Theophyline

Theo-Dur, Theolair

Bronchodilators

Oral Corticosteroids

Delta-Cortef, Deltasone, Medrol

Short-Acting

Brethaire, Bricanyl, Maxair, Tornalate, Ventolin, Proventil

Bronchodilators

(Albuterol)

Anticholinergic

Atrovent (Ipratropium)

Bronchodilator 
COVERLETTER AND SURVEY 


\section{APPENDIX D: COVERLETTER}

Dear Sir/Madam:

Respiratory illnesses such as asthma and chronic obstructive pulmonary disease (chronic bronchitis, emphysema, chronic airways obstruction) are major public health problems in West Virginia. They are among the leading causes of mortality and morbidity in West Virginia. We at the West Virginia University School of Pharmacy, with the approval of the Public Employees Insurance Agency (PEIA) are trying to understand these important concerns from the perspective of healthcare utilization and patient health-related quality of life. This study is part of a doctoral (Ph.D.) research project.

We were able to identify you as a patient who may have asthma or chronic obstructive pulmonary disease from your medical utilization insurance claims, for which we were given approval by PEIA. Please find attached a survey that measures health-related quality of life among patients with asthma and / or chronic obstructive pulmonary disease. We would really appreciate it if you will kindly take a few minutes to complete the survey and return it in the postage-paid business reply envelope.

Your participation in this survey is voluntary and will not affect your PEIA services in any way. Your responses will be coded, and your name will not appear in any reports. Your names will be stored in the master codebook by the investigator. This codebook will be destroyed upon completion of the study. Therefore, data will be kept as confidential as legally possible.

Although we hope that you answer all of the questions, you do not have to answer any question that makes you uncomfortable. However, your response will provide useful information that is very important to the results of this study. Hence, your participation in this study will be very much valued.

If you have any questions or concerns, please do not hesitate to contact us at (304) 2936991 or (304) 293-1652 at the West Virginia University School of Pharmacy. Thank you very much for your time and effort.

Sincerely,

Ashish V. Joshi, M.S.

Doctoral Candidate

cc: Tom Susman

Director

PEIA
S. Suresh Madhavan, M.B.A, Ph.D.

Professor and Chairperson 
APPENDIX E: SURVEY 


\section{RESPIRATORY QUESTIONNAIRE}

INSTRUCTIONS: This questionnaire is designed to help us researchers understand how your respiratory illness may be troubling you and how it affects your life. We are using it to find out which aspects of your illness cause you most problems. Please answer each question by checking the box that best describes your situation. If you are not sure how to answer, please give the best answer you can. Please do not spend a long

time deciding on your answers.

\section{PART 1 - Demographics}

Age: years

Gender: $\square$ Male $\square$ Female

What is the highest level of education that you have completed?
- $8^{\text {th }}$ or less than $8^{\text {th }}$ grade
$\square$ College 1 to 3 years (some college/technical school)

$\square 9^{\text {th }}$ to $12^{\text {th }}$ grade/high school graduate

$\square$ College 4 years or more (college graduate)

Before completing the rest of the questionnaire, please check one box to show how you rate your present health: $\square$ Excellent $\square$ Very Good $\square$ Good $\square$ Fair $\square$ Poor

\section{PART 2 - One Year Description}

These are questions about how often your lung/respiratory problems have affected you over the last year. Please check ONE box for each question.

$\begin{array}{ccccc}\begin{array}{c}\text { Almost } \\ \text { every }\end{array} & \begin{array}{c}\text { Several } \\ \text { days a }\end{array} & \begin{array}{c}\text { A few } \\ \text { days a }\end{array} & \begin{array}{c}\text { Only with } \\ \text { lung or } \\ \text { day }\end{array} & \begin{array}{c}\text { Not } \\ \text { week }\end{array} \\ \text { month } & \begin{array}{c}\text { respiratory } \\ \text { infections }\end{array} & \text { all }\end{array}$

1) Over the last year, I have coughed

2) Over the last year, I have brought up phlegm (sputum)

3) Over the last year, I have had shortness of breath

4) Over the last year, I have had episodes of wheezing

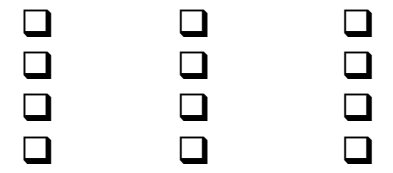

5) During the last year, how many severe or very unpleasant episodes of lung/respiratory problems have you had?
More than 3 episodes
3 episodes
2 episodes
1 episode
No episode

6) How long did the worst episode of lung/respiratory problem last? Please go to Question 7 if you didn't have a severe episode.

$\square$ A week or more $\square 3$ or more days $\square 1$ or 2 days $\square$ Less than a day

7) Over the last year, in an average week, how many good days (with few lung/respiratory problems) have you had?
$\square$ None
1 or 2
3 or 4
Nearly every day
Every day

8) If you wheeze, is it worse in the morning? If you don't wheeze, go to Part 3.

$\square$ No $\square$ Yes 


\section{PART 3}

\section{SECTION 1:}

How would you describe your lung/respiratory condition? Please check ONE box only.

The most important problem I have

Causes me a few problems

Causes me quite a lot of problems

Causes no problem

How does your respiratory condition affect your job? Please check ONE box only.

My lung/respiratory problem made me stop my job

My lung/respiratory problem interferes with my job or made me change my job

My lung/respiratory problem does not affect my job

Do not hold a job

SECTION 2: These are questions about activities that usually make you feel short of breath.

Please indicate True or False for each activity that applies to you now.

\section{True False}

Sitting or lying still

Washing yourself or dressing

Walking in the house

Walking outside on level ground.

Walking up a flight of stairs

Walking up hills....

Playing sports or active games (baseball, tennis, etc)

SECTION 3: These are more questions about your cough and shortness of breath.

Please indicate True or False for each activity that applies to you now.

$\underline{\text { True }}$ False

Coughing hurts

Coughing makes me tired

I am short of breath when I talk....

I am short of breath when I bend over.

My coughing or breathing disturbs my sleep.

I become exhausted easily...

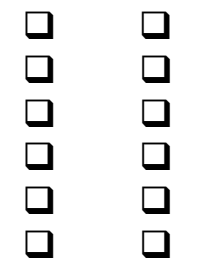

SECTION 4: These are questions about other effects that your lung/respiratory problems may

have on you. Please indicate True or False for each activity that applies to you now.

True $\underline{\text { False }}$

My cough or breathing is embarrassing in public

My lung/respiratory problem is a nuisance to my family, friends or neighbors.........

I panic or get afraid when I cannot catch my breath.

I feel that I am not in control of my lung/respiratory problem.

I do not expect my lung/respiratory problem to get any better....

I have become frail or an invalid because of my lung/respiratory problem

Exercise is not safe for me...

Everything seems too much of an effort 
SECTION 5: These are questions about your lung/respiratory medications, including oxygen, inhalers, and pills. If you are not receiving medications go to SECTION 6.

Please indicate True or False for each activity that applies to you now.

My lung/respiratory medication does not help me very much.......................
I get embarrassed using my lung/respiratory medication in public...................
I have unpleasant side effects from my lung/respiratory medication..................
My lung/respiratory medication interferes with my life a lot........................

SECTION 6: These are questions about how your activities might be affected by your breathing problem. For each question, answer True if one or more parts apply to you because of your breathing problem. Otherwise answer False.

True $\underline{\text { False }}$

I take a long time to get washed or dressed...................................... $\quad \frac{T}{\square}$

I cannot take a bath or shower, or I take a long time to do it.................... $\square \square$

I walk slower than other people my age, or I stop to rest........................ $\square$

Jobs such as household chores take a long time, or I have to stop to rest............. $\square \square$

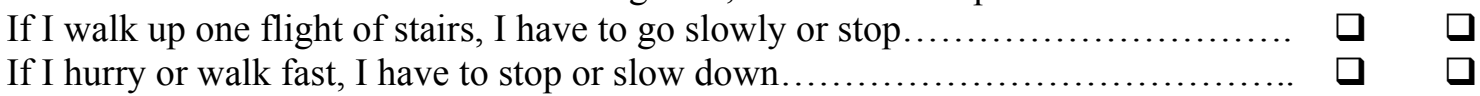

My breathing makes it difficult to do things such as walking up hills, carrying things upstairs, light gardening such as weeding, dancing, playing golf, or light sports such as horseshoes.

My breathing problem makes it difficult to do things such as carrying heavy loads, digging in the garden or shovelling snow, jogging

or walking briskly, playing tennis or swimming.

My breathing problem makes it difficult to do things such as very heavy manual labor, riding a bike, running, swimming fast, or playing competitive sports.

SECTION 7: We would like to know how your breathing usually affects your daily life.

Please indicate True or False for each activity that applies to you now.

$\underline{\text { True }} \underline{\text { False }}$

I cannot play sports or active games.

I cannot go out for entertainment or recreation

I cannot go out of the house to do the grocery shopping

I cannot do household chores.

I cannot move far from my bed or chair

Here are examples of activities that your lung/respiratory problem may prevent you from doing.

Going for walks or walking the dog

Doing activities or chores at home or in the garden

Going to church, or a place of entertainment

Going out in bad weather or into smoky rooms

Visiting family or friends or playing with children 
Please list some important activities (from those listed above or other) that your lung/respiratory problem may be currently stopping you from doing:

The following questions describe how your breathing problems affect you. Please indicate which one of the following applies to you (Please check ONE box only).

It does not stop me from doing anything I would like to do

It stops me from doing one or two things I would like to do

It stops me from doing most of the things I would like to do

It stops me from doing everything I would like to do

\section{PART 4}

SECTION 1: The following questions pertain to your medication-taking behavior.

Please indicate either Yes or No for each question.

Do you ever forget to take your medicine?

Are you careless at times about taking your medicine?.

When you feel better do you sometimes stop taking your medicine?....

Sometimes if you feel worse when you take your medicine, do you stop taking it?

SECTION 2: Finally, we would like to know how your lung/respiratory problem affects you at work. The following questions are about the effect that your lung/respiratory problem has had on your work during the past year. Please respond only if you are currently employed.

1. During the past year, estimate the TOTAL NUMBER of DAYS you EXPERIENCED lung/respiratory problems (Maximum $=365$ days): Days

2. During the past year, estimate the TOTAL NUMBER OF DAYS you MISSED FROM WORK because you experienced lung/respiratory problems: Days

3. During the past year, estimate the TOTAL NUMBER OF DAYS you were ABLE TO ATTEND WORK even when you experienced lung/respiratory problems: Days

4. During a typical 8-hour workday, when you experienced lung/respiratory problems, estimate the TOTAL HOURS you were UNPRODUCTIVE because of your lung/respiratory condition (Maximum $=8$ hours): Hours

Please return the survey in the enclosed self-addressed business reply envelope. No postage is required. THANK YOU FOR YOUR TIME AND EFFORT! WE WISH YOU A LONG, HAPPY AND HEALTHY LIFE! 


\title{
CURRICULUM VITAE
}

\author{
ASHISH V. JOSHI \\ 20131 Heather Dr, West Windsor, NJ 08550 \\ Work: (609) 919-7948. Cell: (609) 933-3421. Fax: (609) 919-7704 \\ Email: ajsh@novonordisk.com
}

\section{AREAS OF RESEARCH INTEREST}

Decision Modeling and Pharmacoeconomic Evaluations, Claims Database Analysis, Pharmacoepidemiology, Health Policy and Quality of Life Research, Clinical Trial Design and Methodology

\section{ACADEMIC TRAINING AND QUALIFICATIONS}

2000 - 2004 Doctor of Philosophy (Ph.D.) in Pharmaceutical Systems and Policy, School of Pharmacy, West Virginia University, Morgantown, WV

Dissertation Title: Economic and Humanistic Impact of Medication Nonadherence in Patients with Asthma and Chronic Obstructive Pulmonary Disease

Dissertation Chairperson: Dr. S. Suresh Madhavan

1998 - 2000 Master of Science (MS) in Pharmaceutical Sciences, Dept. of Health Outcomes Research and Policy: University of Cincinnati, Cincinnati, $\mathrm{OH}$

Thesis Title: Health Care Utilization and Costs in Ohio Medicaid: Managed Care versus Feefor-Service

Thesis Chairperson(s): Dr. Theresa Shireman and Dr. Raymond Jang

1994 - 1998 Bachelor of Sciences (BS) in Pharmaceutical Sciences, Mumbai Educational Trust's Institute of Pharmacy, University of Bombay, India

\section{WORK EXPERIENCE}

Novo Nordisk Inc., Princeton, NJ

2004 - Current Manager, Health Economics and Market Access Strategy

Job Responsibilities

- Coordinate on reimbursement studies on product portfolio during product development and lifecycle

- Develop and implement reimbursement strategies for current as well as future indications

- Develop price list strategies with upper management 
- Advise medical and marketing departments on specific studies in relation to endpoints of clinical trials

- Develop, implement, and lead a clinical trial with respect to pharmacoeconomic strategy

- Incorporate business requirements/concerns of managed care and government into analytic tools and approaches, ie. preparation of AMCP dossiers and budget impact models

- Generate and analyze data to demonstrate cost-effectiveness of Novo Nordisk® products vs. competitor's products to support pricing and reimbursement negotiations in coordination with brand teams and managed care account executives

\section{Pfizer Inc., New York, NY}

2002 - Principal Investigator, Research Grant funded by Pfizer Inc., for project "Impact of Medication Nonadherence on Economic and Health-Related Quality of Life Outcomes in Patients with Asthma and Chronic Obstructive Pulmonary Disease.” Total Funding: \$20,000, Grant/Contract \# NY01002927803

2001 - Summer Intern, Pfizer Inc., Pfizer Global Pharmaceuticals, New York, NY. 2000 - 2004 Pfizer Outcomes Research Fellow, Pfizer Inc., New York, NY

Selected Pfizer Inc. Projects

-Involved in Pharmacoeconomic assessment of losses in workplace productivity due to 11 chronic conditions

- Involved in designing productivity questionnaire, designed methodology for assessing dollar value of productivity losses

- Conducted productivity impact analyses using MS Access and SPSS for various employers across the United States

-Coronary heart disease risk assessment projects using screening dataset

- Assigning cardiovascular disease risk to patients based on the prevalence of risk factors according to the Framingham algorithm

- Involved in aggregating data, conceptualizing research design, conducting data analyses

- Presenting findings at national meetings and manuscript writing -Costs calculations for Markov modeling for Spiriva ${ }^{\circledR}$

-Development of clinical pathways, screening protocols

\section{Ohio Medicaid}

1999 - 2000 Research Assistant, University of Cincinnati, Cincinnati, OH

\section{Selected Ohio Medicaid Projects}

-Health-Related Quality-of-Life Assessment in solid organ transplant patients. Principal Investigator: Dr. Jill Martin, University of Cincinnati. Assisted in primary data collection -Examining the success of Managed Care implementation for Medicaid recipients in Ohio, University of Cincinnati, Cincinnati, $\mathrm{OH}$. 
- Outcomes included rates of / costs due to adverse events such as hospitalizations and ED visits, as well as utilization / costs of outpatient visits and prescription drug use

- Study was conducted to supplement the results obtained from the Consumer Assessment of Health Plans Survey (CAHPS)

-Involved in various Drug Utilization Review (DUR) studies, Responsibilities included maintaining the prescription claims database in MS ACCESS ${ }^{\circledR}$, carrying out statistical analyses using SAS ${ }^{\circledR}$, SPSS $^{\circledR}$ for WINDOWS.

\section{OTHER RESEARCH EXPERIENCE}

1999 - Project for multinational pharmaceutical company involving comparison of an investigational drug for atrial fibrillation with placebo using cost-effectiveness analysis, using Cost/QALY gained as an evaluation parameter

1998 - Involved in data collection for a project funded by Bristol-Myers Squibb Pharmaceuticals for an anti-hypertensive drug at the University of Rhode Island.

1997 - Summer Intern at Glaxo-Wellcome India Ltd, Bombay, India -Underwent practical training in all departments, including formulations, marketing and R\&D.

\section{TEACHING EXPERIENCE}

2004 - Teaching Assistant, Health Promotion and Disease Prevention Course, $3^{\text {rd }}$ year PharmD 2004 - Health-related Quality of Life Assessment, Health Outcomes and Policy Course, $3{ }^{\text {rd }}$ year PharmD

2001 - Cost-Effectiveness Analysis, Health Outcomes and Policy Course, $3^{\text {rd }}$ Year PharmD

\section{PROFESSIONAL DEVELOPMENT}

2003 - 2004 President, West Virginia University ISPOR Students' Chapter

Responsibilities: ISPOR Membership Outreach Committee Liaison, Fundraising Activities, Inviting Speakers, Conducting Journal Club

2001 - 2002 Vice-President, West Virginia University ISPOR Students' Chapter

2003 - Member, ISPOR Medication Compliance Special Interest Group

2002 - Member, ISPOR Managed Care-PBM Special Interest Group

2001 - Health Outcomes Research Seminar, Outcomes Research Group (Marketing

Department), Pfizer Inc, New York, NY

2001 - Risk Management Workshop, Pfizer Inc, New York, NY 
1999 - NCQA Accreditation for Managed Care Guidelines Seminar, Dr. Nick Dewan, Center for Quality Innovation and Research, University of Cincinnati, Cincinnati, OH.

1999 - Strategic Importance of Pharmacoeconomics Seminar, Dr. Samir K. Bhattacharyya, Pharmacoepidemiology and Pharmacovigilance Group, Proctor and Gamble Pharmaceuticals, Cincinnati, $\mathrm{OH}$.

\section{SELECTED PRESENTATIONS}

Joshi AV, Mathew P, Gore T. Cost-effectiveness of different treatment regimens consisting of NovoSeven ${ }^{\circledR}$ vs. FEIBA VH for the home treatment of minor-to-moderate bleeds in hemophilia patients with inhibitors in the United States: A Cost-of-Bleed model. Poster Presentation - The International Society on Thrombosis and Haemostasis $20^{\text {th }}$ Congress and $51^{\text {st }}$ Annual Meeting, August 6-12, Sydney, Australia.

Joshi AV, Gore T, Russell MW. Reduction in overdosage and cost savings associated with finer dosing increments through the use of Norditropin NordiFlex ${ }^{\circledR}$ in adults. Poster Presentation The Endocrine Society's $87^{\text {th }}$ Annual Meeting, June 4-7, San Diego, CA.

Joshi AV, Nicklasson L, Russell MW. Cost-utility of Norditropin ${ }^{\circledR}$ (rDNA Somatropin) in children with growth hormone deficiency. Poster Presentation - Tenth Annual ISPOR Meeting, May 15-18, Washington DC.

Joshi AV, Gore T, Russell MW. Cost savings associated with finer dosing increments through the use of Norditropin NordiFlex ${ }^{\circledR}$ in the United States. Poster Presentation - Tenth Annual ISPOR Meeting, May 15-18, Washington DC.

Joshi AV, Madhavan SS, Ambegaonkar A, Smith MJ, Scott V, Dedhia H. Economic burden of chronic obstructive pulmonary disease (COPD) in a state health insurance program. Poster Presentation - Ninth Annual ISPOR meeting, May 15-19, Crystal City, VA. Abstract published Value in Health 2004;7(3):257.

(May 2004)

Joshi AV, Madhavan SS, Ambegaonkar A, Smith MJ, Scott V, Dedhia H. Health-related quality of life in chronic respiratory disease. Poster Presentation - Ninth Annual ISPOR meeting, May 15-19, Crystal City, VA. Abstract published Value in Health 2004;7(3):257-8. (May 2004)

Joshi AV, Smith MJ. Rates of asthma prevalence and healthcare services utilization in a Medicaid population by demographic characteristics. Poster Presentation at the $19^{\text {th }}$ International Conference on Pharmacoepidemiology and the $1^{\text {st }}$ International Conference on Risk Management, August 21-24, Philadelphia, PA. Abstract published Pharmacoepidemiology and Drug Safety 2003;12:S39.

(August 2003) 
Joshi AV, Smith MJ. Rates of asthma-related medical and prescription resource utilization and costs in a Medicaid population. Poster presentation at the Eighth Annual ISPOR meeting, May 19-23, Crystal City, VA. Abstract published Value in Health 2003;6(3):370.

(May 2003)

Joshi AV, Day D, Lubowski T, Amonkar M, Ambegaonkar A. Impact of World Health Organization's (WHO) classification of Body Mass Index (BMI) on Systolic Blood Pressure and Lipids: An analysis of a multi-state screening project. Podium presentation at the $130^{\text {th }}$ Annual American Public Health Association meeting, Philadelphia, PA, November 2002. (November 2002)

Joshi AV, D'souza A, Madhavan SS. Differences in hospital length of stay, charges and mortality in congestive heart failure (CHF) patients. Poster Presentation at the Seventh Annual ISPOR International conference, Crystal City, VA, May 2002. Abstract published Value in Health 2002;5(3):167.

(May 2002)

Hassan M, Joshi AV, Madhavan SM, Amonkar MM. Impact of Obesity on Health-related Quality of Life (HRQoL): An analysis of Behavioral Risk Factor Surveillance System (BRFSS) Data. Poster Presentation at the Sixth International ISPOR conference, May 20-24, 2001, Crystal City, Virginia. Abstract Published Value in Health 2001;4(2):99.

(May 2001)

Joshi AV, Nau DP, Kalsekar IK. An Appraisal of Health-Related Quality of Life (HRQoL) Instruments for Use in patients infected with Human Immunodeficiency Virus (HIV) Disease. Poster Presentation at the Sixth International ISPOR conference, May 20-24, 2001, Crystal City, Virginia. Abstract Published Value in Health 2001;4(2):134.

(May 2001)

Shireman TI, Joshi AV. Prescription Utilization and Cost Differences among Three Therapeutic Classes between Medicaid FFS and Managed Care. Podium Presentation at the $148^{\text {th }}$ Annual American Pharmaceutical Association meeting, March 16-21, San Francisco, CA. Abstract published J Am Pharm Association 2001;41(2):327.

(March 2001)

Joshi AV, Shireman TI. The Impact of Insurance Coverage on Prescribing Patterns following an Outpatient visit in a Medicaid population. Poster Presentation at the $148^{\text {th }}$ Annual American Pharmaceutical Association meeting, March 16-21, San Francisco, CA. Abstract published J Am Pharm Association 2001;41(2):319.

(March 2001)

Joshi AV, Shireman TI, Jang R, Hanseman D. A Comparison of Health Care Costs in Ohio Medicaid: Managed Care versus Fee-for-Service. Podium presentation at the Midwestern Pharmacy Administration Conference, Toledo, $\mathrm{OH}$.

(July 2000) 


\section{PUBLICATIONS}

Joshi AV, Miller LA. Lack of Surgeon-Specific Information in Predicting Colon Cancer Outcomes (Letter). Annals of Internal Medicine 2004;140(12):1061-2.

Joshi AV, D'souza A, Madhavan SS. Differences in hospital length of stay, charges and mortality in congestive heart failure patients. $C H F$ 2004;10(2):76-84.

Hassan M, Joshi AV, Madhavan SS, Amonkar MM. Impact of Obesity on Health-related Quality of Life: An analysis of Behavioral Risk Factor Surveillance System Data. International Journal of Obesity 2003;27:1227-1232.

Joshi AV, Day D, Lubowski T, Ambegaonkar A. Impact of World Health Organization's classification of Body Mass Index on Systolic Blood Pressure and Lipids: An analysis of a multi-state screening project. (Under Review)

\section{RELEVANT COURSEWORK}

Economics: Pharmacoeconomics, Health Economics, Pharmaceutical Economics and Policy, Microeconomic Theory, Economic Data Analysis, Cost Estimation and Budgetary Impact Analysis Methodology (ISPOR), Certificate in Pharmaceutical Pricing Methodology (ISPOR) Statistics: Univariate, Multivariate, Non-parametric, Structural Equation Modeling, SAS Programming

Epidemiology: Introduction to Epidemiology, Pharmacoepidemiology, Chronic Disease Epidemiology, Decision Modeling Techniques

Research Methods: Research Methods and Design, Survey Research Methods, Health Outcomes Research

Health Administration: Healthcare Financial Management, Project Analysis/Evaluation, Health Care Organizations, Legal Health Administration

Marketing: Socio-Behavioral Theory, Consumer Behavior

\section{COMPUTER SKILLS}

Statistical Software (SAS ${ }^{\circledR}$, SPSS $^{\circledR}$ for Windows), Database Packages (Microsoft Access), Decision Analysis by TREE AGE ${ }^{\circledR}$, MS Word, MS Excel, MS Powerpoint.

\section{ACADEMIC AWARDS, FELLOWSHIPS AND HONORS}

2005 - Novo Nordisk Exceeding Expectations Award

2004 - ISPOR Distinguished Service Award

2002 - Rho Chi Pharmacy Honor Society

2000 - 2004 Pfizer Health Outcomes Research Fellowship, WVU School of Pharmacy

1999 - 2000 Research Assistantship, University of Cincinnati, Cincinnati, OH

1999 - 2000 Ohio State Medicaid DUR Grant Scholarship 
1999 - 2000 University Graduate Scholarship, University of Cincinnati, Cincinnati, OH 1998 - Award for obtaining $1^{\text {st }}$ rank, Fourth Year Bachelor of Pharmacy Examinations, MET's Institute of Pharmacy, University of Bombay, India

\section{REVIEWER}

2005 - Contributed Research Abstracts, $10^{\text {th }}$ Annual ISPOR Meeting, Washington DC 2004 - American Journal of Managed Care 2004 - Social Science and Medicine 2003 - Judge, Podium Presentations, $8^{\text {th }}$ Annual ISPOR meeting, Arlington, VA $2002-149^{\text {th }}$ Annual American Pharmacists' Association meeting (Economic, Social and Administrative Sciences abstracts), Philadelphia, PA

\section{PROFESSIONAL AFFILIATIONS}

2005 - Professional Pricing Society (PPS)

2004 - Haemostasis Forum

2004 - National Hemophilia Foundation (NHF)

2002 - American Public Health Association (APHA)

2000 - Current: International Society for Pharmacoeconomics and Outcomes Research (ISPOR)

2000 - American Pharmacists Association (APhA)

1994 - Life-Member, Indian Pharmaceutical Association (IPA) 